\title{
Cell-based assays to assess the immunostimulatory capacity of poultry vaccines
}

A rational approach to animal-free vaccine testing

Robin H.G.A. van den Biggelaar 
(C) 202I Robin H.G.A. van den Biggelaar.

All rights reserved. Published papers were reprinted in this pubication under CC license. No parts of this thesis may be reproduced or transmitted in any form or by any means without prior permission of the author.

ISBN: 978-90-393-7377-4

The research described in this thesis was performed at the Division of Immunology, Department of Biomolecular Health Sciences, Utrecht University, the Netherlands.

The research described in chapters 2-6 was funded by the Innovative Medicines Initiative 2 Joint Undertaking under grant agreement No II5924 (VAC2VAC). This Joint Undertaking receives support from the European Union's Horizon 2020 research and innovation program and EFPIA. The contents of this article represent the authors' scientific conclusions and neither IMI nor the European Union, EFPIA, or any Associated Partners are responsible for any use that may be made of the information contained therein.

Layout: Robin H.G.A. van den Biggelaar

Printing: proefschrift-aio.nl

Printing of this thesis was financially supported by Infection \& Immunity Utrecht and by the Division of Immunology, Department of Biomolecular Health Sciences, Utrecht University. 


\section{Cell-based assays to assess the immunostimulatory capacity of poultry vaccines}

A rational approach to animal-free vaccine testing

\section{Cellulaire testen ter beoordeling van de immunostimulatoire capaciteit van pluimvee vaccines}

Een rationele aanpak voor het dierproefvrij testen van vaccines

(met een samenvatting in het Nederlands)

\section{Proefschrift}

ter verkrijging van de graad van doctor aan de Universiteit Utrecht op gezag van de rector magnificus, prof.dr. H.R.B.M. Kummeling, ingevolge het besluit van het college voor promoties in het openbaar te verdedigen op donderdag 27 mei 202 I des middags te I 2.15 uur

door

Robin Henricus Godefridus Antonius van den Biggelaar geboren op 5 juli 1993 te Geldrop 
Promotoren:

Prof. dr.W.van Eden

Prof. dr.V.P.M.G. Rutten

Copromotor:

Dr. C.A. Jansen 



\section{Table of contents}

Chapter I General introduction

Chapter 2 Nitric oxide production and Fc receptor-mediated phagocytosis as functional readouts of macrophage activity upon stimuluation with inactivated poultry vaccines in vitro

Chapter 3 Macrophage activation assays to evaluate the immunostimulatory capacity of Avibacterium paragallinarum in a multivalent poultry vaccine

Chapter 4 In vitro chicken bone marrow-derived dendritic cells comprise subsets at different states of maturation

Chapter 5 Proteomic analysis of chicken bone marrowderived dendritic cells in response to an inactivated IBV + NDV poultry vaccine

Chapter 6 Assessment of antigen-specific T cell responses in chickens after vaccination with an inactivated vaccine for infectious bronchitis virus and Newcastle disease virus 
Chapter 7 Overcoming scientific barriers in the transition from in vivo to animal-free batch-release testing of human and veterinary vaccines

Chapter 8 Summarizing discussion

Appendix Nederlandse samenvatting

P 190

Dankwoord/Acknowledgements

P 194

Curriculum Vitae

P 197

List of publications

P 198 


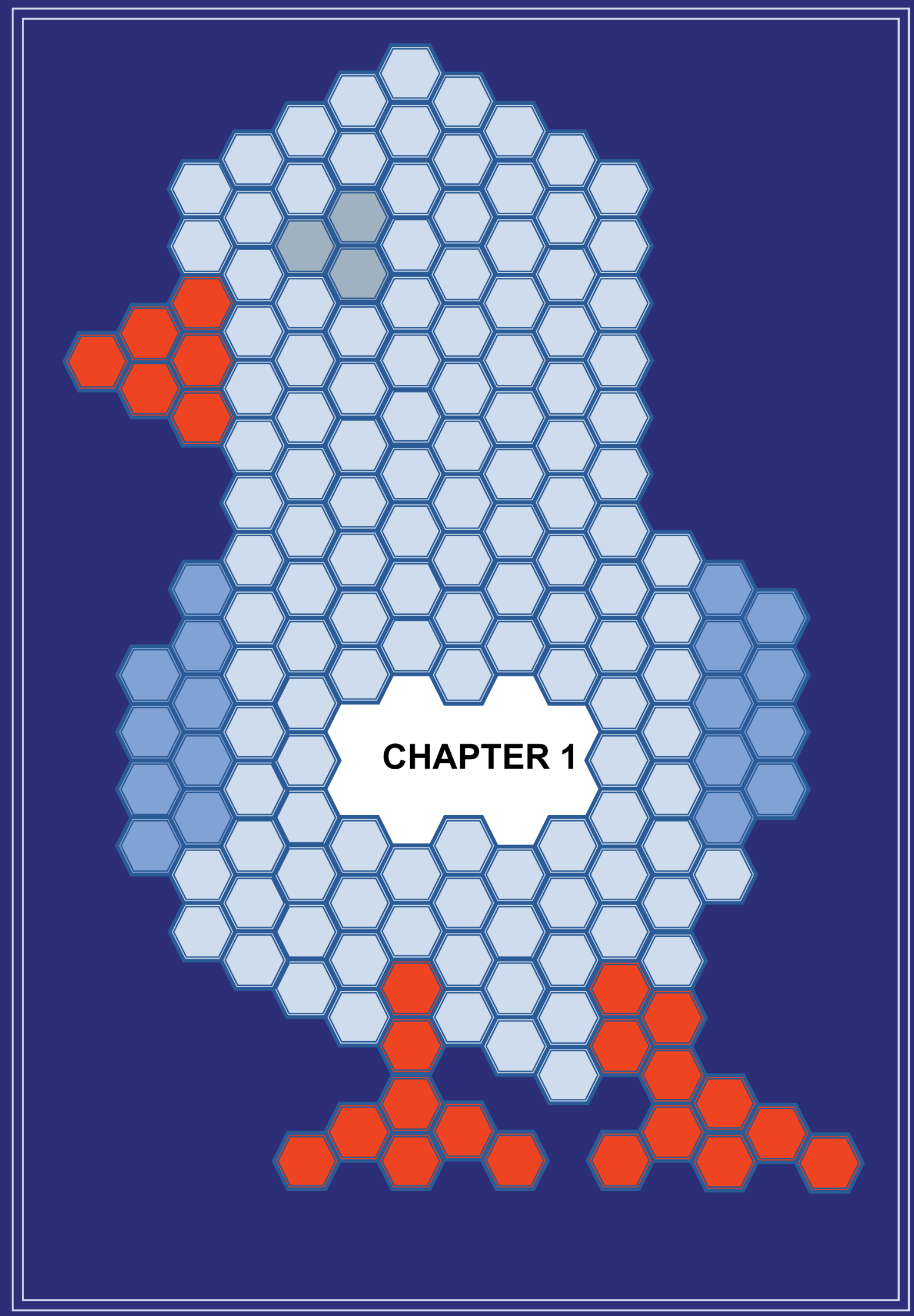


General introduction 


\section{General introduction}

Prevention of infectious diseases in humans and livestock relies on safe and efficacious vaccines. Vaccines are biologicals that, in many cases, comprise whole microorganisms or components thereof. Differences between vaccine batches may arise from small changes in culture conditions of these microorganisms prior to vaccine production, as well as from deviations in downstream production steps. Consequently, vaccine batches require routine quality control to test for compliance with the given specifications. For newer generation vaccines with a well-defined composition, e.g. recombinant subunit vaccines or DNA vaccines, quality may be assured through analytical assessment of the constituents [I-3]. In contrast, live attenuated and inactivated vaccines comprising whole organisms have a more complex composition, which complicates their assessment through analytical methods [4]. Batch quality assessment of live attenuated vaccine can be performed by culture and titration of the live pathogens present in the vaccines, but this is not possible for inactivated vaccines. The quality of inactivated vaccines is therefore often assessed through in vivo potency tests, in which the capacity of a vaccine batch to induce a protective immune response is evaluated.

In the poultry industry, animals are protected against infectious diseases by extensive vaccination schemes, which includes the use of many live attenuated and inactivated vaccines. Chickens receive the first live attenuated vaccines immediately after hatch, or even before hatch by in ovo vaccination, to provide early protection against infectious diseases like infectious bronchitis virus (IBV), Marek's disease virus (MDV) and Newcastle disease virus (NDV) [5]. Laying and breeding hens have a lifespan of more than a year during which the protective effect of vaccination may start to wane, which is why additional live attenuated or inactivated booster vaccines against e.g. IBV and NDV are administered before the laying period to enhance their immunity [6].

Inactivated poultry vaccines are still mostly tested for potency using in vivo vaccinationchallenge tests or serological tests, since in vitro alternatives are limitedly available. However, improved knowledge on the working mechanisms of vaccines has resulted in opportunities to develop new in vitro test methods, which could contribute to a reduction of animal use in vaccine quality testing by replacing currently used in vivo tests.

\section{Short history of vaccination}

In 1798, the first vaccination was performed by Edward Jenner, who vaccinated humans with cowpox virus, which causes only mild symptoms, to prevent severe infection with smallpox virus [7]. Smallpox was an infectious disease that caused high mortality worldwide until its eradication in 1977 as a result of a global vaccination campaign [8]. The word vaccination is named after the cowpox vaccine (Variolae vaccinae means "smallpox of the cow") and was introduced by Louis Pasteur to use for all practices that induce protective immunity against diseases in honor of Jenner [9].

In 1879, Louis Pasteur and co-workers created the first lab-grown live attenuated vaccine by using old bacterial cultures of Pasteurella multocida that had lost their virulence [9]. This vaccine could be used to protect chickens against fowl cholera, caused by virulent P. multocida bacteria. Pasteur used similar procedures to make an anthrax vaccine for use 
in sheep, cattle and horses, and a rabies vaccine, which was initially used in dogs and later in humans [10]. Many contemporary veterinary and human vaccines comprise viruses or bacteria that are attenuated by drying, heating or passage on tissue cultures, cell lines or embryonated chickens eggs [II].

In 1886, Daniel Salmon and Theobald Smith described the first inactivated vaccine, containing heat-killed Salmonella enterica [12]. Subsequently, heat-inactivated vaccines against typhoid, plague and cholera were successfully developed [13]. In contrast, heat-inactivation of diphtheria and tetanus did not result in effective and safe vaccines [14]. Emil von Behring and Kitasato Shibasaburo discovered that rabbits produced neutralizing antibodies upon injecting diphtheria toxin, the molecule responsible for the symptoms of diphtheria [15]. Subsequently, Smith and von Behring developed a safe and effective vaccine consisting of diphtheria toxin-antibody complexes $[14,15]$. The vaccine was further improved when Alexander Glenny and Henry Südmersen inactivated the toxin with formalin, resulting in a safer diphtheria toxoid vaccine [16]. In addition, they created precipitates of the toxoid vaccine by addition of acetic acid and potassium aluminium sulphate, resulting in stronger immune responses [17]. Additives that enhanced immune responsiveness were named adjuvants. Two aluminium salt adjuvants, including aluminium hydroxide and aluminium phosphate, are still used in human diphtheria and tetanus vaccines [18], as well as in many veterinary vaccines [19].

In the 1940s, water-in-oil emulsions were discovered as adjuvants, including Freund's complete adjuvant, containing mineral oil and inactivated Mycobacterium tuberculosis bacteria, and Freund's incomplete adjuvant, which lacks the bacteria [20-22]. Freund's adjuvants strongly stimulated antibody production, but were too reactogenic for use in humans [23]. Novartis' squalene-based MF59 emulsion adjuvant was licensed for flu vaccines in 1997 and the second adjuvant to be used in human vaccines [24]. Mineral oil adjuvants are still widely used in vaccines for veterinary use, including vaccines for poultry, cattle and fish [25].

\section{Vaccination in poultry}

Chickens are vaccinated mostly with live attenuated and inactivated vaccines, which are of low cost and suitable for mass vaccination. Live attenuated vaccines are administered en mass via mucosal routes through eye drops, drinking water or coarse sprays [26], and are used to prime (i.e., initiate) the immune response against infectious agents. Inactivated vaccines need to be administered to individual chickens by injection and are often formulated as multivalent vaccines to reduce time and costs of vaccination. Inactivated vaccines are given to laying and breeding hens just before the laying period for protection against infectious diseases during egg production and to stimulate the transfer of maternal antibodies to offspring $[27,28]$. To be effective, some inactivated vaccines require a prime-boost vaccination strategy, using a live attenuated vaccine followed by an inactivated vaccine against the same pathogen. Other inactivated vaccines provide protection upon single application, including vaccines for eggdrop syndrome virus (EDSV) [6], Salmonella [6], colibacillosis [29] and infectious coryza [30].

Poultry vaccines protect chickens from infectious diseases, but the type of immune responses (e.g. neutralizing antibodies or cellular responses) that lead to protection, the so-called 
correlates-of-protection, are often unknown. During the development phase, vaccines are mainly tested for their ability to induce protection. The induction serum antibody titers may be measured as well and is often presumed to correlate with protection. However, this presumption is not always based on supporting evidence from field studies [3I]. There are strong indications that $\mathrm{T}$ cells are important for protection against several avian pathogens [32].

Despite the availability of poultry vaccines for protection against many different avian infectious diseases, there is still need to further improve vaccine effectiveness, as demonstrated by evidence that IBV $[33,34]$ and NDV $[34,35]$ have become endemic worldwide and in major regions of the world, respectively. The protection of poultry against viral infections like IBV has been complicated by the emergence of new virus variants, which may also emerge from live attenuated vaccines that have reversed to virulence [35] by mutations or recombination events [36-38]. Recently, recombinant DNA [39,40] and viral vector vaccines [40,4I] have been developed in response to the unmet need for safer poultry vaccines and for vaccines that cross-protect against emerging pathogen variants. However, recombinant DNA, subunit and inactivated vaccines are less immunogenic compared to live attenuated vaccines and usually require adjuvants to be effective $[43,44]$.

\section{Overview of the immune system}

Cellular and humoral (i.e., part of body fluids) defense mechanisms of the immune system are divided into innate and adaptive components (Figure I). The innate component is present from birth, non-specific and immediately ready to combat a pathogen at first encounter. Innate immune cells are activated by pathogen-associated molecular patterns (PAMPs), e.g. bacterial lipopolysaccharides or viral nucleic acids, which are recognized through pattern recognition receptors (PRRs). Innate immune cells include innate lymphoid cells, monocytes, macrophages, natural killer cells, non-conventional T cells (e.g. $\gamma \delta \mathrm{T}$ cells), granulocytes and mast cells, which combat pathogens by direct killing or by secretion of pro-inflammatory cytokines. In addition innate immune cells include macrophages and dendritic cells, which also act as professional antigen-presenting cells (APCs). Professional APCs present peptides from intracellular pathogens or engulfed antigens on both major histocompatibility complexes (MHC) class I and II to T cells of the adaptive immune system (Figure 2). A third type of professional APC are B cells, which are part of the adaptive immune system. In contrast to professional APCs, other cells can only present antigens on MHC class I.

The adaptive immune response requires time to develop, is pathogen-specific and results in immunological memory. The latter refers to the phenomenon that upon second encounter with a pathogen, either due to previous infection or vaccination, the immune response will develop faster and stronger. The activation and differentiation of $\mathrm{T}$ cells requires three signals including antigen recognition, co-stimulation and cytokines (Figure 2). APCs express peptide-loaded MHC molecules that are recognized by $\mathrm{T}$ cells through $\mathrm{T}$ cell receptor (TCR) binding. In addition, APCs express co-stimulatory molecules in the presence of PAMPs or damage-associated molecular patterns (DAMPs) released from damaged or stressed tissues [45]. Finally, APCs, and other immune cells, release cytokines that influence the differentiation of T cells (Figure 3). 
T cells are divided into $\mathrm{CD}^{+}$or $\mathrm{CD} 8^{+} \mathrm{T}$ cells, which have different functions during an immune response. $\mathrm{CD}^{+} \mathrm{T}$ cells recognize peptides presented on $\mathrm{MHC}$ class II and differentiate into helper $T$ subtypes that each release effector cytokines to activate specific cells of the immune system (Table I). In contrast, $C D 8^{+} \mathrm{T}$ cells recognize peptides presented on MHC class I and differentiate into cytotoxic T cells, which kill infected and malignant host cells. Furthermore, both $\mathrm{CD}^{+}$and $\mathrm{CD} 8^{+} \mathrm{T}$ cells differentiate into memory $\mathrm{T}$ cells, which are reactivated upon a second encounter with the same pathogen and thus crucial for immunological memory. Some $\mathrm{CD}^{+} \mathrm{T}$ cells will differentiate into follicular helper $\mathrm{T}$ cells, which assist in the differentiation of $B$ cells that have recognized antigens through their $B$ cell receptor (BCR) into antibody-secreting plasma cells and memory B cells. Antibodies have diverse functions, including neutralization of pathogens, killing of bacteria through activation of the complement system, facilitating the uptake of pathogens by phagocytes (opsonization), and facilitating the depletion of infected or malignant host cells through activation of innate immune cells by antibody-dependent cellular cytotoxicity. The effector

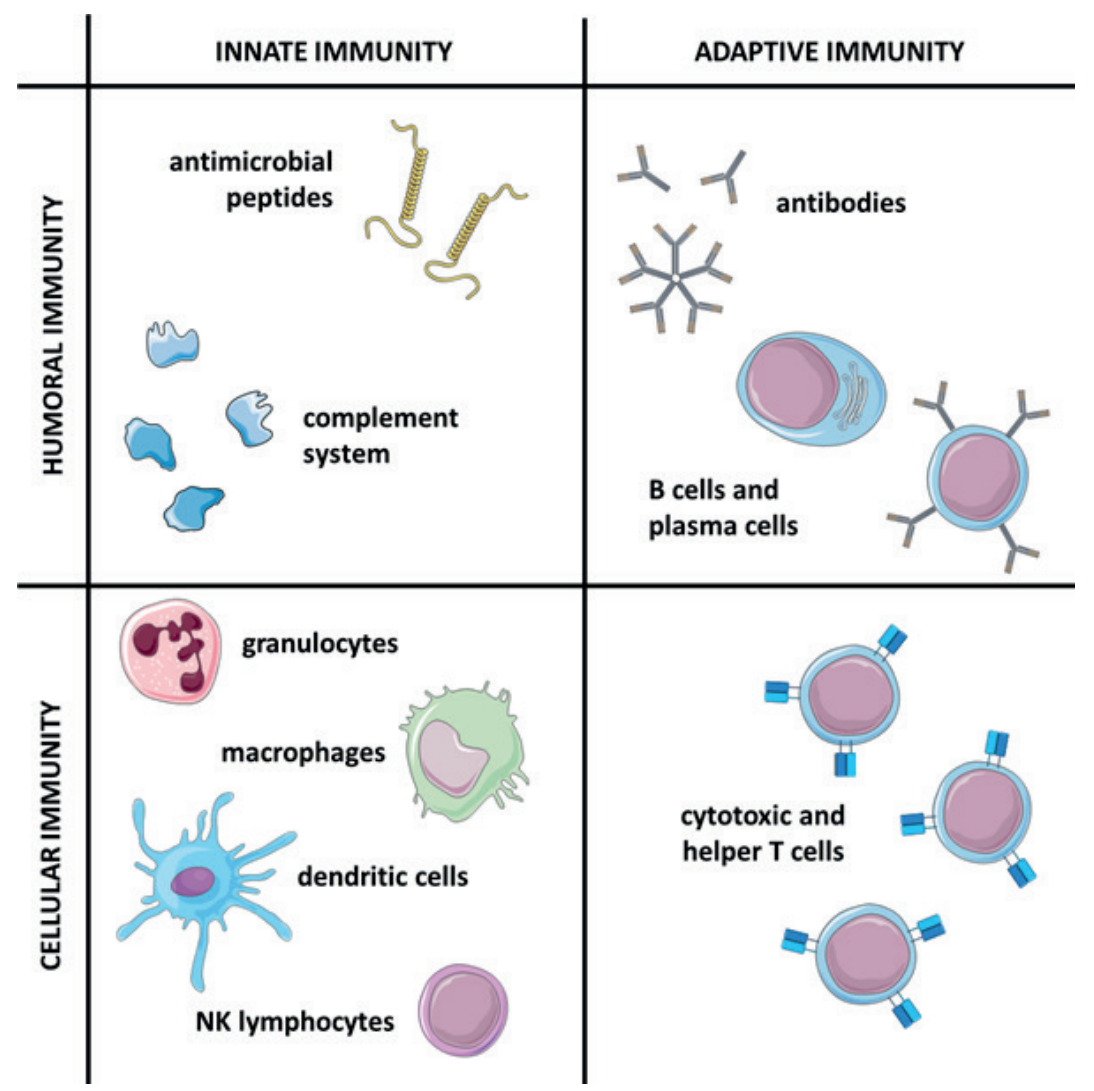

Figure I. Components of the immune system. The immune system is divided into innate and adaptive components that each consist of cellular and humoral components. B cells and plasma cells are shown as part of the humoral instead of the cellular component of the adaptive immune system due to their effector function in antibody secretion. The icons used in this figure are adaptations from icons retrieved from the Servier Medical Art collection, which are licensed under a Creative Commons Attribution 3.0 Unported License (https://creativecommons.org/licenses/by/3.0/). 


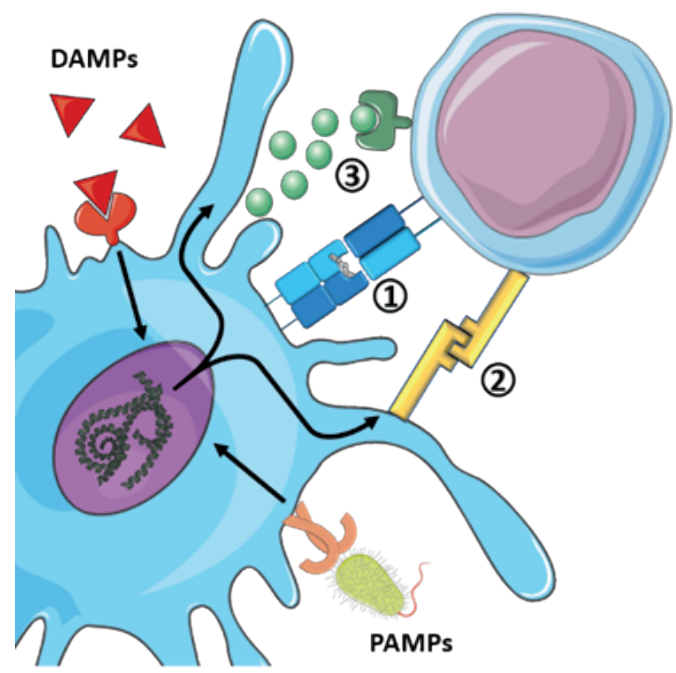

Figure 2. Three-signal model of T cell activation. APCs engulf and process antigens into peptides that can be presented on MHC molecules to T cells. T cells that recognize MHC-peptide complexes via their TCR will become activated, which is referred to as signal I. Furthermore, $T$ cells require two more signals for full activation including the binding of co-stimulatory receptors, providing signal 2 , and cytokines, providing signal 3. APCs provide these signals upon sensing that an antigen is 'dangerous' through recognition of PAMPs or DAMPs. Without signals 2 and 3 the T cell will become anergic and unable to further respond, which is a mechanism to prevent immune responses against 'safe' antigens. The combination of cytokines that are part of signal 3 determines $T$ cell differentiation into various $T$ helper subsets $\left(T_{H} \mathrm{I}, \mathrm{T}_{\mathrm{H}} 2\right.$, etc.). The icons used in this figure are adaptations from icons retrieved from the Servier Medical Art collection, which are licensed under a Creative Commons Attribution 3.0 Unported License (https://creativecommons.org/licenses/by/3.0/).

functions of antibodies depend on the various classes, which include immunoglobulin (Ig) $\mathrm{A}$, $\lg \mathrm{D}$, $\lg \mathrm{E}$, $\lg \mathrm{G}$ and $\lg M$ in mammals, and $\lg A$, $\lg \mathrm{g}$ and $\lg M$ in birds. Of these, naive $\mathrm{B}$ cells express $\lg D$ and $\lg M$ in mammals and $\lg M$ in birds. Antibody class switch to $\lg A$, $\lg E$ and $\lg G$ in mammals or $\lg A$ and $\lg Y$ in birds occurs when activated $B$ cells receive differentiation signals from follicular helper $T$ cells. The antibody most abundant in mucosal secretions is $\lg A$ in both mammals and birds. In serum, $\lg E$ and $\lg G$ antibody classes are present in mammals, whereas IgY is present in birds. Activated B cells further diversify in a process named somatic hypermutation, in which the antigen-binding regions of antibodies are enzymatically altered by gene mutations. Subsequently, follicular helper T cells are involved in a process called clonal selection, in which the $B$ cell clones with the highest affinity for antigens are selected for. The combination of somatic hypermutation and clonal selection results in antibodies of high affinity, which is referred to as affinity maturation.

Increased knowledge about the immune system has enabled a rational approach to vaccine design. A striking example is given by conjugate vaccines (e.g. Haemophilus influenzae type b), comprising bacterial polysaccharides linked to a carrier protein. Initially, the vaccine only contained polysaccharides, which did not result in effective humoral immune responses due to lack of proteins required for activation of $T$ cells and differentiation into follicular helper $T$ cells [46]. Another example is given by acellular pertussis vaccines, which lead to less effective $T_{H} 2 / T_{H} I 7$ responses as compared to effective $T_{H} I / T_{H} I 7$ responses induced by whole cell pertussis vaccines in mice and humans $[47,48]$. The induction of a $T_{H} I / T_{H} I 7$ response could be restored by substituting the aluminum salt adjuvant of acellular pertussis 


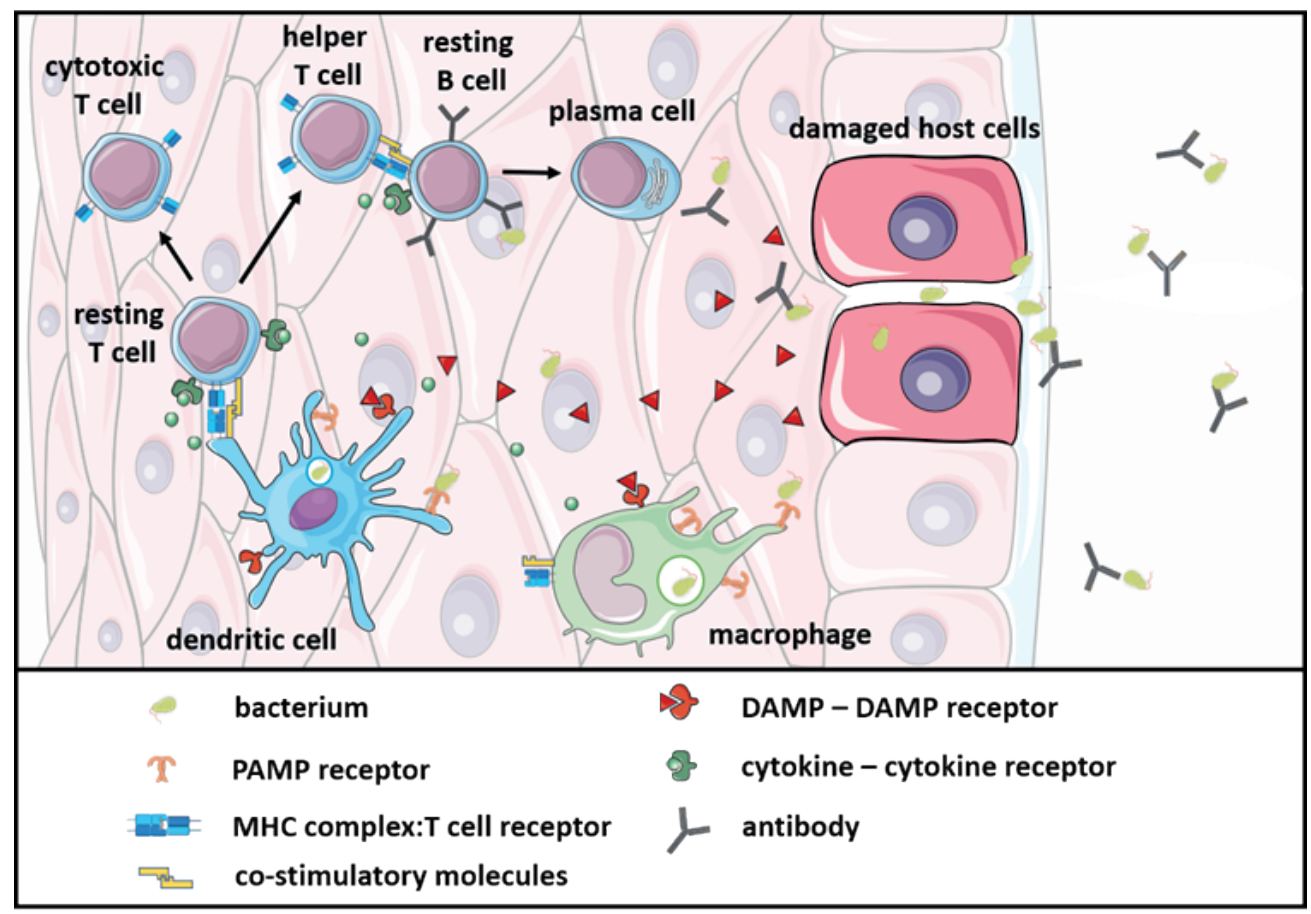

Figure 3. The immune system becomes active upon sensing pathogen-associated molecular patterns (PAMPs) or damage-associated molecular patterns (DAMPs). Antigen-presenting cells (APCs), including macrophages and dendritic cells, use pattern recognition receptors (PRRs) to sense the threat of e.g. an infection either directly through recognition of PAMPs or indirectly through recognition of DAMPs as a result of tissue injury. Simultaneously, APCs engulf and destroy pathogens, and process pathogenderived proteins into peptides for presentation on major histocompatibility (MHC) molecules. T cells become active when these possess $T$ cell receptors that are able to bind the MHC-peptide complexes presented on APCs. For full activation of T cells, co-stimulatory signals and cytokines are required, which are provided by APCs upon sensing PAMPs or DAMPs. Subsequently, some T cells will differentiate in helper $T$ cells that support the differentiation of antigen-specific $B$ cells into antibody-producing plasma cells, providing humoral immunity, whereas others will differentiate into cytotoxic T cells, providing cellular immunity. The icons used in this figure are adaptations from icons retrieved from the Servier Medical Art collection, which are licensed under a Creative Commons Attribution 3.0 Unported License (https://creativecommons.org/licenses/by/3.0/).

vaccines for $C_{P G}$ oligonucleotides a $T_{H} I / T_{H} I 7$, resulting in improved efficiency of vaccination [47]. Moreover, knowledge about the interaction between vaccines and the immune system allows the development of novel in vitro methods to assess the quality of vaccines as will be discussed below.

\section{Advancements in quality control of vaccines}

Vaccine manufacturers and regulatory authorities are responsible for assuring the quality and safety of vaccine batches in routine quality control, also after their initial approval. This is important since vaccines are often derived from living organisms that may show inherent variability resulting in differences between batches. 
Table I. Characteristics of main helper T subsets. This table gives an overview of the most distinct T helper subtypes. Abbreviations: $\mathrm{T}_{\text {EFF }}=$ effector $\mathrm{T}$ cells; $\mathrm{T}_{\mathrm{H}} \mathrm{I}$ (or 2, 17) = type I (or 2, 17) helper $\mathrm{T}$ cells; $T_{F H}=$ follicular helper $T$ cells and $T_{R E G}=$ regulatory $T$ cells.

\begin{tabular}{|c|c|c|c|c|c|c|}
\hline Type: & & $\begin{array}{l}\text { Master } \\
\text { regulator } \\
\text { (transcription } \\
\text { factor) }\end{array}$ & $\begin{array}{l}\text { Polarization } \\
\text { cytokines }\end{array}$ & $\begin{array}{l}\text { Signature } \\
\text { effector } \\
\text { cytokines }\end{array}$ & $\begin{array}{l}\text { Stimulates } \\
\text { (or inhibits) }\end{array}$ & Function \\
\hline \multirow{3}{*}{$\mathbf{T}_{\mathrm{EFF}}$} & $T_{H} I$ & T-bet & IL-I 2p70 & IFN- $\gamma$ & $\begin{array}{l}\text { macrophages, } \\
\text { cytotoxic T } \\
\text { cells, NK cells }\end{array}$ & $\begin{array}{l}\text { intracellular } \\
\text { pathogens }\end{array}$ \\
\hline & $\mathrm{T}_{\mathrm{H}} 2$ & GATA3 & IL-4 & $\begin{array}{l}\text { IL-4, IL-5, } \\
\text { IL-I } 3\end{array}$ & $\begin{array}{l}\text { eosinophils, } \\
\text { basophils, mast } \\
\text { cells }\end{array}$ & $\begin{array}{l}\text { extracellular } \\
\text { parasites }\end{array}$ \\
\hline & $T_{H} 17$ & $\mathrm{ROR} \gamma \mathrm{t}$ & $\begin{array}{l}\text { IL-6, IL-23, } \\
\text { TGF- } \beta\end{array}$ & $\begin{array}{l}\text { IL-I7A, IL- } \\
\text { I7F, IL-22 }\end{array}$ & $\begin{array}{l}\text { neutrophils (or } \\
\text { heterophils in } \\
\text { birds) }\end{array}$ & $\begin{array}{l}\text { extracellular } \\
\text { bacteria, fungi }\end{array}$ \\
\hline \multicolumn{2}{|l|}{$T_{F H}$} & $\mathrm{Bcl}-6$ & IL-6, IL-2I & IL-2I & B cells & $\begin{array}{l}\text { support } \\
\text { selection and } \\
\text { differentiation } \\
\text { of B cells }\end{array}$ \\
\hline $\mathbf{T}_{\mathrm{REG}}$ & & FoxP3 & IL-2,TGF- $\beta$ & $\begin{array}{l}\text { TGF- } \beta \text {, } \\
\text { IL-I0 }\end{array}$ & $\begin{array}{l}\text { T cells and } \\
\text { dendritic cells }\end{array}$ & $\begin{array}{l}\text { immune } \\
\text { tolerance and } \\
\text { resolution } \\
\text { of immune } \\
\text { responses }\end{array}$ \\
\hline
\end{tabular}

Traditionally, the quality of new batches of vaccine was assessed using in vivo vaccinationchallenge studies. The use of large numbers of animals in experiments that cause pain and distress leads to an ethical dilemma [49-5I]. Moreover, some animal models used are not reproducible or not representative for the natural pathophysiology of target pathogens [5I-55]. An example of the latter is the potency test for inactivated rabies vaccines, which requires intracerebral or intraperitoneal injection of rabies virus into mice, whereas the route of transmission is through saliva [5I]. Finally, the use of animal models is expensive, time consuming and may be a risk for personnel working with infected animals [5I].

The 3R guidelines by William Russell and Rex Burch describe how to enable 'Replacement', 'Reduction' and 'Refinement' in animal experiments [56]. Reduction can be accomplished by statistical estimation of the minimum number of animals required to perform vaccine quality tests [56]. Refinement has been accomplished by defining humane endpoints to avoid unnecessary suffering of animals [57]. Lethal endpoints can be substituted for early clinical signs that are predictive of death or for nonclinical endpoints such as the induction of antibody titers (Figure 4). Ideally, in vivo vaccine quality tests are replaced for non-animal in vitro alternatives. Recently, the European Pharmacopoeia Commission has described 'Removal' as a fourth ' $R$ ' when there is scientific evidence that an animal-based test is unnecessary and can thus be removed as a legal requirement for vaccine quality testing [58]. Directive 2010/63/EU on the protection of animals used for scientific purposes forbids the use of animal experiments when non-animal alternatives are available [59]. In line with this directive, the European Pharmacopoeia is continuously being updated to remove in vivo tests 
that are found to be unnecessary or to substitute tests for $3 R$ alternatives [58].

Advancements in in vitro culturing of live pathogens has resulted in batch potency tests of live attenuated vaccines that rely on viral or bacterial titration or bacterial counts rather than animal tests [60]. However, for inactivated and toxoid vaccines this is not possible, which is why these vaccines often require in vivo potency tests. Many in vivo vaccinationchallenge tests of inactivated and toxoid vaccines have been replaced by in vivo serological tests, which no longer require challenges with virulent pathogens [60]. Some in vivo potency tests of inactivated and toxoid vaccines have been replaced by in vitro antigen quantification methods, e.g. the tests for inactivated vaccines against hepatitis $A$ virus for use in humans [6I] and non-adjuvanted vaccines against Leptospira for use in dogs and cattle [62,63]. A major achievement for quality testing of poultry vaccines has been the implementation of an enzyme-lined immunosorbent assay (ELISA) based on antigen quantification as a potency test for inactivated vaccines against NDV [64].

One-to-one replacement of animal-based quality tests for established vaccines with in vitro alternatives can be realized when these are demonstrated to be a good correlateof-protection. Alternatively, replacement of animal-based vaccine batch-release tests can be accomplished through the consistency approach, which is based on the principle that quality is the consequence of consistent production of subsequent batches monitored by a good manufacturing practice (GMP) quality system [65]. In addition, final batch testing is performed by analysis of a limited number of parameters that together demonstrate consistent quality of vaccine batches [66]. Since 20I4, the consistency approach is promoted in the European Pharmacopoeia [67].

Box I. Relevant definitions for vaccine evaluation.

Vaccine efficacy is the maximum effect that can be expected and more specifically refers to the proportion of vaccinated individuals for which the desired effect has been accomplished under ideal conditions [ $3 \mathrm{I}]$. The desired effect may differ between products and may include for instance protection from infection, suffering or, in the case of livestock, reduced productivity.

Vaccine potency refers to the capacity (or strength) of a vaccine to exert the desired effect [4]. This may be solely related to infectivity (live vaccines) or antigen mass (killed or subunit vaccines) but can also be strengthened by the presence of immunostimulatory constituents (e.g. adjuvants or PAMPs).

Immunogenicity is the capacity of a vaccine (or other substance) to provoke an immune response against the vaccine antigens. In this way immunogenicity contributes to vaccine potency, provided that a beneficial type of immune response is elicited.

Vaccine batch-release potency tests assess the ability of vaccine batches to exert the desired effect directly or indirectly by measuring immune responses (correlates-of-protection) or vaccine parameters that correlate with the desired effect.

The consistency approach for vaccine batch-release quality testing implies the use of a set of in vitro assays that demonstrate consistency between product profiles of a new batch and a reference batch of proven efficacy and safety [66]. Consistent batches are expected to be similar with regard to potency and safety, which would render in vivo batch-release testing unnecessary. 
For some vaccines, antigen quantification methods are sufficient to fully replace in vivo potency tests. For others, a combination of in vitro immunochemical, physicochemical and cell-based assays may be required to assess different batches for consistency (Figure 4). These assays can be developed based on the current knowledge about the mechanisms by which vaccines activate the immune system. The use of cell-based assays has been proposed to mimic the most important aspects of the immune response, e.g. the expression of costimulatory molecules and cytokines [68-72].

\section{Outline of this thesis}

Currently, most inactivated poultry vaccines are tested for potency using in vivo vaccination-challenge or serological tests, which account for a significant proportion of laboratory animal use. The aim of this thesis is to set up in vitro cell-based assays to assess the immunostimulatory capacity of inactivated poultry vaccines and to determine their applicability for routine potency testing, in view of the 3 Rs. Development of in vitro alternatives for inactivated poultry vaccines is complicated by the presence of adjuvants and the fact that most are multivalent to simultaneously induce protection against many infectious diseases. Furthermore, some pathogens like IBV are high variably and comprise different strains, which makes it more difficult to develop antigen quantification methods that cover vaccines against these different strains. Finally, most studies that have investigated the immune responses evoked by inactivated poultry vaccines have focused on serology, which is why a comprehensive understanding of the immunostimulatory properties of these vaccines is often lacking.

The first two chapters of this thesis focus on the immunostimulatory properties of inactivated poultry vaccines by investigating their effect on chicken macrophages. Macrophages have a

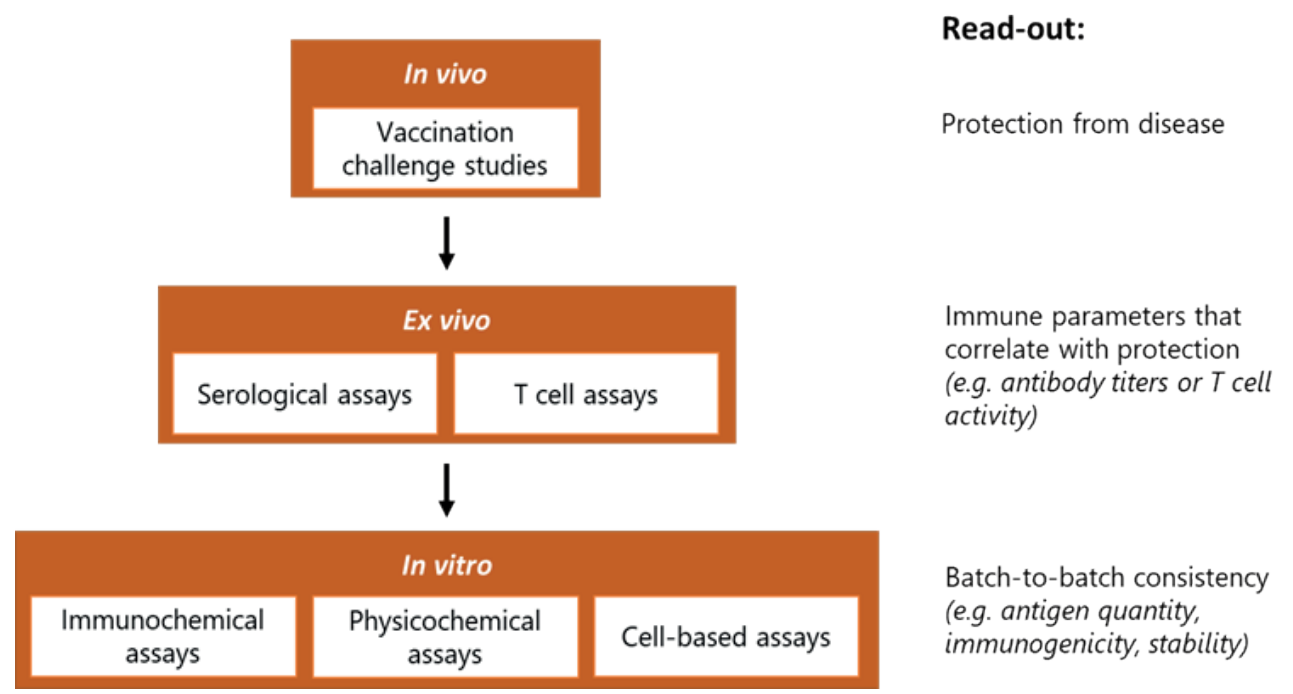

Figure 4. The transition from in vivo to in vitro vaccine batch quality testing. 
clear role in the early immune response [73,74] and express a broad range of PRRs [75], and are therefore able to sense immunostimulatory properties of vaccine constituents. The cell-based assays described involve the use of the macrophage-like cell line HDII, which has been used before to investigate the capacity of pathogens to stimulate innate immune cells [76-79]. In the study presented in Chapter 2, a phagocytosis assay with IgY-opsonized beads is used to investigate whether inactivated poultry vaccines against IBV, NDV and EDSV affect phagocytosis by macrophages. In Chapter 3, the use of nitric oxide production and cytokine gene expression are described to determine the immunostimulatory properties of an inactivated octavalent vaccine against IBV, NDV, EDSV and five strains of Avibacterium paragallinarum, the causative agents of infectious coryza characterized by an acute respiratory disease in chickens.

Due to their long lifespan, low variability, and availability in large numbers, immortalized cell lines may be more suitable for quality control settings than primary cells. However, immortalized cell lines may have reduced sensitivity [80] or impaired immune responsiveness [8I] as compared to their primary cell counterparts. Furthermore, dendritic cells, rather than macrophages, are considered to be most important for the induction of an immune response [69], and dendritic cell lines of chicken origin are not available. The study presented in Chapter $\mathbf{4}$ describes a thorough morphological, phenotypical and functional characterization of chicken bone marrow-derived dendritic cell cultures, using similar cell culture methods as previously described by others [82,83]. In Chapter 5, exposure of the bone marrow-derived dendritic cells to an inactivated poultry vaccine against IBV and NDV is described. The purpose of this study was to identify biomarkers of vaccineinduced immune responses by proteomic analysis. Furthermore, the mechanisms by which inactivated poultry vaccines activate dendritic cells were explored.

As previously mentioned, the quality of inactivated poultry vaccines is often determined in vaccination-challenge or serological tests. The induction of humoral immunity is known to be an important aspect of vaccination with inactivated poultry vaccines. In contrast, knowledge about the induction of T cell-mediated immunity is still limited, although there are studies that have demonstrated a role for cellular immunity in the protection against IBV [84] and NDV [85]. The study of Chapter 6 describes a T cell proliferation assay to evaluate cellular immunity in laying hens vaccinated with an inactivated vaccine against IBV and NDV and its potential as a quality test for this vaccine.

In Chapter 7, a review describes the difficulties that are being encountered during the transition from in vivo to in vitro batch testing of vaccines for both human and veterinary use, as well as possibilities to overcome these difficulties. Topics include the critical quality attributes of vaccines that require testing, the use of cell-based assays to mimic relevant aspects of in vivo vaccine-induced immune responses, the difficulties of adjuvanted and multivalent vaccines and how to overcome these, the use of altered batches to validate in vitro test methods when non-compliant vaccine batches are limitedly available, and how cooperation between different stakeholders is key to move the transition forward. Finally, the findings of this thesis, as well as the next steps required for the transition in vitro potency testing of inactivated poultry vaccines, are discussed in Chapter $\mathbf{8}$. 


\section{References}

I. EDQM. European Pharmacopoeia. 10.5th. Strasbourgh, France: European Department for the Quality of Medicines; 2020.

2. Shank-Retzlaff M, Wang F, Morley T, Anderson C, Hamm M, Brown M, et al. Correlation between mouse potency and in vitro relative potency for human papillomavirus Type 16 virus-like particles and Gardasil vaccine samples. Hum Vaccin (2005) I(5): I9I-197. doi:10.416I/hv. I.5.2126

3. Descamps J, Giffroy D, Remy E, Mortiaux F, Mareschal JC, Ponsar C, et al. A case study of development, validation, and acceptance of a non-animal method for assessing human vaccine potency. Procedia Vaccinol (20II) 5:184-19I. doi:I0.1016/j.provac.201I.10.018

4. Verch T, Trausch JJ, Shank-Retzlaff M. Principles of vaccine potency assays. Bioanalysis (2018) 10(3):I63-180. doi:10.4I55/bio-2017-0I76

5. Peebles ED. In ovo applications in poultry: A review. Poult Sci (2018) 97(7):2322-2338. doi: 10.3382/ ps/pey08I

6. Schijns VEJC, van de Zande S, Lupiani B, Reddy SM. Practical aspects of poultry vaccination In: Avian Immunology (2013):345-362. doi:10.1016/B978-0-12-396965-1.00020-0

7. Baxby D. Edward Jenner's inquiry; a bicentenary analysis. Vaccine (1999) 17(4):30I-307. doi:I0.1016/S0264-4I0X(98)00207-2

8. Global Commission (World Health Organization). The global eradication of smallpox : final report of the Global Commission for the Certification of Smallpox Eradication, Geneva, December 1979. 1980:122 p.

9. Pasteur L. Translation of an address on the germ theory. Lancet (I88I) II8(3024):27I-272. doi:I0.10I6/S0|40-6736(02)35739-8

10. Berche P. Louis Pasteur, from crystals of life to vaccination. Clin Microbiol Infect (2012) I8(SUPPL. 5): I-6. doi: I0. I I I /j. I469-069|.2012.03945.x

II. De Gregorio E, Rappuoli R. From empiricism to rational design: A personal perspective of the evolution of vaccine development. Nat Rev Immunol (2014) I4(7):505-5 I4. doi: I0.1038/nri3694

12. Salmon D, Smith T. On a new method of producing immunity from contagious diseases. Proc Biol Soc Washington (I886) 3:29-33. https://www.biodiversitylibrary.org/part/92I59.

13. Plotkin S. History of vaccination. Proc Natl Acad Sci U S A (20I4) III (34):I2283-I2287. doi: 10.1073/pnas. 1400472III

14. Kaufmann SHE. Remembering Emil von Behring: From tetanus treatment to antibody cooperation with phagocytes. MBio (2017) 8(I):e00II7-17. doi:10.1 I28/mBio.001 I7-17

15. Lombard M, Pastoret PP, Moulin AM. A brief history of vaccines and vaccination. Rev Sci Tech (2007) 26(I):29-48. doi:10.20506/rst.26.1.1724

16. Glenny AT, Südmersen HJ. Notes on the production of immunity to diphtheria toxin. J Hyg (Lond) (192I) 20(2): I76-220. doi:10.1017/S0022I72400033945

17. Glenny AT, Pope CG, Waddington H, Wallace U. Immunological notes. XVII-XXIV. J Pathol Bacteriol (I926) 29(I):3 I-40. doi:1 0.1002/path. 1700290106

18. Marrack P, McKee AS, Munks MW. Towards an understanding of the adjuvant action of aluminium. Nat Rev Immunol (2009) 9(4):287-293. doi:10.1038/nri25I0

19. Spickler AR, Roth JA. Adjuvants in veterinary vaccines: modes of action and adverse effects. J Vet Intern Med (2003) 17(3):273-28I. doi:10.1 I I I/j.1939-1676.2003.tb02448.x

20. Freund J, Mcdermott K. Sensitization to horse serum by means of adjuvants. Proc Soc Exp Biol Med (1942) 49(4):548-553. doi:10.3181/00379727-49-13625

21. Freund J, Casals J, Hosmer EP. Sensitization and antibody formation after injection of tubercle bacilli and paraffin oil. Proc Soc Exp Biol Med (1937) 37(3):509-513. doi:10.3181/00379727-379625

22. Freund J, Thomson KJ. Antibody formation and sensitization with the aid of adjuvants. J Immunol (1948) 60(3):383-398.

23. Di Pasquale A, Preiss S, Da Silva FT, Garçon N. Vaccine adjuvants: From 1920 to 2015 and beyond. Vaccines (20I5) 3(2):320-343. doi:10.3390/vaccines3020320 
24. O'Hagan DT, Ott GS, Nest G Van, Rappuoli R, Giudice G Del. The history of MF59 $₫$ adjuvant: a phoenix that arose from the ashes. Expert Rev Vaccines (2013) I2(I):13-30. doi:10.1586/ erv. 12.140

25. Aitken ID, Survashe BD. Observations on the serological and dermal responses of turkeys to a single subcutaneous inoculation of inactivated newcastle disease vaccine in mineral oil adjuvant. Avian Pathol (1974) 3(3):2II-222. doi: I0.1080/03079457409353833

26. Wilson HL, Gerdts V, Babiuk LA. Mucosal vaccine development for veterinary and aquatic diseases. In: Mucosal Vaccines: Innovation for Preventing Infectious Diseases (2019):8I I-829. doi:I0.1016/B978-0-12-81 1924-2.00048-I

27. Fahey KJ, Crooks JK, Fraser RA. Assessment by ELISA of passively acquired protection against infectious bursal disease virus in chickens. Aust Vet J (1987) 64(7):203-207. doi:I0.1III/j.175I-08I3.1987.tb|5I82.x

28. Darbyshire JH, Peters RW. Humoral antibody response and assessment of protection following primary vaccination of chicks with maternally derived antibody against avian infectious bronchitis virus. Res Vet Sci (I985) 38(I):I4-2I. doi:I0.10I6/s0034-5288(I8)3184I-I

29. Ghunaim H, Abu-Madi MA, Kariyawasam S. Advances in vaccination against avian pathogenic Escherichia coli respiratory disease: Potentials and limitations. Vet Microbiol (20I4) I72(I-2):I322. doi:10.1016/j.vetmic.2014.04.019

30. Gong $\mathrm{Y}$, Zhang $\mathrm{P}$, Wang $\mathrm{H}$, Zhu W, Sun H, He Y, et al. Safety and efficacy studies on trivalent inactivated vaccines against infectious coryza. Vet Immunol Immunopathol (20I4) I58(I-2):3-7. doi:10.1016/j.vetimm.2013.01.015

3I. Knight-Jones TJD, Edmond K, Gubbins S, Paton DJ. Veterinary and human vaccine evaluation methods. Proc Biol Sci (20I4) 28I(I784):20I32839. doi:I0.I098/rspb.20I3.2839

32. Dai M, Xu C, Chen W, Liao M. Progress on chicken T cell immunity to viruses. Cell Mol Life Sci (2019) 76(I4):2779-2788. doi:10.1007/s000 I8-0I9-03। I7-I

33. Bande F, Arshad SS, Omar AR, Hair-Bejo M, Mahmuda A, Nair V. Global distributions and strain diversity of avian infectious bronchitis virus: A review. Anim Heal Res Rev (2017) 18(I):70-83. doi: I0.10I7/SI4662523I7000044

34. World Bank, TAFS Forum. World Livestock Disease Atlas: A Quantitative Analysis of Global Animal Health Data (2006-2009).; 20II. https:/openknowledge.worldbank.org/ handle/I0986/27। I8.

35. Hicks JT, Dimitrov KM, Afonso CL, Ramey AM, Bahl J. Global phylodynamic analysis of avian paramyxovirus-I provides evidence of inter-host transmission and intercontinental spatial diffusion. BMC Evol Biol (2019) 19(I): I08. doi:I0.1 I86/s I2862-019-143I-2

36. Hopkins SR, Yoder HW. Reversion to virulence of chicken-passaged infectious bronchitis vaccine virus. Avian Dis (1986) 30(I):22I-223. doi:10.2307/I590639

37. Hou Y, Zhang L, Ren M, Han Z, Sun J, Zhao Y, et al. A highly pathogenic Gl- 19 lineage infectious bronchitis virus originated from multiple recombination events with broad tissue tropism. Virus Res (2020) 285:198002. doi:10.1016/j.virusres.2020.198002

38. Rohaim MA, EI Naggar RF, Abdelsabour MA, Mohamed MHA, El-Sabagh IM, Munir M. Evolutionary analysis of infectious bronchitis virus reveals marked genetic diversity and recombination events. Genes (Basel) (2020) II (6):605. doi: I 0.3390/genes I I 060605

39. Xu L, Ren M, Sheng J, Ma T, Han Z, Zhao Y, et al. Genetic and biological characteristics of four novel recombinant avian infectious bronchitis viruses isolated in China. Virus Res (2019) 263:8797. doi:10.1016/j.virusres.2019.01.007

40. Jazayeri SD, Poh CL. Recent advances in delivery of veterinary DNA vaccines against avian pathogens. Vet Res (2019) 50(I):78. doi:I0.1 186/s I3567-019-0698-z

4I. United States Department of Agriculture. Veterinary biological products licensees and permittees prepared. (2020). https://www.aphis.usda.gov/aphis/ourfocus/animalhealth/veterinary-biologics.

42. Bashir Bello M, Yusoff K, Ideris A, Hair-Bejo M, Hassan Jibril A, Peeters BPH, et al. Exploring the prospects of engineered Newcastle disease virus in modern vaccinology. Viruses (2020) |2(4):45I. doi: |0.3390/v |204045 | 
43. Wu P, Lu J, Feng L, Wu H, Zhang X, Mei M, et al. Antigen-sparing and enhanced efficacy of multivalent vaccines adjuvanted with immunopotentiators in chickens. Front Microbiol (2017) 8:927. doi:10.3389/fmicb.2017.00927

44. Gupta SK, Deb R, Dey S, Chellappa MM. Toll-like receptor-based adjuvants: enhancing the immune response to vaccines against infectious diseases of chicken. Expert Rev Vaccines (2014) 13(7):909-925. doi:10.1586/I4760584.20I4.920236

45. Matzinger P. Tolerance, danger, and the extended family. Annu Rev Immunol (1994) I2(1):991 1045. doi: I 0.I I 46/annurev.iy. I2.040194.0050I5

46. Rappuoli R, De Gregorio E, Costantino P. On the mechanisms of conjugate vaccines. Proc Natl Acad Sci U S A (2019) II6(1):14-16. doi:10.1073/pnas.1819612116

47. Ross PJ, Sutton CE, Higgins S, Allen AC, Walsh K, Misiak A, et al. Relative contribution of $T_{H} I$ and $\mathrm{T}_{H} 17$ cells in adaptive immunity to Bordetella pertussis: towards the rational design of an improved acellular pertussis vaccine. PLoS Pathog (2013) 9(4): I 003264. doi: I0.137I/journal.ppat. 1003264

48. Higgs R, Higgins SC, Ross PJ, Mills KHG. Immunity to the respiratory pathogen Bordetella pertussis. Mucosal Immunol (20I2) 5(5):485-500. doi:I0.1038/mi.20I2.54

49. Milne C, Terao E, Buchheit KH. EDQM's 3 Rs activities in the field of quality control of vaccines. ALTEX Proceedings (2012) I2(I):65-69.

50. Walker A, Srinivas GB. Opportunities and strategies to further reduce animal use for Leptospira vaccine potency testing. Biologicals (20I3) 4 I (5):332-337. doi:I0.10I6/j.biologicals.20I3.06.006

5I. Schiffelers MJ, Blaauboer B, Bakker W, Hendriksen C. Replacing the NIH test for rabies vaccine potency testing: A synopsis of drivers and barriers. Biologicals (2014) 42(4):205-2 I7. doi: I0. I0 I6/j. biologicals.2014.04.00I

52. Servat A, kempff S, Labadie A, Schereffer J-L, Boué F, Cliquet F. In vivo potency tests of rabies inactivated vaccines for veterinary use. A 2-year retrospective analysis of data according to the criteria of the European Pharmacopoeia. Pharmeur Bio Sci Notes (2008) 20:655-664.

53. Goris N, Merkelbach-Peters P, Diev VI, Verloo D, Zakharov VM, Kraft HP, et al. European Pharmacopoeia foot-and-mouth disease vaccine potency testing in cattle: Between test variability and its consequences. Vaccine (2007) 25(I7):3373-3379. doi:10.1016/j.vaccine.2006.12.049

54. Reed NE, Varney WC, Goddard RD, Wyeth PJ. The maintenance of challenge strains used in the potency test for canine leptospira vaccines. Biologicals (2000) 28(I):25-28. doi:10.1006/ biol.1999.0235

55. EDQM. General chapter 5.2.14 Substitution of in vivo methods by in vitro methods for the quality control of vaccines. In: European Pharmacopoeia. 10th ed. Strasbourgh, France: European Department for the Quality of Medicines; 2019.

56. Russell W, Burch R. The principles of humane experimental technique. Medical Journal of Australia (I960) I(I3):229. doi: I0.5694/j. I326-5377.1960.tb73I27.x

57. Hendriksen CFM. Humane endpoints in vaccine potency testing. Procedia Vaccinol (20II) 5:22I 226. doi:10.1016/j.provac.2011.10.022

58. Lang C, Kolaj-Robin O, Cirefice G, Taconet L, Pel E, Jouette S, et al. Replacement, Reduction, Refinement - Animal welfare progress in European Pharmacopoeia monographs: activities of the European Pharmacopoeia Commission from 2007 to 2017. Pharmeur Bio Sci Notes (2018) 2018:12-36.

59. The European Parliament and the Council of the European Union. DIRECTIVE 2010/63/EU on the protection of animals used for scientific purposes. Off J Eur Union (2010) 276:33-79.

60. Kumar S, Singh MP, Bharti VK, Pandey RP. Quality control of vaccines-A journey from classical approach to 3Rs. Microbiol Curr Res (20I8) 2(3):45-6I. doi:I0.4066/259I-8036.18-369.

61. Poirier B, Morgeaux S, Variot P, Fuchs F. In vitro potency assay for hepatitis A vaccines: development of a unique economical test. Biologicals (2000) 28(4):247-256. doi:I0.I006/biol.200 I.0264

62. Stokes W, Srinivas G, McFarland R, Kulpa-Eddy J, Casey W, Walker A, et al. Report on the international workshop on alternative methods for Leptospira vaccine potency testing: State of the science and the way forward. Biologicals. (20I3) 4I(5):279-294. doi:10.1016/j. biologicals.2013.06.013 
63. Klaasen HLBM, van der Veen M, Molkenboer MJCH, Bruderer U. Development of Leptospira in vitro potency assays: EU/industry experience and perspectives. Biologicals (20I3) 4 I (5):3 I5-322. doi:10.1016/j.biologicals.2013.06.012

64. Claassen I, Maas R, Daas A, Milne C. Feasibility study to evaluate the correlation between results of a candidate in vitro assay and established in vivo assays for potency determination of Newcastle disease vaccines. Pharmeuropa bio (2003) 2003(I):5I-66.

65. Stirling C. Consistency as tool to support in vitro batch potency testing in GMP production. Dev Biol (Basel) (20I2) I34:I I5-II8.

66. Hendriksen C, Arciniega JL, Bruckner L, Chevalier M, Coppens E, Descamps J, et al. The consistency approach for the quality control of vaccines. Biologicals (2008) 36(I):73-77. doi: 10.1016/j.biologicals.2007.05.002

67. De Mattia F, Hendriksen C, Buchheit KH, Chapsal JM, Halder M, Lambrigts D, et al. The vaccines consistency approach project: an EPAA initiative. Pharmeur Bio Sci Notes (2015) 2015:30-56.

68. Hoonakker ME, Verhagen LM, Hendriksen CFM, van Els CACM, Vandebriel RJ, Sloots A, et al. In vitro innate immune cell based models to assess whole cell Bordetella pertussis vaccine quality: $A$ proof of principle. Biologicals (2015) 43(2): 100-109. doi:I0.1016/j.biologicals.2014.12.002

69. Vandebriel R, Hoefnagel MMN. Dendritic cell-based in vitro assays for vaccine immunogenicity. Hum Vaccin Immunother (20I2) 8(9):I323-I325. doi:I0.4I6I/hv.2I350

70. Stoel M, Pool J, de Vries-Idema J, Zaaraoui-Boutahar F, Bijl M, Andeweg AC, et al. Innate responses induced by whole inactivated virus or subunit influenza vaccines in cultured dendritic cells correlate with immune responses in vivo. PLoS One (2015) I0(5):e0I25228. doi:10.137I/ journal.pone.0125228

7I. Tapia-Calle G, Stoel M, de Vries-Idema J, Huckriede A. Distinctive responses in an in vitro human dendritic cell-based system upon stimulation with different influenza vaccine formulations. Vaccines (2017) 5(3):2I. doi:10.3390/vaccines503002I

72. Hoefnagel MHN, Vermeulen JP, Scheper RJ, Vandebriel RJ. Response of MUTZ-3 dendritic cells to the different components of the Haemophilus influenzae type $B$ conjugate vaccine: Towards an in vitro assay for vaccine immunogenicity. Vaccine (20II) 29(32):5II4-5I2I. doi:I0.10I6/j. vaccine. 2011.05 .050

73. Grabowska J, Lopez-Venegas MA, Affandi AJ, Den Haan JMM. CDI69+ macrophages capture and dendritic cells instruct: The interplay of the gatekeeper and the general of the immune system. Front Immunol (2018) 9:2472. doi:I0.3389/fimmu.2018.02472

74. de Geus ED, Vervelde L. Regulation of macrophage and dendritic cell function by pathogens and through immunomodulation in the avian mucosa. Dev Comp Immunol (20I3) 4I(3):34I-35I. doi:10.1016/j.dci.2013.03.008

75. Iqbal M, Philbin VJ, Smith AL. Expression patterns of chicken Toll-like receptor mRNA in tissues, immune cell subsets and cell lines. Vet Immunol Immunopathol (2005) I04(I-2): I I7- 27. doi:I0.1016/J.VETIMM.2004.1I.003

76. Peng L, Matthijs MGR, Haagsman HP, Veldhuizen EJA. Avian pathogenic Escherichia coli-induced activation of chicken macrophage HDI I cells. Dev Comp Immunol (2018) 87:75-83. doi: I0. I0 I6/j. dci.2018.05.019

77. He H, Genovese KJ, Swaggerty CL, Nisbet DJ, Kogut MH. A comparative study on invasion, survival, modulation of oxidative burst, and nitric oxide responses of macrophages (HDII), and systemic infection in chickens by prevalent poultry Salmonella serovars. Foodborne Pathog Dis (20I2) 9(I2): I I04-III 0. doi:I0.1089/fpd.2012.1233

78. Crippen TL. The selective inhibition of nitric oxide production in the avian macrophage cell line HDI I. Vet Immunol Immunopathol (2006) I09(I-2):I27-I37. doi:I0.1016/j.vetimm.2005.08.024

79. Setta A, Barrow PA, Kaiser P, Jones MA. Immune dynamics following infection of avian macrophages and epithelial cells with typhoidal and non-typhoidal Salmonella enterica serovars; bacterial invasion and persistence, nitric oxide and oxygen production, differential host gene expression, NF- $\kappa B$ signalling and cell cytotoxicity. Vet Immunol Immunopathol (20I2) I46(34):2I2-224. doi:I0.10I6/J.VETIMM.20I2.03.008 
80. Studholme L, Sutherland J, Desai T, Hockley J, Care R, Nordgren IK, et al. Evaluation of the monocyte activation test for the safety testing of meningococcal $B$ vaccine Bexsero: A collaborative study. Vaccine (2019) 37(29):376I-3769. doi:I0.1016/j.vaccine.2018.05.073

81. Kwang DK, Choi SC, Noh YW, Jong WK, Paik SG, Yang Y, et al. Impaired responses of leukemic dendritic cells derived from a human myeloid cell line to LPS stimulation. Exp Mol Med (2006) 38(I):72-84. doi:10.1038/emm.2006.9

82. Wu Z, Kaiser P. Antigen presenting cells in a non-mammalian model system, the chicken. Immunobiology (20II) 2I6(I I): I I77-I I83. doi:I0.10I6/j.imbio.20II.05.0I2

83. de Geus ED, Tefsen B, van Haarlem DA, van Eden W, van Die I, Vervelde L. Glycans from avian influenza virus are recognized by chicken dendritic cells and are targets for the humoral immune response in chicken. Mol Immunol (2013) 56(4):452-462. doi:10.1016/j.molimm.2013.06.007

84. Seo SH, Pei J, Briles WE, Dzielawa J, Collisson EW. Adoptive transfer of infectious bronchitis virus primed alphabeta T cells bearing CD8 antigen protects chicks from acute infection. Virology (2000) 269(I): 183-189. doi: I0.1006/viro.2000.021।

85. Marino OC, Hanson RP. Cellular and humoral response of in ovo-bursectomized chickens to experimental challenge with velogenic Newcastle disease virus. Avian Dis (1987) 3I(2):293-30I. doi: $10.2307 / 1590875$ 
$\longrightarrow$ 


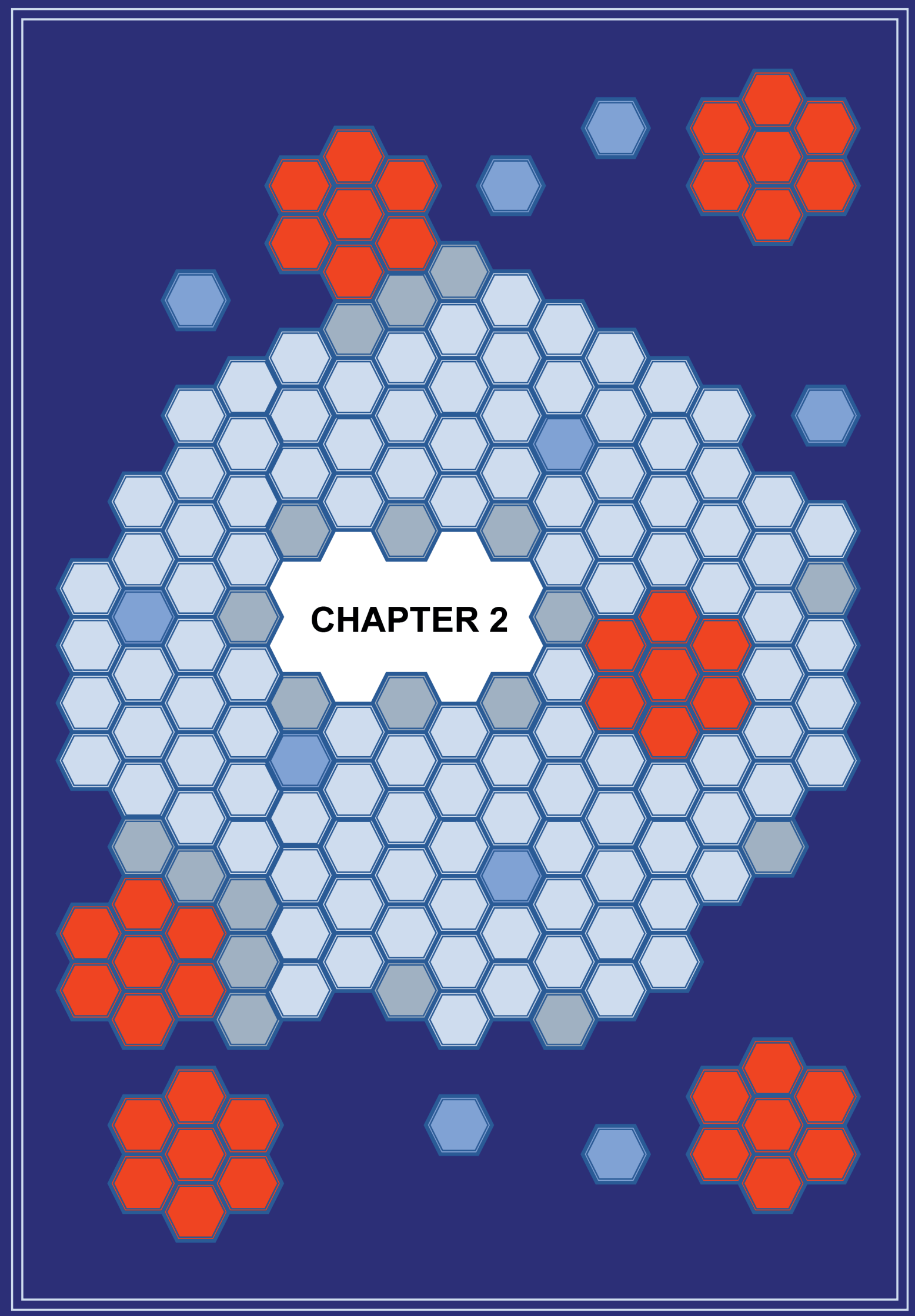


Nitric oxide production and Fc receptor-mediated phagocytosis as functional readouts of macrophage activity upon stimulation with inactivated poultry vaccines in vitro

Robin H.G.A. van den Biggelaar', Willem van Eden', Victor P.M.G. Rutten ${ }^{1,2}$ and Christine A. Jansen'

'Division of Infectious Diseases and Immunology, Department of Biomolecular Health Sciences, Faculty of Veterinary Medicine, Utrecht University, Utrecht, the Netherlands

${ }^{2}$ Department of Veterinary Tropical Diseases, Faculty of Veterinary Science, University of Pretoria, Pretoria, South Africa

Vaccines 2020, 8(2), 332

https://doi.org// 0.3390/vaccines8020332 


\section{Abstract}

Vaccine batches must pass routine quality control to confirm that their ability to induce protection against disease is consistent with batches of proven efficacy from development studies. For poultry vaccines, these tests are often performed in laboratory chickens by vaccination-challenge trials or serological assays. The aim of this study was to investigate innate immune responses against inactivated poultry vaccines and identify candidate immune parameters for in vitro quality tests as alternatives for animal-based quality tests. For this purpose, we set up assays to measure nitric oxide production and phagocytosis by the macrophage-like cell line HDII, upon stimulation with inactivated poultry vaccines for infectious bronchitis virus (IBV), Newcastle disease virus (NDV), and egg drop syndrome virus (EDSV). In both assays, macrophages became activated after stimulation with various toll-like receptor agonists. Inactivated poultry vaccines stimulated HDI I cells to produce nitric oxide due to the presence of mineral oil adjuvant. Moreover, inactivated poultry vaccines were found to enhance Fc receptor-mediated phagocytosis due to the presence of allantoic fluid in the vaccine antigen preparations. We showed that inactivated poultry vaccines stimulated nitric oxide production and Fc receptor-mediated phagocytosis by chicken macrophages. Similar to antigen quantification methods, the cell-based assays described here can be used for future assessment of vaccine batch-to-batch consistency. The ability of the assays to determine the immunopotentiating properties of inactivated poultry vaccines provides an additional step in the replacement of current in vivo batchrelease quality tests. 


\section{Introduction}

Infectious diseases are a major problem for the poultry industry and lead to economic losses. Therefore, vaccination is essential to prevent disease outbreaks and maintain flock health. Early in life, starting before, or soon after hatch, chickens are vaccinated frequently with live attenuated vaccines to induce protective immunity. Before the first laying period, layers receive several booster vaccinations to maintain this protective immunity during their egg production phase. These may include inactivated multivalent vaccines against a combination of pathogens, such as infectious bronchitis virus (IBV), Newcastle disease virus (NDV), egg drop syndrome virus (EDSV), and infectious bursal disease virus (IBDV), and are often formulated as water-in-oil (w/o) emulsions using mineral oil adjuvants to potentiate the immune response [I].

Since vaccines are complex biological products, each batch requires routine quality control (QC) testing to ensure that it meets the established requirements to induce protection against disease. Quality testing for the potency of inactivated poultry vaccines used to be performed with vaccination-challenge tests, which have nowadays largely been replaced with vaccination-serology tests $[2,3]$. However, for many infectious diseases of poultry, the correlates of protection are still unknown and serological assays may not always be the best method to monitor vaccine potency. Moreover, there is a growing global intent to further reduce the number of laboratory animals used for routine quality control of vaccine batches [4]. As a consequence of the implementation of the 3Rs (replacement, reduction, and refinement) in European law, animal testing in the EU is only allowed when no nonanimal alternatives are available [5]. The growing availability of physicochemical and cellbased alternatives has created opportunities to replace animal-based tests by methods that provide cost-effective and ethically attractive alternatives.

One strategy to ensure the quality of vaccines is the consistency approach, which implies that batch-to-batch variation of vaccines can be controlled by well-defined production processes and analysis of intermediate and final products by in vitro methods $[6,7,8]$. This approach requires a battery of methods to create a product profile that provides sufficient information to replace current animal-based tests. To assess antigen quantity, an important quality parameter of vaccines, a method like enzyme-linked immunosorbent assay (ELISA) can be used, which is in place for inactivated NDV vaccines $[3,9,10]$ Moreover, such methods have been developed for inactivated IBV and IBDV vaccines [ $10, \mathrm{II}]$, although they are not yet used for QC testing [3]. However, vaccine quality is usually not dependent on antigen quantity alone. Usually, additional vaccine components, like adjuvants, are required to stimulate the immune system, which is why antigen quantification methods should be complemented with other QC tests that assess the immunogenicity of these additives.

Pathogen-associated molecular patterns (PAMPs), like bacterial cell wall components or viral nucleic acids, activate innate immune cells via pattern recognition receptors (PRRs) $[12,13]$. Similarly, vaccine excipients, including adjuvants, can augment the immunogenicity of vaccines by activation of innate immune cells, either directly or by inflicting tissue damage, resulting in the release of endogenous danger signals, such as extracellular DNA, ATP, or heat-shock proteins, collectively called damage-associated molecular patterns (DAMPs) $[14,15]$. It is currently unknown whether inactivated viral poultry vaccines contain PAMPs 
or other constituents leading to DAMPs that augment immune responsiveness by the activation of innate immune cells. Immunopotentiating effects of vaccine constituents on innate immune cells can be addressed in vitro with cell-based assays. For poultry vaccines, a good candidate for such assays is the chicken macrophage-like cell line HDI I, which shows broad expression of PRRs, including toll-like receptors (TLRs) [16]. The HDII cell line has already been employed to explore the immune-activating or immunomodulatory properties of TLR agonists [17,18,19], liposomes [20], host defense peptides [2I], cytokines [22,23,24], bacteria $[17,21,25]$, and replicating viruses [26,27,28], using nitric oxide production as a functional readout of macrophage activation. Macrophages produce nitric oxide using the enzyme-inducible nitric oxide synthase (iNOS), which is expressed upon stimulation of PRRs and downstream activation of transcription factor nuclear factor kappa B (NF-אB) [I7]. In addition, the HDII macrophage-like cell line expresses a high-affinity Fc receptor for immunoglobulin $Y(\lg Y)$, the chicken Ig-like receptor ABI (CHIR-ABI) [29], and has been used before to study phagocytosis [30,3I]. In vivo, macrophages are strategically located at the entry sites of lymphoid tissues and are able to react to PAMPs and DAMPs in the blood lymph fluid [32,33]. Moreover, macrophages have been shown to augment immune responses after vaccination, due to their ability to produce nitric oxide and perform Fc receptor-mediated phagocytosis [34,35,36].

In this study, we stimulated the chicken macrophage-like cell line HDII with inactivated poultry vaccines and intermediary vaccine products, including antigenic fractions and mineral oil adjuvant, to study their effects on nitric oxide production and phagocytosis and to contribute to a better understanding of their effects on the chicken innate immune system. Moreover, we explored the potential of nitric oxide production and phagocytosis as biomarkers of vaccine-induced immune activation, for future use in in vitro vaccine $\mathrm{QC}$ tests.

\section{Materials and Methods}

\section{HDI I cell culture and stimulation}

The chicken macrophage-like cell line HDII [37], stored at $-140{ }^{\circ} \mathrm{C}$ in complete Roswell Park Memorial Institute (RPMI)- 1640 medium with 50\% Fetal Bovine Serum (FBS) and 10\% dimethyl sulfoxide (DMSO), was thawed and used after 3 to 20 passages. The cells were maintained in complete RPMI-I640 cell culture medium supplemented with GlutaMAX-I, phenol red, HEPES, I0\% fetal bovine serum (FBS), $200 \mathrm{U} / \mathrm{mL}$ penicillin, and $200 \mathrm{U} / \mathrm{mL}$ streptomycin (all Gibco, Life Technologies Limited, Paisley, UK) in Corning 75-cm2 cell culture flasks (Sigma-Aldrich, Saint Louis, MO, USA) at $37^{\circ} \mathrm{C}, 5 \% \mathrm{CO}_{2}$, and passaged twice weekly. For experiments, HDII cells were harvested from $75-\mathrm{cm} 2$ cell culture flasks when they were at $\sim 90 \%$ confluency using a $0.25 \%$ trypsin/EDTA solution supplemented with phenol red (Gibco, Life Technologies Limited, Paisley, UK). Subsequently, the cells were counted and resuspended at a concentration of 200,000 cells $/ \mathrm{mL}$. The cells were seeded at I mL/well complete RPMI medium in Corning Costar 24-well cell culture plates (SigmaAldrich, Saint Louis, MO, USA) and cultured overnight at $37^{\circ} \mathrm{C}$ and $5 \% \mathrm{CO}_{2}$.

After overnight incubation, the HDI I cells were exposed to various stimuli to assess their activation using either nitric oxide production or phagocytosis as a readout. Stimuli included 100-300 ng/mL lipopolysaccharides (LPS) from E. coli OI27:B8 (Sigma-Aldrich, Saint Louis, 
MO, USA) to target TLR4, 100-500 ng/mL CpG oligonucleotides (ODNs) 2006 to target TLR2I, $10 \mu \mathrm{g} / \mathrm{mL}$ resiquimod (R848) to target TLR7, $10 \mathrm{ng} / \mathrm{mL}$ Pam3CSK4 to target the TLR2/I heterodimer, and $5 \mu \mathrm{g} / \mathrm{mL}$ zymosan from S. cerevisiae (all InvivoGen, San Diego, CA, USA) to target the TLR2/6 heterodimer. In addition, HDI I cells were stimulated with established inactivated poultry vaccines and/or their antigenic fractions, which were kindly provided by three pharmaceutical companies that are part of the VAC2VAC consortium (http://www.vac2vac.eu/), hereafter referred to as companies A, B, and C. The inactivated poultry vaccines used in this study contained mineral oil adjuvants in w/o formulation and included inactivated monovalent IBV (company B), inactivated bivalent IBV + NDV (companies $A, B$, and $C$ ), and inactivated trivalent IBV + NDV + EDSV (company A) vaccines. The inactivated poultry vaccines from companies $A, B$, and $C$ were prepared in such a way that a single chicken vaccination dose corresponds to, respectively, $0.5,0.5$, and $0.3 \mathrm{~mL}$. The antigenic fractions, hereafter referred to as antigens, comprised whole inactivated IBV (companies A, B, and C) and NDV (company B), which were propagated on embryonated chicken eggs, harvested from the allantoic cavity, and inactivated using either formaldehyde or $\beta$-propiolactone. Allantoic fluid without virus (company A), mineral oil (company A), and an "empty vaccine" consisting of allantoic fluid without virus formulated with mineral oil (company B) were included as controls.

\section{Griess assay to measure nitric oxide production by HD I I cells}

Nitric oxide production by HDI I cells was measured by the Griess assay [38] $48 \mathrm{~h}$ after stimulation. First, $50 \mu \mathrm{L}$ of supernatants were harvested from triplicate wells and transferred to a 96-well flat-bottom plate (Corning B.V. Life Sciences, Amsterdam, The Netherlands) to measure the nitrite concentration. A 3.I3-200 $\mu \mathrm{M} \mathrm{NaNO}_{2}$ nitrite standard dilution series (Sigma-Aldrich, Merck, St. Louis, MO, USA) was included to generate a standard curve. Griess assay reagents were made by dissolving $\mathrm{N}$-(I-naphtyl)ethylenediamine at 3 $\mathrm{g} / \mathrm{L}$ and sulfanilamide at $10 \mathrm{~g} / \mathrm{L}$ (both from Sigma-Aldrich, Merck, St. Louis, MO, USA) in 2.5\% phosphoric acid (Supelco, Merck, St. Louis, MO, USA). The Griess reagents were mixed I:I and $50 \mu \mathrm{L}$ was added to the wells with cell culture supernatants and standards. The Griess reagents mixture turned purple upon reaction with nitrite ions in the cell culture supernatant. The optical density (OD) at $540 \mathrm{~nm}$ of each well was measured using a FLUOstar Omega microplate reader (BMG Labtech, Ortenberg, Germany) to determine the nitrite concentration of each sample according to the nitrite standard curve.

\section{Phagocytosis of IgY-Opsonized Beads by HDI I Cells}

\section{IgY-opsonization of fluorescent beads}

The phagocytosis assay was performed with chicken IgY-opsonized fluorescent beads, which were prepared by mixing I $\mu \mathrm{m}$ crimson carboxylate-modified FluoSpheres (Invitrogen, Life Technologies Europe BV, Bleiswijk, The Netherlands) at a final concentration of $7.2 \times 10^{9} /$ $\mathrm{mL}$ with an egg yolk IgY fraction (Agrisera $A B$, Vännäs, Sweden) at a final concentration of $14.4 \mathrm{mg} / \mathrm{mL}$ in a glass tube, followed by overnight mixing in an orbital rotator at $4{ }^{\circ} \mathrm{C}$. The next day, the beads were washed twice by adding $10 \mathrm{~mL}$ Dulbecco's phosphate-buffered saline without calcium and magnesium (DPBS ${ }^{--}$; Lonza, Basel, Switzerland) and centrifugated at $3000 \times \mathrm{g}$ for 20 min between washes. Finally, the beads were resuspended in DPBS ${ }^{-1-}$ at a concentration of $3.5 \times 10^{9}$ beads $/ \mathrm{mL}$. Coupling of IgY was confirmed by staining the beads with $0.5 \mu \mathrm{g} / \mathrm{mL}$ R-phycoerythrin (PE)-labeled mouse anti-chicken monoclonal antibodies (SouthernBiotech, Birmingham, AL, USA) in fluorescence-activated cell sorting (FACS) 
buffer containing DPBS ${ }^{-/}+0.5 \%$ bovine serum albumin and $0.005 \%$ sodium azide (both from Sigma-Aldrich, Saint Louis, MO, USA) and analysis using a CytoFLEX LX flow cytometer and 375-, 56I-, and 638-nm lasers (Beckman Coulter Inc., Brea, CA, USA) (Supplementary Materials Figure SI).

\section{Phagocytosis by HDI I cells}

Phagocytosis of IgY-opsonized beads was measured $24 \mathrm{~h}$ after stimulation of the HDII cells. First, three wells of a 24-well plate with HDII cells were harvested using DPBS 1- supplemented with $5 \mathrm{mM}$ UltraPure EDTA (Invitrogen, Life Technologies Europe BV, Bleiswijk, The Netherlands) to determine the cell counts per well, which ranged between 0.5 and $1.0 \times 10^{6}$ cells. IgY-opsonized beads were added at a I:I bead-to-cell ratio to the $\mathrm{HDI}$ I cells of the remaining wells followed by a 4 -h incubation at $37^{\circ} \mathrm{C}, 5 \% \mathrm{CO}_{2}$ to allow the cells to phagocytose the beads. Next, the cells were harvested using DPBS ${ }^{-1}$ supplemented with $5 \mathrm{mM}$ UltraPure EDTA and centrifuged at 400x $\mathrm{g}$ for $3 \mathrm{~min}$. The cells were transferred to 96-well V-bottom plates (Greiner Bio-One B.V., Alphen aan den Rijn, The Netherlands), washed in $\mathrm{DPBS}^{-/}$, and stained for cell viability in $50 \mu \mathrm{L} \mathrm{DPBS}^{--}$with 1:400 Zombie Aqua Fixable Viability Dye (BioLegend Inc., San Diego, CA, USA) for $20 \mathrm{~min}$ at $4{ }^{\circ} \mathrm{C}$. Subsequently, the cells were washed twice and fixed in $200 \mu \mathrm{L} \mathrm{DPBS}^{-/-}$with $2 \%$ paraformaldehyde (Alfa Aesar, Haverhill, MA, USA) for 10 min at room temperature (RT). Finally, the cells were washed once more in FACS buffer and resuspended in $200 \mu \mathrm{L}$ of FACS buffer. Up to 50,000 cells were analyzed using the CytoFLEX LX flow cytometer.

Data analysis was performed using Flowjo Software v. 10.6 (Flowjo LCC, Ashland, OR, USA) and Prism 8.4 (Graphpad Software Inc., San Diego, CA, USA). The viability of the cells was expressed as the percentage of HDI I cells negative for Zombie Aqua Fixable Viability Dye. Only samples with $\geq 100$ viable cells in the live gate (Figure $\mathbf{2 b}$ ) were included in the analysis of changes in bead uptake. The fluorescent content of HDI I cells measured using a 660/I0-nm bandpass filter after excitation with the 638-nm laser was directly proportional to the number of beads engulfed. Furthermore, the HDI I containing a single bead were visible as the first positive peak in a histogram showing the fluorescent intensity at $660 / 10$ $\mathrm{nm}$. From this, the average bead uptake by each sample was calculated by:

$$
\text { beads/cell }=\frac{M F I_{\text {total }}}{M F I_{\text {bead/cell }}}
$$

with $\mathrm{MFI}_{\text {total }}$ for the mean fluorescent intensity (MFI) at $660 / 10 \mathrm{~nm}$ of each sample $\mathrm{MFI}_{\text {I bead/cell }}$ for the MFI at $660 / 10 \mathrm{~nm}$ of cells containing I bead/cell (see also Figure Ia). Next, the fold change in bead uptake by HDII cells after stimulation was calculated by:

$$
\text { foldchange }=\frac{\text { beads/cell }}{\text { stimulated }}
$$

Flow cytometric side scatter to determine vaccine decomposition

An accumulation of vacuoles was observed in HDI I cells exposed to inactivated poultry vaccines or the empty vaccine control (without viral antigens), which was captured by light microscopy using an an EVOS FL microscope (AMG, Mill Creek, Washington, DC, USA). 
The vacuoles were considered to be vaccine-derived lipid droplets, since they were only observed in the presence of inactivated poultry vaccines containing emulsified mineral oil adjuvant. The accumulation of lipid droplets was considered as a surrogate marker for vaccine decomposition and quantified by measuring the average side scatter (SSC) of HDII cells by flow cytometry, in accordance with a previous study [39].

Involvement of the Fc receptor CHIR-AB I in IgY-opsonized bead uptake by HDI I cells HDI I cells stimulated for $24 \mathrm{~h}$ with different concentrations of LPS or inactivated IBV antigen (company $B$ ) were assessed for expression of $C H I R-A B I$ by subsequently staining the cells in $50 \mu \mathrm{L}$ of FACS buffer with I:20-diluted hybridoma supernatant containing mouse-antichicken CHIR-ABI (clone 8DI2, mouse IgG2b, gift from Thomas W. Göbel, LMU Munich, Munich, Germany) and $0.1 \mathrm{\mu g} / \mathrm{mL}$ allophycocyanin (APC)-labeled goat-anti-mouse IgG2a (SouthernBiotech, Birmingham, AL, USA) for $20 \mathrm{~min}$ at $4{ }^{\circ} \mathrm{C}$, with two washing steps in FACS buffer in between. Next, the cells were washed once in FACS buffer and once in DPBS $^{-1-}$ followed by staining in $50 \mu \mathrm{L}$ of DPBS ${ }^{-/}$with 1:400 Zombie Aqua Fixable Viability Dye (BioLegend Inc., San Diego, CA, USA) for 20 min at $4{ }^{\circ} \mathrm{C}$. Finally, the cells were washed once more in FACS buffer and resuspended in $200 \mu \mathrm{L}$ of FACS buffer for analysis using the CytoFLEX LX flow cytometer.

To determine the involvement of CHIR-ABI in the uptake of IgY-opsonized beads, different concentrations of mouse-anti-chicken CHIR-ABI were administered to HDII cells 10 min before addition of the beads to block interactions between CHIR-ABI and IgY-opsonized beads. Subsequent steps were according to the phagocytosis assay as described.

\section{Confocal microscopy to assess internalization of IgY-opsonized beads by HDI I cells}

HDII cells were prepared for confocal microscopy to confirm the internalization of IgY-opsonized beads. Ethanol-cleaned I2-mm glass coverslips (Waldemar Knittel Glasbearbeitungs $\mathrm{GmbH}$, Brunswick, Germany) were added to the 24-well cell culture plates before HDI I cells were seeded and subjected to the phagocytosis assay as described. After $4 \mathrm{~h}$ of incubation with $\mathrm{IgY}$-beads, the cells were washed twice with cold DPBS with calcium and magnesium $\left(\mathrm{DPBS}^{+++}\right.$; Lonza, Basel, Switzerland) before staining in DPBS ${ }^{+/+}$with $2 \mu \mathrm{g} /$ $\mathrm{mL}$ wheat germ agglutinin (WGA)-Alexa Fluor 488 (Invitrogen, Life Technologies Europe BV, Bleiswijk, The Netherlands) for $20 \mathrm{~min}$ at $4{ }^{\circ} \mathrm{C}$. Subsequently, the cells were washed thrice with cold $\mathrm{DPBS}^{+/+}$and fixed in $\mathrm{DPBS}^{+/+}$with $4 \%$ paraformaldehyde at RT for $30 \mathrm{~min}$. Next, the cells were washed three times with DPBS ${ }^{---}+10 \mathrm{mM}$ glycine (Merck Millipore, Burlington, MA, USA) to quench the remaining paraformaldehyde. The cells were washed once more in distilled water before the coverslips with HDII cells were mounted on Polysine microscope slides (Menzel Glazer GmbH \& Co KG, Braunschweig, Germany) using Fluorsave Reagent (Calbiochem, Merck Millipore, Burlington, MA, USA). The cells were captured, and bead internalization was analyzed using a TCS-SPE-II confocal microscope (Leica Microsystems B.V., Amsterdam, The Netherlands) and 488- and 635-nm diode lasers. Microscopic images were further processed using Fiji software [40].

\section{Statistical analysis}

Statistical analysis was performed using GraphPad Prism 8.4 software. When the assumptions of normally distributed data and residuals were met, a one-way ANOVA with Holm-Sidak's 
multiple comparisons test was used to test for statistically significant differences between stimulated and unstimulated control samples. When the assumptions of normality were not met, a non-parametric Kruskal-Wallis test with Dunn's multiple comparisons test was used instead. A $p$-value of $<0.05$ was considered statistically significant.

\section{Results}

\section{High concentrations of inactivated poultry vaccines induce nitric oxide production by HDI I Cells}

Production of nitric oxide by HDII cells stimulated with TLR agonists, used as positive controls, was determined by the Griess assay. Stimulation with LPS resulted in 106.1 \pm 2.2 $\mu \mathrm{M}, \mathrm{C}_{\mathrm{PG}}$ in I I5.2 $\pm 4.2 \mu \mathrm{M}$, and R848 in $84.4 \pm \mathrm{I} .4 \mu \mathrm{M}$ nitric oxide in the culture supernatant (Figure I a). Stimulation of HDI I cells with dose ranges of inactivated IBV and NDV antigens provided by different companies did not result in nitric oxide production (Figure Ib). In contrast, nitric oxide was produced at low quantities when HDII cells were stimulated with an inactivated monovalent IBV vaccine from company $B(4.8 \pm 0.5 \mu \mathrm{M})$ and inactivated bivalent IBV + NDV vaccines from companies A $(4.2 \pm 0.3 \mu \mathrm{M})$ and $B(4.9 \pm 0.5 \mu \mathrm{M})$ (Figure Ic). An "empty vaccine" without inactivated viral antigens also induced the production of low quantities of nitric oxide $(4.9 \pm 0.2 \mu \mathrm{M})$. All vaccines contained a mineral oil adjuvant and were formulated as water-in-oil emulsions. Nitric oxide production was not significantly different from unstimulated HDI I cells when non-emulsified mineral oil was added to the HDII cells (respictively $2.0 \pm 0.3 \mu \mathrm{M}$ and $2.6 \pm 0$.I $\mu \mathrm{M}$ ). Taken together, small amounts of nitric oxide were produced by HDII cells upon exposure to the inactivated poultry vaccines, which may be induced by the presence of emulsified mineral oil.

\section{Phagocytosis of IgY-opsonized beads by HDII Cells is enhanced upon stimulation with TLR agonists}

The ability of HDII cells to phagocytose IgY-opsonized beads after $24 \mathrm{~h}$ of stimulation with TLR agonists was assessed by a 4-h co-incubation (Figure $\mathbf{2 a}$ and Supplementary Materials Video SI). HDI I cells showed increased uptake of IgY-opsonized beads upon stimulation with LPS (2.18 \pm 0.05 -fold), CPG (1.99 \pm 0.12 -fold), R848 (1.66 \pm 0.04 -fold), Pam3CSK4 (I.87 \pm 0.08 -fold), and zymosan ( $1.59 \pm 0.07$-fold) compared to unstimulated cells (Figure 2b,c). The viability of HDI I remained unaffected by stimulation with zymosan and was only slightly affected by LPS, CpG, R848, or Pam3CSK4 (Supplementary Materials Figure S2).

\section{Allantoic fluid-containing inactivated IBV and NDV antigens enhance phagocytosis by HDI I Cells}

Next, the effects of inactivated IBV and NDV antigens on phagocytosis by HDI I cells were determined. IBV antigen from company B (maximum fold change $3.54 \pm 0.19$ at $10 \mu \mathrm{L} / \mathrm{mL}$ ) led to a higher induction of bead uptake, at a lower dose, than IBV antigens from companies A (maximum fold change $2.69 \pm 0.35$ at $18 \mu \mathrm{L} / \mathrm{mL}$ ) or $C$ (maximum fold change $2.81 \pm 0.36$ at $30 \mu \mathrm{L} / \mathrm{mL}$ ) (Figure 3a). Inactivated NDV antigens from company B (maximum fold change $3.8 \mathrm{I} \pm 0.29$ at $10 \mu \mathrm{L} / \mathrm{mL}$ ) also enhanced phagocytosis by HDII cells. Beyond the doses inducing a maximum bead uptake, increasing doses of IBV and NDV antigen led to a decrease in phagocytosis (Figure 3a), concurrent with decreased cell viability (Figure $\mathbf{3 b}$ ). 
IBV and NDV antigens both contained viruses that were whole inactivated after propagation in embryonated chicken eggs and harvest from the allantoic cavity. For this reason, allantoic fluid from non-inoculated eggs was tested for its ability to stimulate phagocytosis and found to enhance phagocytosis by $3.06 \pm 0.2 \mathrm{I}$-fold (Figure 3c). Similar to the IBV and NDV antigens, increasing doses of allantoic fluid also led to a decrease in cell viability (Figure $\mathbf{3 d}$ ).

(a)

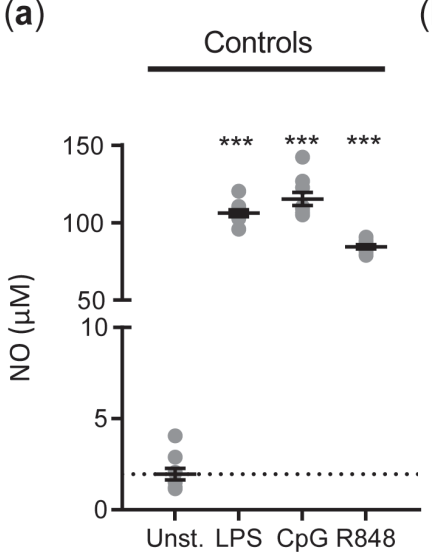

(b)

$\begin{array}{ccc}\text { Inactivated IBV antigen } & \text { Company B } & \begin{array}{c}\text { Inactivated } \\ \text { NDV antigen }\end{array} \\ \text { Company A } & \text { Company B }\end{array}$

(c)

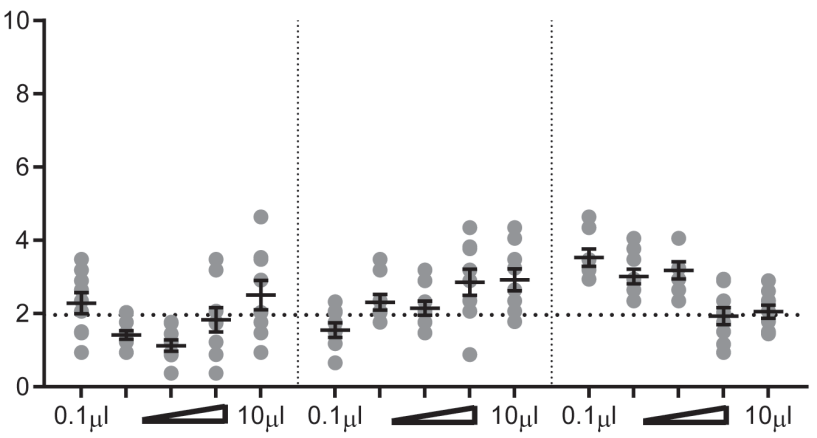
Inactivated IBV vaccine Inactivated Company B $\begin{array}{lll}\text { Company A Company B } & \text { Company B } & \text { Company A }\end{array}$

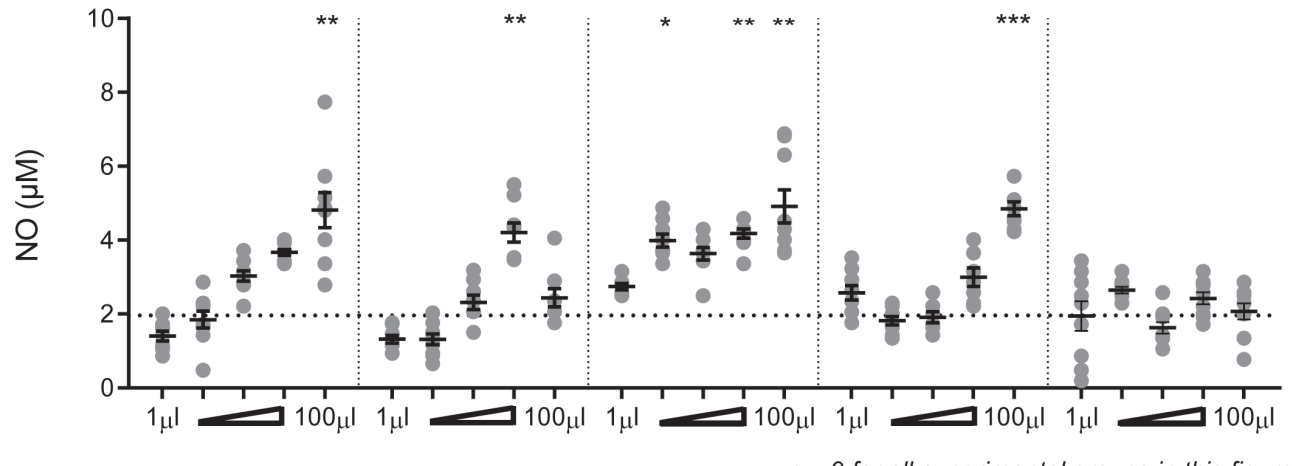
$n=9$ for all experimental groups in this figure

Figure I. Inactivated poultry vaccines induced nitric oxide production by HDII cells, whereas inactivated IBV and NDV antigens did not. (a) Nitric oxide production by HDII cells was assessed upon stimulation with TLR agonists, i.e., $100 \mathrm{ng} / \mathrm{mL}$ LPS, $100 \mathrm{ng} / \mathrm{mL}$ CpG, and $10 \mu \mathrm{g} / \mathrm{mL}$ R848. (b) In addition, HDII cells were exposed to inactivated IBV and NDV antigens (companies A and B) in doses ranging from $0.1-10 \mu \mathrm{L} / \mathrm{mL}$. (c) Finally, HDII cells were exposed to vaccines, an "empty vaccine" containing allantoic fluid without inactivated viruses, and mineral oil in doses ranging from $\mathrm{I}-100 \mu \mathrm{L} / \mathrm{mL}$. Three independent experiments were performed, and the experimental conditions of each independent experiment were tested in triplicate. Error bars represent the standard error of the mean (SEM). The experimental groups were tested for statistically significant increases in nitric oxide production as compared to unstimulated HDI I cells using a Kruskal-Wallis test and Dunn's multiple comparisons test. Statistical significance is indicated by $* p<0.05$, $* * p<0.0 \mathrm{I}$, and $* * * p<0.00 \mathrm{I}$. 


\section{Inactivated IBV and NDV vaccines can enhance phagocytosis by HD I I cells}

Five inactivated vaccines, all containing a mineral oil adjuvant in a w/o formulation, were used to stimulate HDII cells and included a monovalent IBV vaccine (company B), three bivalent IBV + NDV vaccines (companies $A, B$, and $C$ ), and a trivalent IBV + NDV + EDSV vaccine (company $A)$. Both bi- $(2.60 \pm 0.35$-fold) and trivalent (I.95 \pm 0.10 -fold) vaccines from company A led to a clear increase in the uptake of IgY-opsonized beads (Figure 4a). The bivalent vaccine from company $C$ resulted in maximal uptake at $10 \mu \mathrm{L} / \mathrm{mL}(1.49 \pm 0.06)$. The vaccines from company $B$ did not enhance phagocytosis. Vaccine doses beyond those inducing a maximum increase of phagocytosis resulted in decreased bead uptake, concurrent with decreased cell viability (Figure 4a and Supplementary Materials Figure S3). An increasing dose of bivalent vaccine from company $B$ also led to a decrease in cell viability without any enhancement of phagocytosis.

The mineral oil adjuvant of the vaccines acts as a slowly decomposing depot [4I], resulting in a gradual release of vaccine components, such as antigen, allantoic fluid, and mineral oil. We hypothesized that the decomposition rate of an emulsified vaccine affects its release of allantoic fluid and thus its effect on phagocytosis. Light microscopy showed the intracellular accumulation of vacuoles in HDI I cells exposed to the vaccines (Figure $\mathbf{4 b}$ ), suggesting that HDI I cells engulfed emulsified mineral oil released by the decomposing vaccines and stored this into lipid droplets. Because of this finding, we aimed to use flow cytometric SSC to quantify the accumulation of lipid droplets as a readout for vaccine decomposition. SSC can be used as a readout for engulfed particle content, as demonstrated by the high correlation between the SSC and the number of fluorescent IgY beads after the phagocytosis assay in unstimulated HDI I cells $\left(r^{2}=0.996\right)$ (Figure 4c). Next, SSC was found to become higher with increasing doses of the empty vaccine control from company $B$ until saturation was reached $\left(r^{2}=0.972\right)$ (Figure $\left.4 d\right)$, demonstrating that SSC could indeed be used to quantify vaccine decomposition. The SSC of HDI I cells upon exposure to the vaccines was analyzed to determine whether there was a relationship between the vaccine decomposition rate and bead uptake. For the bi- and trivalent vaccines from company A, bead uptake increased simultaneously with SSC, suggesting that the increase in bead uptake correlated with vaccine decomposition (Figure 4a,e). HDI I cells were saturated with lipid droplets after exposure to $30 \mu \mathrm{L} / \mathrm{mL}$ bivalent vaccine from company $A$, which is the dose at which phagocytosis was found to be maximally enhanced (Figure 4a,e). The SSC also increased with increasing concentrations of the mono- and bivalent vaccines from companies $B$ and $C$, but this did not result in similar changes in bead uptake. This indicates that differences between the vaccines in their capacity to induce phagocytosis cannot solely be explained by different decomposition rates.

\section{Fc receptor CHIR-ABI is responsible for the enhancement of IgY- opsonized bead uptake by HDI I cells upon exposure to inactivated IBV antigen}

Since we chose to use IgY-opsonized beads, we investigated whether the increase in phagocytosis was dependent on the high-affinity IgY Fc receptor CHIR-ABI. Its expression increased upon $24 \mathrm{~h}$ of stimulation with LPS (maximum $2.79 \pm 0.09$-fold change in $\mathrm{gMFI}$ ) or inactivated IBV antigen (maximum $2.23 \pm 0$. I9-fold change in gMFI) (Figure 5a,b). For IBV antigen, $C H I R-A B I$ expression reached its peak after stimulation with a dose of $10 \mu \mathrm{L} / \mathrm{mL}$ 
and decreased at higher doses, concurrent with the previously described cytotoxicity of the antigen (Figure 3b). Next, HDI I cells were incubated with a CHIR-ABI blocking antibody before performing the phagocytosis assay. The increased bead uptake upon exposure to an IBV antigen diminished with increasing concentrations of the blocking antibody (from $\mathrm{I} .83 \pm 0.23 \mathrm{beads} /$ cell to $0.32 \pm 0.04$ beads/cell) (Figure $\mathbf{5 d}$ ). In contrast, bead uptake in unstimulated or LPS-stimulated HDI I cells was less affected by CHIR-ABI blocking (Unst: from $0.67 \pm 0.05$ beads/cell to $0.50 \pm 0.04$ beads/cell; LPS: from I $.35 \pm 0.15$ beads/cell to 0.89 \pm 0.05 beads/cell).

\section{Discussion}

(a)

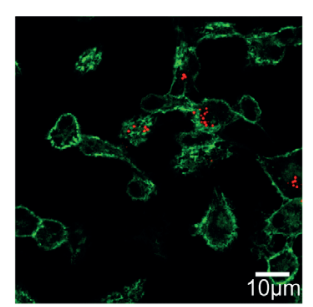

(b)

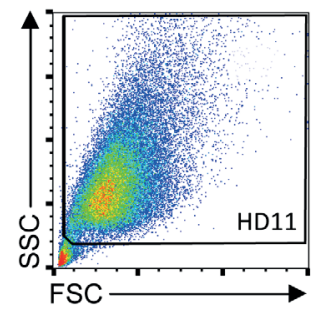

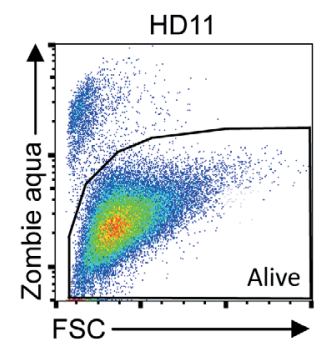

(c)

TLR agonists

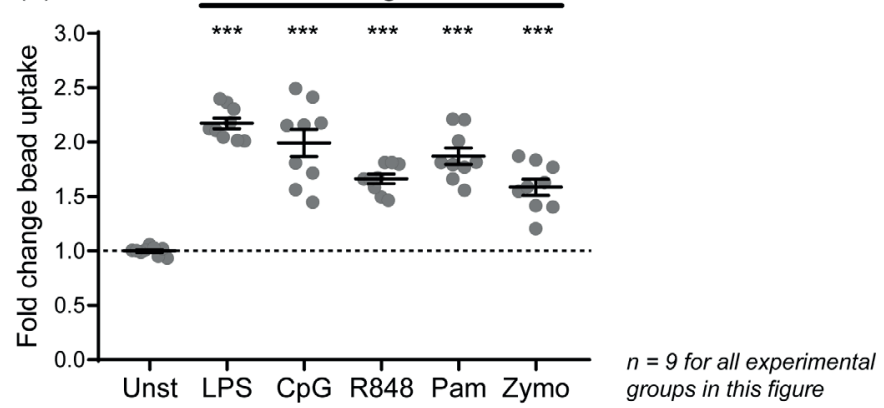

Figure 2. TLR agonists stimulated the uptake of IgY-opsonized beads by HDII cells. (a) Confocal microscopy confirmed the uptake of IgY-opsonized beads by HDII cells. The surface of unstimulated HDII cells was made visible by WGA-Alexa Fluor 488 shown in green and IgY-opsonized beads are shown in red. A corresponding video showing the 3-D model of this composition can be found in Supplementary Materials Video SI. (b) Bead uptake by HDII cells was quantified by flow cytometry. HDII cells were gated for their scatter profile (FSC/SSC) and viability (zombie aqua live/ dead staining). Moreover, HDI I cells with I bead/cell were gated to determine the fluorescence of a single bead, from which the average beads/cell for all HDI I cells could be calculated. (c) HDII cells were stimulated with $300 \mathrm{ng} / \mathrm{mL}$ LPS, $500 \mathrm{ng} / \mathrm{mL} C_{p}$, $10 \mu g / \mathrm{mL}$ R848, 10 ng/mL Pam3CSK4 (Pam), 5 $\mu \mathrm{g} / \mathrm{mL}$ zymosan (Zymo), or left unstimulated (Unst). The results are expressed as fold changes in bead uptake after stimulation in comparison to unstimulated controls. Three independent experiments were performed, and the experimental conditions of each independent experiment were tested in triplicate. Error bars represent the standard error of the mean (SEM). The experimental groups were tested for statistically significant differences in bead uptake between stimulated and unstimulated groups using a one-way ANOVA and Holm-Sidak's multiple comparisons test. Statistical significance is indicated by $* * * p<0.001$. 
(a)

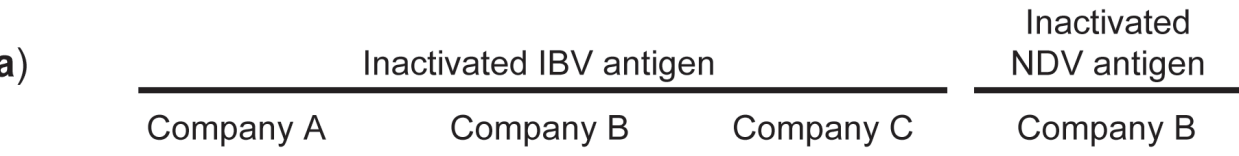
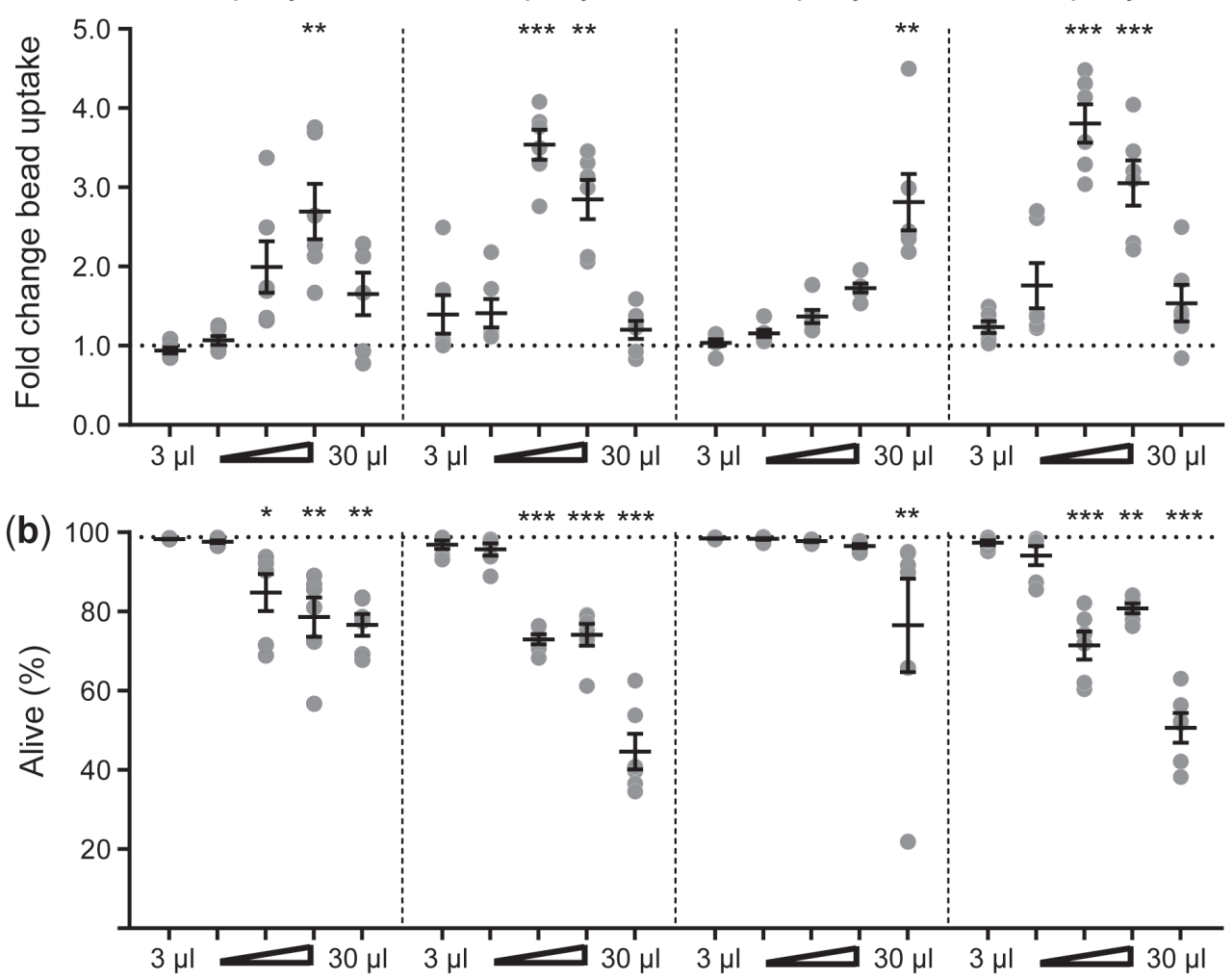

(c)

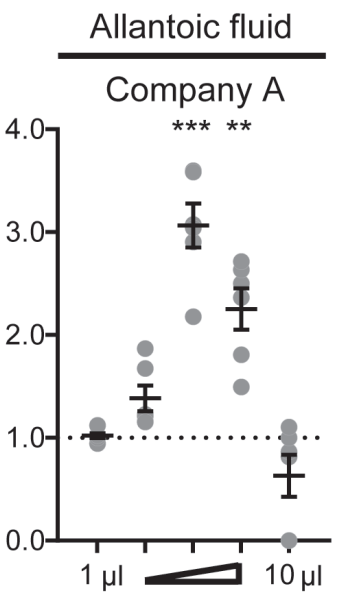

(d)

Allantoic fluid

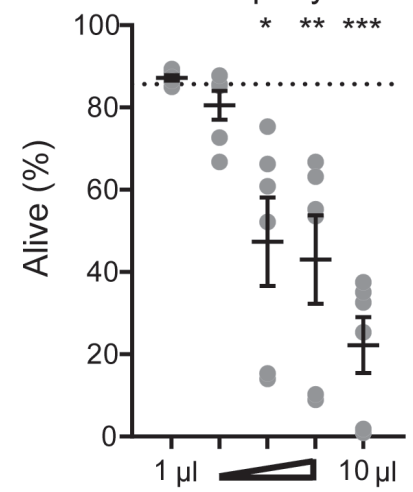

$n=6$ for all experimental groups of this figure

Figure 3. Phagocytosis of IgY-opsonized beads by HDII cells is increased upon exposure to inactivated IBV and NDV antigens. (a) Differences in bead uptake upon exposure to IBV and NDV antigens are expressed as fold changes compared to unstimulated controls. (b) The effects of IBV and NDV antigens on HDI I cell viability, as determined by Zombie Aqua Fixable Viability Dye, is expressed 
as the percentage of living cells (\% alive). Inactivated IBV antigens were provided by three different companies (A-C) and inactivated NDV antigen was provided by one company (B). In addition, the effects of allantoic fluid without virus (provided by company A) on HDII cell phagocytosis capacity (c) and cell viability (d) were determined. The $x$-axis shows the titrated doses at which IBV antigens, NDV antigens, or allantoic fluid without antigens were added, expressed as $\mu \mathrm{L}$ dose, added to I $\mathrm{mL}$ of cell culture medium. Three independent experiments were performed, and the experimental conditions of each independent experiment were tested in duplicate. Error bars represent the SEM. The experimental groups were tested for statistically significant differences in bead uptake and viability between stimulated and unstimulated groups using Kruskal-Wallis tests and Dunn's multiple comparisons tests. Statistical significance is indicated by $* p<0.05$, $* * p<0.01$, and $* * * p<0.00$ I.

In the present study, putative activating properties of inactivated poultry vaccines and their constituents on innate immune cells were assessed through measurement of nitric oxide production and phagocytosis by the chicken macrophage-like cell line HDII, as potential biomarkers for in vitro assessment of batch-to-batch consistency of vaccines. Small amounts of nitric oxide were produced in the presence of inactivated IBV and NDV vaccines or an empty vaccine without viral antigens. In contrast, nitric oxide was not produced in the presence of inactivated IBV or NDV antigens or non-emulsified mineral oil. These findings indicate that nitric oxide was produced by HDI I cells due to stimulation with the mineral oil adjuvant in its emulsified form.

The absence of nitric oxide production in the presence of viral antigens was unexpected, since HDII cells do express TLR7 [42] and TLR2I [43], PRRs for viral single-stranded RNA and double-stranded DNA, respectively. Moreover, TLR7 agonist R848 and TLR2I agonist CPG were able to induce high amounts of nitric oxide production. One hypothesis is that the nucleic acids from the inactivated viral antigens did not end up in the endosomes where these TLRs are present [42]. Alternatively, the recognition of the viral nucleic acids could be impaired by chemical modification, as a result of viral inactivation, or degradation. It has been shown for H5NI influenza vaccines that TLR7 is involved in the generation of an effective adaptive immune response and that TLR7 activation is severely reduced after inactivation of the antigen with formalin or $\beta$-propiolactone $[44,45]$.

The phagocytosis assays showed that both inactivated poultry vaccines and antigens enhanced the phagocytosis of IgY-opsonized beads by HDII cells. Moreover, allantoic fluid without antigens stimulated HDI I cells to enhance phagocytosis, similar to allantoic fluid containing inactivated IBV and NDV antigens. These results indicate that allantoic fluid, present in both the vaccines and antigen preparations, was responsible for the enhancement of phagocytosis. Allantois fluid contains a high concentration of uric acid, which may form monosodium urate crystals $[46,47]$. Previous studies have shown that uric acid is released from cells damaged by aluminum salt adjuvants and may form monosodium urate crystals that act as immunostimulatory DAMPs [47]. The stimulatory effects of allantoic fluid on phagocytosis may therefore be caused by the presence of uric acid precipitates. Vaccination studies in mice have shown that DAMPs stimulate antigen uptake by macrophages in vitro and enhance antibody titers in vivo $[48,49,50]$. Whether allantoic fluid stimulates phagocytosis and enhances antibody titers in chickens in vivo will be of interest for further investigation. 
(a)

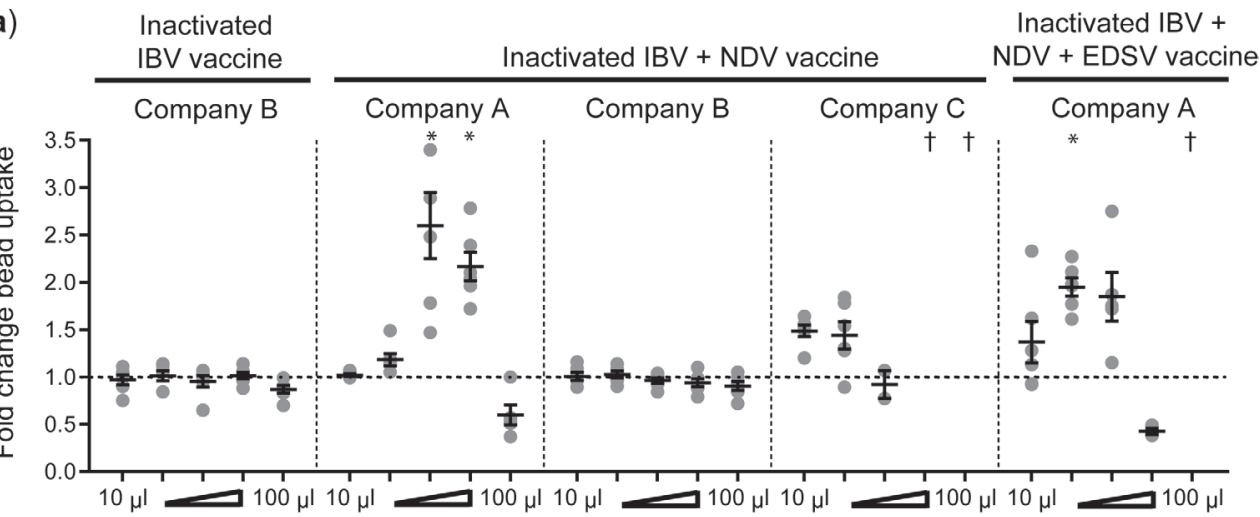

(b)

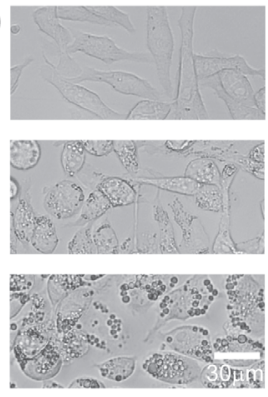

(c)

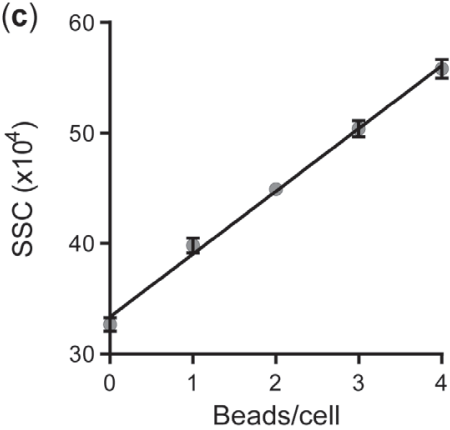

(d)

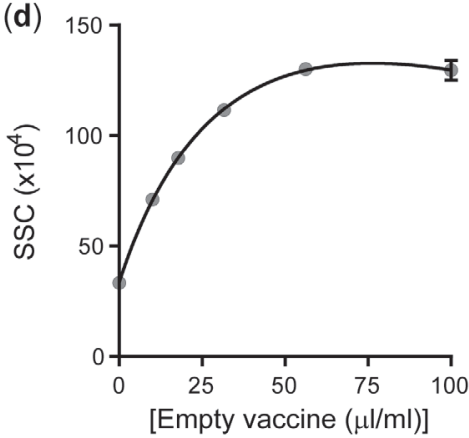

(e)
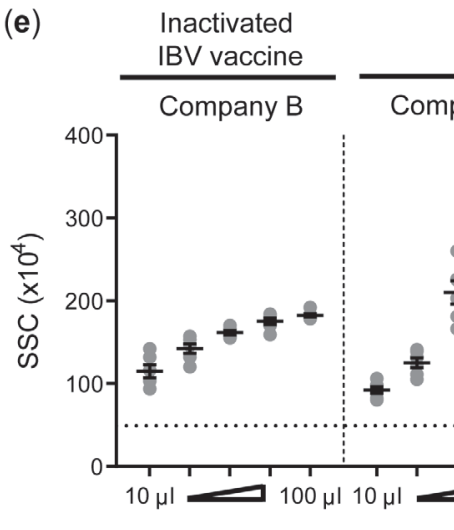

Inactivated IBV + NDV vaccine

Inactivated IBV + NDV + EDSV vaccine

Company A Company B Company C

Company $\mathrm{A}$
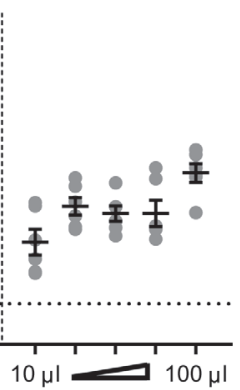

$n=6$ for all experimental groups of this figure

Figure 4. Phagocytosis capacity of HDII cells can be increased upon exposure to inactivated viral w/o vaccines. (a) The fold change in bead uptake by HDII cells upon stimulation with vaccines is compared to unstimulated controls. The $\mathrm{x}$-axis shows the graded doses at which the vaccines have been added, expressed as $\mu \mathrm{L}$ dose, added to $\mathrm{I} \mathrm{mL}$ of cell culture medium. $\dagger$ indicates that datapoints were missing because the threshold of $\geq 100$ viable cells was not reached. (b) Light microscopy photos show unstimulated HDII cells (top), HDII cells exposed to $10 \mu \mathrm{L} / \mathrm{mL}$ inactivated bivalent vaccine B (middle), and $100 \mu \mathrm{L} / \mathrm{mL}$ inactivated bivalent vaccine B (top). (c) A linear correlation curve shows the relationship between the average flow cytometric SSC and number of IgY-opsonized beads/cell for HDII cells containing 0-4 beads/cell. (d) A non-linear saturation curve shows the relationship between the average SSC and different doses of empty vaccine (without viral antigens) from 
company B. (e) The flow cytometric SSC of HDII cells is shown for graded doses of the different vaccines. Three independent experiments were performed, and the experimental conditions of each independent experiment were tested in duplicate. Error bars represent the SEM. The experimental groups were tested for statistically significant differences in bead uptake and SSC between stimulated and unstimulated groups using Kruskal-Wallis tests and Dunn's multiple comparisons tests. Statistical significance is indicated by $* p<0.05$. For $(\mathbf{e})$, all data was found to be statistically different from the unstimulated sample with $p<0.001$.

Stimulation with inactivated IBV antigens from different sources induced different outcomes of the phagocytosis assay, both in terms of the dose at which the highest bead uptake was observed and the maximal bead uptake, whereas no differences were observed between IBV and NDV antigens from the same source. Similarly, vaccines from different courses resulted in different outcomes. However, this does not affect the feasibility of assessing vaccine batch quality under the consistency approach, which aims to select a number of parameters for individual vaccines or intermediate products to prove batch-to-batch consistency.

Besides antigens, the inactivated poultry vaccines in this study contained mineral oil adjuvants in w/o formulation, which has been shown to form a depot with a low decomposition rate in vivo, leading to slow release kinetics of the antigen $[51,52]$. In our study, some vaccines enhanced bead uptake by HDII cells, whereas other vaccines did not, depending on the manufacturer of the vaccines. These results may be explained by differences in the antigen dose or release kinetics of the different products, which depends on the product ingredients and the manufacturing process. Light microscopy showed the accumulation of lipid droplets in HDI I cells upon stimulation with emulsified mineral oil or vaccines containing emulsified mineral oil. We used flow cytometric SSC, which measured cell contents and granularity [39], to assess quantitative differences in the accumulation of lipid droplets as a read-out for decomposition of the vaccines. Although, SSC increased upon incubation with higher concentrations of the individual vaccines, this did not always result in increased bead uptake. This suggests that the variation in phagocytosis between the vaccines cannot solely be explained by differences in decomposition rates. Therefore, phagocytosis may be affected by other determinants of the vaccine formulation, including differences in antigen content, the immunostimulatory capacity of the allantoic fluid, or cytotoxicity.

Since CHIR-ABI is known as a high-affinity chicken IgY Fc receptor that signals through Fc $\&$ receptor I gamma chain (Fc\&RI $\gamma$ ) upon interaction with heat-aggregated IgY [29], we investigated its involvement in the uptake of IgY-opsonized beads by HDII cells upon stimulation. We observed increased CHIR-ABI expression on HDI I cells upon stimulation with LPS and inactivated IBV antigen. In addition, blocking CHIR-ABI diminished the increase in phagocytosis as a result of stimulation. Hence, it must be concluded that inactivated IBV antigen led to an increase in phagocytosis due to an increase in the expression of CHIR-ABI. The increase in phagocytosis was therefore Fc receptor dependent. In a previous study, LPS was not found to affect CHIR-ABI expression by primary macrophages, which contrasts to our findings in the HDI I cell line [29]. The difference may also be explained by the use of another type of LPS.

The nitric oxide production assay and the phagocytosis assay presented in this study showed different levels of macrophage activity upon stimulation with individual inactivated poultry vaccines. Based on our results, nitric oxide production by HDII cells seemed to be 
(a)

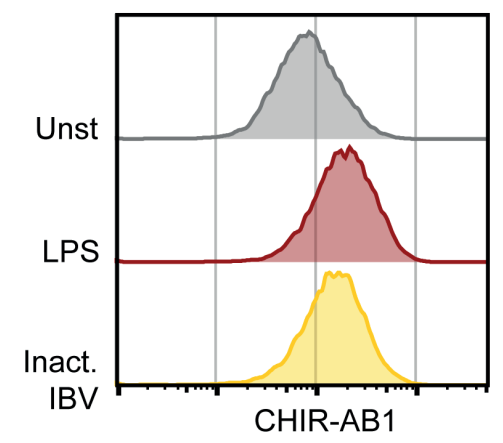

(c)

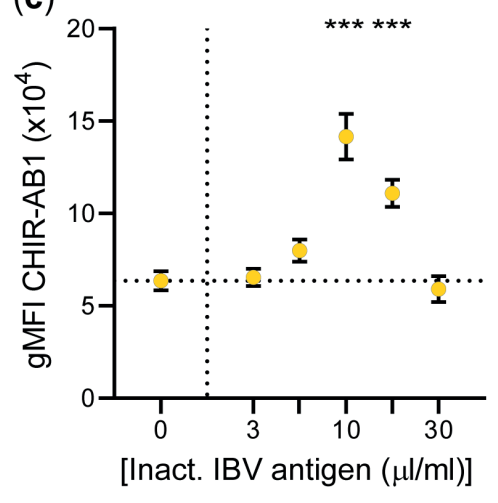

(b)

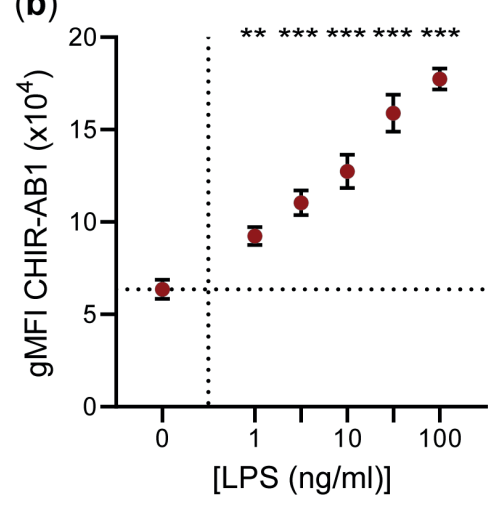

(d)

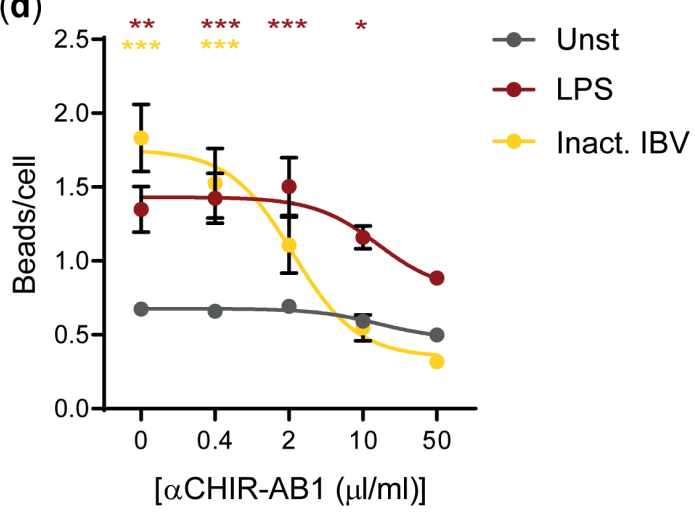

$n=6$ for all experimental groups of this figure

Figure 5. The induction of phagocytosis after exposure to inactivated IBV antigen is dependent on the IgY Fc receptor CHIR-ABI. (a) Representative histograms show CHIR-ABI expression by HDI I cells after $24 \mathrm{~h}$ without stimulation, stimulation with $100 \mathrm{ng} / \mathrm{mL}$ LPS, or stimulation with $10 \mu \mathrm{L} / \mathrm{mL}$ IBV antigen. (b,c) CHIR-ABI surface expression by HDII cells was quantified and expressed as the geometric mean fluorescent intensity (gMFI) after $24 \mathrm{~h}$ stimulation with different concentrations of LPS (b) and IBV antigen (c). (d) Unstimulated HDII cells and HDII cells stimulated with LPS or inactivated IBV antigen for $24 \mathrm{~h}$ received the blocking antibody $8 \mathrm{D} I 2$ specific for chicken CHIR-ABI $10 \mathrm{~min}$ before the addition of IgY-opsonized beads. The average number of phagocytosed beads per HDII cell is shown for different concentrations of blocking antibody. Three independent experiments were performed, and the experimental conditions of each independent experiment were tested in duplicate. Error bars represent the SEM. The experimental groups were tested for statistically significant differences in CHIR-ABI expression or bead uptake between stimulated and unstimulated groups using one-way ANOVA tests and Holm-Sidak's multiple comparisons tests. Statistical significance is indicated by $* p<0.05, * * p<0.01$, and $* * * p<0.00 \mathrm{I}$.

induced by the mineral oil adjuvant, when present in a water-in-oil formulation. Fc receptormediated phagocytosis by HDII cells was found to be induced by allantoic fluid, which is present in inactivated IBV and NDV antigens of poultry vaccines. Nitric oxide production was not induced by allantoic fluid, as demonstrated by the absence of nitric oxide after exposure to IBV and NDV antigens containing allantoic fluid. In this exploratory study, these 
two assays were able to show different immunopotentiating properties of the vaccines and hence may be used in the future as complementary assays to test for immunostimulatory properties of vaccines. Allantoic fluid might function as an inherent adjuvant and facilitate the immune response, although this remains to be confirmed with in vivo experiments. Finally, the assays used in this study may be applied in the future as quality control tests for inactivated poultry vaccines. Future studies will be needed to address the capacity of these cell-based assays to evaluate vaccine batch-to-batch consistency and detect non-conforming batches, as compared to the animal-based quality control tests that are currently in place as gold standards. Obviously, the capacity of inactivated poultry vaccines to induce antigenspecific immunity requires additional assessments, like antigen quantification or stability, to ensure vaccine quality. The nitric oxide production and phagocytosis assays with HDII cells might contribute to future efforts to replace current in vivo vaccine batch-release quality tests for in vitro alternatives.

\section{Conclusions}

In this exploratory study we demonstrated that inactivated poultry vaccines are able to activate chicken innate immune cells in vitro. Stimulation of the chicken macrophage-like cell line HDII with the inactivated poultry vaccines resulted in the production of nitric oxide due the presence of mineral oil adjuvant. Furthermore, inactivated poultry vaccines enhanced Fc receptor-mediated phagocytosis due to the presence of allantoic fluid in the vaccine antigen preparations. Taken together, these two in vitro assays were able to show different immunopotentiating properties of the vaccines and hence may be used in the future as complementary assays to test for immunostimulatory properties of vaccines.

\section{Funding}

This research was funded by the Innovative Medicines Initiative 2 Joint Undertaking under grant agreement No I 15924 (VAC2VAC). This Joint Undertaking receives support from the European Union's Horizon 2020 research and innovation program and EFPIA. The contents of this article represent the authors' scientific conclusions and neither IMI nor the European Union, EFPIA, or any Associated Partners are responsible for any use that may be made of the information contained therein. 


\section{Supplementary Materials}

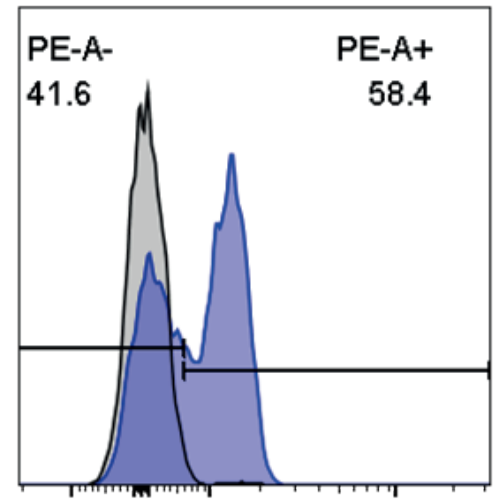

$\square \lg Y$ beads

IgY beads + MaCh lgY-PE

PE

Supplementary Figure SI. A major part of the beads used for the phagocytosis assay were coupled to IgY. An overlay of histograms shows IgY-coated beads without staining in grey and IgY-coated beads stained with a PE-conjugated mouse anti-chicken $\lg Y$ in blue.

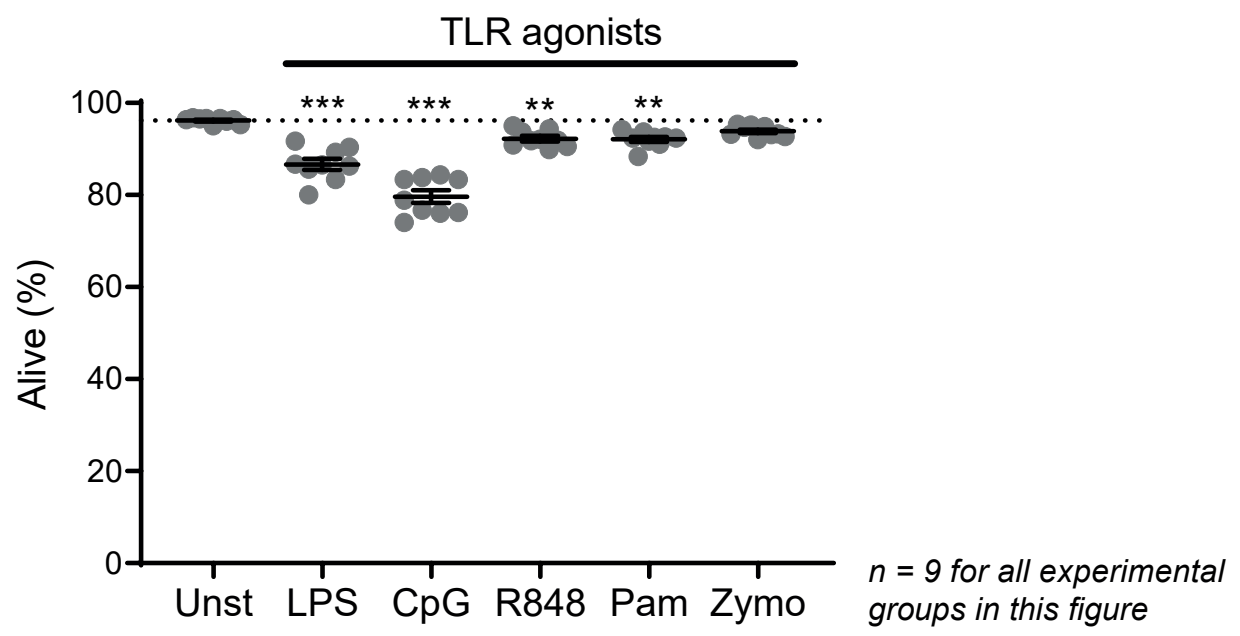

Supplementary Figure S2. Stimulation with TLR agonists showed little effects on HDII cell viability. The effect of TLR agonists on HDII cell viability, as determined by zombie aqua fixable/live dead staining, is expressed as the percentage of living cells (\%alive). HDI I cells were stimulated with

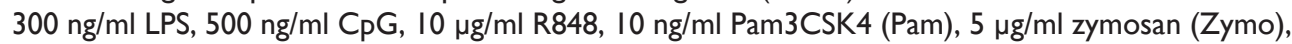
or left unstimulated (Unst). The data comprise three independent replicates performed in triplo. Error bars represent the SEM. The experimental groups were tested for statistically significant differences in viability between stimulated and unstimulated groups using a Kruskal-Wallis test and Dunn's multiple comparisons test. Statistical significance is indicated by $* p<0.05$, $* * p<0.01$ and $* * * p<0.001$. 


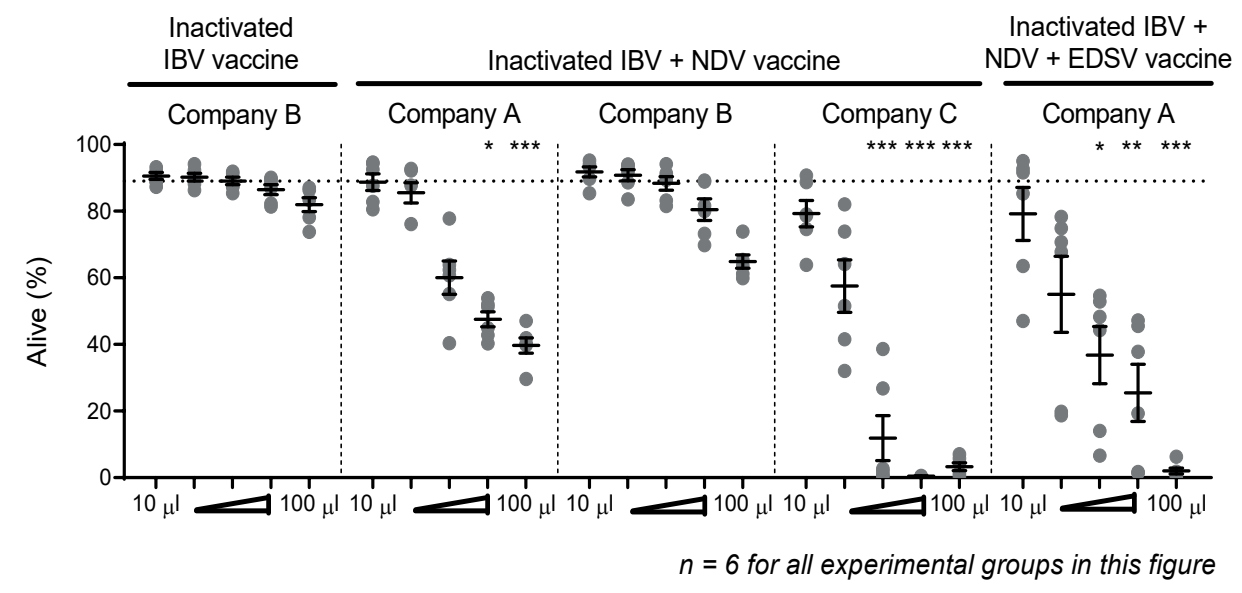

Supplementary Figure S3. The viability of HDI I cells was affected by various inactivating poultry vaccines to different extents. The effect of inactivated w/o vaccines on HDI I cell viability, as determined by zombie aqua fixable/live dead staining, is expressed as the percentage of living cells (\%alive). HDI I cells were stimulated with a monovalent vaccine (IBV) from company $B$, bivalent vaccines (IBV + NDV) from companies $A, B$, and $C$, and a trivalent vaccine (IBV + NDV + EDSV) from company $A$. The data comprise three independent replicates performed in duplo. Error bars represent the SEM. The experimental groups were tested for statistically significant differences in viability between stimulated and unstimulated groups using a Kruskal-Wallis test and Dunn's multiple comparisons test. Statistical significance is indicated by $* p<0.05$, $* * p<0.01$ and $* * * p<0.001$.

Supplementary Video SI. A 3D model of IgY-opsonized beads engulfed by HDI I cells. This movie is available online at https://www.mdpi.com/2076-393X/8/2/332/s I. 


\section{References}

I. Coffman RL, Sher A, Seder RA. Vaccine adjuvants: Putting innate immunity to work. Immunity (2010) 33(4):492-503. doi:10.1016/j.immuni.2010.10.002

2. Goddard RD, Nicholas RAJ, Luff PR. Serology-based potency test for inactivated Newcastle disease vaccines. Vaccine (1988) 6(6):530-532. doi:10.1016/0264-4I0X(88)90106-5

3. EDQM. European Pharmacopoeia. 10th ed. Strasbourgh, France: European Department for the Quality of Medicines; 2020.

4. Schutte K, Szczepanska A, Halder M, Cussler K, Sauer UG, Stirling C, et al. Modern science for better quality control of medicinal products "Towards global harmonization of $3 R s$ in biologicals": The report of an EPAA workshop. Biologicals (2017) 48:55-65. doi:10.1016/j. biologicals.2017.05.006

5. The European Parliament and the Council of the European Union. DIRECTIVE 2010/63/EU on the protection of animals used for scientific purposes. Off J Eur Union (2010) 276:33-79.

6. Hendriksen C, Arciniega JL, Bruckner L, Chevalier M, Coppens E, Descamps J, et al. The consistency approach for the quality control of vaccines. Biologicals (2008) 36(I):73-77. doi: 10.1016/j.biologicals.2007.05.002

7. Hendriksen CF. Replacement, reduction and refinement alternatives to animal use in vaccine potency measurement. Expert Rev Vaccines (2009) 8(3):3 I3-322. doi: I0.1586/ I4760584.8.3.3 I3

8. Bruysters MWP, Schiffelers MJ, Hoonakker M, Jungbaeck C, Ragan I, Rommel E, et al. Drivers and barriers in the consistency approach for vaccine batch release testing: Report of an international workshop. In: Biologicals. Vol 48. Academic Press; 20I7:I-5. doi:I0.1016/j.biologicals.2017.06.006

9. Claassen I, Maas R, Oei H, Daas A, Milne C. Validation study to evaluate the reproducibility of a candidate in vitro potency assay of newcastle disease vaccines and to establish the suitability of a candidate biological reference preparation. Pharmeuropa bio (2004) 2004(I): I-I5.

10. Maas PA, de Winter MPM, Venema S, Oei HL, Claassen IJTM. Antigen quantification as in vitro alternative for potency testing of inactivated viral poultry vaccines. Vet $Q 2000$. doi: $10.1080 / 01$ 652176.2000 .9695063

II. Maas R, Venema S, Kant A, Oei H, Claassen I. Quantification of infectious bursal disease viral proteins 2 and 3 in inactivated vaccines as an indicator of serological response and measure of potency. Avian Pathol (2004) 33(2):126-I32. doi: I0.1080/03079450310001652I2I

12. Stoel M, Pool J, de Vries-Idema J, Zaaraoui-Boutahar F, Bijl M, Andeweg AC, et al. Innate responses induced by whole inactivated virus or subunit influenza vaccines in cultured dendritic cells correlate with immune responses in vivo. PLoS One (2015) I0(5):e0I25228. doi:10.137I/ journal.pone.0125228

13. Hoonakker ME, Verhagen LM, Hendriksen CFM, van Els CACM, Vandebriel RJ, Sloots A, et al. In vitro innate immune cell based models to assess whole cell Bordetella pertussis vaccine quality: $A$ proof of principle. Biologicals (20I5) 43(2): 100-109. doi: I0.1016/j.biologicals.20I4.12.002

14. Marichal T, Ohata K, Bedoret D, Mesnil C, Sabatel C, Kobiyama K, et al. DNA released from dying host cells mediates aluminum adjuvant activity. Nat Med (201I) 17(8):996-1002. doi:10.1038/ nm.2403

15. Hayashi T, Momota M, Kuroda E, Kusakabe T, Kobari S, Makisaka K, et al. DAMP-inducing adjuvant and PAMP adjuvants parallelly enhance protective type-2 and type-I immune responses to influenza split vaccination. Front Immunol (20I8) 9(NOV). doi: 10.3389/fimmu.2018.026I9

16. Iqbal M, Philbin VJ, Smith AL. Expression patterns of chicken Toll-like receptor mRNA in tissues, immune cell subsets and cell lines. Vet Immunol Immunopathol (2005) I04(I-2): I I7- 27. doi:10.1016/J.VETIMM.2004.11.003

17. Crippen TL. The selective inhibition of nitric oxide production in the avian macrophage cell line HDII. Vet Immunol Immunopathol (2006) I09(I-2):I27-I37. doi:I0.1016/j.vetimm.2005.08.024

18. He H, Genovese KJ, Nisbet DJ, Kogut MH. Synergy of CPG oligodeoxynucleotide and doublestranded RNA (poly l:C) on nitric oxide induction in chicken peripheral blood monocytes. Mol Immunol (2007) 44(I2):3234-3242. doi:I0.10I6/j.molimm.2007.0I.034 
19. Peroval MY, Boyd AC, Young JR, Smith AL, Muller M. A Critical Role for MAPK Signalling Pathways in the Transcriptional Regulation of Toll Like Receptors. PLoS One (20I3) 8(2):e5I 243. doi: I0.137I/journal.pone.005 I243

20. Lin YF, Deng MC, Tseng LP, Jiang PR, Jan TR, Hsieh Fl, et al. Adjuvant effect of liposome in chicken result from induction of nitric oxide. Biomed Mater (20II) 6(I). doi:10.1088/1748$6041 / 6 / 1 / 015011$

21. van Dijk A, van Eldik M, Veldhuizen EJA, Tjeerdsma-van Bokhoven HLM, de Zoete MR, Bikker FJ, et al. Immunomodulatory and Anti-Inflammatory Activities of Chicken Cathelicidin-2 Derived Peptides. PLoS One (2016) II (2):e0I47919. doi:I0.I37I/journal.pone.01479I9

22. He H, Genovese KJ, Kogut MH. Modulation of chicken macrophage effector function by $\mathrm{T}(\mathrm{H}) \mathrm{I} /$ $\mathrm{T}(\mathrm{H}) 2$ cytokines. Cytokine (20I I) 53(3):363-369. doi:10.1016/j.cyto.2010.12.009

23. Haq K, Elawadli I, Parvizi P, Mallick Al, Behboudi S, Sharif S. Interferon- $\gamma$ influences immunity elicited by vaccines against very virulent Marek's disease virus. Antiviral Res (20II) 90(3):218226. doi:10.1016/j.antiviral.201I.04.00I

24. Sekellick MJ, Lowenthal JW, O'Neil TE, Marcus PI. Chicken interferon types I and II enhance synergistically the antiviral state and nitric oxide secretion. J Interf Cytokine Res (1998) 18(6):4074I4. doi:10.1089/jir.1998.18.407

25. He H, Genovese KJ, Swaggerty CL, Nisbet DJ, Kogut MH. A comparative study on invasion, survival, modulation of oxidative burst, and nitric oxide responses of macrophages (HDII), and systemic infection in chickens by prevalent poultry Salmonella serovars. Foodborne Pathog Dis (2012) 9(I2): I I04-I I I0. doi:I0.1089/fpd.20I2.1233

26. Zhang P, Ding Z, Liu X, Chen Y, Li J, Tao Z, et al. Enhanced replication of virulent Newcastle disease virus in chicken macrophages is due to polarized activation of cells by inhibition of TLR7. Front Immunol (2018) 9(APR). doi: I0.3389/fimmu.20I8.00366

27. Lee C-C, Wu CC, Lin TL. Role of chicken melanoma differentiation-associated gene 5 in induction and activation of innate and adaptive immune responses to infectious bursal disease virus in cultured macrophages. Arch Virol (20I5) I60(I2):302I-3035. doi:I0.1007/s00705-0I5-26I2-y

28. Qi X, Liu C, Li R, Zhang H, Xu X, Wang J. Modulation of the innate immune-related genes expression in H9N2 avian influenza virus-infected chicken macrophage-like cells (HDII) in response to Escherichia coli LPS stimulation. Res Vet Sci (2017) III:36-42. doi:10.1016/j. rvsc.2016.11.008

29. Viertlboeck BC, Schweinsberg S, Hanczaruk MA, Schmitt R, Du Pasquier L, Herberg FW, et al. The chicken leukocyte receptor complex encodes a primordial, activating, high-affinity IgY Fc receptor. Proc Natl Acad Sci U S A (2007) 104(28): I I 7 I 8- I I 723. doi: I0.1 073/pnas.07020 I I 104

30. de Geus ED, Jansen CA, Vervelde L. Uptake of particulate antigens in a nonmammalian lung: phenotypic and functional characterization of avian respiratory phagocytes using bacterial or viral antigens. J Immunol (2012) I88(9):45 I6-4526. doi:I0.4049/jimmunol. I 200092

31. Naghizadeh M, Wattrang E, Kjærup RB, Bakke M, Shih S, Dalgaard TS. In vitro phagocytosis of opsonized latex beads by HDI I cells as a method to assess the general opsonization potential of chicken serum. Avian Pathol (2018) 47(5):479-488. doi:10.1080/03079457.20I8.1490006

32. Grabowska J, Lopez-Venegas MA, Affandi AJ, Den Haan JMM. CDI69+ macrophages capture and dendritic cells instruct: The interplay of the gatekeeper and the general of the immune system. Front Immunol (2018) 9(OCT). doi:I0.3389/fimmu.2018.02472

33. de Geus ED, Vervelde L. Regulation of macrophage and dendritic cell function by pathogens and through immunomodulation in the avian mucosa. Dev Comp Immunol (20I3) 4I(3):34I-35I. doi:10.1016/j.dci.2013.03.008

34. Canthaboo C, Xing D, Wei XQ, Corbel MJ. Investigation of role of nitric oxide in protection from Bordetella pertussis respiratory challenge. Infect Immun (2002) 70(2):679-684. doi:I0.I I28/ IAI.70.2.679-684.2002

35. Quattrocchi V, Langellotti C, Pappalardo JS, Olivera V, Di Giacomo S, van Rooijen N, et al. Role of macrophages in early protective immune responses induced by two vaccines against foot and mouth disease. Antiviral Res (201 I) 92(2):262-270. doi: I0.1016/j.antiviral.201 I.08.007 
36. He W, Chen CJ, Mullarkey CE, Hamilton JR, Wong CK, Leon PE, et al. Alveolar macrophages are critical for broadly-reactive antibody-mediated protection against influenza $A$ virus in mice. Nat Commun (2017) 8(I). doi:10.1038/s4I467-0I7-00928-3

37. Beug H, von Kirchbach A, Döderlein G, Conscience J-F, Graf T. Chicken hematopoietic cells transformed by seven strains of defective avian leukemia viruses display three distinct phenotypes of differentiation. Cell (1979) 18(2):375-390. doi:10.1016/0092-8674(79)90057-6

38. Ariaans MP, Matthijs MGR, van Haarlem D, van de Haar P, van Eck JHH, Hensen EJ, et al. The role of phagocytic cells in enhanced susceptibility of broilers to colibacillosis after Infectious Bronchitis Virus infection. Vet Immunol Immunopathol (2008) 123(3-4):240-250. doi:10.1016/J. VETIMM.2008.02.003

39. Stringer B, Imrich A, Kobzik L. Flow cytometric assay of lung macrophage uptake of environmental particulates. Cytometry (1995) 20(I):23-32. doi:10.1002/cyto.990200106

40. Schindelin J, Arganda-Carreras I, Frise E, Kaynig V, Longair M, Pietzsch T, et al. Fiji: an opensource platform for biological-image analysis. Nat Methods (2012) 9(7):676-682. doi:10.1038/ nmeth.2019

4I. Stils HF. Adjuvants and Antibody Production: Dispelling the Myths Associated with Freund's Complete and Other Adjuvants. ILAR J (2005) 46(3):280-293. doi:I 0.1093/ilar.46.3.280

42. Philbin VJ, lqbal M, Boyd Y, Goodchild MJ, Beal RK, Bumstead N, et al. Identification and characterization of a functional, alternatively spliced Toll-like receptor 7 (TLR7) and genomic disruption of TLR8 in chickens. Immunology (2005) II4(4):507-52I. doi:10.1III/j.13652567.2005.02125.x

43. Brownlie R, Zhu J, Allan B, Mutwiri GK, Babiuk LA, Potter A, et al. Chicken TLR2I acts as a functional homologue to mammalian TLR9 in the recognition of $C_{P G}$ oligodeoxynucleotides. Mol Immunol (2009) 46(I5):3 I63-3 I70. doi: I0.10 I6/j.molimm.2009.06.002

44. Herrera-Rodriguez J, Signorazzi A, Holtrop M, de Vries-Idema J, Huckriede A. Inactivated or damaged? Comparing the effect of inactivation methods on influenza virions to optimize vaccine production. Vaccine (2019) 37(12): I630-1637. doi:10.1016/j.vaccine.2019.01.086

45. Geeraedts F, Goutagny N, Hornung V, Severa M, de Haan A, Pool J, et al. Superior Immunogenicity of Inactivated Whole Virus H5NI Influenza Vaccine is Primarily Controlled by Toll-like Receptor Signalling. PLoS Pathog (2008) 4(8):el000 I38. doi: I0.137I/journal.ppat. 1000138

46. Bolin G, Burggren WW. Metanephric kidney development in the chicken embryo: Glomerular numbers, characteristics and perfusion. Comp Biochem Physiol - A Mol Integr Physiol (2013) I66(2):343-350. doi:10.1016/j.cbpa.2013.07.01।

47. Marrack P, McKee AS, Munks MW. Towards an understanding of the adjuvant action of aluminium. Nat Rev Immunol (2009) 9(4):287-293. doi:10.1038/nri25I0

48. Depelsenaire ACl, Meliga SC, McNeilly CL, Pearson FE, Coffey JW, Haigh OL, et al. Colocalization of cell death with antigen deposition in skin enhances vaccine immunogenicity. J Invest Dermatol (20I4) I34(9):236I-2370. doi:I0.1038/jid.20I4.I74

49. Yang Y-W, Shen S-S. Enhanced antigen delivery via cell death induced by the vaccine adjuvants. Vaccine (2007) 25(45):7763-7772. doi:I 0.10I6/J.VACCINE.2007.08.064

50. Behrens MD, Wagner WM, Krco CJ, Erskine CL, Kalli KR, Krempski J, et al. The endogenous danger signal, crystalline uric acid, signals for enhanced antibody immunity. Blood (2008) I I I(3): I472-I479. doi:I0.1 I82/blood-2007-10-II7I84

5I. Droual R, Bickford AA, Charlton BR, Kuney DR. Investigation of problems associated with intramuscular breast injection of oil-adjuvanted killed vaccines in chickens. Avian Dis (1990) 34(2):473-478. doi:10.2307/I59/439

52. Yamanaka M, Okabe T, Nakai M, Goto N. Local pathological reactions and immune response of chickens to ISA-70 and other adjuvants containing Newcastle disease virus antigen. Avian Dis (I993) 37(2):459-466. doi:10.2307/I59/673 


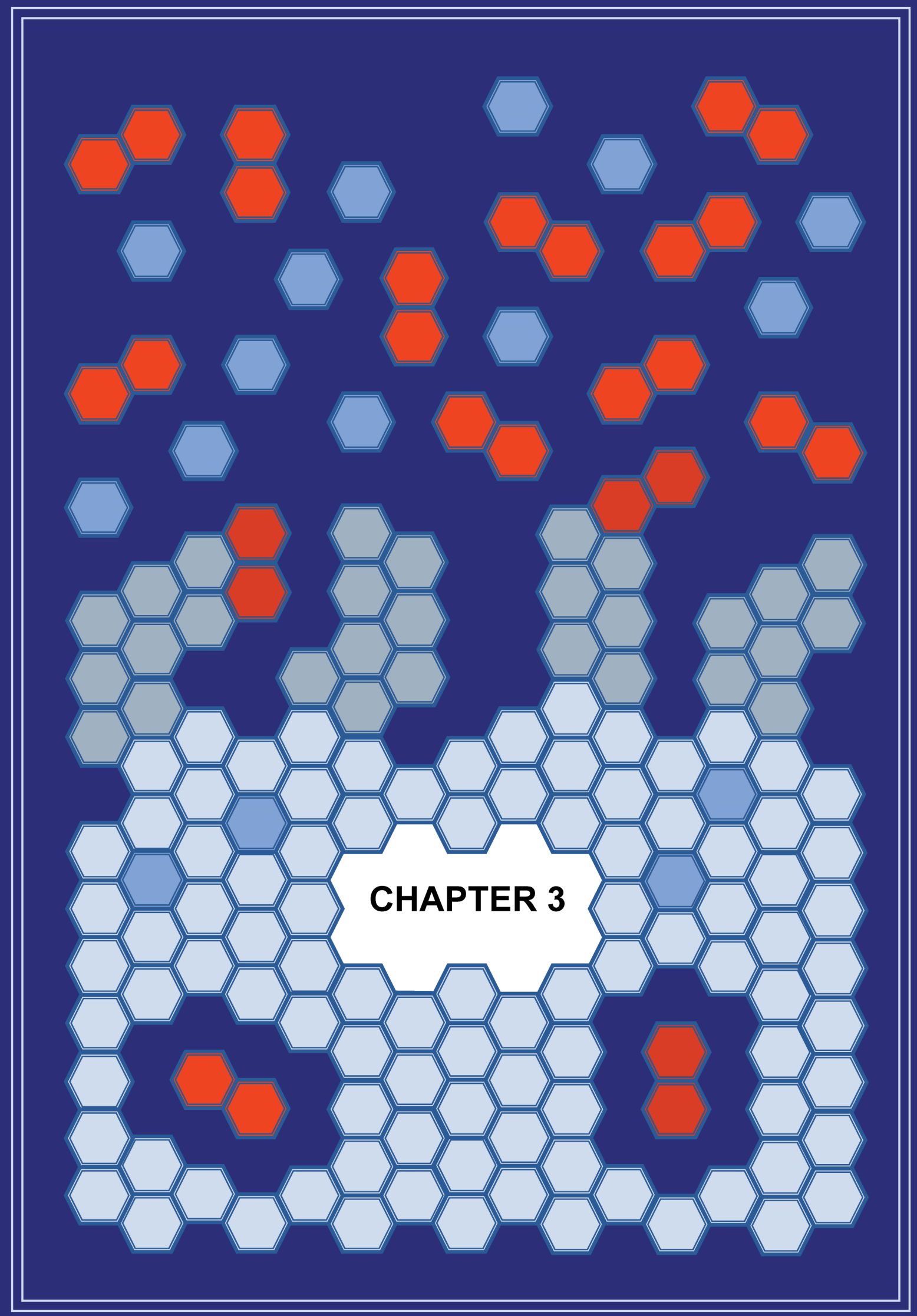


Macrophage activation assays to evaluate the immunostimulatory capacity of Avibacterium paragallinarum in a multivalent poultry vaccine

Robin H.G.A. van den Biggelaar', Willem van Eden', Victor P.M.G. Rutten ${ }^{1,2}$ and Christine A. Jansen'

'Division of Infectious Diseases and Immunology, Department of Biomolecular Health Sciences, Faculty of Veterinary Medicine, Utrecht University, Utrecht, the Netherlands

${ }^{2}$ Department of Veterinary Tropical Diseases, Faculty of Veterinary Science, University of Pretoria, Pretoria, South Africa

Vaccines 2020, 8(4), 671

https://doi.org// 0.3390/vaccines8040671 
Chapter 3 | The immunostimulatory capacity of $A v$. paragallinarum in multivalent vaccines

\section{Abstract}

High-quality vaccines are crucial to prevent infectious disease outbreaks in the poultry industry. In vivo vaccination tests are routinely used to test poultry vaccines for their potency, i.e., their capacity to induce protection against the targeted diseases. A better understanding of how poultry vaccines activate immune cells will facilitate the replacement of in vivo potency tests for in vitro assays. Using the chicken macrophage-like HDI I cell line as a model to evaluate innate immune responses, the current explorative study addresses the immunostimulatory capacity of an inactivated multivalent vaccine for infectious bronchitis, Newcastle disease, egg-drop syndrome, and infectious coryza. The vaccine stimulated HDI I cells to produce nitric oxide and to express pro-inflammatory cytokines $I L-I \beta, T N F$, and IL-I $2 p 40$, chemokines CXCLil and CXCLi2, and the anti-inflammatory cytokine IL-IO, but only when inactivated Avibacterium paragallinarum, the causative agent of infectious coryza, was present. Lipopolysaccharides from Avibacterium paragallinarum were crucial for the production of nitric oxide and expression of $I L-I \beta$ and CXCLil. The described immune parameters demonstrate the capacity of this multivalent vaccine to activate innate immune cells and may in the future, combined with antigen quantification methods, contribute to vaccine quality testing in vitro, hence the replacement of current in vivo vaccination tests. 


\section{Introduction}

In the poultry industry, chickens are routinely vaccinated against infectious diseases to maintain flock health. Vaccine batches are subjected to routine quality control (QC) to ensure their potency, i.e., their capacity to induce protection against the targeted infectious diseases. For many poultry vaccines, current $\mathrm{QC}$ test approaches include in vivo vaccination-challenge or vaccination-serology tests, which require large numbers of chickens. Alternatively, poultry vaccines could be tested in accordance with the consistency approach [I], which implies that quality can be proven by in vitro methods that demonstrate the similarity between the product profiles of a new batch and reference batches of proven clinical efficacy and safety. The characteristics that determine the potency of a vaccine have to be predetermined and may include properties like antigen quantity and the capacity to stimulate innate immune responses $[\mathrm{I}, 2,3]$.

Infectious coryza is an upper respiratory tract infection caused by the Gram-negative bacterium Avibacterium (Av.) paragallinarum, which is responsible for worldwide economic losses due to mortality and reduced egg production in chickens [4]. Breeding and laying hens can be protected against infectious coryza by inactivated vaccines comprising multiple $A v$. paragallinarum isolates of serovars $A, B$, and $C$ [5]. Like the vaccine used in this study, $A v$. paragallinarum can be incorporated in inactivated multivalent vaccines that also comprise infectious bronchitis virus (IBV), egg-drop syndrome '76 virus (EDSV) and Newcastle disease virus (NDV), to reduce the time and costs of vaccination [6]. Inactivated NDV vaccines can be assessed for potency using an in vitro enzyme-linked immunosorbent assay (ELISA) $[7,8,9]$ and inactivated IBV and EDSV vaccines can be assessed for potency by in vivo vaccination-serology tests $[\mathrm{I} 0, \mathrm{II}]$, which have a lower impact on animal welfare than traditional vaccination-challenge tests. In contrast, inactivated infectious coryza vaccines still rely on vaccination-challenge tests to prove their potency and are hence in need of reliable in vitro alternatives $[12,13]$. The capacity of a vaccine to induce protective immunity depends in part on its capacity to stimulate innate immune cells through recognition of immunostimulatory constituents including adjuvants and intrinsic pathogen-associated molecular patterns (PAMPs) like bacterial cell wall components $[14,15]$. The importance of PAMPs for effective vaccination has already been demonstrated for human vaccines against bacterial pathogens [16] like Bordetella pertussis [17,18] and Mycobacterium tuberculosis $[14,19]$. However, these immunostimulatory constituents have not been described for most poultry vaccines. Here, we investigated whether infectious coryza vaccines contain PAMPs that activate innate immune cells.

In general, bacterial PAMPs are recognized by innate immune cells through pattern recognition receptors (PRRs), which are broadly expressed by phagocytes like macrophages. Furthermore, the importance of macrophages in detecting bacterial PAMPs during vaccination has been demonstrated in macrophage-depletion studies in mice [20], which justifies the use of this cell type in in vitro vaccine quality assessment. A prime candidate to characterize innate immune responses evoked by poultry vaccines is the chicken macrophage-like cell line HDII. The HDII cell line was originally described as LSCC-HD (MC/MAI) [2I] but later renamed to HDII [22]. This cell line recognizes bacteria using a broad repertoire of PRRs, including most chicken toll-like receptors (TLRs) [23] and mannose receptors. Activation of HDII cells can be observed by increased phagocytosis [24,25,26], cytokine 
expression [27,28], and nitric oxide production by the inducible nitric oxide synthase (iNOS) $[27,28,29,30,31]$. As vaccines for Av. paragallinarum are commonly multivalent and also used to protect chickens against other pathogens, we used two multivalent vaccines in the current study. To identify which responses were directed against $A v$. paragallinarum, an octavalent vaccine comprising inactivated IBV, NDV, EDSV, and 5 strains of Av. paragallinarum was used next to a trivalent vaccine that only contained inactivated IBV, NDV, and EDSV. In this explorative study, we aimed to identify candidate immune parameters for an in vitro QC test for inactivated poultry vaccines against $A v$. paragallinarum and contribute to the replacement of current in vivo vaccination-challenge tests.

\section{Materials and Methods}

\section{HD I I cell culture and stimulation}

Batches of chicken macrophage-like HDII cells [2I] were suspended in complete Roswell Park Memorial Institute (RPMI)-1640 medium with 50\% FBS (both from Gibco, Life Technologies Limited, Paisley, UK) and 10\% DMSO (Honeywell, Bucharest, Romania) and stored at $-140{ }^{\circ} \mathrm{C}$. After thawing, they were maintained and propagated in a complete RPMI-I640 cell culture medium supplemented with GlutaMAX-I, phenol red, HEPES, I0\% FBS, $200 \mathrm{U} / \mathrm{mL}$ penicillin, and $200 \mathrm{U} / \mathrm{mL}$ streptomycin (all from Gibco) at $37{ }^{\circ} \mathrm{C}, 5 \% \mathrm{CO}_{2}$, in Corning $75 \mathrm{~cm} 2$ cell culture flasks (Sigma-Aldrich, Saint Louis, MO, USA). The cells were passaged twice weekly by washing the cells in Dulbecco's phosphate-buffered saline without calcium and magnesium (PBS; Lonza, Basel, Switzerland) and detaching adherent cells using a $0.25 \%$ trypsin/EDTA solution supplemented with phenol red (Gibco). For the experiments, HDI I cells were harvested after 3 to 20 passages using the trypsin/EDTA solution, counted, and resuspended at a concentration of 200,000 cells $/ \mathrm{mL}$ in complete RPMI- 1640 medium. The cells were seeded at I mL/well in Corning Costar 24-well cell culture plates (SigmaAldrich) and cultured overnight at $37^{\circ} \mathrm{C}$ and $5 \% \mathrm{CO}_{2}$.

HDII cells were exposed to various stimuli to assess their activation using nitric oxide production as a readout. First of all, TLR agonists were given at eleven different concentrations with increments of 100.25 between consecutive concentrations to create dose-response curves and to test the ability of HDII cells to recognize different PAMPs. Stimuli included I.0-300 ng/mL lipopolysaccharides (LPS) from Escherichia (E.) coli OI27:B8 (Sigma-Aldrich) to target TLR4, 0.2-60 ng/mL Pam3CSK4 to target the TLR2/I heterodimer, $0.2-60 \mu \mathrm{g} / \mathrm{mL}$ zymosan from Saccharomyces (S.) cerevisiae to target the TLR2/6 heterodimer, 0.1-30 $\mathrm{gg} / \mathrm{mL}$ high molecular weight (I.5-8kb) polyinosinic-polycytidylic acid (poly[l:C]) to target TLR3, $0.1-30 \mu \mathrm{g} / \mathrm{mL}$ resiquimod (R848) to target TLR7, and 3-1000 ng/mL CpG oligonucleotides (ODN) 2006 to target TLR2I (all InvivoGen, San Diego, CA, USA). In addition, HDII cells were exposed to established tri- an octavalent inactivated poultry vaccines kindly provided by Boehringer Ingelheim (Ingelheim am Rhein, Germany) as a partner of the VAC2VAC consortium (http://www.vac2vac.eu/). The octavalent vaccine comprised of whole-inactivated IBV, NDV, EDSV, and inactivated isolates of five serovars of $A v$. paragallinarum, including one isolate of serovar $A$, three of serovar $B$, and one of serovar C. The trivalent vaccine used in this study comprised whole-inactivated IBV, NDV, and EDSV only. Both vaccines were adjuvanted as mineral oil-based water-in-oil emulsions. The vaccines were prepared such that a single chicken vaccination dose corresponded to $0.5 \mathrm{~mL}$ vaccine. HDI I cells were exposed to the vaccines at a fixed concentration of $\mathrm{I} \mu \mathrm{L} / \mathrm{mL}$ or 
to different concentrations ranging from 0.2 to $30 \mu \mathrm{L} / \mathrm{mL}$ to create dose-response curves.

In accordance with a previous publication [7], total antigenic fractions were extracted from the vaccines by adding vaccine to isopropyl myristate (Sigma-Aldrich) in a I:5 ratio, vigorous mixing on a vortex mixer for I min, and centrifugation at $1000 x \mathrm{~g}$. The lower water phase contained the antigenic fraction, which was gently resuspended and collected. HDII cells were exposed to extracted antigens at a fixed concentration of $0.5 \mu \mathrm{L} / \mathrm{mL}$ or different concentrations ranging from $0.2-30 \mu \mathrm{L} / \mathrm{mL}$ to create dose-response curves. Furthermore, Av. paragallinarum bacteria were purified from $200 \mu \mathrm{L}$ extracted antigens by centrifuging three times at 15,000 $\mathrm{g}$ and washing the bacterial pellet in PBS. Finally, the bacterial pellet was resuspended in $200 \mu \mathrm{L}$ PBS. A pellet was absent when the trivalent vaccine comprising only viral antigens was exposed to the same procedure. HDI I cells were exposed to the purified bacteria at different concentrations ranging from $0.1-10 \mu \mathrm{L} / \mathrm{mL}$ to create doseresponse curves.

The contribution of LPS, present within the antigens extracted from the octavalent vaccine, to the activation of HDI I cells was determined using the LPS-binding antibiotic polymyxin B (Sigma-Aldrich) [32]. For these experiments, $300 \mathrm{ng} / \mathrm{mL}$ E. coli LPS, $300 \mathrm{ng} /$ $\mathrm{mL}$ CpG ODN2006, $0.5 \mu \mathrm{L} / \mathrm{mL}$ octavalent vaccine and $1.0 \mu \mathrm{L} / \mathrm{mL}$ extracted antigens were resuspended in complete RPMI- 1640 and pre-incubated with I, I0, or $100 \mu \mathrm{g} / \mathrm{mL}$ polymyxin $\mathrm{B}$ for I h at $37^{\circ} \mathrm{C}$. Subsequently, these mixtures were added to the HDI I cells and incubated for $48 \mathrm{~h}$ to evaluate the production of nitric oxide or for different periods of time between $0-48 \mathrm{~h}$ to evaluate the gene expression of candidate biomarkers over time.

\section{Griess test to evaluate nitric oxide production by stimulated HD I I cells} Nitric oxide production by HDII cells was measured after $48 \mathrm{~h}$ of stimulation by the Griess test as previously described [33]. Griess test reagent was made by dissolving N-(Inaphtyl)ethylenediamine at $3 \mathrm{~g} / \mathrm{L}$ and sulfanilamide at $10 \mathrm{~g} / \mathrm{L}$ (both from Sigma-Aldrich) in 2.5\% phosphoric acid (Supelco, Merck, St. Louis, MO, USA) and mixing the two solutions I:I. From the stimulated HDI I cell cultures, $50 \mu \mathrm{L}$ supernatant was harvested, transferred to a 96-well flat-bottom plate (Corning B.V. Life Sciences, Amsterdam, the Netherlands), and mixed with $50 \mu \mathrm{L} /$ well Griess test reagent. The mixture turns purple upon reaction with nitrite ions in the cell culture supernatant. The median optical density at $540 \mathrm{~nm}$ was determined for each well using a FLUOstar Omega microplate reader (BMG Labtech, Ortenberg, Germany). The corresponding nitrite concentrations were calculated according to a nitrite standard curve made from a dilution series between 3.13-200 $\mu$ M sodium nitrite (Sigma-Aldrich). For HDI I stimulated with TLR agonists or purified inactivated $A v$. paragallinarum bacteria, the results of the Griess test were plotted using the four-parameter logistic regression model from GraphPad Prism 8 software (GraphPad Software)

$$
E(C)=E_{\max }+\frac{E_{\max }-E_{\min }}{1+\left(c / E C_{50}\right)^{n}}
$$

where $\mathrm{E}(\mathrm{C})$ = effect at a given concentration $(\mathrm{C}), \mathrm{E}_{\max }=$ maximum observed effect, $\mathrm{E}_{\min }$ $=$ minimum observed effect, $\mathrm{EC}_{50}=$ half-maximum effective concentration, and $\mathrm{n}=$ Hill 
coefficient. The model was used to determine the maximum nitric oxide production $\left(\mathrm{E}_{\max }\right)$ and the concentration that gives half-maximal nitric oxide production $\left(\mathrm{EC}_{50}\right)$ as readouts for the capacity of the stimuli to induce nitric oxide production.

\section{Relative expression of iNOS and cytokines using real-time quantitative PCR}

To determine iNOS and cytokine gene expression, HDII cells were harvested, either 8 $\mathrm{h}$ after stimulation or at different time points between $0-48 \mathrm{~h}$ (as indicated in Figure 3), using $200 \mu \mathrm{L}$ PBS $+5 \mathrm{mM}$ UltraPure EDTA (Invitrogen ${ }^{\mathrm{TM}}$, Life Technologies Europe BV, Bleiswijk, the Netherlands) and centrifugated at 400x g. The supernatant was discarded, and the pelleted cells were lysed in RLT buffer (Qiagen GmbH, Hilden, Deutschland) and stored at $-20^{\circ} \mathrm{C}$ until further processing. After thawing, RNA isolation was performed with the RNeasy Mini Kit (Qiagen GmbH, Hilden, Deutschland) including a DNase treatment using the RNase-Free DNase Set (Qiagen GmbH, Hilden, Deutschland). Next, cDNA was prepared using the iScript cDNA Synthesis Kit (Bio-Rad Laboratories B.V., Veenendaal, the Netherlands) according to the manufacturer's instructions. RT-qPCR reactions were performed with 100 nM FAM-TAMRA-labelled TaqMan probes (listed in Table I) together with $600 \mathrm{nM}$ primers and TaqMan Universal PCR Master Mix or without probes using 400 nM primers and SYBR Green Master Mix (all from Applied Biosystems, Life Technologies Europe BV, Bleiswijk, the Netherlands). RT-qPCR reactions were performed using a CFX Connect qPCR detection system (Bio-Rad), using a program of 40 cycles with melting temperatures of 58 (SYBR Green probes) or $59^{\circ} \mathrm{C}$ (TaqMan probes), and analyzed with CFX Maestro software (Bio-Rad). All RT-qPCR reactions were evaluated for proper amplification efficiency (95-105\%) using serial dilutions of reference cDNA or plasmids before testing the samples (data not shown). RT-qPCR reactions were performed in triplicate for every sample. Changes in gene expression over time upon stimulation were assessed using $\mathrm{t}=0 \mathrm{~h}$ as a reference time point and expressed as $2^{-\Delta \Delta C t}$-values, according to the Livak method [34] with $\mathrm{Ct}$ being the number of cycles before a signal above the threshold (background) level was reached. The results were normalized to gene expression levels of the housekeeping genes $28 \mathrm{~S}$ and GAPDH. An exception is the IL- 10 gene expression data of Supplementary Figure S2, which were expressed as $40-\mathrm{Ct}$ values according to a previous publication [35] as this method was more suitable when one or more data points did not reach a signal above the threshold within 40 cycles.

\section{Flow cytometric assessment of HDI I cell viability after stimulation}

HDII cells were harvested $48 \mathrm{~h}$ after stimulation using $200 \mu \mathrm{L}$ PBS $+5 \mathrm{mM}$ UltraPure EDTA and centrifuged at $400 \times \mathrm{g}$. The cells were transferred to a 96-well V-bottom plate, washed in PBS with $0.5 \%$ bovine serum albumin, $0.005 \%$ sodium azide (FACS buffer; both from Sigma-Aldrich), and stained for viability in $200 \mu \mathrm{L}$ FACS buffer with $1.25 \mu \mathrm{g} / \mathrm{mL}$ 7-aminoactinomycin D (7-AAD; BD Biosciences, Pharmingen, San Diego, CA, USA). The staining of the cells was analyzed using the $488 \mathrm{~nm}$ laser of a CytoFLEX LX flow cytometer (Beckman Coulter Inc., Brea, CA, USA). The fraction of viable 7-AAD-negative HDII cells was determined using Flowjo Software v. I0.5 (Flowjo LCC, Ashland, OR, USA). The cytotoxicity of the extracted antigens and purified bacteria from the octavalent vaccine was expressed as the concentration at which $50 \%$ of $\mathrm{HDII}$ cells had died, i.e., the $50 \%$ lethal concentration $\left(\mathrm{LC}_{50}\right)$. 
Table I. Primer and probe sequences. All probes contain the $5^{\prime}$ reporter dye FAM and the $3^{\prime}$ fluorescent quencher TAMRA.

\begin{tabular}{|c|c|c|c|}
\hline Gene & $\begin{array}{l}\text { NCBI } \\
\text { Reference }\end{array}$ & Type & Sequences $\left(5^{\prime}-3^{\prime}\right)$ \\
\hline \multirow{2}{*}{ iNOS } & \multirow{2}{*}{ NM_20496I.I } & Forward & TGGGTGGAAGCCGAAATA \\
\hline & & Reverse & GTACCAGCCGTTGAAAGGAC \\
\hline \multirow{2}{*}{ TNF } & \multirow{2}{*}{ MF000729.I } & Forward & CGCTCAGAACGACGTCAA \\
\hline & & Reverse & GTCGTCCACACCAACGAG \\
\hline \multirow{2}{*}{ CXCLil } & \multirow{2}{*}{ NM_205018.I } & Forward & CCAGTGCATAGAGACTCATTCCAAA \\
\hline & & Reverse & TGCCATCTTTCAGAGTAGCTATGACT \\
\hline \multirow{2}{*}{ GAPDH } & \multirow{2}{*}{ NM_204305.I } & Forward & GTGGTGCTAAGCGTGTTATC \\
\hline & & Reverse & GCATGGACAGTGGTCATAAG \\
\hline \multirow{3}{*}{$I L-I \beta$} & \multirow{3}{*}{ NM_204524.I } & Forward & GCTCTACATGTCGTGTGTGATGAG \\
\hline & & Reverse & TGTCGATGTCCCGCATGA \\
\hline & & Probe & CCACACTGCAGCTGGAGGAAGCC \\
\hline \multirow{3}{*}{ IL-4 } & \multirow{3}{*}{ NM_001007079.I } & Forward & AACATGCGTCAGCTCCTGAAT \\
\hline & & Reverse & TCTGCTAGGAACTTCTCCATTGAA \\
\hline & & Probe & AGCAGCACCTCCCTCAAGGCACC \\
\hline \multirow{3}{*}{ IL-6 } & \multirow{3}{*}{ NM_204628.I } & Forward & GCTCGCCGGCTTCGA \\
\hline & & Reverse & GGTAGGTCTGAAAGGCGAACAG \\
\hline & & Probe & AGGAGAAATGCCTGACGAAGCTCTCCA \\
\hline \multirow{3}{*}{ CXCLi2 } & \multirow{3}{*}{ NM_205498.I } & Forward & GCCCTCCTCCTGGTTTCA \\
\hline & & Reverse & TGGCACCGCAGCTCATT \\
\hline & & Probe & TCTTTACCAGCGTCCTACCTTGCGACA \\
\hline \multirow{3}{*}{ IL-10 } & \multirow{3}{*}{ NM_0010044|4.2 } & Forward & CATGCTGCTGGGCCTGAA \\
\hline & & Reverse & CGTCTCCTTGATCTGCTTGATG \\
\hline & & Probe & CGACGATGCGGCGCTGTCA \\
\hline \multirow{3}{*}{ IL-I $2 p 35$} & \multirow{3}{*}{ NM_213588.I } & Forward & TGGCCGCTGCAAACG \\
\hline & & Reverse & ACCTCTTCAAGGGTGCACTCA \\
\hline & & Probe & CCAGCGTCCTCTGCTTCTGCACCTT \\
\hline \multirow{3}{*}{ IL-I $2 p 40$} & \multirow{3}{*}{ NM_21357I.I } & Forward & TGGGCAAATGATACGGTCAA \\
\hline & & Reverse & CTGAAAAGCTATAAAGAGCCAAGCAAGACGTTCT \\
\hline & & Probe & CAGAGTAGTTCTTTGCCTCACATTTT \\
\hline \multirow{3}{*}{ IFN- $\alpha$} & \multirow{3}{*}{ XM_0I5277440.2 } & Forward & GACAGCCAACGCCAAAGC \\
\hline & & Reverse & GTCGCTGCTGTCCAAGCATT \\
\hline & & Probe & CTCAACCGGATCCACCGCTACACC \\
\hline \multirow{3}{*}{ IFN- $\gamma$} & \multirow{3}{*}{ NM_205I49.I } & Forward & GTGAAGAAGGTGAAAGATATCATGGA \\
\hline & & Reverse & GCTTTGCGCTGGATTCTCA \\
\hline & & Probe & TGGCCAAGCTCCCGATGAACGA \\
\hline \multirow{3}{*}{$28 \mathrm{~S}$} & \multirow{3}{*}{ XR_003078040.I } & Forward & GGCGAAGCCAGAGGAAACT \\
\hline & & Reverse & GACGACCGATTTGCACGTC \\
\hline & & Probe & AGGACCGCTACGGACCTCCACCA \\
\hline
\end{tabular}

\section{Statistical analysis}

Statistical analyses were performed using GraphPad Prism 8 software (GraphPad Software Inc., San Diego, CA, USA). When the assumptions of normally distributed data and residuals were met, a one-way ANOVA with Holm-Sidak's multiple comparisons test was used to test 
for statistically significant differences between stimulated and unstimulated control samples. Relative gene expression data were log-transformed to create normally distributed data. When the assumptions of normality were not met, a non-parametric Kruskal-Wallis test with Dunn's multiple comparisons test was used instead. A $p$-value of $<0.05$ was considered statistically significant.

\section{Results}

The inactivated octavalent poultry vaccine induces HDI I cells to produce nitric oxide

HDII cells were stimulated for $48 \mathrm{~h}$ by different TLR agonists derived from or mimicking components from bacteria (Pam3CSK4, LPS, CpG), viruses (R848, CpG, poly[l:C]), and fungi (zymosan). Nitric oxide production was analyzed using the Griess test (Figure I). The most potent inducer of nitric oxide production was Pam3CSK4 $\left(E_{50}=23.0 \mathrm{ng} / \mathrm{mL} ; \mathrm{E}_{\max }=\right.$ I0I $\mu \mathrm{M}$ nitrite), which stimulates the chicken TLR2/I heterodimer. Other potent stimuli were the TLR4 agonist $E$. coli LPS $\left(E_{50}=122 \mathrm{ng} / \mathrm{mL} ; \mathrm{E}_{\max }=93.5 \mu \mathrm{M}\right.$ nitrite $)$ and the TLR2 I

(a)

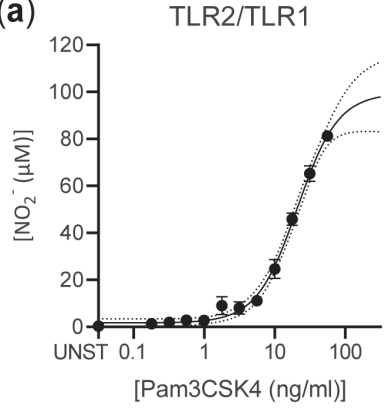

(d)

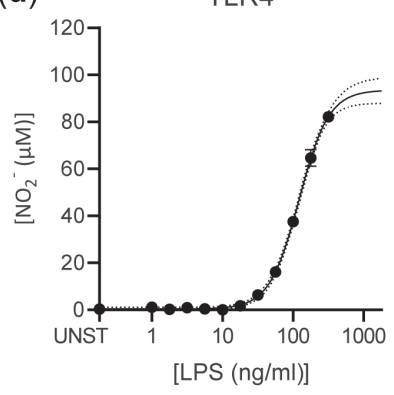

(b)

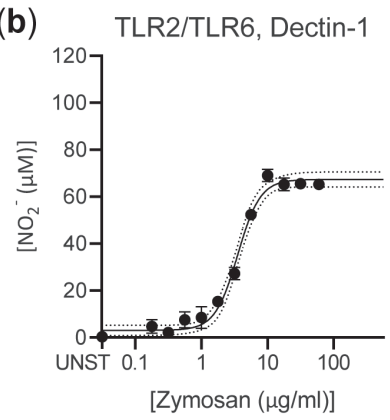

(e)

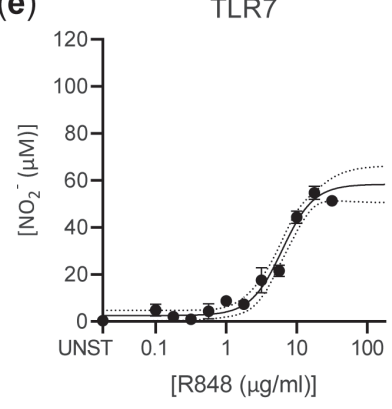

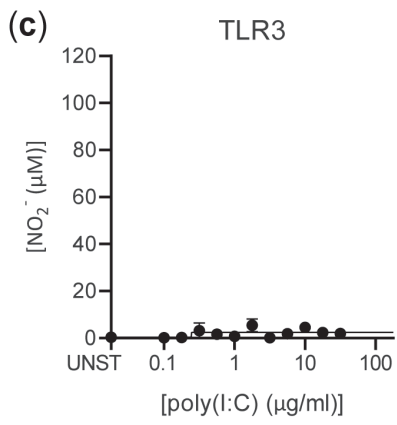

(f)

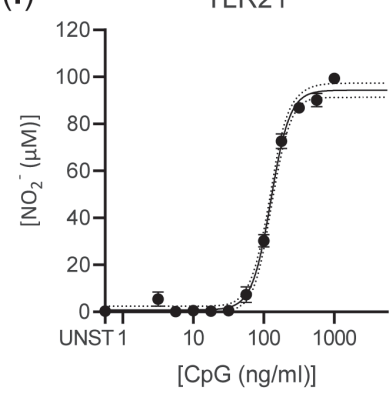

Figure I. The macrophage-like HDI I cell line produces nitric oxide upon stimulation with a broad range of TLR agonists for $48 \mathrm{~h}$. HDI I cells were stimulated with titrated concentrations of the TLR agonists Pam3CSK4 (a), zymosan (b), poly(l:C) (c), LPS (d), R848 (e), and CPG (f). The expected receptors for each agonist are shown above each panel. Nitric oxide production is expressed as the concentration of nitrite ions $\left(\mathrm{NO}_{2}^{-}\right)$in the cell culture supernatant, as measured by the Griess test. Four parameter logistic curves were plotted together with their confidence intervals (dotted lines). Unstimulated HDI I cells (UNST) were used as a negative control and HDI I cells stimulated with 300 $\mathrm{ng} / \mathrm{ml}$ LPS (d) were used as a positive control in this and all subsequent experiments. The experiment was performed in triplicate. The error bars represent the SEM. 
agonist CpG ODN2006 ( $\mathrm{EC}_{50}=127 \mathrm{ng} / \mathrm{mL} ; \mathrm{E}_{\max }=94.7 \mu \mathrm{M}$ nitrite). High concentrations of $\mathrm{R} 848\left(\mathrm{EC}_{50}=6.42 \mu \mathrm{g} / \mathrm{mL} ; \mathrm{E}_{\max }=57.1 \mu \mathrm{M}\right.$ nitrite), stimulating TLR7, and S. cerevisiae zymosan $\left(E_{50}=3.68 \mu \mathrm{g} / \mathrm{mL} ; E_{\max }=66.3 \mu \mathrm{M}\right.$ nitrite), stimulating both the TLR2/6 heterodimer and Dectin-I, were required to induce nitric oxide production. Nitric oxide was not detected upon exposure to high molecular weight poly $(\mathrm{l}: \mathrm{C})$ oligonucleotides. Overall, these results demonstrate that nitric oxide production can be used as a readout to determine the capacity of various compounds to stimulate innate immune cells like macrophages.

Next, we investigated whether an inactivated octavalent poultry vaccine for IBV, NDV, EDSV, and five serovars of $A v$. paragallinarum contains any immunostimulatory constituents that may stimulate nitric oxide production by HDI I cells. Stimulation with this vaccine for $48 \mathrm{~h}$ at concentrations ranging from 0.56 to $3.2 \mu \mathrm{L} / \mathrm{mL}$ induced significantly higher nitric oxide production compared to unstimulated cells $\left(\mathrm{E}_{\max }=34.7 \mu \mathrm{M}\right.$ nitrite at $\left.1.0 \mu \mathrm{L} / \mathrm{mL}\right)$ (Figure 2a). At doses > I.0 $\mu \mathrm{L} / \mathrm{mL}$, nitric oxide production gradually decreased, which can be explained by the observed cytotoxicity of the vaccine at high concentrations, as assessed by 7-AAD viability staining (Supplementary Figure SI).

Gene expression of iNOS was measured to investigate the activation of the nitric oxide production pathway over time as the accumulation of nitric oxide cannot be detected at early time points (not shown). Expression of iNOS was strongly upregulated between 4 and $6 \mathrm{~h}$ after exposure to $1.0 \mu \mathrm{L} / \mathrm{mL}$ octavalent vaccine, reaching a 59-fold increase at $\mathrm{t}=6 \mathrm{~h}$ in comparison to $\mathrm{t}=0 \mathrm{~h}$ (Figure $\mathbf{2 b}$ ). The maximum increase in iNOS expression was observed at $\mathrm{t}=24 \mathrm{~h}$ ( $3 \mathrm{I} \mathrm{I}$-fold) and expression remained high at least up to $48 \mathrm{~h}$ (289-fold), which was the last time point assessed.

(a)

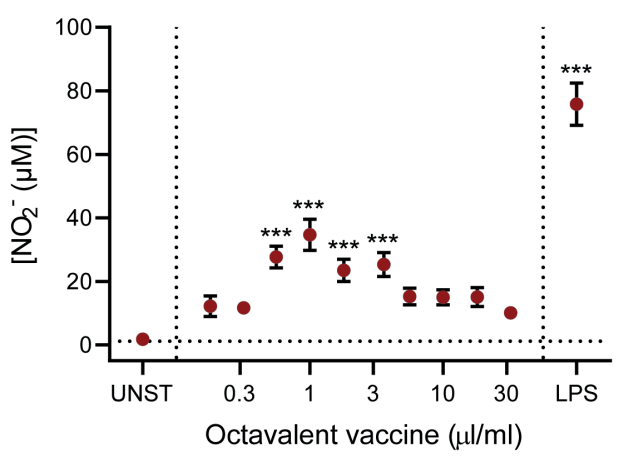

(b)

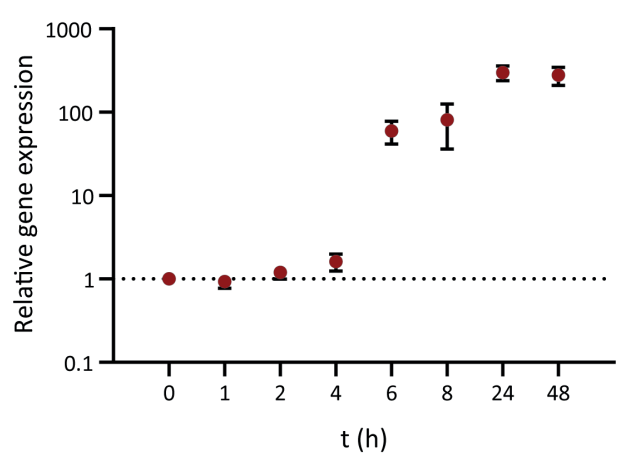

Figure 2. Nitric oxide was produced upon exposure to octavalent vaccine (IBV + NDV + EDSV $+5 x$ Av. paragallinarum) for $48 \mathrm{~h}$. (a) HDIl cells were exposed to titrated concentrations of the octavalent vaccine. Moreover, unstimulated HDII cells (UNST) and HDII cells stimulated with $300 \mathrm{ng} / \mathrm{ml}$ LPS are shown as negative and positive controls. The data comprises three independent technical replicates performed in triplicate. The error bars represent the SEM. A Kruskal-Wallis test combined with Dunn's multiple comparisons test was used to test for significant induction of nitric oxide production upon stimulation. $* * * p<0.00$ I. (b) RT-qPCR was performed on HDI I cell samples stimulated with I $\mu \mathrm{L} / \mathrm{mL}$ octavalent vaccine and harvested at the given time points between 0 and 48 h. iNOS expression is shown relative to $t=0 \mathrm{~h}$ and expressed as $2^{-\Delta \Delta \mathrm{Ct}}$ values as calculated using the Livak method and GAPDH and $28 \mathrm{~S}$ as a reference gene. The experiment was performed in triplicate. The error bars represent the SEM. 


\section{Stimulation with the octavalent vaccine results in enhanced gene expression of cytokines and chemokines}

Cytokine gene expression by vaccine-stimulated HDI I cells was followed over time. As early as I $\mathrm{h}$ after exposure to the vaccine, HDII cells upregulated gene expression levels of the pro-inflammatory cytokine IL-I $\beta$ (4.3-fold) and IL-8-like chemokines CXCLil (2.9-fold) and CXCLi2 (3.3-fold) (Figure 3a-c), which increased up to $8 \mathrm{~h}$ after exposure to respectively 584-, 99.9-, and 225-fold. Expression of IL-I $\beta$ subsequently decreased to 30.0-fold at $24 \mathrm{~h}$ and 9.3-fold at $48 \mathrm{~h}$ as compared to $\mathrm{t}=0 \mathrm{~h}$ (Figure 3a), whereas expression of CXCLil and CXCLi2 remained high up to $48 \mathrm{~h}$ (Figure $3 \mathbf{b}, \mathbf{c}$ ). HDI I cells showed a modest upregulation of the pro-inflammatory cytokine TNF after exposure to the octavalent vaccine, reaching its peak at $\mathrm{t}=8 \mathrm{~h}$ with a 4.0 -fold increase in expression (Figure 3d). In contrast, stimulation with the vaccine did not induce gene expression of IL- 6 and IFN- $\alpha$ at any of the time points studied (Figure 3e,f). Expression of the anti-inflammatory cytokine IL- I0 was elevated after

(a)

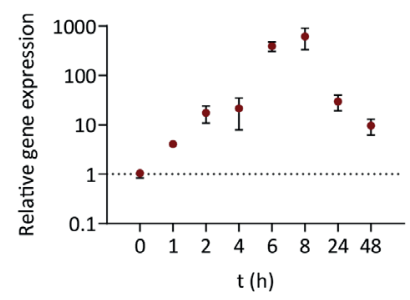

(d)

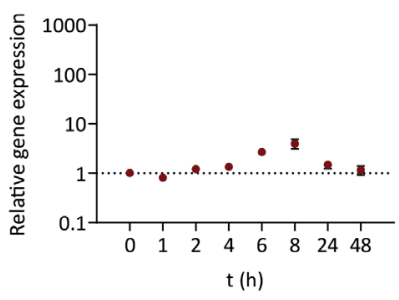

(g)

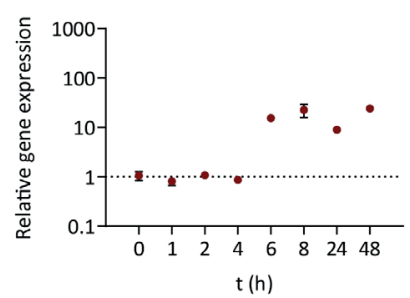

(b)

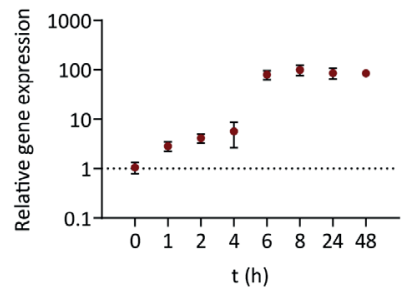

(e)

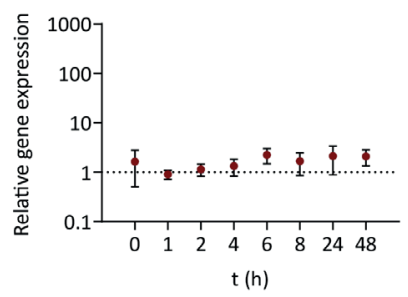

(h).

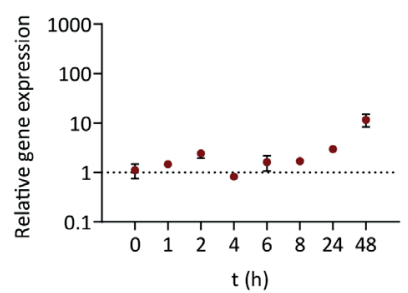

(c)

CXCLi2

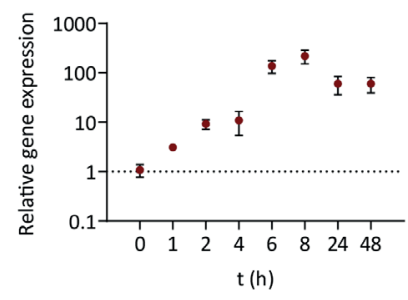

(f)

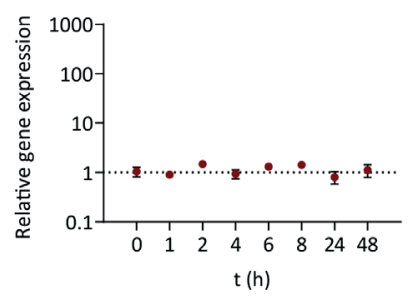

(i)

IFN- $\gamma$

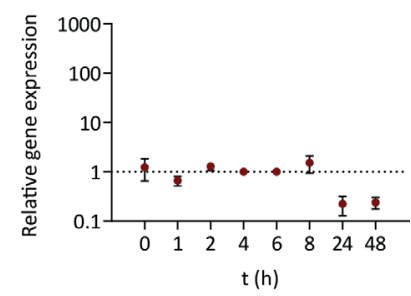

Figure 3. The octavalent vaccine induced the expression of pro-inflammatory cytokines at an early time point, followed by expression of the anti-inflammatory cytokine IL- I0 and finally IL-I 2 p 40 . HDI I cells were stimulated with $\mathrm{I} \mu \mathrm{L} / \mathrm{mL}$ octavalent vaccine and harvested at the indicated time points between 0 and 48 h. RT-qPCR was performed for the cytokines IL-I $\beta$ (a), CXCLil (b), CXCLi2 (c), TNF (d), IL-6 (e), IFN- $\alpha$ (f), IL-I0 (g), IL-I 2p40 (h) and IFN- $\gamma$ (i). The relative gene expression levels were normalized against $\mathrm{t}=0 \mathrm{~h}$ and expressed as $2^{-\Delta \Delta \mathrm{Ct}}$ values as calculated using the Livak method and both GAPDH and $28 \mathrm{~S}$ as reference genes. The experiment was performed in triplicate. The error bars represent the SEM. 
$6 \mathrm{~h}$ to 19.5 -fold and remained at that level up to $48 \mathrm{~h}$ (Figure $3 \mathrm{~g}$ ). Expression of the $\mathrm{T}_{\mathrm{H}} \mathrm{I}$ or 17-inducing cytokine IL-I $2 p 40$, depending on its heterodimerization with respectively IL-I2p35 [36] or IL-23pI9 [37], was increased maximally II.7-fold at $\mathrm{t}=48 \mathrm{~h}$ (Figure 3h). Expression of $\mathrm{T}_{\mathrm{H}} \mathrm{I}$-inducing cytokine IFN- $\gamma$ was found slightly decreased at 24 and 48 $\mathrm{h}$ (4.50- and 4.I4-fold decrease respectively) compared to $\mathrm{t}=0 \mathrm{~h}$ (Figure $3 \mathrm{i}$ ). The $\mathrm{T}_{\mathrm{H}} 2$ inducing cytokine IL-4 and $\mathrm{T}_{\mathrm{H}} \mathrm{I}$-inducing cytokine IL-I 2p35 were not detected (not shown). Taken together, HDII cells showed increased expression of pro-inflammatory cytokines $\mathrm{IL}-\mathrm{I} \beta$ and TNF, and chemokines CXCLil and CXCLi2 within $8 \mathrm{~h}$ after exposure to the octavalent vaccine, which was followed by the induction of the anti-inflammatory cytokine $\mathrm{IL}-\mathrm{I} 0$ and the $\mathrm{T}_{\mathrm{H}} \mathrm{I} / \mathrm{T}_{\mathrm{H}} \mathrm{I}$-inducing cytokine IL- $12 \mathrm{p} 40$.

\section{Av. paragallinarum antigens contribute to the stimulatory capacity of the octavalent vaccine}

To investigate whether Av. paragallinarum antigens were able to stimulate HDII cells, the responses induced by the octavalent vaccine were compared to responses induced by a trivalent vaccine containing the same viral antigens but without bacterial Av. paragallinarum antigens. In contrast to the octavalent vaccine, the trivalent vaccine did not lead to nitric oxide production by HDII cells (Figure $\mathbf{4 a}$ ). These results strongly suggested that the Av. paragallinarum antigens were responsible for the nitric oxide production by HDII cells. Next, antigens were extracted from the emulsion vaccines using isopropyl myristate. As shown in Figure $\mathbf{4 b}$, the antigens extracted from the octavalent vaccine induced more nitric oxide production than the octavalent vaccine itself $(50.2 \mu \mathrm{M}$ vs. $6.7 \mu \mathrm{M}$ nitrite at $0.3 \mu \mathrm{L} / \mathrm{mL}$, respectively). In contrast, the antigens extracted from the trivalent vaccine did not induce nitric oxide production. Compared to the complete octavalent vaccine with an $\mathrm{LC}_{50}$ of 18 $\mu \mathrm{L} / \mathrm{mL}$, the extracted antigens were more cytotoxic for HDI I cells with a $\mathrm{LC}_{50}$ of $0.26 \mu \mathrm{L} /$ $\mathrm{mL}$ (Supplementary Figure SI). To determine whether these cytotoxic effects were due to the bacterial antigens or other vaccine constituents, the bacteria were recovered from the antigenic fraction of the octavalent vaccine by centrifugation at high speed, followed by extensive washing in PBS, and resuspended in PBS according to the original volume of antigenic fraction (Supplementary Figure S2). The bacteria stimulated HDII cells to produce high concentrations of nitric oxide $\left(E_{\max }=57.1 \mu M\right.$ nitrite at $\left.1.0 \mu \mathrm{L} / \mathrm{mL}\right)$ over a wide range of concentrations (Figure 4c), whereas the cytotoxicity of the purified bacteria $\left(\mathrm{LC}_{50}=4.2 \mu \mathrm{L} / \mathrm{mL}\right)$ was reduced compared to the antigenic fraction $\left(\mathrm{LC}_{50}=0.26 \mu \mathrm{L} / \mathrm{mL}\right)$ (Supplementary Figure SI). The potency of the purified bacteria to induce nitric oxide production, as expressed by an $\mathrm{EC}_{50}$-value, was found to be $=0.276 \mu \mathrm{L} / \mathrm{mL}$.

Next, the gene expression levels of iNOS and cytokines were determined $8 \mathrm{~h}$ after stimulation with either the tri- or octavalent vaccine. Exposure to the octavalent vaccine led to significantly increased expression of iNOS (36.4-fold), IL-I $\beta$ (7I.4-fold), TNF (I.7-fold), CXCLil (46.9-fold), CXCLi2 (55.7-fold) (Figure 4e-i), and IL-I0 (40-Ct: 4.0 for octavalent vaccine vs. I.I for unstimulated; Supplementary Figure S3). In contrast, the trivalent vaccine did not induce the expression of iNOS or any of the cytokines. Expression IFN- $\alpha$ was slightly decreased after stimulation with either the tri- (2.I-fold decrease) or octavalent (2.0-fold decrease) vaccine (Figure 4j). 
(a)

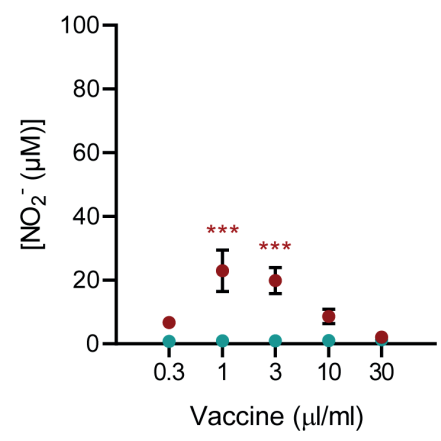

(b)

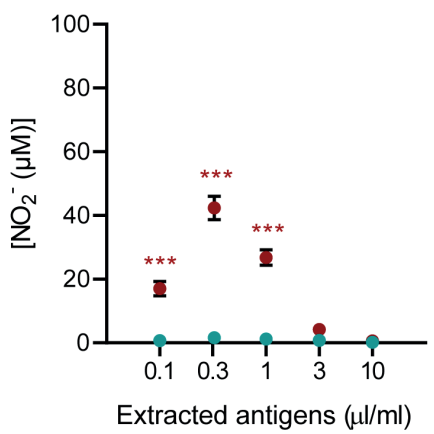

(c)

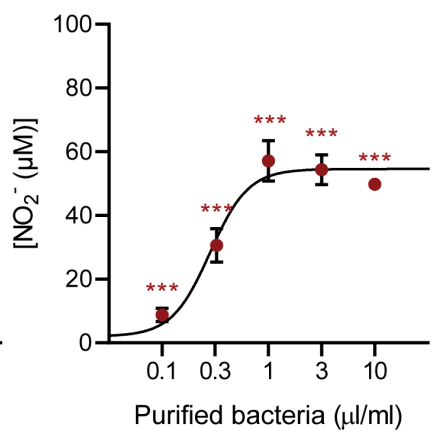

- Trivalent vaccine

- Octavalent vaccine

(d)

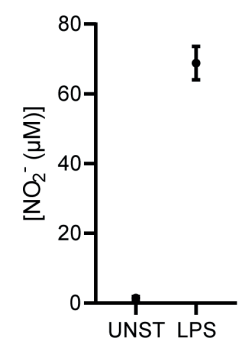

(e)

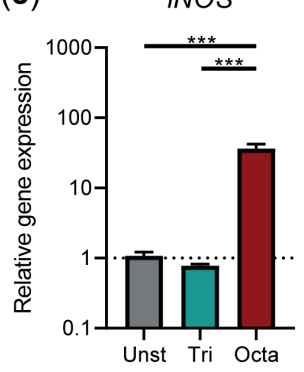

(h)

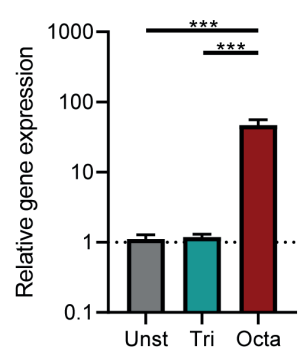

(f)

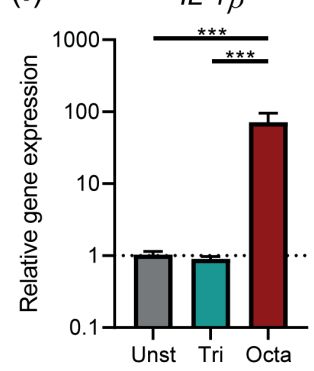

(i)

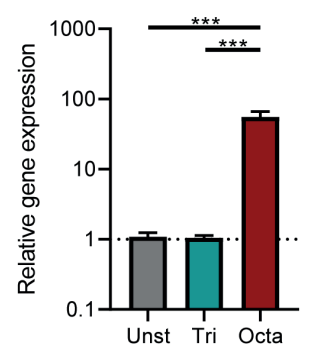

(g)

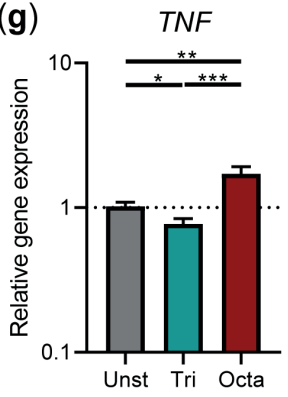

(j)

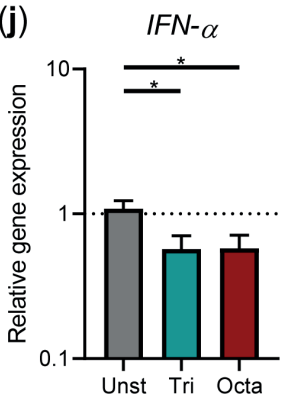

Figure 4. A trivalent vaccine without $A v$. paragallinarum antigens did not induce nitric oxide production or expression of pro-inflammatory cytokines. (a) Nitric oxide production was determined for HDI I cells exposed to titrated doses of trivalent (IBV + NDV + EDSV) and octavalent vaccine (IBV + NDV + EDSV + 5x Av. paragallinarum) for $48 \mathrm{~h}$. (b) Antigenic fractions were extracted from the triand octavalent vaccines using isopropyl myristate and given to HDI I cells to determine nitric oxide production after $48 \mathrm{~h}$. (c) The bacterial pellet was purified from the antigenic fraction of the octavalent vaccine and given to HDII cells to determine nitric oxide production after $48 \mathrm{~h}$. A four-parameter logistic curve could be calculated and was plotted. (d) The controls of the nitric oxide production assay included unstimulated HDI I cells (UNST) and HDI I cells stimulated with $300 \mathrm{ng} / \mathrm{ml}$ LPS. (e-j) Expression levels of iNOS (e), IL-I $\beta$ (f), TNF (g), CXCLil (h), CXCLi2 (i), and IFN- $\alpha$ (j) by HDII cells were determined $8 \mathrm{~h}$ after stimulation with $1.0 \mu \mathrm{L} / \mathrm{mL}$ tri- or octavalent vaccine. The values are expressed as $2^{-\Delta \Delta C t}$ values as calculated using the Livak method and both GAPDH and $28 \mathrm{~S}$ as reference genes. All figures show three independent technical replicates. The error bars represent the SEM. A Kruskal-Wallis test combined with Dunn's multiple comparisons test was used to test for statistical significance of the data. $* p<0.05, * * p<0.01$, ${ }^{* * *} p<0.001$. The relative gene expression data were log-transformed prior to the statistical analysis to generate normally distributed data. 


\section{Activation of HD I I cells by the octavalent vaccine largely depends on Av. Paragallinarum antigen-associated LPS}

Finally, the antigenic fraction of the octavalent vaccine was pre-incubated with the LPSbinding antibiotic polymyxin B to determine the contribution of LPS, embedded in the cell wall of $A v$. paragallinarum bacteria, to HDII cell activation. First, E. coli LPS (Figure 5a) and CpG ODN2006 (Figure 5b), acting as positive and negative controls, were both preincubated with polymyxin B for I h and subsequently administered to HDI I cell cultures. Polymyxin B significantly inhibited LPS-induced nitric oxide production (36.5\% reduction), whereas $C_{p} G$-induced nitric oxide production remained unchanged. Next, the antigenic fraction of the octavalent vaccine was preincubated with polymyxin $B$ and administered to the cells (Figure 5c), which resulted in a significant reduction in nitric oxide production of $65.7 \%$ as compared to exposure to the antigenic fraction alone. Moreover, the treatment of the antigenic fraction of the octavalent vaccine with polymyxin $B$ resulted in a significant reduction in the expression of iNOS (67.6\% reduction; Figure $5 \mathbf{d})$, IL- I $\beta$ ( $80.0 \%$ reduction; Figure 5e), and CXCLil (58.9\% reduction; Figure 5f). The expression levels of CXCLi2 ( $35.1 \%$ reduction; Figure $\mathbf{5 g}$ ) and IL- 10 (43.5\% reduction; Figure $\mathbf{5 h}$ ) tended to be inhibited by pre-incubation with polymyxin B, but this was not statistically significant. Taken together, these experiments suggest that LPS present in Av. paragallinarum antigens significantly contributed to the activation of HDI I cells upon exposure to the octavalent vaccine.

\section{Discussion}

In this explorative study, we aimed to investigate the capacity of an octavalent vaccine containing inactivated IBV, NDV, EDSV, and five serovars of Av. paragallinarum to activate innate immune cells, in view of identifying potential immune parameters that could be used in the future for in vitro vaccine $\mathrm{QC}$ testing of inactivated Av. paragallinarum. The vaccine was found to activate the chicken macrophage-like cell line HDII shortly after stimulation, resulting in the expression of the nitric oxide-producing enzyme iNOS and the pro-inflammatory cytokines IL-I $\beta$, TNF, CXCLil, and CXCLi2. From $6 \mathrm{~h}$ after stimulation, HDI I cells expressed the anti-inflammatory cytokine IL- I0, which inhibits TLR signaling [38] and may be responsible for the subsequent return of IL-I $\beta$ and TNF expression to baseline levels. In contrast, HDII cells were not activated by a trivalent vaccine containing the same inactivated viral antigens but without inactivated $A v$. paragallinarum antigens, indicating that bacterial PAMPs were important for the evoked immune response. Furthermore, pre-treating the extracted antigens with the LPS-neutralizing antibiotic polymyxin B [32] largely averted nitric oxide production and cytokine expression by HDI I cells, showing that bacterial LPS was an important immunostimulatory factor of the octavalent vaccine. Hence, stimulation of HDII cells by the octavalent vaccine must at least partly be dependent on TLR4, the designated PRR for LPS [39]. This is supported by an in vivo study, showing upregulation of the TLR4 signaling pathway in the nasal tissues of chickens infected with Av. paragallinarum [40].

The differences in macrophage activation by the octa- and trivalent vaccine were striking and may be explained by the presence or absence of bacterial PAMPs from Av. paragallinarum acting as an endogenous adjuvant of the octavalent vaccine. This is in agreement with in vivo data demonstrating that chickens developed strong granulomatous reactions involving macrophages at injection sites of inactivated bacterial Av. paragallinarum or Mycoplasma 


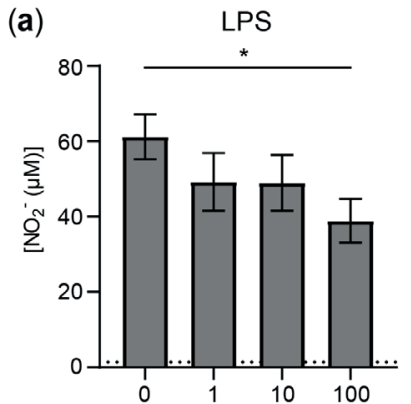

[Polymyxin B $(\mu \mathrm{g} / \mathrm{ml})$ ] (b)

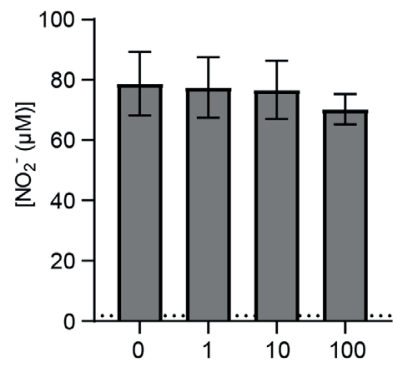

[Polymyxin B $(\mu \mathrm{g} / \mathrm{ml})$ ]

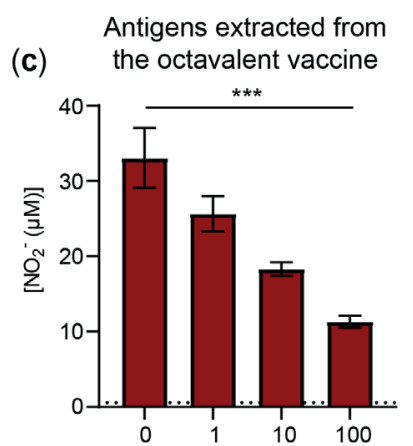

[Polymyxin B $(\mu \mathrm{g} / \mathrm{ml})]$

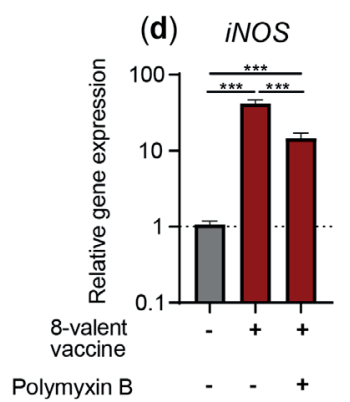

(e)

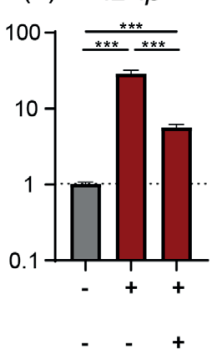

(f) CXCLi1

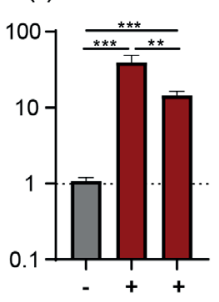

(g) $\mathrm{CXCLi2}$

(h) $\quad I L-10$
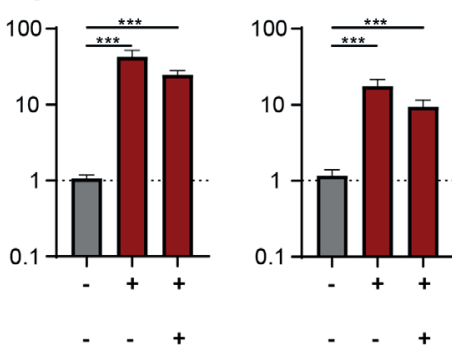

Figure 5. Nitric oxide production upon exposure to the antigenic fraction of the octavalent vaccine could be inhibited by the LPS-binding antibiotic polymyxin B. (a-c) HDII cells were pre-incubated in cell culture media without or with I, 10 , or $100 \mu \mathrm{g} / \mathrm{ml}$ polymyxin B for I h at $37^{\circ} \mathrm{C}$ and subsequently exposed to cell culture media containing $300 \mathrm{ng} / \mathrm{ml}$ LPS (a), $300 \mathrm{ng} / \mathrm{ml}$ CpG (b) or $0.5 \mu \mathrm{L} / \mathrm{mL}$ antigens extracted from the octavalent vaccine (c) for $48 \mathrm{~h}$. Unstimulated controls are represented by the dotted lines. The first bar of panel a is the result of HDII stimulated with $300 \mathrm{ng} / \mathrm{ml}$ without polymyxin B and thus represents the positive control. (d-h) Expression levels of iNOS (d), IL-I $\beta$ (e), CXCLil (f), CXCLi2 (g), and IL-IO (h) by HDI I cells were determined $8 \mathrm{~h}$ after stimulation with I $\mu \mathrm{L} / \mathrm{mL}$ octavalent vaccine and, when indicated, I h pre-incubation with $100 \mu \mathrm{g} / \mathrm{ml}$ polymyxin $\mathrm{B}$. The values are expressed as $2^{-\Delta \Delta C t}$ values as calculated using the Livak method and both GAPDH and $28 \mathrm{~S}$ as reference genes. The data comprises three independent technical replicates performed in triplicate. Unstimulated controls are represented by the dotted lines. The error bars represent the SEM. A Kruskal-Wallis test combined with Dunn's multiple comparisons test was used to test for statistical significance of the data. ${ }^{*} p<0.05$, ** $p<0.01$, *** $p<0.001$. The relative gene expression data were log-transformed prior to the statistical analysis to create normally distributed data.

gallisepticum vaccines, but not at injection sites of inactivated viral NDV, IBV, avian reovirus, or infectious bursal disease virus vaccines [4I,42,43]. The presence of bacterial antigens containing PAMPs in the multivalent vaccine most likely also boosts the immune responses against the antigens of viral origin, as demonstrated for an experimental vaccine against both inactivated viral influenza $A$ and Streptococcus pneumonia in mice [15].

The role of nitric oxide production and cytokines to the induction of vaccine-mediated protection against Av. paragallinarum in chickens is still unknown. A genome-wide association study in chickens has shown that there is an association between serological responses and small nucleotide polymorphism within and surrounding the gene encoding for iNOS, suggesting that iNOS may also be important in chickens during vaccination [44]. Studies 
with iNOS, IL-I7, IL-4, and IFN- $\gamma$-deficient mice have demonstrated the importance of nitric oxide [45] and cytokine production [ I7] for effective vaccination against Bordetella pertussis, also being a Gram-negative bacterium, and Trypanosoma cruzi [46]. Studies in humans mice and humans have more specifically demonstrated the importance of the cytokine-mediated induction of a mixed $\mathrm{T}_{\mathrm{H}} \mathrm{I} / \mathrm{T}_{\mathrm{H}} \mathrm{I} / \mathrm{response}$ for effective vaccination against Bordetella pertussis $[17, \mid 8]$. Similarly, a study in chickens has demonstrated that a nasal challenge with Pasteurella multocida, a member of the same bacterial family as $A v$. paragallinarum, results in a mixed $\mathrm{T}_{\mathrm{H}} \mathrm{I} / \mathrm{T}_{\mathrm{H}} \mathrm{I} \mathrm{T}$ response [47]. Similar to mice, a strong pro-inflammatory cytokine response is likely to be important for effective vaccination in chickens, as demonstrated for a flagellinadjuvanted vaccine for Pasteurella multocida [48]. A nasal challenge with Av. paragallinarum in chickens was previously shown to result in the increased local gene expression of IL-6, which may contribute to a $T_{H} I 7$ adaptive immune response in mammals [49], whereas no changes were observed for the $\mathrm{T}_{\mathrm{H}} \mathrm{I}$-inducing cytokine IL-I 2p35 [50]. In this in vitro study, we found increased gene expression of the $T_{H} I / T_{H} I 7$-inducing cytokine IL-I2p40. We did unexpectedly not observe increased IL-6 expression by HDI I cells after stimulation with the octavalent vaccine, despite previous studies showing that bacterial stimulation induces IL-6 expression in HDI I cells [24]. The $\mathrm{T}_{H} \mathrm{I}$-incuding cytokine IL-I2p35 and the $\mathrm{T}_{\mathrm{H}} 2$-incuding cytokine IL-4 remained undetectable in HDI I cells at any of the time points after stimulation with the octavalent vaccine. The type of adaptive immune response that is triggered by $A v$. paragallinarum during natural infection or vaccination remains interesting for further investigation.

Since the potency of a vaccine does not depend solely on antigen quantity but also on its immunostimulatory capacity, the preservation and consistency of LPS and other PAMPs in inactivated $A v$. paragallinarum vaccines is likely to be important for vaccination [5I,52,53,54]. The immunostimulatory capacity of $A v$. paragallinarum bacteria incorporated in the octavalent vaccine could be evaluated using the nitric oxide production assay and was expressed as the half-maximum effective concentration $\left(\mathrm{EC}_{50}\right)$-value, which here is the vaccine concentration that gives half-maximal nitric oxide production. This opens up the possibility to use the nitric production assay, in addition to antigen quantification methods, to test vaccines containing inactivated Av. paragallinarum (and potentially other Gram-negative bacteria) for potency without the use of animals. The bacterial antigens of the octavalent vaccine were extracted from the w/o emulsion with isopropyl myristate, followed by centrifugation steps, to enable the quantification of the immunostimulatory capacity of the vaccine. Testing extracted antigens, rather than the native vaccine formulation, is a strategy that is also used to test inactivated NDV vaccines for potency by an ELISA and has been implemented in the European Pharmacopoeia monograph 0870 [7,9]. Future studies should address the suitability of the nitric oxide assay to discriminate between vaccine batches of different potency, including non-conforming batches, in accordance with the consistency approach [I]. Furthermore, in our study, we observed that high cell passage numbers of the HDII cell line may slightly affect the sensitivity of the nitric oxide production assay (not shown), which needs to be addressed in assay validation studies. The inclusion of reference standards for normalization might be required to improve precision. Finally, the performance of the assay as a QC test should be compared to the animal-based vaccination-challenge test that is currently in place as the gold standard for infectious coryza vaccines [13]. 


\section{Conclusions}

This explorative study aimed to investigate the immunostimulatory capacity of an inactivated octavalent vaccine for IBV, NDV, EDSV, and Av. paragallinarum and to identify immune parameters that could potentially influence vaccine potency. We have found that this inactivated octavalent poultry vaccine activates the chicken macrophage-like cell line HDI I due to the presence of LPS associated with Av. paragallinarum antigens, which resulted in the production of nitric oxide and expression of the pro-inflammatory cytokine IL-I $\beta$ and chicken IL-8-like chemokine CXCLiI. In contrast, a trivalent vaccine containing inactivated IBV, NDV, and EDSV viral antigens, which are also present in the octavalent vaccine, did not induce nitric oxide production or cytokine expression by HDI I cells, further demonstrating that the responses measured in the assays were specific for the Av. paragallinarum antigens of the octavalent vaccine. Furthermore, the nitric oxide production assay was shown to be potentially useful as an in vitro potency test for inactivated poultry vaccines against $A v$. paragallinarum using the $\mathrm{EC}_{50}$ of the purified bacteria as a readout for potency. Therefore, this study may contribute to the replacement of current animal-based vaccine $Q C$ tests and improve animal welfare.

\section{Acknowledgements}

The authors thank Sabine Roersma for performing the initial experiments that led to this study. Furthermore, the authors thank the Veterinary Microbiological Diagnostic Centre of the Faculty of Veterinary Medicine, Utrecht University, for performing the Gram staining of our samples. The microscopy images have been acquired at the Center of Cellular Imaging, Faculty of Veterinary Medicine, Utrecht University. Flow cytometry was performed at the Flow Cytometry and Cell Sorting Facility, Faculty of Veterinary Medicine, Utrecht University.

\section{Funding}

This research was funded by the Innovative Medicines Initiative 2 Joint Undertaking under grant agreement No I I 5924 (VAC2VAC). This Joint Undertaking receives support from the European Union's Horizon 2020 research and innovation program and EFPIA. The contents of this article represent the authors' scientific conclusions and neither IMI nor the European Union, EFPIA, or any Associated Partners are responsible for any use that may be made of the information contained therein. 


\section{Supplementary Materials}

(a)

HD11 culture

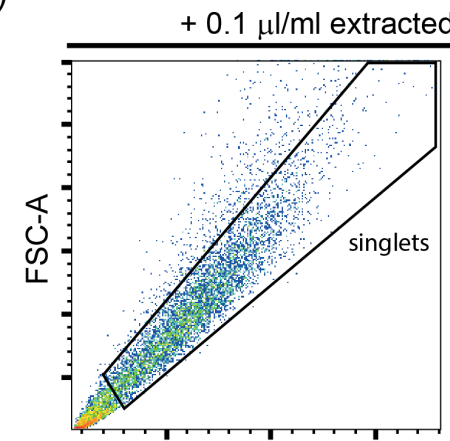

FSC-H

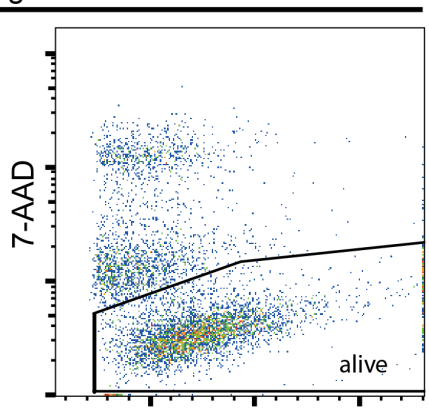

FSC

(b)

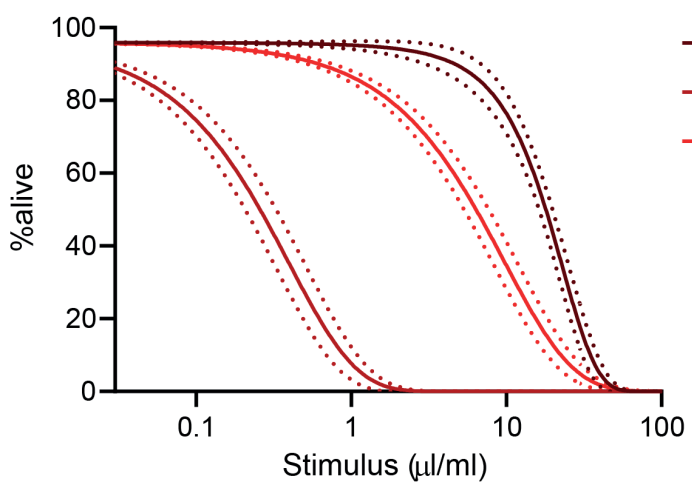

- Octavalent vaccine

- Extracted antigens

- Purified bacteria

Supplementary Figure SI. Cytotoxicity of the octavalent vaccine, extracted antigens and purified Av. paragallinarum bacteria. (a) The HDI I cells were stained with 7-aminoactinomycin $D$ to determine the percentage of viable cells by flow cytometry. The cells were first gated for single cells, also excluding debris. Next, cells negative for 7-AAD were considered to be alive. (b) HDII cells were stimulated with graded doses of octavalent vaccine $(0.3-30 \mu \mathrm{l} / \mathrm{ml})$, the antigens extracted from the octavalent vaccine $(0.1-10 \mu \mathrm{l} / \mathrm{ml})$ and purified Av. paragallinarum bacteria $(0.1-10 \mu \mathrm{l} / \mathrm{ml})$ for $48 \mathrm{~h}$. The graph was created by plotting survival curves with confidence intervals. 
(a) Extracted antigens from trivalent vaccine

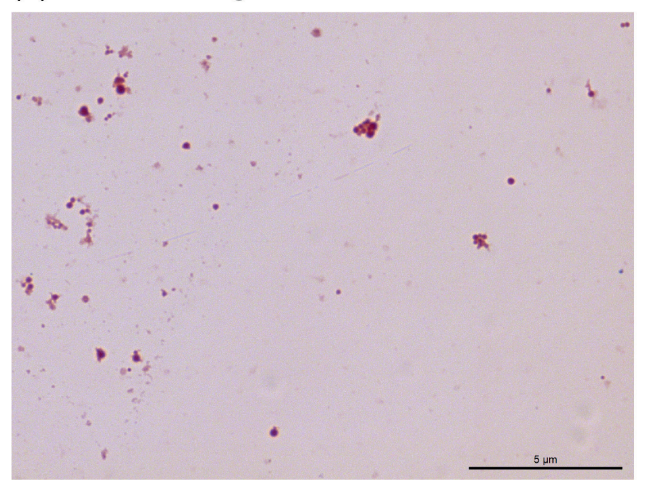

(b) Extracted antigens from octavalent vaccine

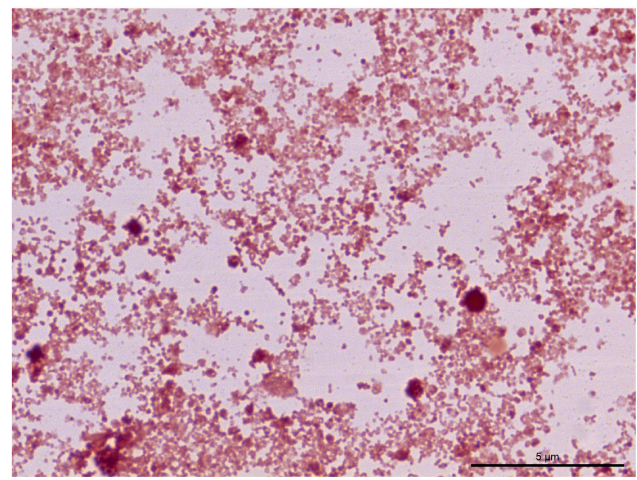

Supplementary Figure S2. Gram staining of extracted antigens from the tri- (a) and octavalent (b) vaccines. Av. paragallinarum bacteria were purified from the octavalent vaccine as described in the Materials and Methods. As a control, the antigens from the trivalent vaccines were purified using the same procedure. The images were taken at 1000x magnification using an Olympus BX60 microscope and immersion oil. The scale bars in black represent $5 \mu \mathrm{m}$.

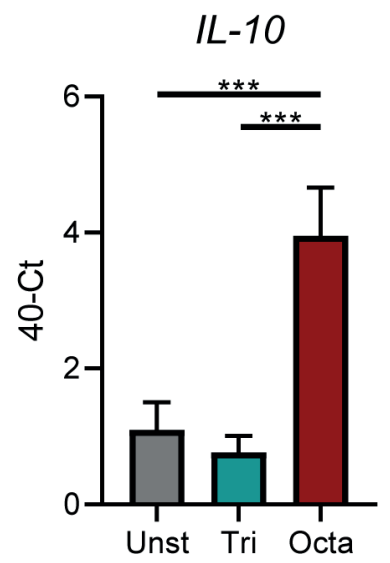

Supplementary Figure S3. The octavalent vaccine induced gene expression of IL-I0, whereas a trivalent vaccine without Av. paragallinarum antigens did not. Gene expression levels of IL- I0 by HDI I cells were determined $8 \mathrm{~h}$ after stimulation with $1.0 \mu \mathrm{l} / \mathrm{ml}$ tri- or octavalent vaccine and are expressed as $40-\mathrm{Ct}$ values, as described by Eldaghayes et al. [34]. The figure shows three independent technical replicates. The error bars represent the SEM. A Kruskal-Wallis test combined with Dunn's multiple comparisons test was used to test for statistical significance of the data. $* * * p<0.001$. 


\section{References}

I. Hendriksen C, Arciniega JL, Bruckner L, Chevalier M, Coppens E, Descamps J, et al. The consistency approach for the quality control of vaccines. Biologicals (2008) 36(I):73-77. doi: 10.1016/j.biologicals.2007.05.002

2. Hendriksen CF. Replacement, reduction and refinement alternatives to animal use in vaccine potency measurement. Expert Rev Vaccines (2009) 8(3):3 I3-322. doi:I0.1586/I4760584.8.3.3 I3

3. Bruysters MWP, Schiffelers MJ, Hoonakker M, Jungbaeck C, Ragan I, Rommel E, et al. Drivers and barriers in the consistency approach for vaccine batch release testing: Report of an international workshop. In: Biologicals. Vol 48. Academic Press; 2017:I-5. doi:I0.1016/j.biologicals.2017.06.006

4. Blackall PJ, Soriano E V. Infectious coryza and related bacterial infections. In: Saif YM, Fadly AM, Glisson JR, McDougald LR, Nolan LK, Swayne DE, eds. Diseases of Poultry. 12th ed. Blackwell Publishing; 2008:789-803.

5. Page LA. Haemophilus infections in chickens. I. Characteristics of 12 Haemophilus isolates recovered from diseased chickens. Am J Vet Res (1962) 23:85-95.

6. Otsuki K, Iritani Y. Preparation and Immunological Response to a New Mixed Vaccine Composed of Inactivated Newcastle Disease Virus, Inactivated Infectious Bronchitis Virus, and Inactivated Hemophilus gallinarum. Avian Dis (1974) I8(3):297. doi: I0.2307/I589097

7. Maas PA, de Winter MPM, Venema S, Oei HL, Claassen IJTM. Antigen quantification as in vitro alternative for potency testing of inactivated viral poultry vaccines. Vet $Q 2000$. doi: $10.1080 / 01$ 652176.2000 .9695063

8. Claassen I, Maas R, Oei H, Daas A, Milne C. Validation study to evaluate the reproducibility of a candidate in vitro potency assay of newcastle disease vaccines and to establish the suitability of a candidate biological reference preparation. Pharmeuropa bio (2004) 2004(I): I-I5.

9. EDQM. Monograph 870. Newcastle Disease Vaccine (Inactivated). In: European Pharmacopoeia. 9th ed. Strasbourgh, France: European Department for the Quality of Medicines; 2017:10801082.

10. EDQM. Monograph 0959. Avian Infectious Bronchitis Vaccine (Inactivated). In: European Pharmacopoeia. 9th ed. Strasbourgh, France: European Department for the Quality of Medicines; 2017:1007-1008.

II. EDQM. Monograph 1202. Egg Drop Syndrome '76 Vaccine (Inactivated). In: European Pharmacopoeia. 9th ed. Strasbourgh, France: European Department for the Quality of Medicines; 2017:1048-1049.

12. EDQM. Vaccines for veterinary use. In: European Pharmacopoeia. 9.5th. Strasbourgh, France: European Department for the Quality of Medicines; 2018:5574-5579.

13. García A, Romo F, Ortiz AM, Blackall PJ. The vaccination-challenge trial: the gold standard test to evaluate the protective efficacy of infectious coryza vaccines. Avian Pathol (2008) 37(2): I83- 186. doi: I0.1080/0307945080|92994|

14. Kleinnijenhuis J, Quintin J, Preijers F, Joosten LAB, Ifrim DC, Saeed S, et al. Bacille CalmetteGuérin induces NOD2-dependent nonspecific protection from reinfection via epigenetic reprogramming of monocytes. Proc Natl Acad Sci U S A (20I2) 109(43): I7537-I7542. doi: 10.1073/pnas. 1202870109

15. David SC, Norton T, Tyllis T, Wilson JJ, Singleton E V., Laan Z, et al. Direct interaction of wholeinactivated influenza $A$ and pneumococcal vaccines enhances influenza-specific immunity. Nat Microbiol (2019) 4(8):I316-1327. doi:I0.1038/s4I564-0I9-0443-4

16. Sander LE, Davis MJ, Boekschoten M V., Amsen D, Dascher CC, Ryffel B, et al. Detection of prokaryotic mRNA signifies microbial viability and promotes immunity. Nature (20II) 474(735I):385-392. doi:10.1038/naturel 0072

17. Ross PJ, Sutton CE, Higgins S, Allen AC, Walsh K, Misiak A, et al. Relative Contribution of $T_{H}$ I and $T_{H} 17$ Cells in Adaptive Immunity to Bordetella pertussis: Towards the Rational Design of an Improved Acellular Pertussis Vaccine. PLoS Pathog (20I3) 9(4). doi:I 0. I37 I/journal.ppat. 003264 
18. Higgs R, Higgins SC, Ross PJ, Mills KHG. Immunity to the respiratory pathogen Bordetella pertussis. Mucosal Immunol (20I2) 5(5):485-500. doi:I0.1038/mi.20I2.54

19. Tsuji S, Matsumoto M, Takeuchi O, Akira S, Azuma I, Hayashi A, et al. Maturation of human dendritic cells by cell wall skeleton of Mycobacterium boris bacillus Calmette-Guérin: Involvement of tolllike receptors. Infect Immun (2000) 68(I2):6883-6890. doi: I 0. I I 28/IAI.68. I 2.6883-6890.2000

20. Desbien AL, Dubois Cauwelaert N, Reed SJ, Bailor HR, Liang H, Carter D, et al. IL-I8 and Subcapsular Lymph Node Macrophages are Essential for Enhanced B Cell Responses with TLR4 Agonist Adjuvants. J Immunol (20 I6) I97(I I):435I -4359. doi: I0.4049/jimmunol. 1600993

21. Beug H, von Kirchbach A, Döderlein G, Conscience JF, Graf T. Chicken hematopoietic cells transformed by seven strains of defective avian leukemia viruses display three distinct phenotypes of differentiation. Cell (1979) 18(2):375-390. doi:10.1016/0092-8674(79)90057-6

22. Leutz A, Beug H, Graf T. Purification and characterization of $\mathrm{CMGF}$, a novel chicken myelomonocytic growth factor. EMBO J (I984) 3(I3):3 I9I-3 I 97. doi:I0. I002/j. I 460-2075.1984.tb02278.x

23. Iqbal M, Philbin VJ, Smith AL. Expression patterns of chicken Toll-like receptor mRNA in tissues, immune cell subsets and cell lines. Vet Immunol Immunopathol (2005) I04(I-2): I I7-I 27. doi:I0.1016/J.VETIMM.2004.II.003

24. Peng L, Matthijs MGR, Haagsman HP, Veldhuizen EJA. Avian pathogenic Escherichia coli-induced activation of chicken macrophage HDI I cells. Dev Comp Immunol (2018) 87:75-83. doi: 10. I0I6/j. dci.2018.05.019

25. Wisner ALS, Potter AA, Kö Ster W. Effect of the Salmonella Pathogenicity Island 2 Type III Secretion System on Salmonella Survival in Activated Chicken Macrophage-Like HDII Cells. 20II. doi:I0.I37I/journal.pone.0029787

26. Biggelaar RHGA van den, Eden W van, Rutten VPMG, Jansen CA. Nitric Oxide Production and Fc Receptor-Mediated Phagocytosis as Functional Readouts of Macrophage Activity upon Stimulation with Inactivated Poultry Vaccines in Vitro. Vaccines 2020, Vol 8, Page 332 (2020) 8(2):332. doi: 10.3390/VACCINES8020332

27. van Dijk A, van Eldik M, Veldhuizen EJA, Tjeerdsma-van Bokhoven HLM, de Zoete MR, Bikker FJ, et al. Immunomodulatory and Anti-Inflammatory Activities of Chicken Cathelicidin-2 Derived Peptides. PLoS One (2016) II (2):e0I479I9. doi:I0.137I/journal.pone.0I47919

28. Setta A, Barrow PA, Kaiser P, Jones MA. Immune dynamics following infection of avian macrophages and epithelial cells with typhoidal and non-typhoidal Salmonella enterica serovars; bacterial invasion and persistence, nitric oxide and oxygen production, differential host gene expression, NF- $\kappa B$ signalling and cell cytotoxicity. Vet Immunol Immunopathol (20I2) I46(34):212-224. doi:I0.10I6/J.VETIMM.2012.03.008

29. Crippen TL. The selective inhibition of nitric oxide production in the avian macrophage cell line HDII. Vet Immunol Immunopathol (2006) I09(I-2):I27-I37. doi:I0.1016/j.vetimm.2005.08.024

30. He H, Genovese KJ, Swaggerty CL, Nisbet DJ, Kogut MH. A Comparative Study on Invasion, Survival, Modulation of Oxidative Burst, and Nitric Oxide Responses of Macrophages (HDII), and Systemic Infection in Chickens by Prevalent Poultry Salmonella Serovars. Foodborne Pathog Dis (20I2) 9(I2): I I04-I I I0. doi: 10.1089/fpd.20I2. I233

3I. He H, Genovese KJ, Kogut MH. Modulation of chicken macrophage effector function by $\mathrm{T}(\mathrm{H}) \mathrm{I} /$ $\mathrm{T}(\mathrm{H}) 2$ cytokines. Cytokine (20II) 53(3):363-369. doi:10.1016/j.cyto.2010.12.009

32. Beug H, von Kirchbach A, Döderlein G, Conscience J-F, Graf T. Chicken hematopoietic cells transformed by seven strains of defective avian leukemia viruses display three distinct phenotypes of differentiation. Cell (1979) 18(2):375-390. doi:10.1016/0092-8674(79)90057-6

33. Duff GW, Atkins $E$. The inhibitory effect of polymyxin B on endotoxin-induced endogenous pyrogen production. J Immunol Methods ( 1982) 52(3):333-340. doi: I 0. I0I6/0022-1759(82)900059

34. Ariaans MP, Matthijs MGR, van Haarlem D, van de Haar P, van Eck JHH, Hensen EJ, et al. The role of phagocytic cells in enhanced susceptibility of broilers to colibacillosis after Infectious Bronchitis Virus infection. Vet Immunol Immunopathol (2008) 123(3-4):240-250. doi: I0.1016/J. VETIMM.2008.02.003 
35. Livak KJ, Schmittgen TD. Analysis of Relative Gene Expression Data Using Real-Time Quantitative PCR and the 2- $\Delta \Delta C T$ Method. Methods (200I) 25(4):402-408. doi:I0.1006/meth.2001.1262

36. Eldaghayes I, Rothwell L, Williams A, Withers D, Balu S, Davison F, et al. Infectious Bursal Disease Virus: Strains That Differ in Virulence Differentially Modulate the Innate Immune Response to Infection in the Chicken Bursa. Viral Immunol (2006) I9(I):83-9I. doi:I0.1089/vim.2006.19.83

37. Degen WGJ, van Daal N, van Zuilekom HI, Burnside J, Schijns VEJC. Identification and Molecular Cloning of Functional Chicken IL- I2. J Immunol (2004) I72(7). doi: I0.4049/jimmunol. I72.7.437I

38. Truong AD, Hoang CT, Hong Y, Lee J, Lee K, Lillehoj HS, et al. Functional analyses of the interaction of chicken interleukin 23 subunit pl9 with IL- 12 subunit p40 to form the IL-23 complex. Mol Immunol (2017) 92. doi:10.1016/j.molimm.2017.09.019

39. Mittal SK, Roche PA. Suppression of antigen presentation by IL-10. Curr Opin Immunol (20I5) 34:22-27. doi:10.1016/j.coi.2014.12.009

40. Keestra AM, van Putten JPM. Unique Properties of the Chicken TLR4/MD-2 Complex: Selective Lipopolysaccharide Activation of the MyD88-Dependent Pathway. J Immunol (2008) I8I (6):43544362. doi: 10.4049/jimmunol.181.6.4354

4I. Boucher CE, Theron CW, Jansen AC, Bragg RR. Transcriptional profiling of chicken immunityrelated genes during infection with Avibacterium paragallinarum. Vet Immunol Immunopathol (2014) I58(3-4): I35-142. doi:10.1016/j.vetimm.2013.12.004

42. Droual R, Bickford AA, Charlton BR, Kuney DR. Investigation of problems associated with intramuscular breast injection of oil-adjuvanted killed vaccines in chickens. Avian Dis (1990) 34(2):473-478. doi:10.2307/159|439

43. Yamanaka M, Okabe T, Nakai M, Goto N. Local pathological reactions and immune response of chickens to ISA-70 and other adjuvants containing Newcastle disease virus antigen. Avian Dis (I993) 37(2):459-466. doi:10.2307/1591673

44. Reid GG, Blackall PJ. Comparison of adjuvants for an inactivated infectious coryza vaccine. Avian Dis $3 I(I): 59-63$.

45. Zhang L, Li P, Liu R, Zheng M, Sun Y, Wu D, et al. The Identification of Loci for Immune Traits in Chickens Using a Genome-Wide Association Study. PLoS One (2015) I0(3):e0I 17269. doi:10.1371/journal.pone.0117269

46. Canthaboo C, Xing D, Wei XQ, Corbel MJ. Investigation of role of nitric oxide in protection from Bordetella pertussis respiratory challenge. Infect Immun (2002) 70(2):679-684. doi:I0.I I28/ IAI.70.2.679-684.2002

47. Pinge-Filho P, Peron JPS, De Moura TR, Menolli RA, Graça VK, Estevão D, et al. Protective immunity against Trypanosoma cruzi provided by oral immunization with Phytomonas serpens: Role of nitric oxide. Immunol Lett (2005) 96(2):283-290. doi:I0.1016/j.imlet.2004.09.010

48. Petruzzi B, Dalloul RA, LeRoith T, Evans NP, Pierson FW, Inzana TJ. Biofilm formation and avian immune response following experimental acute and chronic avian cholera due to Pasteurella multocida. Vet Microbiol (2018) 222:1 I4-123. doi:10.1016/j.vetmic.2018.07.005

49. Doan T-D, Wang H-Y, Ke G-M, Cheng L-T. N-terminus of Flagellin Fused to an Antigen Improves Vaccine Efficacy against Pasteurella Multocida Infection in Chickens. Vaccines (2020) 8(2):283. doi: 10.3390/vaccines8020283

50. Zhou L, Ivanov II, Spolski R, Min R, Shenderov K, Egawa T, et al. IL-6 programs TH-I7 cell differentiation by promoting sequential engagement of the IL-2I and IL-23 pathways. Nat Immunol (2007) 8(9):967-974. doi: I0.1038/nil488

5I. Boucher CE, Theron CW, Hitzeroth AC, Bragg RR. Regulation of chicken immunity-related genes and host response profiles against Avibacterium paragallinarum pathogen challenge. Vet Immunol Immunopathol (20I5) I67(I-2):70-74. doi:I0.10I6/j.vetimm.20I5.06.005

52. Medzhitov R, Janeway CA. Innate immunity: impact on the adaptive immune response. Curr Opin Immunol (1997) 9(I):4-9. doi:10.1016/S0952-79I5(97)80I52-5

53. Iwasaki A, Medzhitov R. Toll-like receptor control of the adaptive immune responses. Nat Immunol (2004) 5(I0):987-995. doi:I0.1038/nil I I2 
Chapter 3 | The immunostimulatory capacity of $A v$. paragallinarum in multivalent vaccines

54. Manicassamy S, Pulendran B. Modulation of adaptive immunity with Toll-like receptors. Semin Immunol (2009) 2I(4): I85-193. doi:10.1016/j.smim.2009.05.005

55. Dalod M, Chelbi R, Malissen B, Lawrence T. Dendritic cell maturation: Functional specialization through signaling specificity and transcriptional programming. EMBO J (20 I4) 33(I0): I I04-I I I 6. doi: I0.1002/embj.20I488027

56. EDQM. European Pharmacopoeia. 10th ed. Strasbourgh, France: European Department for the Quality of Medicines; 2020. 


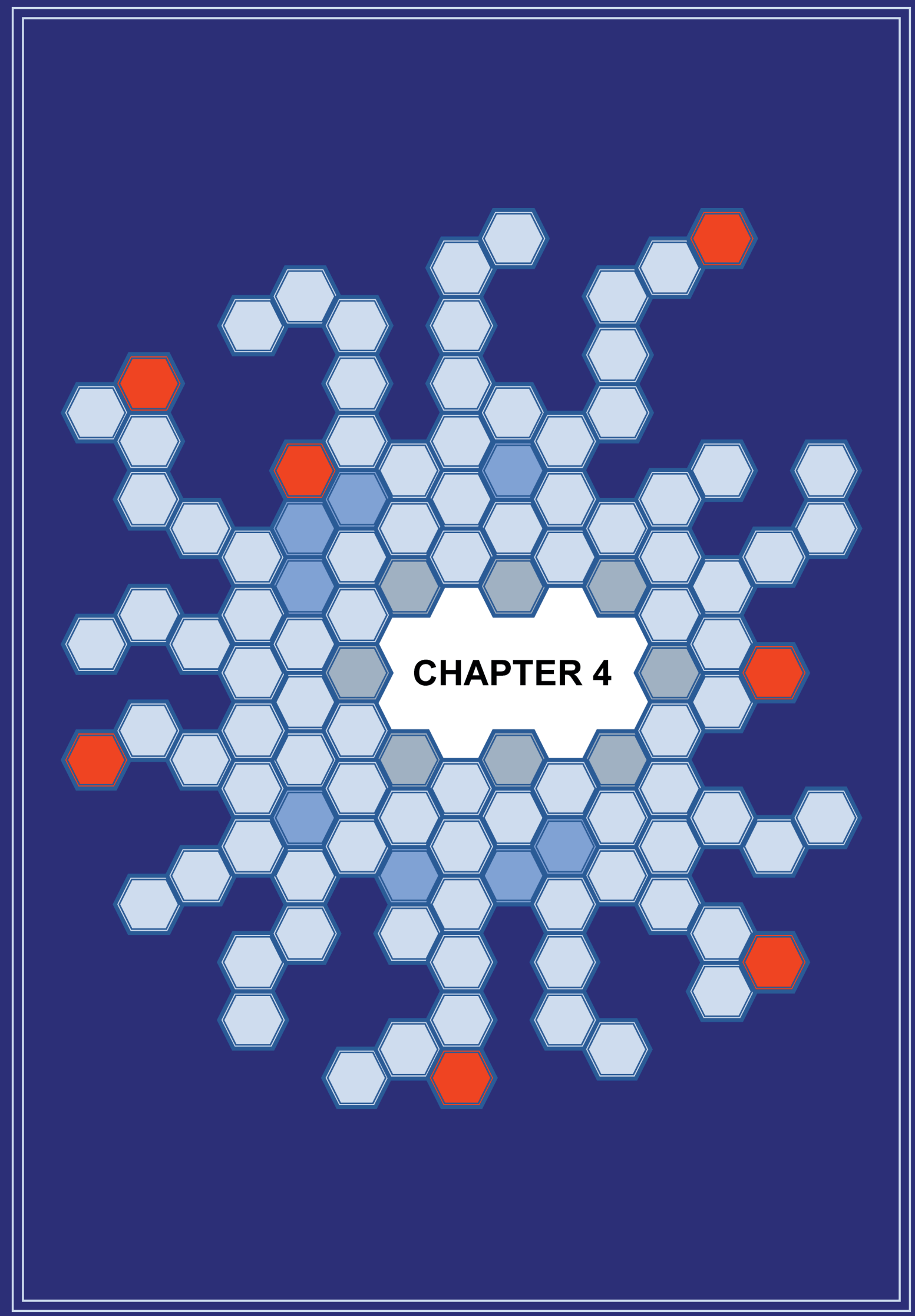


In vitro chicken bone marrow-derived dendritic cells comprise subsets at different states of maturation

Robin H.G.A. van den Biggelaar', Ger J.A. Arkesteijn', Victor P.M.G. Rutten $^{1,2}$, Willem van Eden' and Christine A. Jansen'

' Division of Infectious Diseases and Immunology, Department of Biomolecular Health Sciences, Faculty of Veterinary Medicine, Utrecht University, Utrecht, the Netherlands

${ }^{2}$ Department of Veterinary Tropical Diseases, Faculty of Veterinary Science, University of Pretoria, Pretoria, South Africa

Frontiers in Immunology 2020, I I, I 4 I https://doi.org//0.3389/fimmu.2020.00141 


\section{Abstract}

Research in chickens has been fundamental for the discovery of basic aspects of the immune system and has led to an interest in the in-depth characterization of avian immune cell types including dendritic cells (DCs). The in vitro generation and expansion of chicken bone marrow-derived DCs (chBMDCs) in the presence of granulocyte-macrophage colony-stimulating factor (GM-CSF) has provided a way to study chicken DCs, which are only present at limited cell numbers in vivo. This method has been employed to study the interactions between chicken DCs and pathogens or vaccines. However, a detailed characterization of the chBMDC culture is still lacking. In the present study, we performed an elaborate phenotypical and functional analysis of the chBMDC culture and addressed its heterogeneity. After 8 days of culture, chBMDCs comprised major histocompatibility complex class II (MHC-II) ${ }^{\text {low }}$ and $\mathrm{MHC}$-II ${ }^{\text {high }}$ subsets with different morphologies. Compared with MHC-II low chBMDCs, the MHC-II high subset showed a more mature phenotype, with higher expressions of CDI.I, CD40, CD80, CCR7, and CD83, and a relatively low opsonophagocytic capacity. Nevertheless, MHC-IIIigh chBMDCs did not show an increased capacity to induce T-cell proliferation. Therefore, MHC-II high chBMDCs were found to be semi-mature. Interestingly, the presence of the semi-mature MHC-II high chBMDC subset reduced when cells were cultured in the presence of IL-4. Finally, prolonged cell culture after fluorescence-activated cell sorting (FACS) converted the semi-mature MHC-II high subset back into the immature phenotype of the MHC-IIlow subset, demonstrating plasticity of their maturation state. This detailed characterization explained the heterogeneity of the chBMDC culture by the simultaneous presence of immature and semi-mature chBMDC subsets, in addition to cells without features of antigen-presenting cells. Our findings are instrumental for the interpretation of experiments using the chBMDC culture in past and future research by providing insights into its phenotypically and functionally distinct cell types. 


\section{Introduction}

Dendritic cells (DCs) are important innate immune cells that capture and process antigens to present them to cells of the adaptive immune system [I]. Adaptive immune responses result in pathogen-specific and long-lasting immunological memory, which enables the immune system to act more rapidly upon a second encounter with the pathogen. Vaccination against pathogens critically depends on DCs, which, respectively, support and fine-tune antigen presentation by co-stimulatory molecules and cytokines. The expression pattern of costimulatory molecules and cytokines by DCs depends on signals from their environment, including pathogen-associated molecular patterns (PAMPs) [I, 2] damage-associated molecular patterns (DAMPs) [3], and cytokines [I, 2, 4].

DCs are rare cells in all tissues and hard to isolate, which makes the use of primary DCs in functional assays challenging. To overcome this problem, granulocyte-macrophage colonystimulating factor (GM-CSF)-differentiated bone marrow-derived DC (BMDC) culture methods have been developed to generate DCs in large numbers [5]. The availability of cultured BMDCs facilitates their use in in vitro screening methods, including immunogenicity tests for vaccines and toxicity tests for allergens [6-8]. In addition to well-characterized murine BMDC culture methods, such methods have been developed for veterinary species, including dogs [9], cats [I0], cattle [II], sheep [I2], pigs [I3], and chickens [14].

Chicken BMDCs (chBMDCs) have been cultured in the presence of both recombinant chicken GM-CSF and interleukin-4 (IL-4) and were defined as DCs because of their typical stellate morphology and high expression of both major histocompatibility complex class II (MHC-II) and CDIIb/c [I4]. This chBMDC culture method has led to several studies into the role of chicken DCs in infection and vaccination. Maturation of chBMDCs has been observed after stimulation with lipopolysaccharide (LPS) or CD40L, as demonstrated by increased surface expression of co-stimulatory molecules CD40, CD83, and CD86; reduced phagocytosis and endocytosis; and an increased ability to induce a mixed lymphocyte reaction [14]. Similarly, chBMDCs have been found to mature upon exposure to avian influenza virus [15, 16], infectious bursal disease virus [17], or Salmonella enteritidis and Salmonella gallinarum vaccine candidates $[18,19]$.

Despite the widespread use of BMDCs originating from chickens and other species, a recent transcriptome study showed that murine GM-CSF-differentiated BMDCs differ phenotypically from murine DC populations in vivo [20]. Moreover, this study revealed that murine BMDC cultures comprise both CDI I b ${ }^{\text {high }}$ MHC-Illow macrophage-like and CDI I b low MHC-II ${ }^{\text {high }} \mathrm{DC}$-like subsets that are closely related, but still phenotypically and functionally different. These findings had implications for conclusions drawn using in vitro murine BMDC cultures as a model for DC biology in vivo and are part of the ongoing discussion on how to distinguish DCs and macrophages [20-25]. In addition, these findings stressed the importance of thorough characterization of the cellular subsets present in in vitro BMDC cultures and triggered us to explore in depth the nature of chBMDCs raised in vitro with GM-CSF and to determine whether these indeed represent DC-like cells.

The initial results of the present study showed that the chBMDC culture was heterogeneous and comprised MHC-IIlow and MHC-II high subsets, similar to observations in murine 
BMDC cultures. Therefore, we hypothesized that chBMDC culture comprised MHC-Illow macrophage-like and MHC-II ${ }^{\text {high }}$ DC-like subsets. However, in contrast to murine BMDC cultures, the MHC-IIlow and MHC-II ${ }^{\text {high }}$ subsets of the chBMDC culture were found to reflect different maturation states rather than distinct cell types. MHC-II high $c h B M D C s$ were found to exhibit increased expression of costimulatory molecules, also in the absence of stimuli. These findings on chBMDCs may have important consequences for conclusions drawn in past and future studies that make use of the chBMDC culture as a model for in vivo DC biology in chickens, in particular studies that assess chBMDC maturation.

\section{Materials and Methods}

\section{Bone marrow isolation}

Eighteen-day-old embryonated NOVOgen Brown eggs were obtained from a commercial breeder (Verbeek Broederij, Zeewolde, the Netherlands). Chicken embryos were removed from the eggs and euthanized by decapitation. Next, the tibiae and femurs were collected, bone heads were removed, and bone marrow was harvested by flushing the bones with RPMI-I640 cell culture medium supplemented with GlutaMAX ${ }^{\mathrm{TM}}-\mathrm{I}$, phenol red, and HEPES (Gibco ${ }^{\mathrm{TM}}$, Life Technologies Limited, Paisley, UK) under sterile conditions using a Plastipak $^{\mathrm{TM}}$ 10-ml syringe with a Microlance ${ }^{\mathrm{TM}} 3$ 2I-G needle (both from BD Biosciences, Pharmingen, San Diego, CA, USA). Bones and bone marrow cells were kept on ice during the whole procedure. Bone marrow cells from 200 embryos were pooled, gently squeezed through a Falcon ${ }^{\circledR} 70-\mu \mathrm{m}$ cell strainer (Corning®), Corning B.V. Life Sciences, Amsterdam, the Netherlands), and stored at $-140^{\circ} \mathrm{C}$ in RPMI, $50 \%$ chicken serum (Gibco ${ }^{\mathrm{TM}}$, Life Technologies Limited, Paisley, UK), and 10\% DMSO (Honeywell, Bucharest, Romania). This procedure resulted in batches comprising $1.3-2.3 \times 10^{9}$ bone marrow cells, which were frozen at a concentration of $2.5-5 \times 10^{7}$ cells per cryotube.

\section{chBMDC culture}

As previously described by others [26], chBMDCs were cultured from isolated bone marrow cells in RPMI- 1640 cell culture medium supplemented with $5 \%$ chicken serum and $50 \mathrm{U} / \mathrm{ml}$ of penicillin-streptomycin (all from Gibco ${ }^{\mathrm{TM}}$, Life Technologies Limited, Paisley, UK) in the presence of recombinant GM-CSF (and IL-4) at $41^{\circ} \mathrm{C}, 5 \% \mathrm{CO}_{2}$. Recombinant GM-CSF and IL-4 were produced using COS-7 cells transfected with pCl-neo (Promega Corporation, Madison, Wisconsin, USA) expressing the relevant cytokine, which were a kind gift from P. Kaiser and L. Rothwell (Roslin Institute, Edinburgh, UK). The concentrations of the recombinant cytokines are given as a dilution of supernatant from transfected COS7 cultures in accordance with a previous study [27]. GM-CSF was used at the titrated concentration $(2 \mu \mathrm{l} / \mathrm{ml})$ that resulted in the highest percentage of $\mathrm{MHC}-\mathrm{Il}^{+} \mathrm{CD} 40^{+} \mathrm{CD} 80^{+}$ cells. In one experiment, the chBMDC culture was supplemented with GM-CSF and titrated concentrations of IL-4. Bone marrow cells were seeded at $2.5 \times 10^{6}$ cells per milliliter in 75$\mathrm{cm} 2$ cell culture flasks in $15 \mathrm{ml}$ of RPMI- 1640 medium per flask, in $25-\mathrm{cm} 2$ cell culture flasks in $5 \mathrm{ml}$ of RPMI- 1640 medium per flask, in Costar $®$ six-well plates in $2 \mathrm{ml}$ of RPMI- 1640 medium per well, or in Costar® 24-well plates in $0.5 \mathrm{ml}$ of RPMI- 1640 medium per well (all from Corning®, Corning B.V. Life Sciences, Amsterdam, the Netherlands) depending on the required sample size. Early in the morning at day 3, culture medium with non-adherent cells was removed, and fresh RPMI- 1640 medium with GM-CSF (and IL-4) was added. Late in the afternoon at day 4, the cultures received another volume of RPMI- I 640 medium with 
GM-CSF (and IL-4). The morphology of chBMDCs was examined by light microscopy using an EVOS FL microscope (AMG, Mill Creek, Washington, USA). In selected experiments, chBMDC cultures were matured by $100 \mathrm{ng} / \mathrm{ml}$ of LPS O I27:B8 (Sigma-Aldrich, Saint Louis, MO, USA) stimulation for $24 \mathrm{~h}$ at day 7 . To harvest the cultures at day 8 , the medium with non-adherent cells was first collected. Subsequently, loosely adherent cells were washed and collected with Dulbecco's phosphate-buffered saline (DPBS) without calcium and magnesium (DPBS ${ }^{--}$; Lonza, Basel, Switzerland). Finally, the remaining adherent cells were incubated in DPBS $^{-/}$supplemented with $5 \mathrm{mM}$ UltraPure EDTA (Invitrogen ${ }^{\mathrm{TM}}$, Life Technologies Europe BV, Bleiswijk, the Netherlands) for 10 min at room temperature (RT) before being collected as well. All cell-containing fluids (cell culture medium, DPBS ${ }^{-1}$, and DPBS $^{-/} 5$ mM EDTA) obtained during the harvest procedure were pooled for subsequent experiments.

\section{Flow cytometry analysis and antibodies}

Antibodies and streptavidin conjugates used in this study are listed in Table I. All were diluted in fluorescence-activated cell sorting (FACS) buffer, containing DPBS ${ }^{-/-}+0.5 \%$ BSA and $0.005 \%$ NaN3 (both from Sigma-Aldrich, Saint Louis, MO, USA), which was used for staining and washing steps. Antibodies and streptavidin conjugates were used at titrated concentrations to stain $0.5-1.0 \times 10^{6}$ freshly harvested chBMDCs per $50 \mu \mathrm{l}$ for $20 \mathrm{~min}$ at $4^{\circ} \mathrm{C}$. Between staining steps, chBMDCs were washed twice with FACS buffer. To assess viability, the cells were first washed in $\mathrm{DPBS}^{-/-}$and then stained with Zombie Aqua Fixable Viability Dye (BioLegend Inc., San Diego, CA, USA) diluted in DPBS ${ }^{-1}$ for 20 min at $4^{\circ} \mathrm{C}$. From each sample, 50,000-100,000 chBMDCs were analyzed using a CytoFLEX LX flow cytometer, equipped with 375-, 405-, 488-, 56I-, 638-, and 808-nm lasers (Beckman Coulter Inc., Brea, CA, USA), Flowjo Software v. 10.5 (Flowjo LCC, Ashland, OR, USA), and Prism 7 (GraphPad Software Inc., San Diego, CA, USA).

\section{Immunofluorescence microscopy analysis}

Ethanol-cleaned 12-mm glass coverslips (Waldemar Knittel Glasbearbeitungs GmbH, Brunswick, Germany) were placed into the wells of a 24-well cell culture plate. Next, bone marrow cells were seeded and cultured in complete RPMI culture medium in the presence of recombinant GM-CSF at $41^{\circ} \mathrm{C}, 5 \% \mathrm{CO}_{2}$. At day 8, differentiated chBMDCs on glass coverslips were either washed three times with $\mathrm{DPBS}^{-1-}$ or first stained with fluorescently labeled lectin wheat germ agglutinin (WGA)-Alexa Fluor 488 (Invitrogen ${ }^{\mathrm{TM}}$, Life Technologies Europe BV, Bleiswijk, the Netherlands). Staining with WGA-Alexa Fluor 488 was performed by washing the cells twice with cold DPBS with calcium and magnesium, followed by staining with WGA-Alexa Fluor 488 diluted in DPBS with calcium and magnesium for 20 min at $4^{\circ} \mathrm{C}$. Fixation was performed in DPBS ${ }^{-1-}$ with $4 \%$ paraformaldehyde (Alfa Aesar, Haverhill, MA, USA) for $30 \mathrm{~min}$ at RT. Subsequently, the fixatives were quenched by washing the fixed samples three times with DPBS ${ }^{-/-}$and $10 \mathrm{mM}$ glycine (Merck Millipore, Burlington, MA, USA) and blocked in blocking buffer, containing DPBS ${ }^{-1-}, 0.05 \%$ Tween-20 (Sigma-Aldrich, Saint Louis, MO, USA), and $2 \%$ bovine serum albumin (Sigma-Aldrich, Saint Louis, MO, USA), overnight at $4^{\circ} \mathrm{C}$. The coverslips were stained with the cells faced-down on Parafilm in 25 $\mu \mathrm{l}$ of blocking buffer with primary antibodies for $2 \mathrm{~h}$ and secondary antibodies for $\mathrm{I} \mathrm{h}$ at RT (antibodies are listed in Table I). In addition, a nuclear staining was performed in $25 \mu \mathrm{l}$ of blocking buffer at $10 \mu \mathrm{g} / \mathrm{ml}$ with 4',6-diamidino-2-phenylindole (DAPI) (Sigma-Aldrich, Saint Louis, MO, USA) for 5 min at RT. Between staining steps, the samples were washed three 


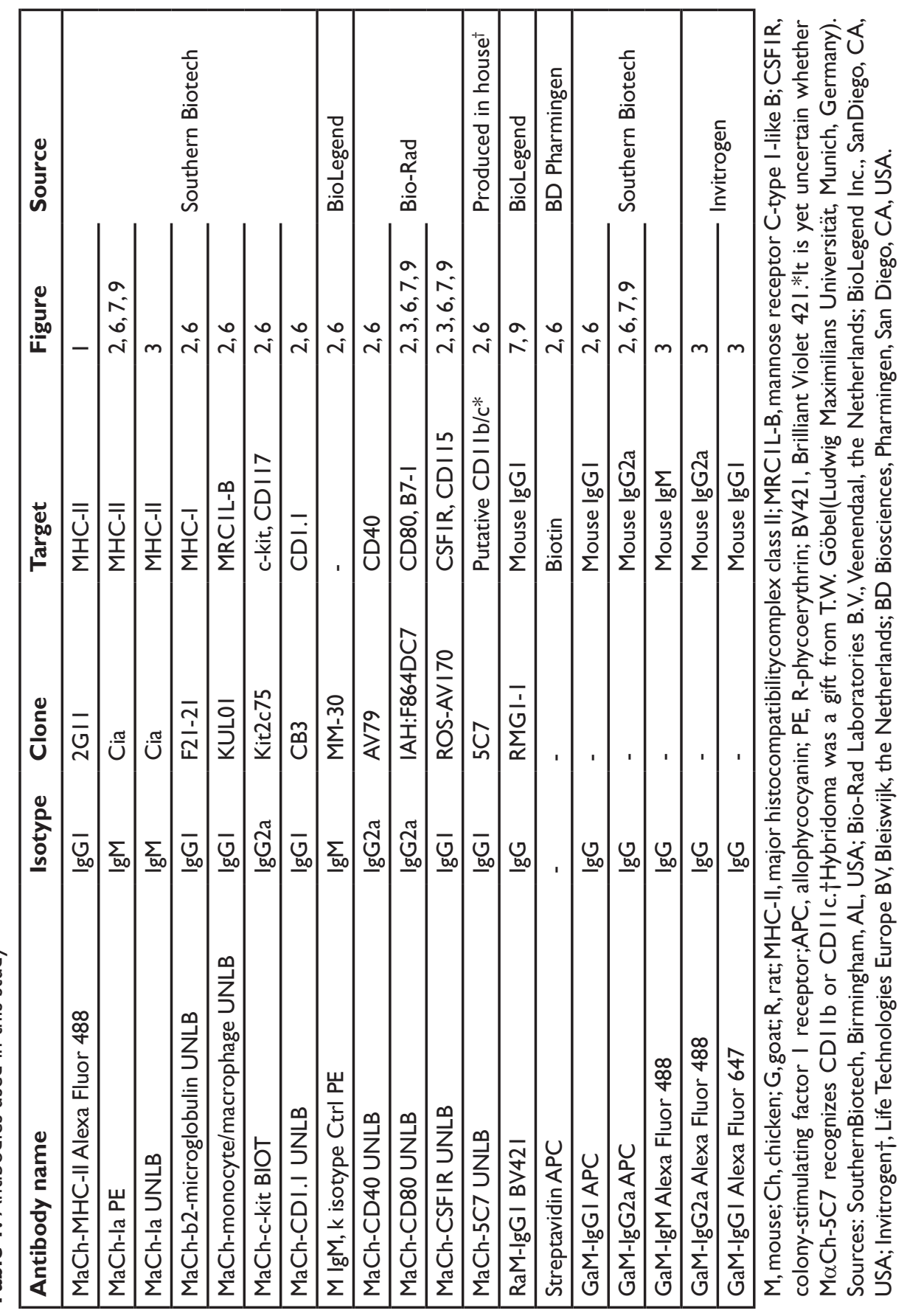


times with DPBS ${ }^{--}$with $0.05 \%$ Tween-20. The last wash step was performed in distilled water, before mounting the samples on Polysine $®$ microscope slides (Menzel Glaser $\mathrm{GmbH}$ \& Co KG, Braunschweig, Germany) in a FluorSave reagent (Calbiochem $®$, Merck Millipore, Burlington, MA, USA). The samples were captured using a TCS-SPE-II confocal microscope (Leica Microsystems B.V., Amsterdam, the Netherlands) equipped with 405-, 488-, 56I-, and 635-nm diode lasers and processed using Fiji software [28].

\section{Phagocytosis of IgY-opsonized beads by chBMDC subsets}

Chicken serum IgY fraction (Agrisera AB, Vännäs, Sweden) was added at $14.4 \mathrm{mg} / \mathrm{ml}$ to 1.44 $\times 10^{10}$ beads per milliliter of $\mathrm{I}-\mu \mathrm{m}$ crimson carboxylate-modified FluoSpheres (Invitrogen ${ }^{\mathrm{TM}}$, Life Technologies Europe BV, Bleiswijk, the Netherlands) and incubated overnight on an orbital shaker at $4^{\circ} \mathrm{C}$ to create lgY-opsonized beads. The next day, the beads were washed twice and resuspended in DPBS ${ }^{-1-}$ with centrifugation steps at $3,000 \times \mathrm{g}$ for 20 min at $4^{\circ} \mathrm{C}$ in between. To confirm IgY coupling, the beads were stained in FACS buffer with M $\alpha \mathrm{Ch}$ IgY-PE (SouthernBiotech, Birmingham, AL, USA) and analyzed on the CytoFLEX LX flow cytometer (data not shown). Next, the IgY-coupled beads were used in a phagocytosis assay to assess bead uptake by chBMDCs. After 8 days of culture in a 24-well plate, chBMDCs from one well were harvested and counted to determine the number of IgY-opsonized beads needed to obtain a I:I bead-to-cell ratio. Next, crimson beads were added to the remaining wells followed by 4 -h incubation at $41^{\circ} \mathrm{C}, 5 \% \mathrm{CO}_{2}$, to allow phagocytosis by chBMDCs. Subsequently, chBMDCs were harvested and stained for flow cytometry or confocal microscopy according to the methods described above. For flow cytometry, the cells were stained for MHC-II expression and viability, using Zombie Aqua Fixable Viability Dye, and analyzed using the CytoFLEX LX flow cytometer. For confocal microscopy, the cells were stained with WGA-Alexa Fluor 488, M $\alpha$ Ch-la BIOT, and streptavidin Alexa Fluor 405 and analyzed using the TCS-SPE-II confocal microscope.

\section{IL-4 bioactivity assessment by ${ }^{3} \mathrm{H}$-thymidine incorporation by PBMCs}

The ${ }^{3} \mathrm{H}$-thymidine incorporation assay to measure IL-4 bioactivity was modified from a published method to assess peripheral blood mononuclear cell (PBMC) proliferation [27]. Heparinized blood was collected from healthy chickens (under registration number AVDI08002016642-I from the Dutch Central Authority for Scientific Procedures on Animals). The chickens were daily monitored by animal caretakers for signs and symptoms of disease, which were absent for the chickens used in this study. PBMCs were isolated from heparinized blood by density gradient separation using Ficoll-Paque PLUS (GE Healthcare, Chicago, IL, USA) according to standard procedure. Collected PBMCs were resuspended in $2 \mathrm{ml}$ of Iscove's modified Dulbecco's medium (IMDM) culture medium supplemented with GlutaMAX ${ }^{\mathrm{TM}}$-I, phenol red, and HEPES, with $8 \%$ fetal bovine serum (FBS), $2 \%$ chicken serum, and $50 \mathrm{U} / \mathrm{ml}$ of penicillin-streptomycin (all from Gibco ${ }^{\mathrm{TM}}$, Life Technologies Limited, Paisley, UK). The cells were counted and seeded in a 96-well flat-bottom culture plate with $100 \mu \mathrm{l}$ of IMDM medium per well containing $2 \times 10^{5}$ cells. The cells received different concentrations (ranging I:25-I:250) of cell culture supernatant from COS-7 cells transfected with an IL-4 or empty $\mathrm{PCl}$ vector and were incubated for 4 days at $41^{\circ} \mathrm{C}, 5 \% \mathrm{CO}_{2}$. Subsequently, $0.4 \mu \mathrm{Ci}$ of ${ }^{3} \mathrm{H}$-thymidine per well was added to the culture for $18 \mathrm{~h}$, and the cells were harvested using a Harvester 96 (TOMTEC Imaging Systems GmbH, Unterschleißheim, Germany). ${ }^{3} \mathrm{H}$-Thymidine incorporation by the cells was determined in a I,450 MicroBeta Plus liquid scintillation counter (Wallac, PerkinElmer Life Sciences, Zaventem, Belgium). 


\section{Separation of chBMDC subsets by FACS}

For sorting, chBMDCs were stained with antibodies specific for MHC-II, colony-stimulating factor I receptor (CSFIR), and CD80 as before. In addition, the dye 7-aminoactinomycin $\mathrm{D}$ (7-AAD; BD Biosciences, Pharmingen, San Diego, CA, USA) was added to the cells for viability assessment. Next, the cells were resuspended in DPBS ${ }^{-1 /}$, I\% FBS, 2 mM EDTA, and $0.005 \% \mathrm{NaN} 3$ and flushed through a $70-\mu \mathrm{m}$ cell strainer to create single-cell suspensions. MHC-II /igh CSFIR ${ }^{\text {low }}$ and MHC-IIlow CSFIR Righ chBMDC subsets were sorted by FACS with a BD Influx cell sorter, equipped with 405-, 488-, 56I-, and 635-nm lasers (BD Biosciences, Pharmingen, San Diego, CA, USA). Each chBMDC subset constituted close to $25 \%$ of the original sample. Approximately $2 \times 10^{6}$ cells were sorted for both subsets to perform quantitative real-time PCR (RT-qPCR). In parallel, the cells were analyzed before and after FACS using the CytoFLEX LX flow cytometer. Moreover, some cells were sorted onto a Polysine $\AA$ microscope slide, fixed with $4 \%$ paraformaldehyde, and analyzed by confocal microscopy using the TCS-SPE-II microscope to confirm the expression patterns of MHC-II, CD80, and CSFIR by the chBMDC subsets. In addition, chBMDCs were sorted to evaluate the phenotypic stability of the cells by prolonged cell culture. Sorted chBMDCs were seeded into 24-well plates at 350,000 cells per well in I ml of RPMI culture medium with GM-CSF and incubation for I or 3 days of prolonged cell culture at $41^{\circ} \mathrm{C}, 5 \% \mathrm{CO}_{2}$, before repeated flow cytometric analysis.

\section{Gene expression analysis of separated chBMDC subsets using RT-qPCR} Sorted chBMDC subsets were lysed in RLT buffer (Qiagen GmbH, Hilden, Deutschland) and stored at $-20^{\circ} \mathrm{C}$ until RNA isolation. RNA isolation was performed with the RNeasy Mini Kit (Qiagen GmbH, Hilden, Deutschland) according to the manufacturer's instructions, including a DNase treatment step using the RNase-Free DNase Set (Qiagen GmbH, Hilden, Deutschland). Next, cDNA was prepared using the reverse transcriptase from the iScript cDNA Synthesis Kit (Bio-Rad Laboratories B.V., Veenendaal, the Netherlands) according to the manufacturer's instructions. RT-qPCRs were performed with primers (listed in Table 2) and either FAM-TAMRA-labeled TaqMan probes combined with TaqMan Universal PCR Master Mix or SYBR Green Master Mix without probes (all from Life Technologies Europe BV, Bleiswijk, the Netherlands). RT-qPCRs were performed with a CFX Connect and analyzed with the CFX Maestro software (both from Bio-Rad Laboratories B.V., Veenendaal, the Netherlands). All RT-qPCRs were evaluated for proper amplification efficiency (95$105 \%$ ) using serial dilutions of reference cDNA from splenocytes that were stimulated with concanavalin A for $24 \mathrm{~h}$ or from HDII cells that were stimulated with LPS for $3 \mathrm{~h}$. RTqPCRs were performed in triplo for every sample, and the average gene expression levels were expressed as $40-\mathrm{Ct}$ values, as described by Eldaghayes et al. [29]. The results were normalized toward gene expression levels of the housekeeping genes $28 \mathrm{~S}$ and GAPDH.

\section{Mixed lymphocyte reaction between chBMDC subsets and PBMCs}

PBMCs were isolated from heparinized blood by density gradient separation using FicollPaque PLUS according to standard procedure and resuspended in X-VIVO I5 cell culture medium. The cells were seeded in a 96 -well flat-bottom culture plate with 100,000 cells per well. Unsorted and sorted MHC-Illow and MHC-II high chBMDC subsets were resuspended in X-VIVO 15 medium and added to the PBMCs at different effector-to-target (E:T) ratios, ranging $1: 2-1: 8$. A positive control was created by addition of I $\mu \mathrm{g} / \mathrm{ml}$ of anti-CD3, I $\mu \mathrm{g} /$ $\mathrm{ml}$ of anti-CD28, and 1:50 supernatant from a COS-7 cells transfected with a $\mathrm{pCl}-$-neo 
Table 2. All primer and probe sequences are given from the 5' to 3' ends.

\begin{tabular}{|c|c|c|c|}
\hline Gene & NCBI Reference & Type & Sequences (5'-3') \\
\hline \multirow[t]{2}{*}{ MERTK } & NM_204988.I & Forward & TGTGGAAGGATGGCAGGGAG \\
\hline & & Reverse & GCACGGATGCTGAATGTAGAGG \\
\hline \multirow[t]{2}{*}{ ZBTB46 } & XM_0I52966I3.2 & Forward & CTGGACCTGTGGAAGAGGAAAC \\
\hline & & Reverse & CGGTAGTGGGAGGCAATCTC \\
\hline \multirow[t]{2}{*}{ iNOS } & NM_20496I.I & Forward & TGGGTGGAAGCCGAAATA \\
\hline & & Reverse & GTACCAGCCGTTGAAAGGAC \\
\hline \multirow[t]{2}{*}{ TLR4 } & NM_001030693.I & Forward & GTCCCTGCTGGCAGGAT \\
\hline & & Reverse & TGTCCTGTGCATCTGAAAGCT \\
\hline \multirow[t]{2}{*}{ GAPDH } & NM_204305.I & Forward & GTGGTGCTAAGCGTGTTATC \\
\hline & & Reverse & GCATGGACAGTGGTCATAAG \\
\hline \multirow[t]{3}{*}{ CDI4 } & NM_00II39478.I & Forward & GGACGACTCCACCATTGACAT \\
\hline & & Reverse & GGAGGACCTCAGGAACCAGAA \\
\hline & & Probe & AATGATCTTCCTGATTTGCAGACTGCCAA \\
\hline \multirow[t]{3}{*}{ CCR6 } & XM_0I5284I22.2 & Forward & GCCAGCCGCAGAAGAATGTA \\
\hline & & Reverse & TGTGGAGAAGAGTTTCAGAATGCT \\
\hline & & Probe & CAGAGTCGTGCAACATCGTCTGACCTACA \\
\hline \multirow[t]{3}{*}{ CCR7 } & NM_00I I98752.I & Forward & CATGGACGGCGGTAAACAG \\
\hline & & Reverse & TCATAGTCGTCGGTGACGTTGT \\
\hline & & Probe & TGAGGGTCACCATCGCTTTCAGCC \\
\hline \multirow[t]{3}{*}{ DEC205 } & NM_001037836.I & Forward & AACACGATGCCAGCTCTCAA \\
\hline & & Reverse & TTGACATGAAACGTAAGCTTCCTT \\
\hline & & Probe & CTACCAGTTCAACACCCAGTCTGCTCTTTCTTG \\
\hline \multirow[t]{3}{*}{ DC-SIGN } & NM_205484.I & Forward & TCTCGCTGAGCAGAATGAGTTG \\
\hline & & Reverse & GATGAGGTGGGAGTGCATCTC \\
\hline & & Probe & CACAAAGCGAAGGCGGAGTGCG \\
\hline \multirow[t]{3}{*}{ CD83 } & XM_418929.6 & Forward & TTGGCGACAGAATAGCATGG \\
\hline & & Reverse & CAGGGAGCCTCCAAGTCCTT \\
\hline & & Probe & AAGTCCTTGATGTGGAATCTCGTCATCCA \\
\hline \multirow[t]{3}{*}{$28 S$} & XR_003078040.I & Forward & GGCGAAGCCAGAGGAAACT \\
\hline & & Reverse & GACGACCGATTTGCACGTC \\
\hline & & Probe & AGGACCGCTACGGACCTCCACCA \\
\hline
\end{tabular}

In the absence of probe sequences, quantitative real-time PCR (RT-qPCR)was performed with SYBR Green Master Mix 
construct expressing chicken IL-2. A negative control was created by adding an additional X-VIVO 15 medium. The cells were cultured for 3 days at $41^{\circ} \mathrm{C}, 5 \% \mathrm{CO}_{2}$. Subsequently, $0.4 \mu \mathrm{Ci}$ of ${ }^{3} \mathrm{H}$-thymidine per well was added to the culture for $18 \mathrm{~h}$, and the cells were harvested using a Harvester. ${ }^{3} \mathrm{H}$-Thymidine incorporation by the cells was determined in a I,450 MicroBeta Plus liquid scintillation counter.

\section{Statistical analysis}

Statistical analysis was performed using GraphPad Prism 7 software (GraphPad Software, La Jolla, CA, USA). The data were tested for the assumptions of normally distributed data. Flow cytometry expression data showing the geometric mean fluorescent intensity (gMFI) was log-transformed in order to generate normally distributed data. Paired t-tests were used to test for statistically significant differences between MHC-II low and MHC-II high chBMDC subsets. A $p$-value of $<0.05$ was considered statistically significant.

\section{Results}

\section{chBMDC cultures are heterogeneous and comprise MHC-Illow and MHC- II $^{\text {high }}$ subsets cells that differ in morphology and phenotype}

Bone marrow cells, derived from bones of 18-day embryonated chicken embryos, were cultured in the presence of GM-CSF for 7 days to generate chBMDCs. The bone marrow isolates comprised mainly myeloid immune cells, including monocytes, thrombocytes, thromboblasts, granulocytes, and erythrocytes (Supplementary Figure SIa). After 7 days of culturing, nearly all cells were found to be $C D 45^{+}$and thus hematopoietic (Supplementary Figure SIb). In agreement with previous studies, chBMDCs formed clusters holding veiled cells (Figures Ia,c, arrows), typical for DC morphology [14], and highly expressing MHC-II (Figure Ib). MHC-II-expressing cell clusters did not appear in the absence of GM-CSF (Supplementary Figure S2). Beyond the clusters, cells with different morphologies were visible. These were large, round, without protrusions, and highly granular (Figure Ia, stars). Upon LPS stimulation, cell clusters became less dense, and many individual small cells with elongated protrusions became visible, a feature typical for mature DCs (Figure Id, arrows).

Next, a phenotypic analysis of the chBMDC culture by flow cytometry identified three subsets, distinguished by forward scatter (FSC) vs. MHC-Il expression: FSClow with no or

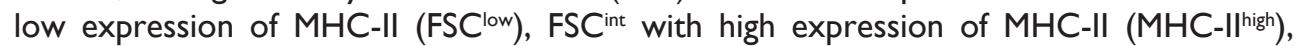
and FSC high with low expression of MHC-II (MHC-IIlow) (Figure 2a). These chBMDC subsets were evaluated for the expression of myeloid markers including integrin $\mathrm{CDI} / \mathrm{b} / \mathrm{c}$, costimulatory receptors CD40 and CD80, CSFIR, stem cell growth factor receptor c-kit, mannose receptor C-type I-like B (MRCIL-B), non-classical MHC molecule CDI.I, and MHC class I component $\beta 2$-microglobulin $(\beta 2 \mathrm{~m})$. FSClow cells showed high expression of CDIIb/c, but no expression of CD40 and CDI.I (Figure 2b). CD80, c-kit, MRCIL-B, and $\beta 2 \mathrm{~m}$ were expressed at moderate levels. CSFIR and c-kit were expressed by some FSClow cells, but not by others, showing further heterogeneity within this subset. Since FSClow cells were largely positive for MRCIL-B but showed no or low expression of MHC molecules and costimulatory molecules, these were likely to represent undifferentiated monocytes. Both MHC-II ${ }^{\text {high }}$ and MHC-II low cells showed expression of MHC molecules and 
(a)

(b)
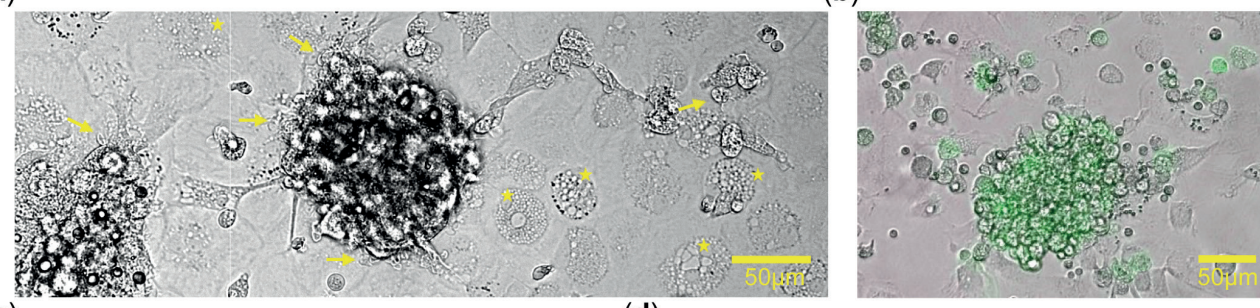

(c)

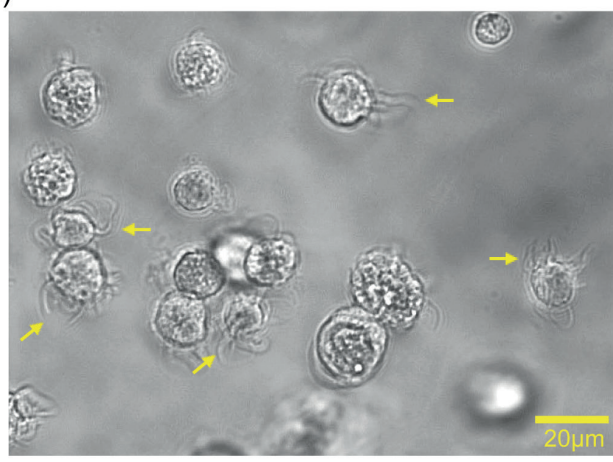

(d)

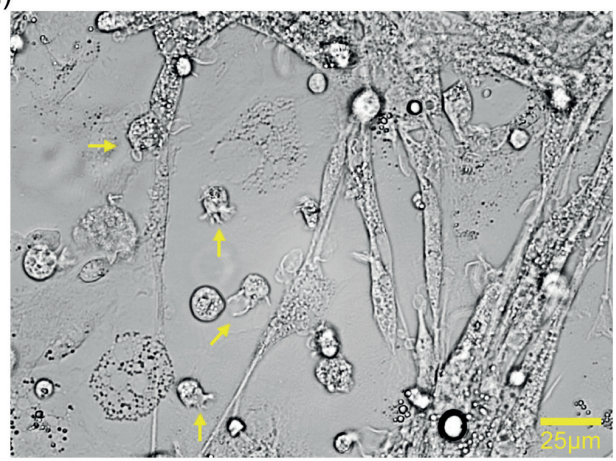

Figure I. Chicken bone marrow-derived dendritic cell (chBMDC) cultures contained clusters of major histocompatibility complex class II $\left(\mathrm{MHC}^{-I I}\right)^{+}$cells. (a) Bright-field light microscopy shows unstimulated chBMDCs after 7 days of culture. A cluster with chBMDCs is visible at the center. Yellow arrows indicate dendritic cells, visible as veiled cells that show protrusions. Yellow stars indicate highly granular cells without protrusions. (b) An overlay of light and fluorescent microscopy shows a cluster of unstimulated chBMDCs with MHC-II expression in green. (c) Light microscopy shows individual unstimulated chBMDCs that were transferred to a Petri dish after gentle resuspension in DPBS ${ }^{-1}$. (d) Light microscopy shows lipopolysaccharide (LPS)-stimulated chBMDCs.

costimulatory molecules. Compared to MHC-II low cells, MHC-II ${ }^{\text {high }}$ cells expressed higher levels of costimulatory receptors CD40 and CD80, CDI.I, and MRCIL-B (Figures 2b,c and Supplementary Table SI). Conversely, MHC-IIlow cells expressed higher levels of CDIIb/c, CSFIR, c-kit, and $\beta 2 \mathrm{~m}$. Taken together, MHC-II low and MHC-II high chBMDC subsets both showed a phenotype of antigen-presenting cells, but differentially expressed many myeloid markers.

Next, the expression patterns of CSFIR, MRCIL-B, and CD80 by MHC-IIlow and MHC-II high chBMDC subsets were evaluated by immunofluorescent confocal microscopy. The MHCII low subset expressed higher levels of CSFIR than the MHC-II high subset (Figure 3a), in accordance with the flow cytometry data (Figure 2 c). Therefore, CSFIR could be used as an additional marker to discriminate between chBMDC subsets. MHC-Illow CSFIR ${ }^{\text {high }}$ cells were found to be large and round and to have few protrusions, indicative of a macrophage-like morphology. Similar cells were observed by light microscopy (Figure I a, stars). In contrast, MHC-II high CSFIR low cells showed irregular shapes with many protrusions, indicative of a DC-like morphology, and resemble the veiled cells that were observed by light microscopy (Figure Ia). MRCIL-B and CD80 expression levels were found to be highest on MHCII high chBMDCs (Figures 3b,c), in accordance with the flow cytometry data (Figure 2c). Nevertheless, MRCIL-B and CD80 did not colocalize with MHC-II. MRCIL-B and CD80 were mainly found in intracellular compartments, whereas MHC-Il was found more on the 
(a)
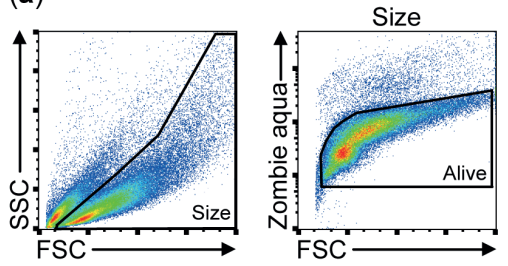

(b)
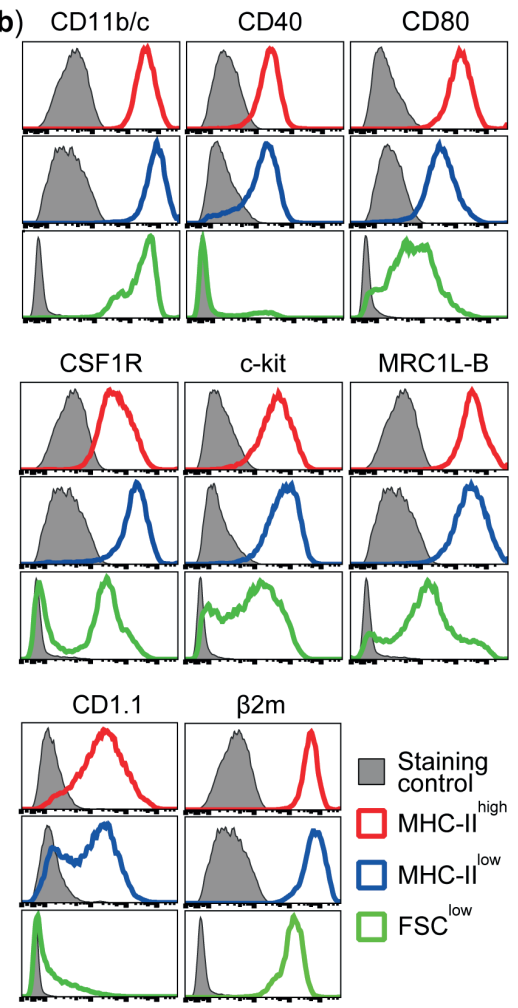

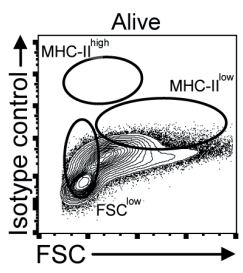

(c) $\mathrm{CD} 11 \mathrm{~b} / \mathrm{c}$

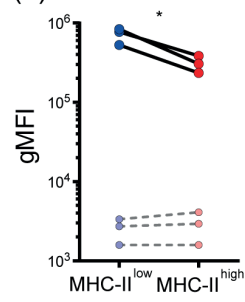

CSF1R

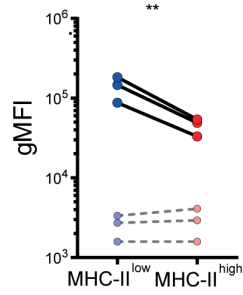

CD1.1

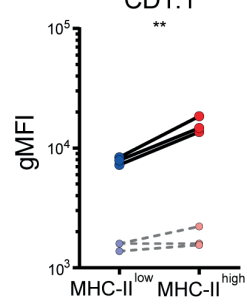

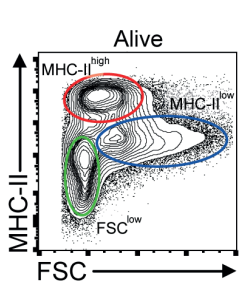
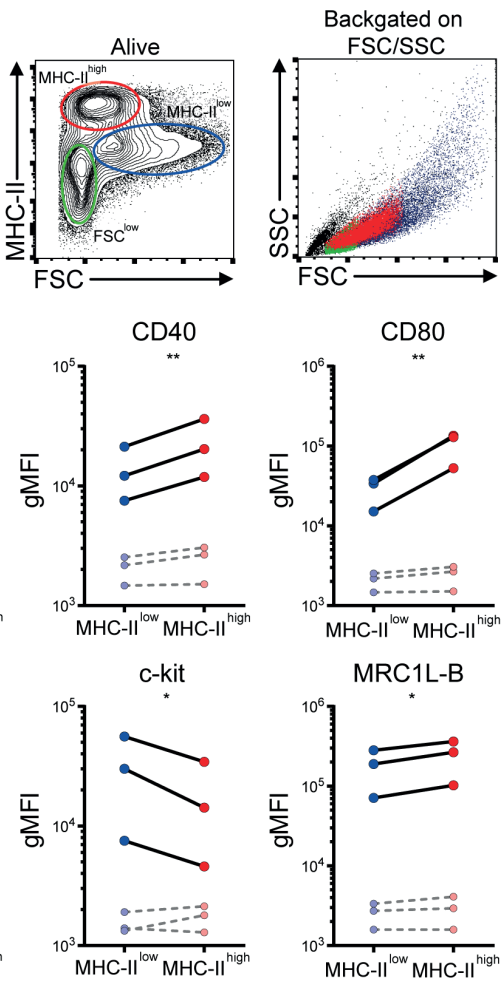

$\beta 2 \mathrm{~m}$

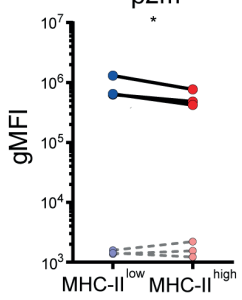

$\leftrightarrow$ Marker

Staining

Figure 2. Phenotypic analysis of chicken bone marrow-derived dendritic cells (chBMDCs) shows the presence of different subpopulations. (a) The cells were gated, respectively, for their scatter profile [forward scatter (FSC)/side scatter (SSC)], viability (Zombie Aqua), and expression of major histocompatibility complex class II (MHC-II), as shown in the first, second, and fourth panels. The third panel shows the signal after staining the cells with an isotype control antibody that corresponds to the antibody against MHC-II. Three subpopulations were selected based on FSC and MHC-II expression: $\mathrm{FSC}^{\text {low }}$ MHC-II-llow (green), FSCint MHC-II high (red), and FSChigh MHC-Illow (blue). The last panel shows the scatter profiles of the different subsets in matched colors, which were overlaid on the first panel. (b) Expression levels of a set of phenotypic markers are shown by representative histograms for the three subpopulations in corresponding colors. Each marker addressed was stained with a combination of either unconjugated primary antibody and allophycocyanin (APC)-conjugated secondary antibody or biotinylated primary antibody and APC-conjugated streptavidin. Filled gray histograms represent cells that have been stained with APC-conjugated streptavidin or secondary antibody to show the background fluorescence. (c) For the MHC-II' ${ }^{\text {low }}$ and MHC-I ligh subpopulations, the expression levels of all phenotypic markers are expressed as the geometric mean fluorescent intensity (gMFI) for three independent replicates. The gray dashed lines represent corresponding controls that have 
been stained with APC-conjugated streptavidin or secondary antibody alone. Statistically significant differences between chBMDC subsets are shown/indicated by ${ }^{*} \mathrm{p}<0.05$ and ${ }^{* *} \mathrm{p}<0.01$.

cellular surface of MHC-I/high-expressing chBMDCs. A stronger colocalization was observed between MRCIL-B and CD80 (Figure 3d).

\section{MHC-II low chBMDCs have a higher capacity to phagocytose fluorescent beads compared to MHC-II ${ }^{\text {high }}$ cells}

To assess whether the phenotypic distinction between MHC-IIlow and MHC-II high chBMDCs was functionally relevant, the subsets were assessed for their ability to phagocytose chicken IgY-coated fluorescent latex beads. First, the uptake of IgY-coated crimson fluorescent beads by chBMDCs was confirmed by showing that the beads localize beneath the surface of the plasma membrane, which was visualized using WGA (Figure 4a and Supplementary Video SI). chBMDCs were stained for MHC-II to identify the MHC-II ${ }^{\text {high }}$ subpopulation. Both MHC-IIlow and MHC-II high chBMDCs were found to take up beads as determined by confocal immunofluorescent microscopy. Next, the bead content of the chBMDC subsets was quantified by flow cytometry (Figure $4 \mathrm{~b}$ ). On average, MHC-II low cells ( 0.54 beads per cell) contained 2.4 times more beads than MHC-II high cells ( 0.23 beads per cell), which shows that the MHC-IIlow and MHC-II high chBMDC subsets differ in opsonophagocytic capacity (Figure 4c). A major part of IgY beads actually bound to the cells instead of being taken up, as shown in a separate experiment performed at $4^{\circ} \mathrm{C}$ (Supplementary Figure S3). However, when bound IgY beads from the experiment performed at $4^{\circ} \mathrm{C}$ were subtracted from the experiment performed at $41^{\circ} \mathrm{C}, \mathrm{MHC}$ - Il ${ }^{\text {low }}$ cells were still found to take up 2.7 times more beads than MHC-Illigh cells. The FSClow chBMDC subset showed little uptake of beads ( 0.048 beads per cell) (Figure $4 \mathbf{b}$ ).

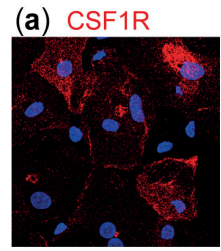

(c) $\mathrm{CD} 80$

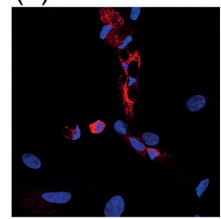

$\mathrm{MHC}-{ }^{\prime \prime}$

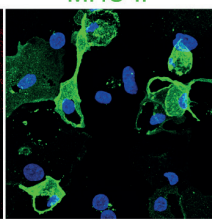

MHC-II

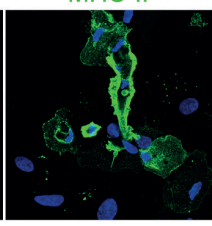

Merge

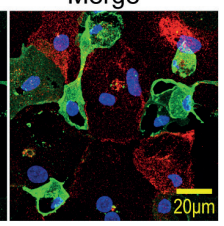

Merge

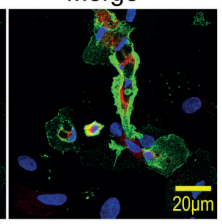

(b) MRC1L-B

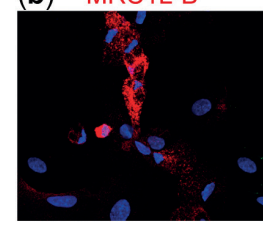

(d) MRC1L-B

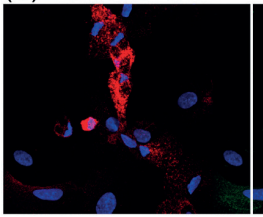

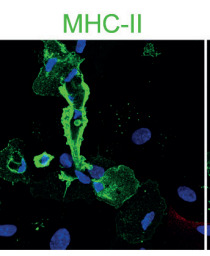

Merge

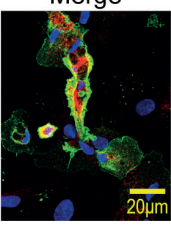

Merge

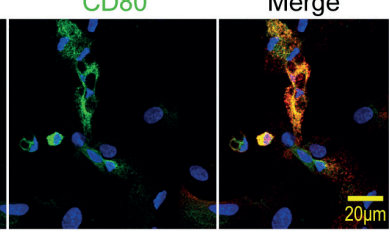

Figure 3. Mannose receptor C-type I-like B (MRCIL-B) and CD80 are expressed by irregularly shaped MHC-Il high chicken bone marrow-derived dendritic cells (chBMDCs), whereas colonystimulating factor I receptor (CSFIR) is expressed by large, round major histocompatibility complex class II (MHC-II) ${ }^{\text {low }}$ chBMDCs. (a) chBMDCs were stained for expression of CSFIR (red) and MHC-II (green). 4',6-Diamidino-2-phenylindole (DAPI) (blue) was used as a nuclear staining. A merged image shows potential colocalization between CSFIR and MHC-II. Similar images show the colocalization between MRCIL-B (red) and MHC-II (green) (b), between CD80 (red) and MHC-II (green) (c), and between MRCIL-B (red) and CD80 (green) (d). 
(a) MHC-II WGA Crimson beads Overlay

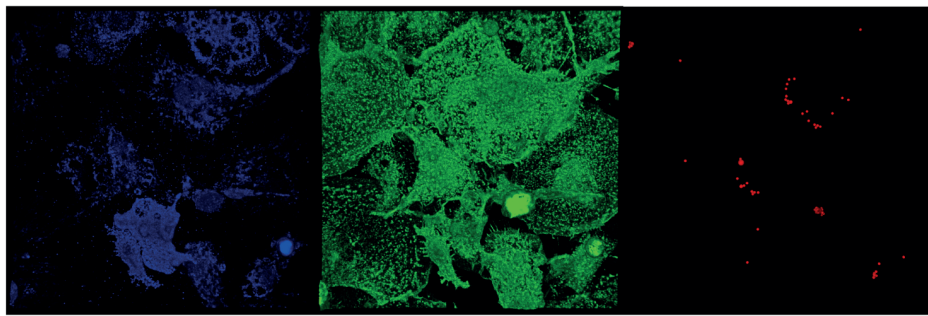

(b)
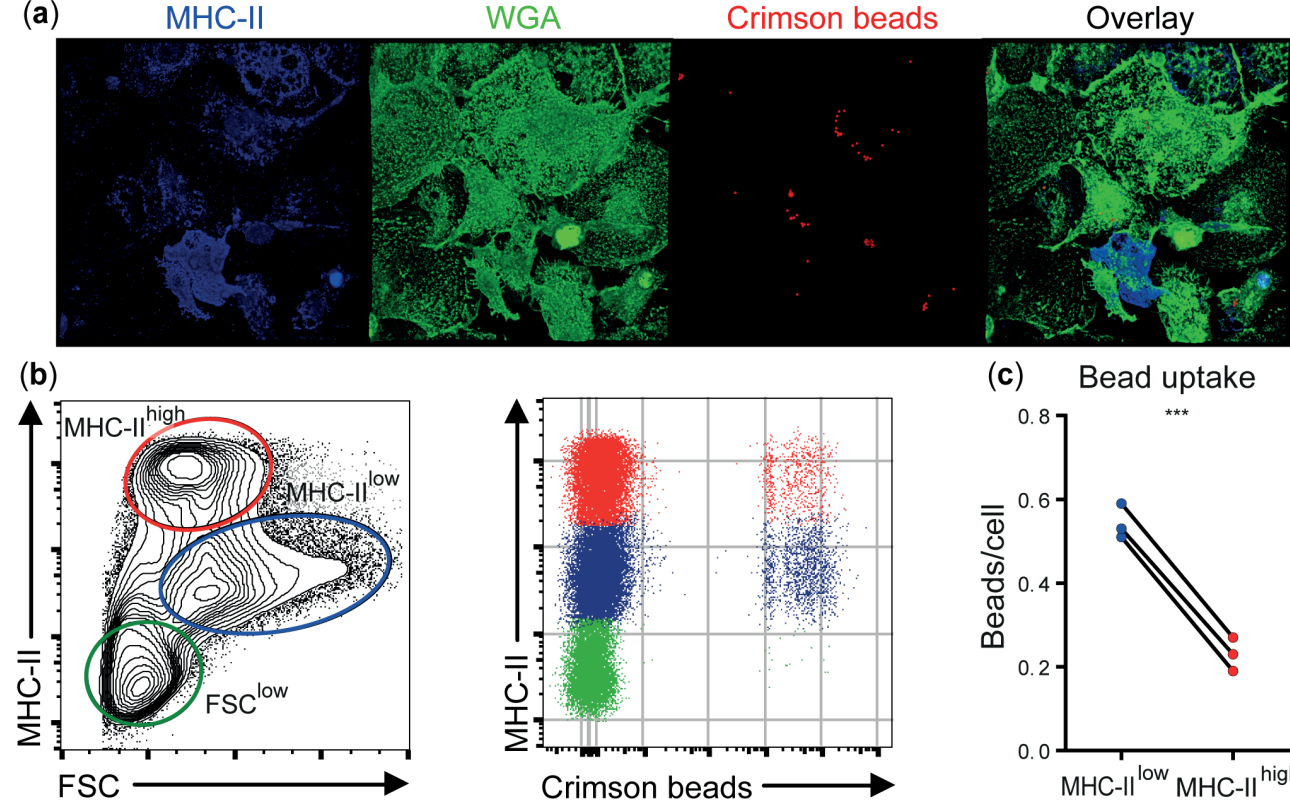

(c) Bead uptake

Figure 4. Major histocompatibility complex class II (MHC-II) ${ }^{\text {low }}$ chicken bone marrow-derived dendritic cells (chBMDCs) phagocytose IgY-coated beads more efficiently than MHC-I ligh chBMDCs. (a) chBMDCs were stained with wheat germ agglutinin (WGA) to visualize the plasma membrane, shown in green, and MHC-Il to identify MHC-II high cells, shown in blue, by confocal microscopy. Fluorescent crimson beads are shown in red. A 3D image was constructed from 56 z-stacks over a total distance of $9.4 \mu \mathrm{m}$. Here, a top view on the 3D image is shown, whereas all dimensions are visible in Supplementary Video SI. (b) Next, the bead content was quantified by flow cytometry for the previously identified chBMDC subpopulations. (c) The average number of beads per cell was calculated for MHC-IIlow and MHC-IIIigh subpopulations by dividing the mean fluorescent intensity (MFI) of the fluorescent beads by the MFI of cells that contained one bead (visible in B as the first positive line of cells). Each line represents an independent replicate $(n=3)$. A statistically significant difference between chBMDC subsets is shown/indicated by $* * * p<0.001$.

\section{Addition of recombinant IL-4 to chBMDC cultures leads to a smaller proportion of MHC-II high cells}

The effect of recombinant IL-4 on the generation of chBMDC subsets was investigated, since this cytokine has been used to generate chBMDCs by others [14]. To confirm that recombinant IL-4, produced in COS-7 cells, was biologically active, its ability to induce PBMC proliferation was demonstrated (Supplementary Figure S4). Next, IL-4 was given to chBMDCs alone or in combination with GM-CSF. IL-4 alone led to few MHC-II-expressing clusters of chBMDCs (Supplementary Figure S2). IL-4 in combination with GM-CSF led to many MHC-II-expressing clusters, similar to GM-CSF alone. Next, the proportion of MHC-IIlow and MHC-II high cells was quantified by flow cytometry. The addition of IL-4 to the standard chBMDC culture with GM-CSF was found to increase the proportion of the MHCIl ${ }^{\text {low }}$ from 32.6 to $49.7 \%$ at the highest administered dose (I/25 dilution) (Figure 5a). The increase in the proportion of MHC-IIlow cells occurred largely at the expense of the MHCII $^{\text {high }}$ subset, which changed proportionally from 35.0 to $23.0 \%$ at the highest administered dose of IL-4 (Figure 5b). The proportion of FSClow cells remained fairly stable (data not shown). 

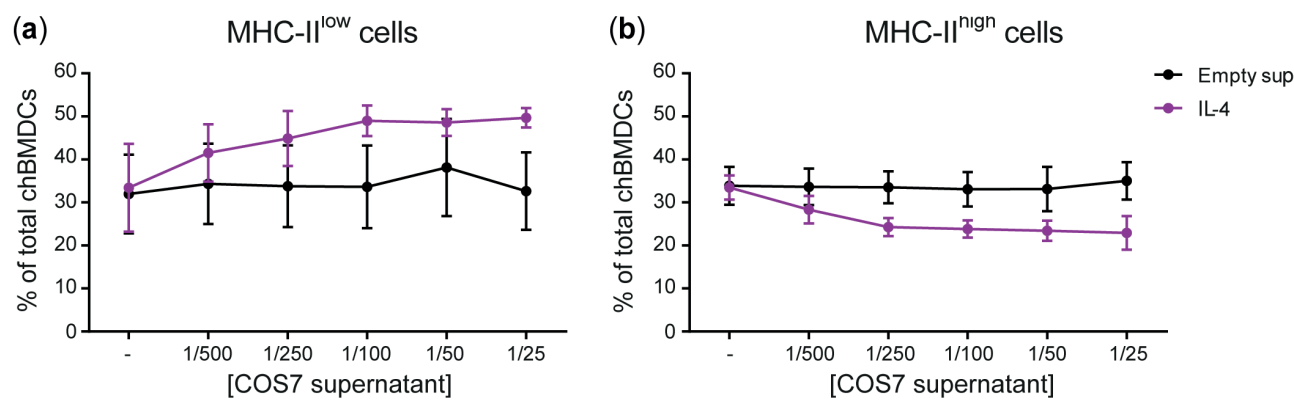

Figure 5. Addition of interleukin-4 (IL-4) to the culture medium during chicken bone marrow-derived dendritic cell (chBMDC) culture reduces the proportion of major histocompatibility complex class II (MHC-II) $)^{\text {high }}$ cells. The effect of IL-4 on MHC-IIlow (a) and MHC-II high chBMDC (b) subsets was assessed by flow cytometry. Supernatant from a COS-7 cell culture transfected with recombinant IL-4 (purple) was used as a source of IL-4, and supernatant from COS-7 cells transfected with an empty vector was used as a control (black). The graphs show the percentages of both chBMDC subsets within total chBMDCs after culturing the cells in the presence of titrated concentrations of IL-4 or empty supernatant control. Both panels show the mean of four independent replicates; the error bars of all panels show the SEM.

\section{LPS stimulation affects the difference in phenotype between MHC-II low and MHC-II high chBMDCs}

To determine the effect of LPS, commonly used to induce BMDC maturation, the cells cultured for 7 days were stimulated with $100 \mathrm{ng} / \mathrm{ml}$ of LPS for $24 \mathrm{~h}$. MHC-Illow and MHC-Il high chBMDC subsets were still detected (Figure 6a). Both MHC-Illow and MHC-Il high chBMDCs upregulated CD40, CDI.I, and $\beta 2 \mathrm{~m}$ expression, whereas the subsets downregulated c-kit and MRCIL-B expression (Figures $\mathbf{6 b}, \mathbf{c}$ ). CDI Ib/c, CSFIR, and c-kit expression on MHC-Illow cells decreased to levels similar to those on the MHC-Il high subset, while CD80 expression on MHC-I lligh cells decreased to a level similar to that on the MHC-Illow subset. These expression patterns suggest that the phenotypes of the MHC-Illow and MHC-II high subsets partially converged. Convergence in expression levels was also observed for CD40 and $\beta 2 \mathrm{~m}$, but not for MRCIL-B and CDI.I.

\section{chBMDC subsets differ in maturation status rather than cell type}

MHC-IIlow and MHC-Illigh chBMDC subsets were sorted by FACS to determine the differential expression of macrophage- and DC-related genes by RT-qPCR, since wellcharacterized monoclonal antibodies for these cell surface markers in chickens are scarce. Since MHC-II and CSFIR showed good discrimination between the subsets in confocal microscopy (Figure 3), these markers were used to separate the subsets by FACS (Figure 7a). In addition, chBMDCs were stained for CD80, which was found to be more highly expressed by the MHC-II high CSFIR low subset than the MHC-Illow CSFIR high subset (Figure 7a), in accordance with previous results (Figure 2c). Both subsets were sorted to above $90 \%$ purity as determined by flow cytometric reanalysis after each sort (Figure $\mathbf{7 b}$ ). Sorted cells were analyzed by fluorescent microscopy to confirm surface expression patterns of MHC-II, CSFIR, and CD80 (Figure 7c). In accordance with the flow cytometry data, MHCII and CD80 were expressed by the MHC-Il high CSFIR ${ }^{\text {low }}$ sorted subset, but not by the MHC-Il high CSFIR ${ }^{\text {low }}$ subset. In contrast, CSFIR was shown to be present on both sorted 
(a)

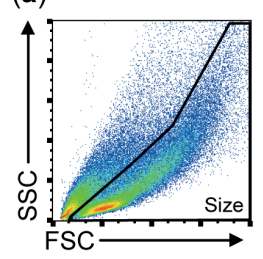

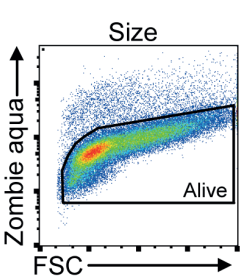

$\mathrm{CD} 40$

\section{(b)}

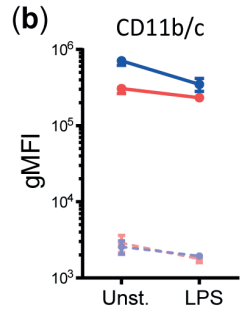

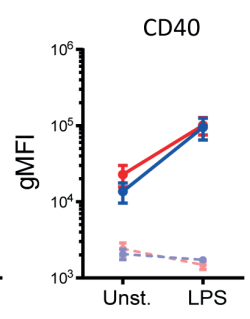
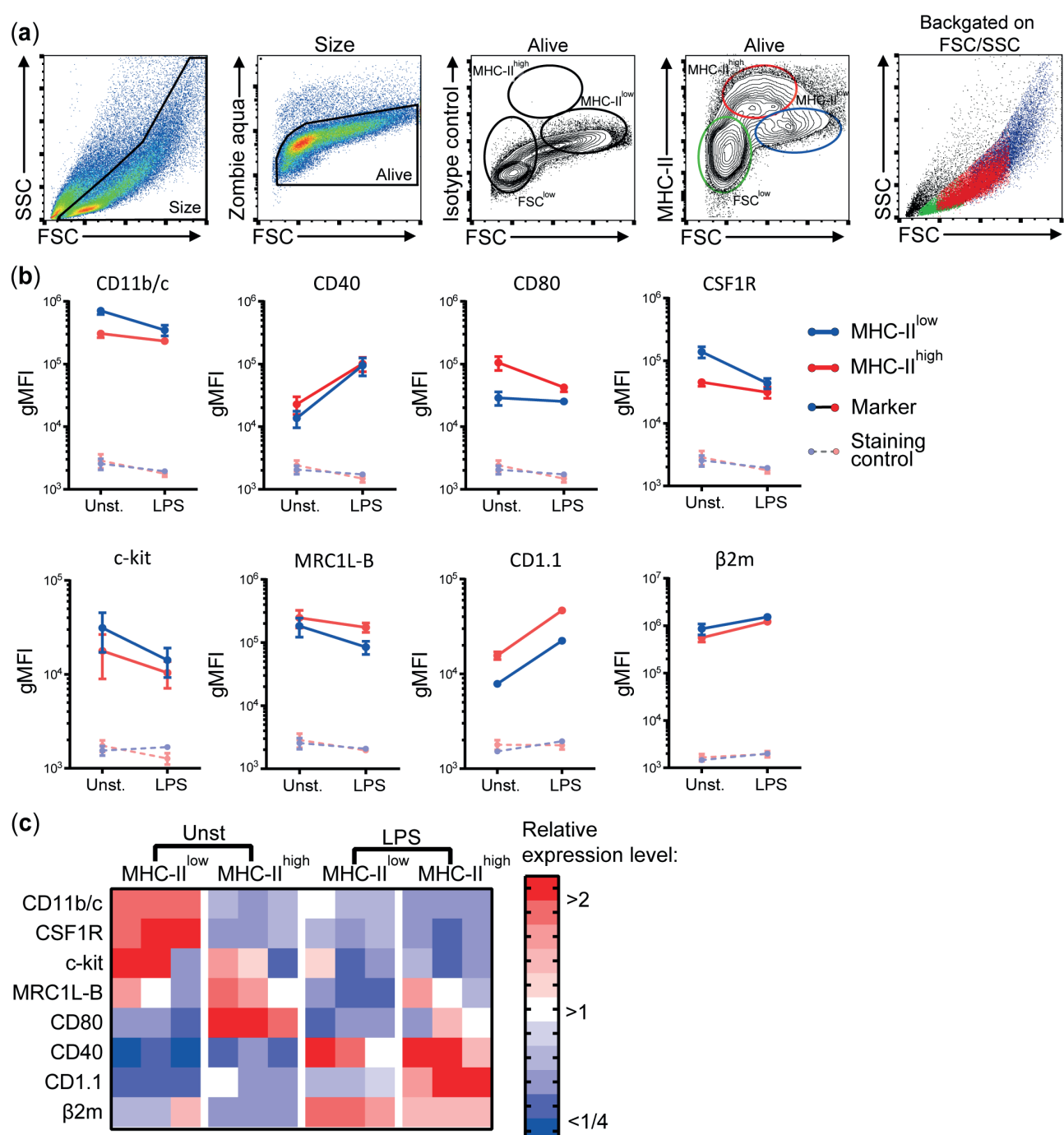

Relative expression level:

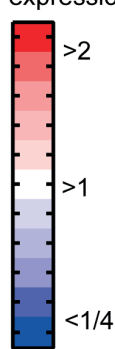

Figure 6. The phenotypic distinction between major histocompatibility complex class II (MHC-II) ${ }^{\text {low }}$ and MHC-Il high chicken bone marrow-derived dendritic cells (chBMDCs) is not retained after $24 \mathrm{~h}$ of lipopolysaccharide (LPS) stimulation. (a) LPS-stimulated chBMDCs were gated by a similar strategy as Figure 2A. (b) The change in myeloid marker expression upon LPS stimulation is shown for both MHC-II low and MHC-I lhigh chBMDC subsets. The error bars show the SEM of three independent replicates. (c) The expression of phenotypic markers is shown in a heat-map for unstimulated and LPS-stimulated MHC-IIlow and MHC-II high chBMDCs. To obtain the relative expression level for each marker, the gMFI of each sample was normalized to the average gMFI of all samples.

subsets. Next, RNA was isolated from the sorted subsets, and RT-qPCR was performed to study the gene expression patterns of macrophage- and DC-enriched genes (Figure 7d and Supplementary Table S2). Tyrosine-protein kinase Mer (MERTK), toll-like receptor 4 (TLR4), TLR4 coreceptor CDI4, and inducible nitric oxide synthase (iNOS) were used as macrophage-enriched genes, whereas zinc finger and BTB domain-containing protein 46 (ZBTB46), C-type lectins DEC205 and DC-SIGN, chemokine receptors C-C chemokine 
(a)

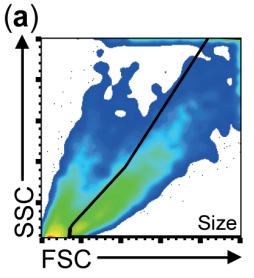

(b)
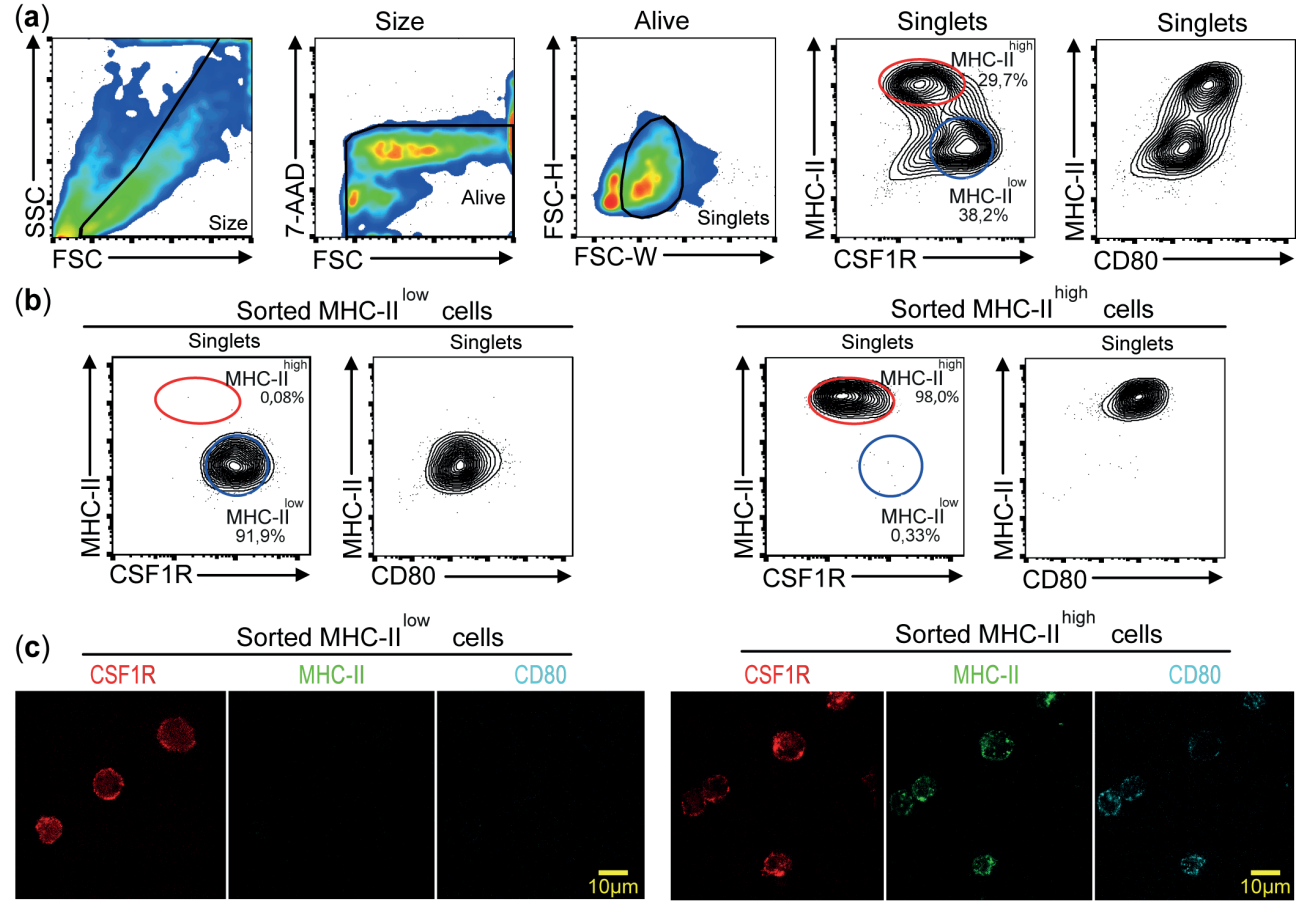

(d)

MERTK

TLR4

$C D 14$

iNOS

ZBTB46
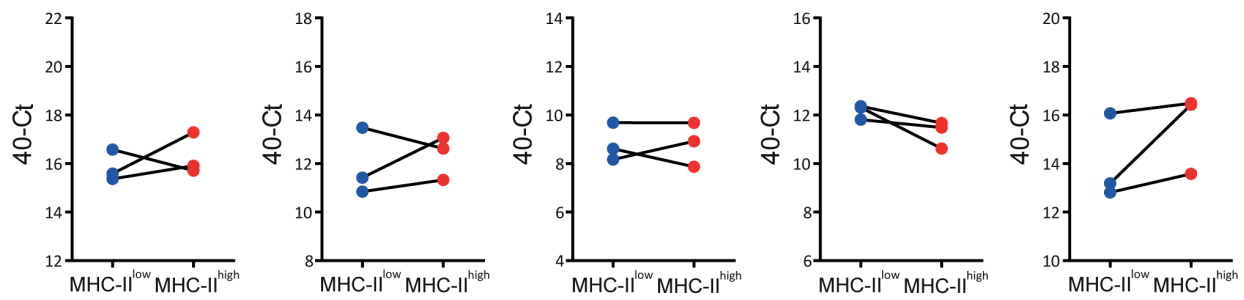

CCR6

CCR7
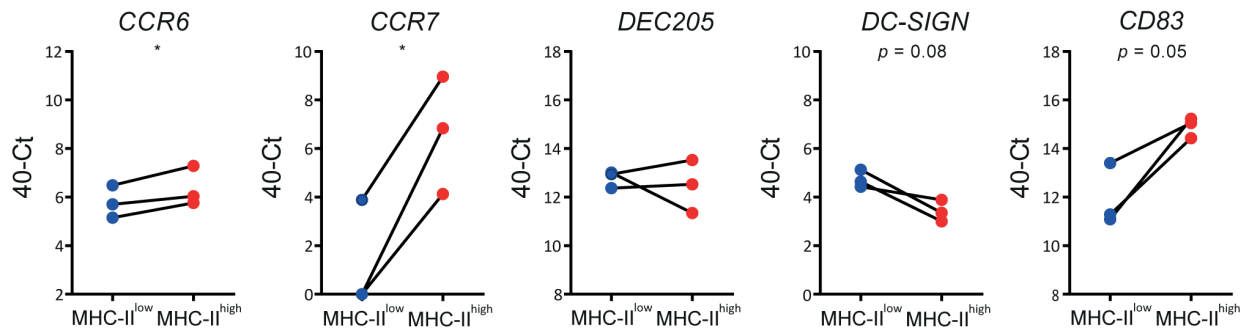

Figure 7. Quantitative real-time PCR (RT-qPCR) on sorted major histocompatibility complex class II (MHC-II) $)^{\text {low }}$ and MHC-II high chicken bone marrow-derived dendritic cell (chBMDC) subpopulations shows clear differential expression of DC maturation markers, but not of other DC- or macrophagespecific phenotypic markers. (a) Pre-sort evaluation of chBMDCs shows the subpopulations that differ in their expression of MHC-II, CSFIR, and CD80. The subpopulations were sorted after gating for scatter profile [forward scatter (FSC)/side scatter (SSC)], viability [7-aminoactinomycin D (7-AAD)], single cells (FSC-W/FSC-H), and expression of MHC-II and colony-stimulating factor I receptor (CSFIR). (b) Post-sort analysis of sorted MHC-IIlow and MHC-Il high chBMDC subpopulations shows that the sort led to populations of high purity. (c) Fluorescent microscopy shows CSFIR, MHC-II, and 
CD80 expressions for sorted MHC-IIlow and MHC-Iligh chBMDCs. Overlays and individual channels are shown with CSFIR in red, MHC-II in green, and CD80 in cyan. (d) RT-qPCR was performed on sorted MHC-IIlow and MHC-II high chBMDCs. The results are expressed at the 40-Ct value with $\mathrm{Ct}$ being the number of cycles needed to reach a signal above the threshold. The results were normalized for RNA content by the average expression of housekeeping genes $28 \mathrm{~S}$ and GAPDH. Each line represents an independent replicate $(n=3)$. Statistically significant differences between chBMDC subsets are shown/indicated by $*_{p}<0.05$. Non-significant $p$-values below 0.10 are given by the actual values to indicate trends close to significance.

receptor type 6 (CCR6) and 7 (CCR7), and costimulatory receptor CD83 were used as DC-enriched genes. Both subsets equally expressed MERTK $(d=0.46 ; p=0.60)$, TLR4 $(d$ $=0.42 ; p=0.62)$, CDI4 $(d=0 ; p>0.99)$, and DEC205 $(d=0.3 \mathrm{I} ; \mathrm{p}=0.70)$. MHC-Illow cells showed slightly higher expressions of iNOS $(d=0.90 ; p=0.16)$ and DC-SIGN $(d=1.32 ; p$ $=0.08)$, whereas MHC-II high cells showed slightly higher expressions of ZBTB46 $(\mathrm{d}=\mathrm{I} .48$; $p=0.24)$ and CCR6 $(d=0.58 ; p=0.05)$. More strikingly, MHC-II high cells showed much higher expressions of CCR7 $(d=5.35 ; p=0.02)$ and CD83 $(d=2.98 ; p=0.05)$. Thus, the differences between MHC-IIlow cells and MHC-II high cells were mainly found for DC-enriched genes, especially for CCR7 and CD83, which have been used by others before as maturation markers of chBMDCs [14, 30]. In contrast, differences in gene expression were hardly found for macrophage-enriched genes. Combined with the flow cytometric data (Figure 2c) that showed higher expressions of MHC-II, CD40, and CD80 by MHC-II high chBMDCs compared to the MHC-Ilow chBMDCs, the subsets seem to be DCs at different maturation states rather than different cell types.

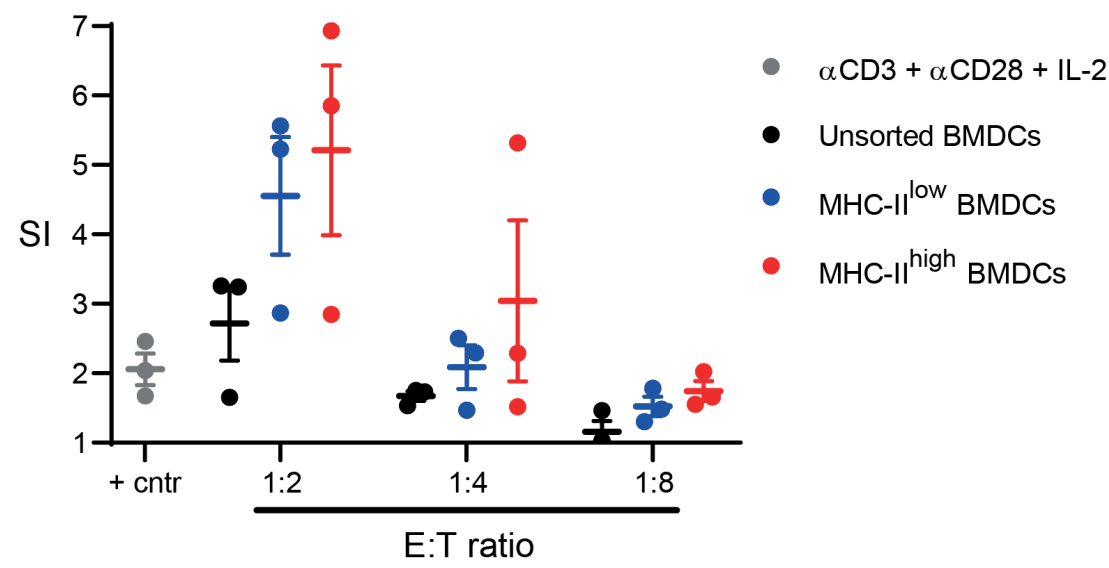

Figure 8. Sorted major histocompatibility complex class II (MHC-II) low and MHC-II high chBMDC subsets induce peripheral blood mononuclear cell (PBMC) proliferation in a mixed lymphocyte reaction. The MHC-Illow and MHC-Il' high chicken bone marrow-derived dendritic cell (chBMDC) subsets were sorted using the gating strategy shown in Figure 7a. Unsorted and sorted chBMDC effector cells were cocultured with PBMCs from laying hens $(n=3)$ at different E:T ratios. In addition, PBMCs were stimulated with a combination of anti-CD3, anti-CD28, and IL-2 as a positive control. After 3 days of culture, proliferation was measured by ${ }^{3} \mathrm{H}$-thymidine incorporation over a period of $18 \mathrm{~h}$ and expressed as a stimulation index (SI), which shows the ratio between stimulated and unstimulated PBMCs. All cultures were performed as three replicates. 
To determine whether chBMDCs were able to induce T-cell proliferation, allogeneic mixed lymphocyte reactions were performed with sorted chBMDC subsets. Both MHC-Ilow $\left(\mathrm{SI}=4.6\right.$ at I:2 E:T ratio) and $\mathrm{MHC}-\mathrm{II}^{\text {high }}(\mathrm{SI}=5.2$ at I:2 $\mathrm{E}: \mathrm{T}$ ratio) chBMDC subsets induced proliferation of PBMCs and did so more effectively than unsorted chBMDCs $(\mathrm{SI}=2.7)$ or PBMCs stimulated with a combination of anti-CD3, anti-CD28, and IL-2 (SI = 2.0) (Figure 8).

\section{MHC-II high chBMDCs become MHC-IIlow during prolonged incubation after sorting}

MHC-II low and MHC-II ${ }^{\text {high }}$ chBMDCs were found to differ in the expression of DC maturation markers, at both the protein and gene expression levels, but were similar in their ability to induce PBMCs to proliferate. To gain additional proof that MHC-Ilow and MHC-II high chBMDCs represent DCs at different maturation states rather than different cell types, sorted chBMDCs were reseeded for another I or 3 days of cell culture and stained again for MHC-II, CSFIR, and CD80. The MHC-II low subset showed only minor changes in the expression of abovementioned markers (Figures 9a,b). In contrast, the MHC-II ${ }^{\text {high }}$ subset showed higher CSFIR, lower MHC-II, and lower CD80 expression and consequently became phenotypically more similar to the MHC-Ilow subset. Therefore, both chBMDC subsets seem to consist of a DC-like cell type, but these appear to be different states of maturation with MHC-I/high chBMDCs being at a more mature but reversible state. In addition, the mRNA expression levels of CCR7 and CD83 were determined and found to be decreased for both subsets after prolonged cell culture (Figure 9c).

\section{Discussion}

The present study aimed to characterize GM-CSF-induced chBMDC cultures and address their heterogeneity. Despite the widespread use of in vitro grown DCs, there is still an ongoing debate about the representativeness of in vitro grown DCs for their in vivo counterparts [20-22, 24, 25, 3I]. In research so far, discussion focused mainly on BMDCs from mice and less on those of farm and companion animals. Early studies already described the presence of macrophage and granulocyte "contaminants" in the murine BMDC culture [5, 32]. Moreover, murine [20,33] and ovine [12] BMDC cultures, as well as bovine monocytederived DC cultures [34], have been shown to include CDI I b ${ }^{\text {low }}$ MHC-II ${ }^{\text {high }}$ cells with a DClike phenotype and CDI I bigh MHC-Illow cells with a macrophage-like phenotype. The first study by Wu et al. describing the chBMDC culture already recognized its heterogeneity [14]. However, this study excluded adherent and relatively small cells from analysis, which may, respectively, represent the MHC-IIlow chBMDCs and FSClow undifferentiated monocytes of the current study. In our opinion, these neglected cells should be characterized to interpret responses of the chBMDC culture correctly when analyzed in bulk. This has been illustrated by studies using LPS-stimulated murine BMDCs, in which individual cell subsets [20] or individual cells [35] were shown to respond very differently in their maturation, cytokine expression profile, and capacity to induce T-cell proliferation. Moreover, virus infection studies with chBMDCs, including avian influenza virus $[16,26]$ and infectious bursal disease virus [36] were analyzed in bulk, while the different chBMDC subsets might vary in their susceptibility for viruses and influence the outcome of these studies. 
(a)
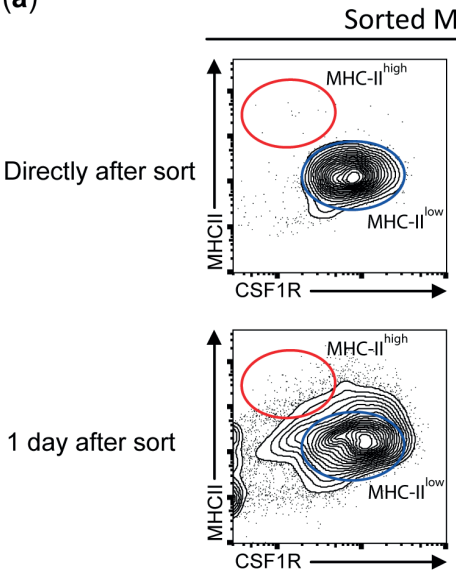

1 day after sort

3 days after sort

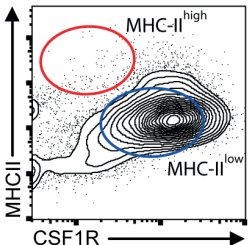

(b)

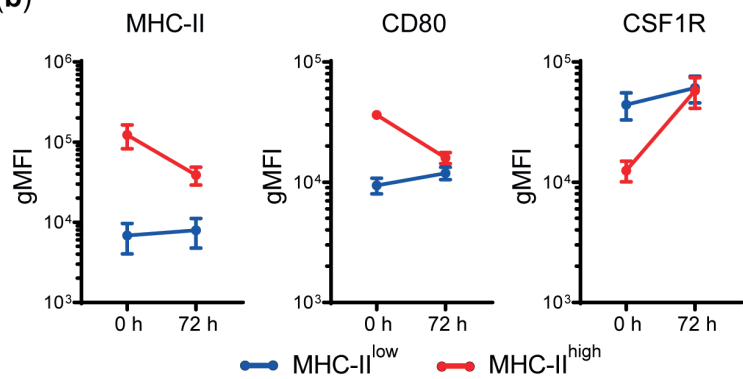

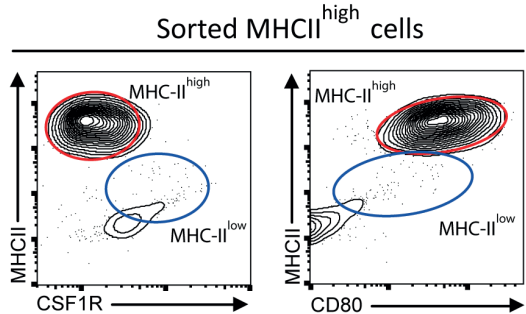
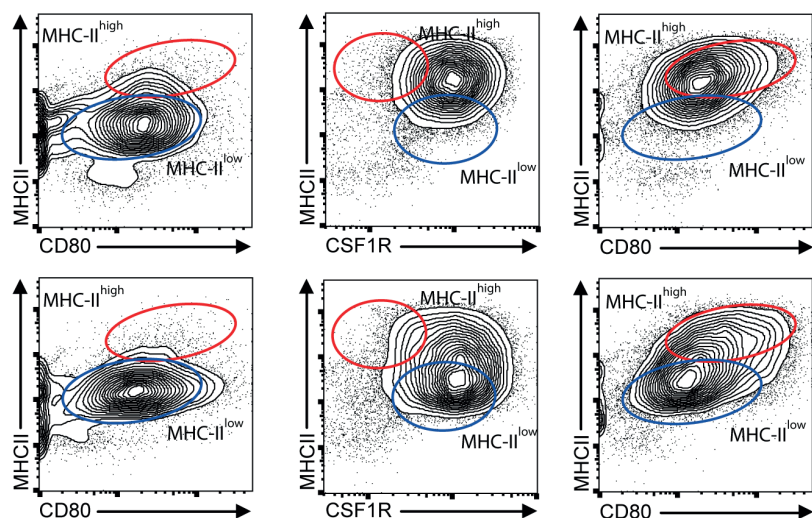

(c)

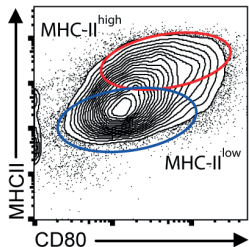

CD83
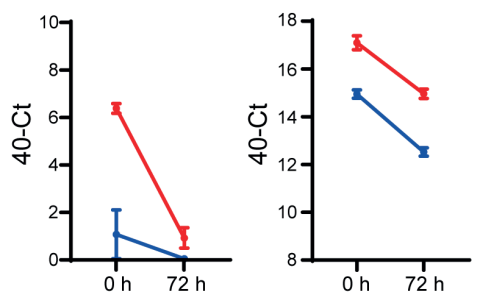

Figure 9. Sorted major histocompatibility complex class II (MHC-II) low chicken bone marrow-derived dendritic cells (chBMDCs) maintain their phenotype, while sorted MHC-Il'igh chBMDCs become MHC-IIlow. (a) The MHC-IIlow and MHC-II high chBMDC subsets were sorted using the gating strategy shown in Figure 7A. The cells were either reanalyzed directly after sorting or seeded for another I or 3 days of prolonged cell culture in the presence of granulocyte-macrophage colony-stimulating factor (GM-CSF). After these periods, the cells were stained again using the same protocol that was used before the sort. The contour plots that are shown are representative for three independent technical replicates. (b) Surface expression levels of MHC-II, CD80, and colony-stimulating factor I receptor (CSFIR) are shown for MHC-II low and MHC-II high chBMDCs directly after sorting $(0 \mathrm{~h})$ and after the 3 days of prolonged cell culture $(72 \mathrm{~h})(\mathrm{n}=3)$. (C) Similarly, messenger RNA (mRNA) expression levels of CD83 and CCR7 are shown for both subsets at 0 and $72 \mathrm{~h}$ (one experiment, performed in triplicate). 
In the present study, the chBMDC culture was found to contain MHC-IIlow and MHCII $^{\text {high }}$ subsets, similar to BMDC and monocyte-derived DC cultures in other species [I2, 20, 33, 34]. Expression of myeloid markers, morphology, and phagocytosis capacity differed between MHC-IIlow and MHC-II high subsets. Compared to the MHC-II ${ }^{\text {high }}$ subset, the MHC-IIlow subset showed higher expression of CSFIR, contained larger and more granular cells based on flow cytometry scatter profile and light microscopy, and showed a higher capacity to phagocytose IgY-opsonized beads. These results suggested that the MHC-Illow chBMDC subset consists of macrophage-like cells, in agreement with studies in murine [20,33] and ovine [12] BMDCs, as well as bovine monocyte-derived DCs [34]. In these studies, macrophage-like MHC-Illow cells showed high expression of CDIIb, which contributed to the distinction between the MHC-II' ${ }^{\text {low }}$ and $\mathrm{MHC}-\mathrm{II}^{\text {high }}$ subsets of these BMDC cultures. Unfortunately, this was not possible for the chicken CDIIb/c antigen, since the corresponding antibody has not yet been confirmed to recognize either CDI Ib or CDI IC [26]. Compared to the MHC-II low chBMDC subset, the MHC-II high subset showed higher expression of costimulatory molecules CD40 and CD80, in agreement with the DC-like phenotype shown for MHC-II ${ }^{\text {high }}$ cells in murine BMDC $[20,33]$ and bovine monocytederived DC cultures [34]. In addition, the MHC-II ${ }^{\text {high }}$ chBMDC subset showed a relatively high expression of the non-classical MHC molecule CDI.I, which is also indicative of a DC-like phenotype [37]. Our phenotypical findings of the chBMDC subsets suggested that

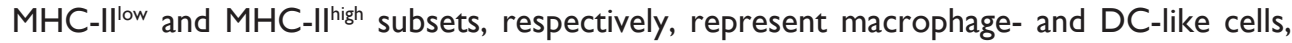
in agreement with studies that used murine, ovine, and bovine in vitro DC cultures [12, 20, 33, 34]. However, there is some discrepancy in the literature about murine BMDCs, since its $\mathrm{MHC}-$ II low $^{\text {lond }} \mathrm{MHC}$-II ${ }^{\text {high }}$ subsets have also been suggested to, respectively, represent an immature and mature phenotype of the same cell type $[3,38]$.

To further explore this alternative hypothesis, we investigated whether the chBMDC subsets were different cell types or DCs at different maturation states; $\mathrm{MHC}$ - $\mathrm{Il}^{\text {low }}$ and $\mathrm{MHC}$ II $^{\text {high }}$ subsets were sorted to perform RT-qPCR for macrophage- and DC-specific markers. Both subsets showed similar expression of the macrophage-specific markers MERTK, TLR4, and CDI4, which is in contrast to studies of murine BMDCs [20,33,39]. Moreover, no difference in expression of the DC-specific marker DEC205 was observed, while ZBTB46 and CCR6 were only moderately more highly expressed by the MHC-II high subset. The most striking differences between the chBMDC subsets were higher CD83 and CCR7 expressions and lower DC-SIGN expression for the MHC-II high subset. Increased CD83 and CCR7 expression and decreased DC-SIGN expression have been reported as maturation signatures of human and murine monocyte-derived DCs [20, 40, 4I], suggesting that the MHC-II high chBMDC subset represents a mature DC phenotype. Moreover, it has been shown that CCR7 expression is upregulated by chBMDCs shortly after LPS stimulation [36]. Taken together, these results suggest that, rather than being different cell types, MHC-IIlow and MHC-II high chBMDC subsets are DCs at different maturation states with MHC-II ${ }^{\text {high }}$ chBMDC being more mature. Nonetheless, MHC-IIlow and MHC-Illigh chBMDCs induced similar levels of PBMC proliferation in an allogeneic mixed lymphocyte reaction. Therefore, we hypothesize that of MHC-II high chBMDCs are in a semi-mature state, which is described in literature as the state at which DCs express high levels of MHC-II and costimulatory molecules but do not produce elevated levels of pro-inflammatory cytokines or optimally stimulate T-cell proliferation [42, 43]. Reseeding sorted chBMDCs showed that the immature phenotype of the $\mathrm{MHC}^{-I^{\text {low }}}$ subset remained stable over time, whereas 
the semi-mature MHC-IIhigh subset decreased MHC-II and CD80 expressions and increased CSFIR expression, indicating the plasticity and reversibility of this semi-mature phenotype. The chBMDC subsets differed in phagocytosis capacity, with the MHC-Illow subset being more efficient in bead uptake. This finding can also be explained by a different maturation status of the subsets, since mature DCs generally have a lower phagocytotic capacity [44, 45]. Others observed that LPS-induced maturation diminished the phagocytosis capacity of chBMDCs [14], which was not the case for the semi-mature MHC-II ${ }^{\text {high }}$ chBMDC subset of the present study.

The semi-mature phenotype of the MHC-II high chBMDCs must have been induced by the culture conditions that were used, since the cells were not intentionally stimulated. Since it is common practice to include IL-4 cytokine in BMDC differentiation protocols of different species, including chBMDCs [10-12, I4, 46, 47], the effect of IL-4 on the development of chBMDC subsets was investigated. When IL-4 alone was added to the culture, we observed that few chBMDC aggregates appeared, in agreement with previous studies [17]. Surprisingly, addition of IL-4 led to a lower proportion of MHC-II high cells, inhibiting chBMDC maturation, though the number of observed chBMDC aggregates remained unaffected. To the best of our knowledge, there are no earlier reports that show an inhibitory effect of IL-4 on maturation of the chBMDC culture in terms of MHC-Il expression. Previous studies have only observed the occurrence of chBMDC aggregates when investigating the effects of IL-4 $[14,17]$. Studies with murine and rat BMDCs have reported that IL-4 supplementation leads to proportionally larger MHC-II high subsets [20,47], in contrast to our findings for the chBMDC culture. Another study has shown that IL-4 has no effect on ovine BMDC yield or phenotype [12]. Therefore, the effect of IL-4 on BMDC cultures seems to differ between species. Another parameter affecting BMDCs was the source of the serum used in the culture. FBS was found to lead to a large MHC-II high $\mathrm{CD} 80^{+}$chBMDC population (Supplementary Figure S5), whereas chicken serum led to the immature MHC-II low and semi-mature MHC-II high chBMDCs of the present study.

BMDCs are often stimulated by LPS to induce maturation. In the chBMDC culture, LPS stimulation led to a striking increase in CD40 and CDI.I expression by both chBMDC subsets, whereas expression of maturation marker CD80 by the MHC-II high subset was unexpectedly decreased. Of note, LPS stimulation led to smaller differences between the subsets in their expression of MHC-II, CD80, and CSFIR. Overall, MHC-Illow and MHC-II high chBMDCs responded similarly to the LPS stimulus, which favored the hypothesis that the subsets reflected one cell type at different states. In contrast, murine $\mathrm{MHC}$ - $\mathrm{Il}^{\mathrm{low}}$ and $\mathrm{MHC}$ II $^{\text {high }}$ BMDC subsets were shown to maintain differential gene expression profiles after LPS stimulation, which provided additional proof that the murine subsets were truly different cell types [20].

In conclusion, this study describes the heterogeneity of the GM-CSF-differentiated chBMDC culture, which comprised MHC-II low and MHC-II high subsets that both possess features of antigen-presenting cells. These populations were found to differ in phenotype, morphology, and their phagocytosis capacity, whereas their ability to induce PBMC proliferation was similar. Based on higher expressions of maturation markers MHC-II, CD40, CD80, CD83, and CCR7 by MHC-Illigh chBMDCs compared to MHC-Illow chBMDCs, the MHCII low $^{\text {and } M H C-I I^{\text {high }}}$ subsets were found to, respectively, represent immature and semi- 
mature chBMDCs. The semi-mature phenotype of the MHC-Illigh subset was found to be reversible, since reseeding and prolonged culture of these cells led to a transition toward the immature phenotype of the MHC-Ilow cells. Taken together, these results yield a thorough characterization of the chBMDC culture and explain its heterogeneity by the simultaneous presence of immature and mature subsets. Our findings are instrumental for the interpretation of experiments that use this culture in future research.

\section{Acknowledgments}

The authors thank Alice E.J.A.M. Sijts for her critical reading of the manuscript and Irene $\mathrm{S}$. Ludwig for help with the ${ }^{3} \mathrm{H}$-thymidine incorporation assay. All fluorescent microscopy images have been acquired at the Center of Cellular Imaging, Faculty of Veterinary Medicine, Utrecht University.

\section{Funding}

This project has received funding from the Innovative Medicines Initiative 2 Joint Undertaking under grant agreement no. II5924 (VAC2VAC). This joint undertaking receives support from the European Union's Horizon 2020 research and innovation program and EFPIA. The contents of this article represent the authors' scientific conclusions, and neither IMI nor the European Union, EFPIA, or any associated partners are responsible for any use that may be made of the information contained therein. 


\section{Supplementary Materials}

(a)

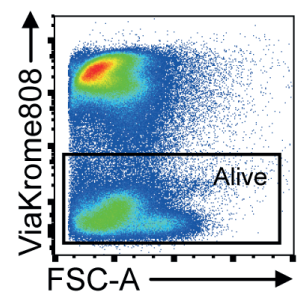

(b)

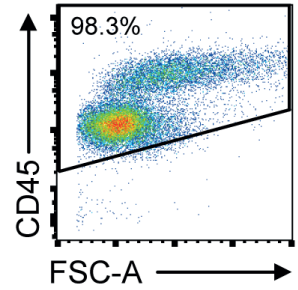

Alive
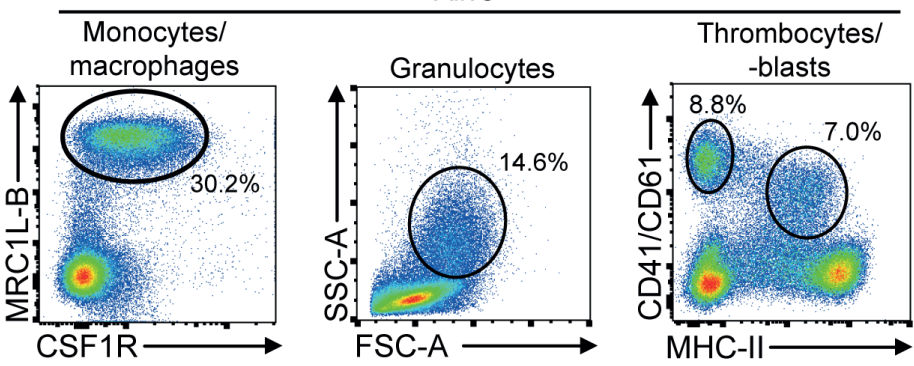
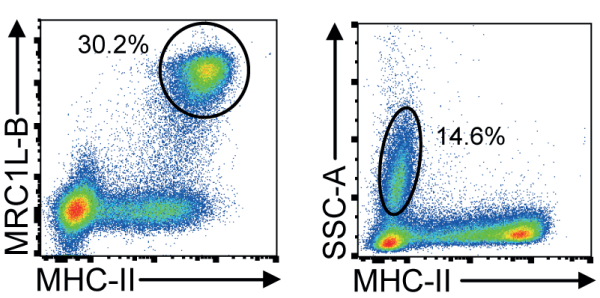

FSCA

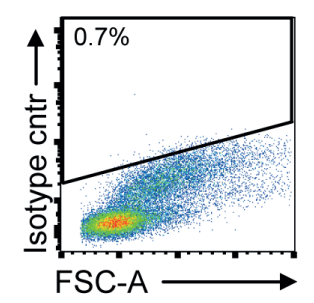

Supplementary Figure SI. Characterization of cells in an embryonic bone marrow isolate. (a) Embryonic bone marrow was characterized by flowcytometry to determine the presence of different subsets of myeloid cells. Cells from the monocyte/macrophage lineage were defined as MRCIL-B ${ }^{+}$ CSFIR+ MHC-II+, granulocytes as SSC high, thrombocytes as CD4I/CD6I+ MHC-II-, and thromboblasts as $\mathrm{CD} 4 \mathrm{I} / \mathrm{CD} 6 \mathrm{I}^{+} \mathrm{MHC}-\mathrm{Il}^{+}$. (b) After chBMDCs were cultured for 7 days in the presence of GM-CSF the proportion of hematopoietic cells was determined by CD45 staining. 

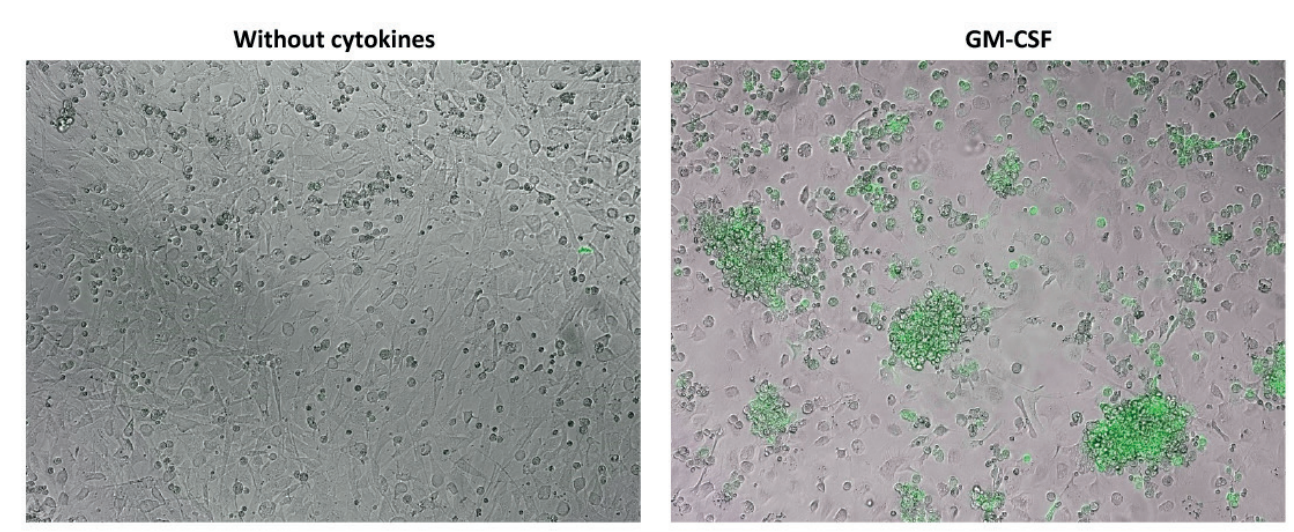

\section{IL-4}

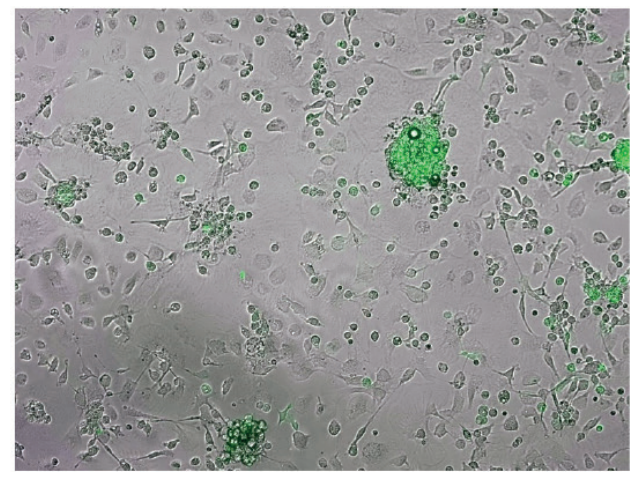

$\mathrm{IL}-4+$ + GM-CSF

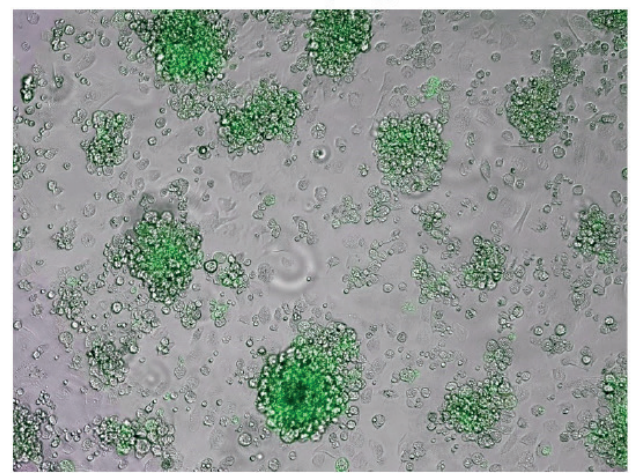

Supplementary Figure S2. The effects of GM-CSF and IL-4 on the morphology and MHC-II expression of the chBMDC culture. The chBMDC culture was performed in the absence of cytokines and in the presence of GM-CSF alone, IL-4 alone, or the combination of GM-CSF and IL-4. COS-7 cell culture supernatant containing the cytokines was added at $2 \mu \mathrm{l} / \mathrm{ml}$ for GM-CSF and at $5 \mu \mathrm{l} / \mathrm{ml}$ for IL-4. The chBMDC culture conditions were one or both cytokines were absent received an equivalent volume of supernatant from a COS-7 cell culture transfected with an empty $\mathrm{pCl}$-neo vector. At the end of the culture (day 8), an AlexaFluor488-conjugated mouse anti-chicken MHC-II monoclonal antibody was added to the cultures at $2 \mu \mathrm{g} / \mathrm{ml}$ and incubated for $30 \mathrm{~min}$ at $4^{\circ} \mathrm{C}$. Next, the cells were washed twice with DPBS with calcium and magnesium and analyzed using an EVOS FL microscope. 


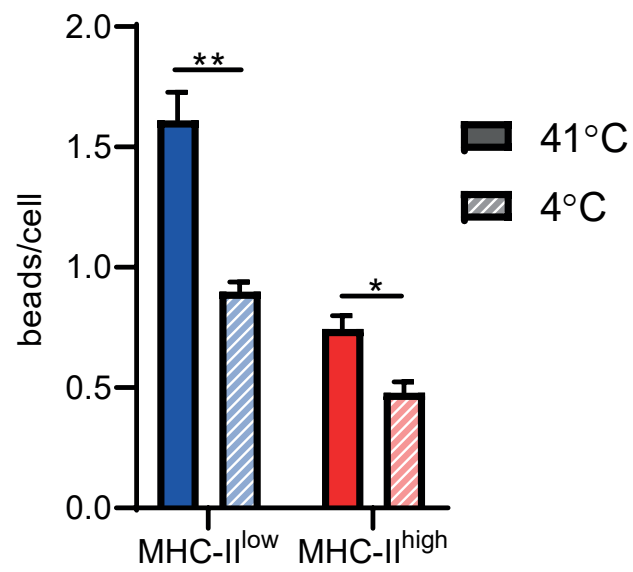

Supplementary Figure S3. Increased phagocytosis of IgY-opsonized beads by chBMDCs upon incubation at $41^{\circ} \mathrm{C}$ compared to $4^{\circ} \mathrm{C}$. IgY-opsonized beads were added to chBMDCs, followed by an incubation period of $4 \mathrm{~h}$ at either $4 \mathrm{I}^{\circ} \mathrm{C}$ or $4{ }^{\circ} \mathrm{C}$. Next, bead content of MHC-IIlow and MHC-II high chBMDC subsets was quantified by flow cytometry. The error bars show the SEM of an experiment performed in triplo. Unpaired t-tests were performed to test for statistically significant differences between the experiments performed at $4 \mathrm{I}{ }^{\circ} \mathrm{C}$ or $4{ }^{\circ} \mathrm{C}$ for both chBMDC subsets. A statistically significant difference between the experiments is shown by $*$ for $p<0.05$ and $* *$ for $p<0.01$.

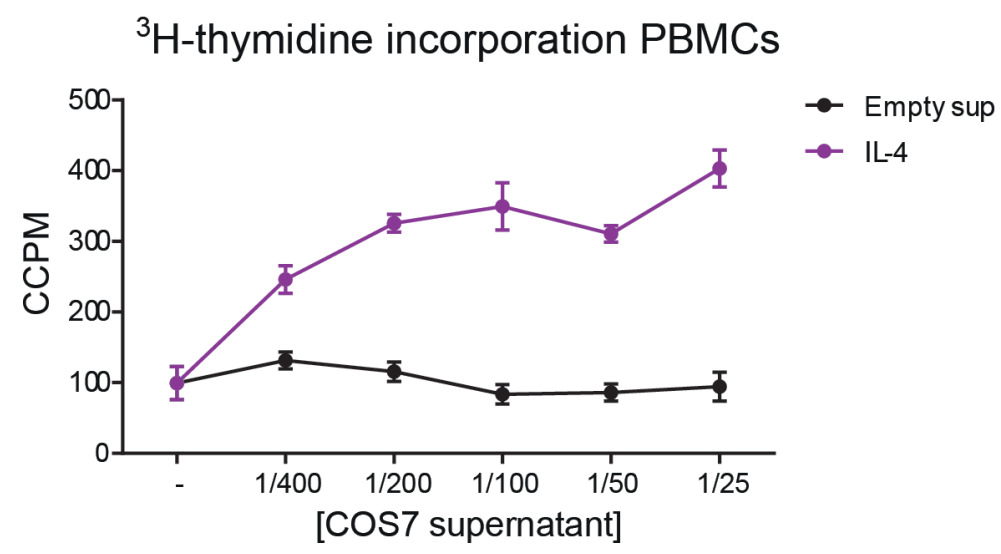

Supplementary Figure S4. IL-4 induced PBMC proliferation. PBMC proliferation was assessed by ${ }^{3} \mathrm{H}$-thymidine incorporation over a period of $18 \mathrm{~h}$ after 4 days of stimulation with COS-7 supernatant containing recombinant chicken IL-4 (purple) or empty COS-7 supernatant as a control (black) $(n=4)$. 

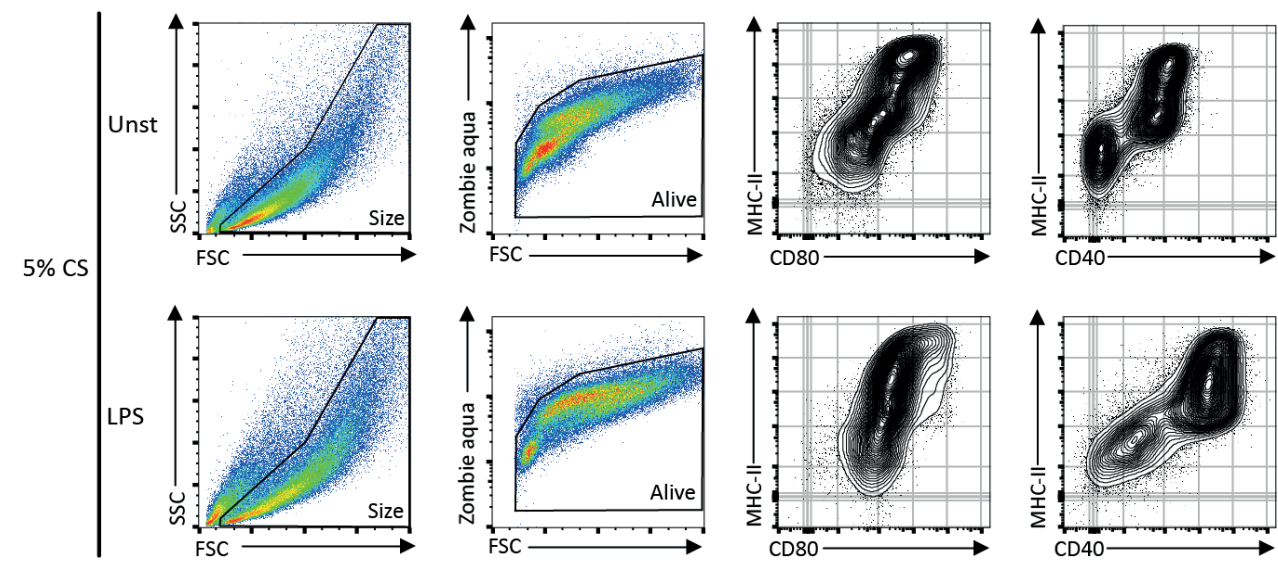

$10 \%$ FBS
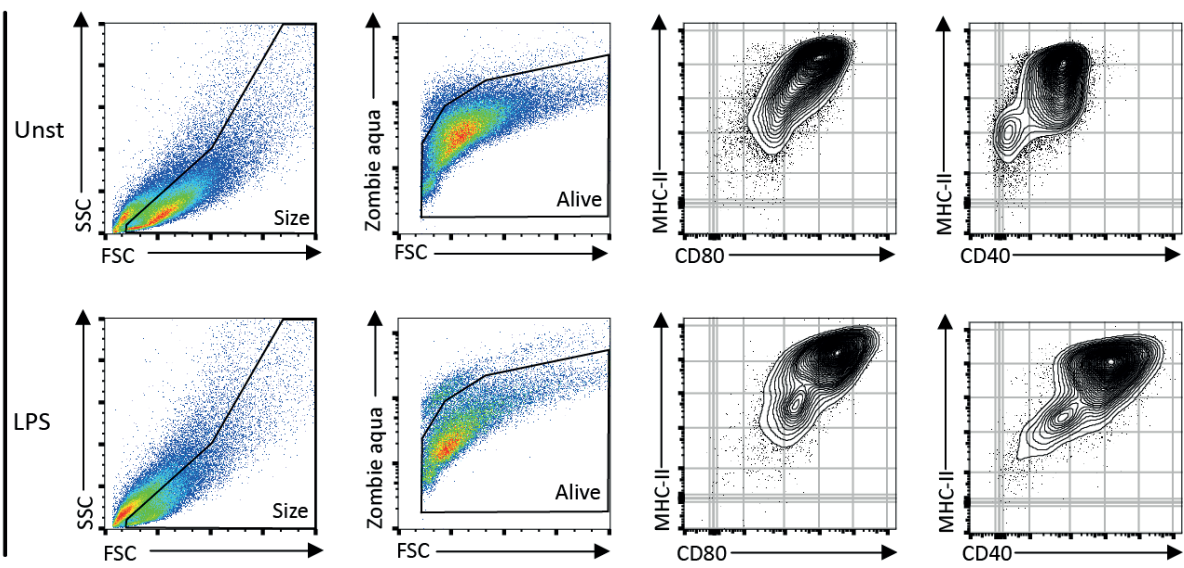

Supplementary Figure S5. The phenotypical outcome of chBMDCs is affected by serum supplements in the cell culture medium. 10\% FBS supplemented cell culture medium led to a more homogeneous chBMDC population with higher expression of MHC-II and CD80 compared to chBMDCs grown in cell culture medium supplemented with $5 \%$ chicken serum. The $\mathrm{FSC}^{\text {low }}$ and $\mathrm{MHC}$-Illow subsets from the chBMDC culture were almost absent in the adapted protocol that includes FBS supplementation. Moreover, LPS stimulation of chBMDCs in the presence of 10\% FBS led to an increase in both CD40 and CD80 expression, whereas only CD40 expression was found to be increased after stimulation of chBMDCs that were cultured according to the standard protocol that includes $5 \%$ chicken serum. 
Supplementary Table SI. The quantification of flow cytometry data. The average geometric mean fluorescent intensity (gMFI) of three independent replicates is shown for each marker for the MHCIIlow and MHC-II high chBMDC subsets. In addition, the ratio between the gMFIs of both subsets are shown for each marker.

\begin{tabular}{|c|c|c|c|}
\hline \multirow[t]{2}{*}{ Marker } & \multicolumn{2}{|c|}{ gMFI values per subset } & \multirow[t]{2}{*}{ Ratio $\mathrm{MHClI}$ low $/ \mathrm{MHCl}{ }^{\text {high }}$} \\
\hline & MHCIIlow & MHChigh & \\
\hline $\mathrm{CDI} I \mathrm{~b} / \mathrm{c}$ & $7.1 \times 10^{5}$ & $3.1 \times 10^{5}$ & 2.31 \\
\hline CD40 & $1.4 \times 10^{4}$ & $2.3 \times 10^{4}$ & 0.60 \\
\hline CD80 & $2.9 \times 10^{4}$ & $1.1 \times 10^{5}$ & 0.27 \\
\hline CSFIR & $1.4 \times 10^{5}$ & $4.5 \times 10^{4}$ & 3.05 \\
\hline c-Kit & $3.1 \times 10^{4}$ & $1.8 \times 10^{4}$ & 1.76 \\
\hline MRCIL-B & $1.8 \times 10^{5}$ & $2.4 \times 10^{5}$ & 0.74 \\
\hline CDI.I & $7.8 \times 10^{3}$ & $1.6 \times 10^{4}$ & 0.50 \\
\hline$\beta 2 m$ & $8.6 \times 10^{5}$ & $5.6 \times 10^{5}$ & 1.55 \\
\hline
\end{tabular}

Supplementary Table S2. The quantification of RT-qPCR data. The average 40-Ct-value of three independent replicates is shown for each marker for the MHC-Illow and MHC-Iligh chBMDC subsets. In addition, the difference between the gMFls of both subsets are shown for each marker.

\begin{tabular}{|c|c|c|c|}
\hline \multirow[t]{2}{*}{ Marker } & \multicolumn{2}{|c|}{$40-C t$ values per subset } & \multirow[t]{2}{*}{$\triangle \mathrm{MHCII}^{\text {low }}-\mathrm{MHCI}^{\text {high }}$} \\
\hline & MHCIIow & MHChigh & \\
\hline MerTK & 15.85 & 16.30 & -0.46 \\
\hline CDI4 & 8.82 & 8.82 & 0.00 \\
\hline TLR4 & 11.92 & 12.34 & -0.42 \\
\hline iNOS & 12.16 & 11.26 & 0.90 \\
\hline Zbtb46 & 14.02 & 15.50 & -1.48 \\
\hline CCR6 & 5.78 & 6.36 & -0.58 \\
\hline DEC205 & 12.78 & 12.47 & 0.31 \\
\hline DC-SIGN & 4.73 & 3.41 & 1.32 \\
\hline CCR7 & 1.30 & 6.64 & -5.35 \\
\hline CD83 & 11.93 & 14.90 & -2.98 \\
\hline
\end{tabular}

Supplementary Video SI. This shows a 3D constructed model created by confocal microscopy from chBMDCs that phagocytosed fluorescent beads (in red) for $4 \mathrm{~h}$. The cells from the chBMDC culture were stained for MHC-II (in blue) and the plasma membrane by using WGA (in green). This movie is available online at https://doi.org//0.3389/fimmu.2020.00141. 


\section{References}

I. Banchereau J, Steinman RM. Dendritic cells and the control of immunity. Nature (1998) 392(6673):245-252. doi: 10.1038/32588

2. Joffre $O$, Nolte MA, Spörri R, Reis e Sousa C. Inflammatory signals in dendritic cell activation and the induction of adaptive immunity. Immunol Rev (2009) 227(I):234-247. doi: 10.1 I I I/j. I600065X.2008.007/8.X

3. Gallucci S, Lolkema M, Matzinger P. Natural adjuvants: Endogenous activators of dendritic cells. Nat Med (1999) 5(II):1249-1255. doi:10.1038/15200

4. Medzhitov R, Janeway CA. Innate immunity: impact on the adaptive immune response. Curr Opin Immunol (I997) 9(I):4-9. doi: I0.1016/S0952-79I5(97)80I52-5

5. Inaba K, Inaba M, Romani N, Aya H, Deguchi M, Ikehara S, et al. Generation of large numbers of dendritic cells from mouse bone marrow cultures supplemented with granulocyte/macrophage colony-stimulating factor. J Exp Med (1992) I76(6): I693-1702. doi:10.1084/JEM.176.6.1693

6. dos Santos GG, Reinders J, Ouwehand K, Rustemeyer T, Scheper RJ, Gibbs S. Progress on the development of human in vitro dendritic cell based assays for assessment of the sensitizing potential of a compound. Toxicol Appl Pharmacol (2009) 236(3):372-382. doi:10.1016/j.taap.2009.02.004

7. Vandebriel R, Hoefnagel MMN. Dendritic cell-based in vitro assays for vaccine immunogenicity. Hum Vaccin Immunother (20I2) 8(9):I323-I325. doi:I0.4I6I/hv.2I350

8. Battais F, Huppert C, Langonné I, Muller S, Sponne I. In vitro detection of chemical allergens: an optimized assay using mouse bone marrow-derived dendritic cells. Contact Dermatitis (2017) 77(5):3 I I-322. doi: 10.1 I I I/cod. 12829

9. Ricklin Gutzwiller ME, Moulin HR, Zurbriggen A, Roosje P, Summerfield A. Comparative analysis of canine monocyte- and bone-marrow-derived dendritic cells. Vet Res (20I0) 4I(4):40. doi:I0.105I/vetres/2010012

10. Bienzle D, Reggeti F, Clark ME, Chow C. Immunophenotype and functional properties of feline dendritic cells derived from blood and bone marrow. Vet Immunol Immunopathol (2003) 96(I2):19-30. doi:10.1016/S0165-2427(03)00132-6

II. Hope JC, Werling D, Collins RA, Mertens B, Howard CJ. Flt-3 ligand, in combination with bovine granulocyte-macrophage colony- stimulating factor and interleukin-4, promotes the growth of bovine bone marrow derived dendritic cells. Scand J Immunol (2000) 5I(I):60-66. doi:10.1046/ j.1365-3083.2000.00646.x

12. Foulon E, Foucras G. Two populations of ovine bone marrow-derived dendritic cells can be generated with recombinant GM-CSF and separated on CDI Ib expression. J Immunol Methods (2008) 339(I):I-10. doi:I0.1016/J.JIM.2008.07.012

13. Carrasco CP, Rigden RC, Schaffner R, Gerber H, Neuhaus V, Inumaru S, et al. Porcine dendritic cells generated in vitro: morphological, phenotypic and functional properties. Immunology (200I) 104(2): 175-184. doi:I0.1046/j.1365-2567.200I.01299.x

14. Wu Z, Rothwell L, Young JR, Kaufman J, Butter C, Kaiser P. Generation and characterization of chicken bone marrow-derived dendritic cells. Immunology (2010) 129(I):133-145. doi:10.1 III/ j.1365-2567.2009.03129.x

15. de Geus ED, Tefsen B, van Haarlem DA, van Eden W, van Die I, Vervelde L. Glycans from avian influenza virus are recognized by chicken dendritic cells and are targets for the humoral immune response in chicken. Mol Immunol (2013) 56(4):452-462. doi:I0.10I6/j.molimm.2013.06.007

16. Vervelde L, Reemers SS, van Haarlem DA, Post J, Claassen E, Rebel JMJ, et al. Chicken dendritic cells are susceptible to highly pathogenic avian influenza viruses which induce strong cytokine responses. Dev Comp Immunol (2013) 39(3):198-206. doi:I0.1016/j.dci.2012.10.01 I

17. Liang J, Yin Y, Qin T, Yang Q. Chicken bone marrow-derived dendritic cells maturation in response to infectious bursal disease virus. Vet Immunol Immunopathol (2015) I64:5I-55. doi:10.1016/j.vetimm.2014.12.012 
18. Kamble NM, Jawale CV, Lee JH. Activation of chicken bone marrow-derived dendritic cells induced by a Salmonella Enteritidis ghost vaccine candidate. Poult Sci (2016) 95(10):2274-2280. doi: $10.3382 / \mathrm{ps} /$ pew 158

19. Kamble NM, Jawale CV, Lee JH. Interaction of a live attenuated Salmonella Gallinarum vaccine candidate with chicken bone marrow-derived dendritic cells. Avian Pathol (2016) 45(2):235-243. doi:10.1080/03079457.2016.1144919

20. Helft J, Böttcher J, Chakravarty P, Zelenay S, Huotari J, Schraml BU, et al. GM-CSF Mouse Bone Marrow Cultures Comprise a Heterogeneous Population of $\mathrm{CDI} \mathrm{Ic} \mathrm{MHCl}^{+} \mathrm{Macrophages} \mathrm{and}^{+}$ Dendritic Cells. Immunity (20I5) 42(6):I I97-I2II. doi:I0.10I6/j.immuni.20I5.05.0I8

21. Lutz MB, Inaba K, Schuler G, Romani N. Still Alive and Kicking: In-Vitro-Generated GM-CSF Dendritic Cells! Immunity (2016) 44(I):I-2. doi:I0.10I6/j.immuni.20I5.12.0I3

22. Guilliams M, Malissen B. A Death Notice for In-Vitro-Generated GM-CSF Dendritic Cells? Immunity (20I5) 42(6):988-990. doi:I0.1016/j.immuni.20I5.05.020

23. Guilliams M, Dutertre CA, Scott CL, McGovern N, Sichien D, Chakarov S, et al. Unsupervised High-Dimensional Analysis Aligns Dendritic Cells across Tissues and Species. Immunity (2016) 45(3):669-684. doi:10.10I6/j.immuni.2016.08.015

24. Guilliams M, Ginhoux F, Jakubzick C, Naik SH, Onai N, Schraml BU, et al. Dendritic cells, monocytes and macrophages: a unified nomenclature based on ontogeny. Nat Rev Immunol (20I4) I4(8):57I-578. doi:I0.1038/nri37/2

25. Helft J, Böttcher JP, Chakravarty P, Zelenay S, Huotari J, Schraml BU, et al. Alive but Confused: Heterogeneity of $\mathrm{CDIIc^{+ }}$ MHC Class $\mathrm{II}^{+}$Cells in GM-CSF Mouse Bone Marrow Cultures. Immunity (2016) 44(I):3-4. doi:I0.1016/J.IMMUNI.20I5.12.0I4

26. de Geus ED, Jansen CA, Vervelde L. Uptake of particulate antigens in a nonmammalian lung: phenotypic and functional characterization of avian respiratory phagocytes using bacterial or viral antigens. J Immunol (20I2) I88(9):45 I6-4526. doi:I0.4049/jimmunol. I 200092

27. Avery S, Rothwell L, Degen WDJ, Schijns VEJC, Young J, Kaufman J, et al. Characterization of the First Nonmammalian T2 Cytokine Gene Cluster: The Cluster Contains Functional Single-Copy Genes for IL-3, IL-4, IL-I3, and GM-CSF, a Gene for IL-5 That Appears to Be a Pseudogene, and a Gene Encoding Another Cytokinelike Transcript, KK34. J Interf Cytokine Res (2004) 24(I0):600610. doi:10.1089/jir.2004.24.600

28. Schindelin J, Arganda-Carreras I, Frise E, Kaynig V, Longair M, Pietzsch T, et al. Fiji: an opensource platform for biological-image analysis. Nat Methods (2012) 9(7):676-682. doi:10.1038/ nmeth.2019

29. Eldaghayes I, Rothwell L, Williams A, Withers D, Balu S, Davison F, et al. Infectious Bursal Disease Virus: Strains That Differ in Virulence Differentially Modulate the Innate Immune Response to Infection in the Chicken Bursa. Viral Immunol (2006) I9(I):83-9I. doi:10.1089/vim.2006.19.83

30. Wu Z, Hu T, Kaiser P. Chicken CCR6 and CCR7 are markers for immature and mature dendritic cells respectively. Dev Comp Immunol (20II) 35(5):563-567. doi:I0.10I6/J.DCI.2010.12.0I5

31. Guilliams M, Malissen B. A Matter of Perspective: Moving from a Pre-omic to a Systems-Biology Vantage of Monocyte-Derived Cell Function and Nomenclature. Immunity (2016) 44(I):5-6. doi:10.1016/J.IMMUNI.2015.12.020

32. Lutz MB, Kukutsch N, Ogilvie ALJ, Rößner S, Koch F, Romani N, et al. An advanced culture method for generating large quantities of highly pure dendritic cells from mouse bone marrow. J Immunol Methods (1999) 223(I):77-92. doi:I0.1016/S0022-I759(98)00204-X

33. Na YR, Jung D, Gu GJ, Seok SH. GM-CSF Grown Bone Marrow Derived Cells Are Composed of Phenotypically Different Dendritic Cells and Macrophages. Mol Cells (2016) 39(I0):734-74I. doi:10.14348/molcells.2016.0160

34. Guzman E, Pujol M, Ribeca P, Montoya M. Bovine Derived in vitro Cultures Generate Heterogeneous Populations of Antigen Presenting Cells. Front Immunol (2019) 10:612. doi:10.3389/fimmu.2019.00612 
35. Shalek AK, Satija R, Adiconis X, Gertner RS, Gaublomme JT, Raychowdhury R, et al. Single-cell transcriptomics reveals bimodality in expression and splicing in immune cells. 20I3. doi:10.1038/ nature 12172

36. Yasmin AR, Yeap SK, Tan SW, Hair-Bejo M, Fakurazi S, Kaiser P, et al. In vitro characterization of chicken bone marrow-derived dendritic cells following infection with very virulent infectious bursal disease virus. Avian Pathol (20I5) 44(6):452-462. doi: I0.1080/03079457.20I5.1084997

37. Cao X, Sugita M, Van Der Wel N, Lai J, Rogers RA, Peters PJ, et al. CDI molecules efficiently present antigen in immature dendritic cells and traffic independently of $\mathrm{MHC}$ class II during dendritic cell maturation. J Immunol (2002) I69(9):4770-4777. doi:I0.4049/jimmunol. I69.9.4770

38. Jiang A, Bloom O, Ono S, Cui W, Unternaehrer J, Jiang S, et al. Disruption of E-Cadherin-Mediated Adhesion Induces a Functionally Distinct Pathway of Dendritic Cell Maturation. Immunity (2007) 27(4):610-624. doi:10.1016/J.IMMUNI.2007.08.015

39. Satpathy AT, KC W, Albring JC, Edelson BT, Kretzer NM, Bhattacharya D, et al. Zbtb46 expression distinguishes classical dendritic cells and their committed progenitors from other immune lineages. J Exp Med (20I2) 209(6): I I35-I I 52. doi: I0. I084/jem.20I 20030

40. Sennikov S V, Falaleeva SA, Shkaruba NS, Chumasova OA, Obleukhova IA, Sizikov AE, et al. Maturation and cytokine production potential of dendritic cells isolated from rheumatoid arthritis patients peripheral blood and induced in vitro. Hum Immunol (2016) 77(10):930-936. doi:10.1016/j.humimm.2016.07.005

4I. Geijtenbeek TBH, Torensma R, van Vliet SJ, van Duijnhoven GC, Adema GJ, van Kooyk Y, et al. Identification of DC-SIGN, a Novel Dendritic Cell-Specific ICAM-3 Receptor that Supports Primary Immune Responses. Cell (2000) I00(5):575-585. doi:I 0. I0I6/S0092-8674(00)80693-5

42. Lutz MB, Schuler G. Immature, semi-mature and fully mature dendritic cells: Which signals induce tolerance or immunity? Trends Immunol (2002) 23(9):445-449. doi:10.1016/SI47I4906(02)0228I-0

43. Dudek AM, Martin S, Garg AD, Agostinis P. Immature, semi-mature, and fully mature dendritic cells: Toward a DC-cancer cells interface that augments anticancer immunity. Front Immunol (20I3) 4(DEC). doi:I0.3389/fimmu.2013.00438

44. Wu Z, Kaiser P. Antigen presenting cells in a non-mammalian model system, the chicken. Immunobiology (201 I) 216(II):II77-II83. doi:10.1016/j.imbio.20II.05.012

45. Platt CD, Ma JK, Chalouni C, Ebersold M, Bou-Reslan H, Carano RAD, et al. Mature dendritic cells use endocytic receptors to capture and present antigens. Proc Natl Acad Sci U S A (2010) 107(9):4287-4292. doi:10.1073/pnas.0910609107

46. Menges M, Baumeister T, Rössner S, Stoitzner P, Romani N, Gessner A, et al. IL-4 supports the generation of a dendritic cell subset from murine bone marrow with altered endocytosis capacity. J Leukoc Biol (2005) 77(4):535-543. doi: I0.I I89/jlb.0804473

47. Wang S, Sun X, Zhou H, Zhu Z, Zhao W, Zhu C. Interleukin-4 affects the mature phenotype and function of rat bone marrow-derived dendritic cells. Mol Med Rep (2015) 12(1):233-237. doi: $10.3892 / \mathrm{mmr} .2015 .3349$ 


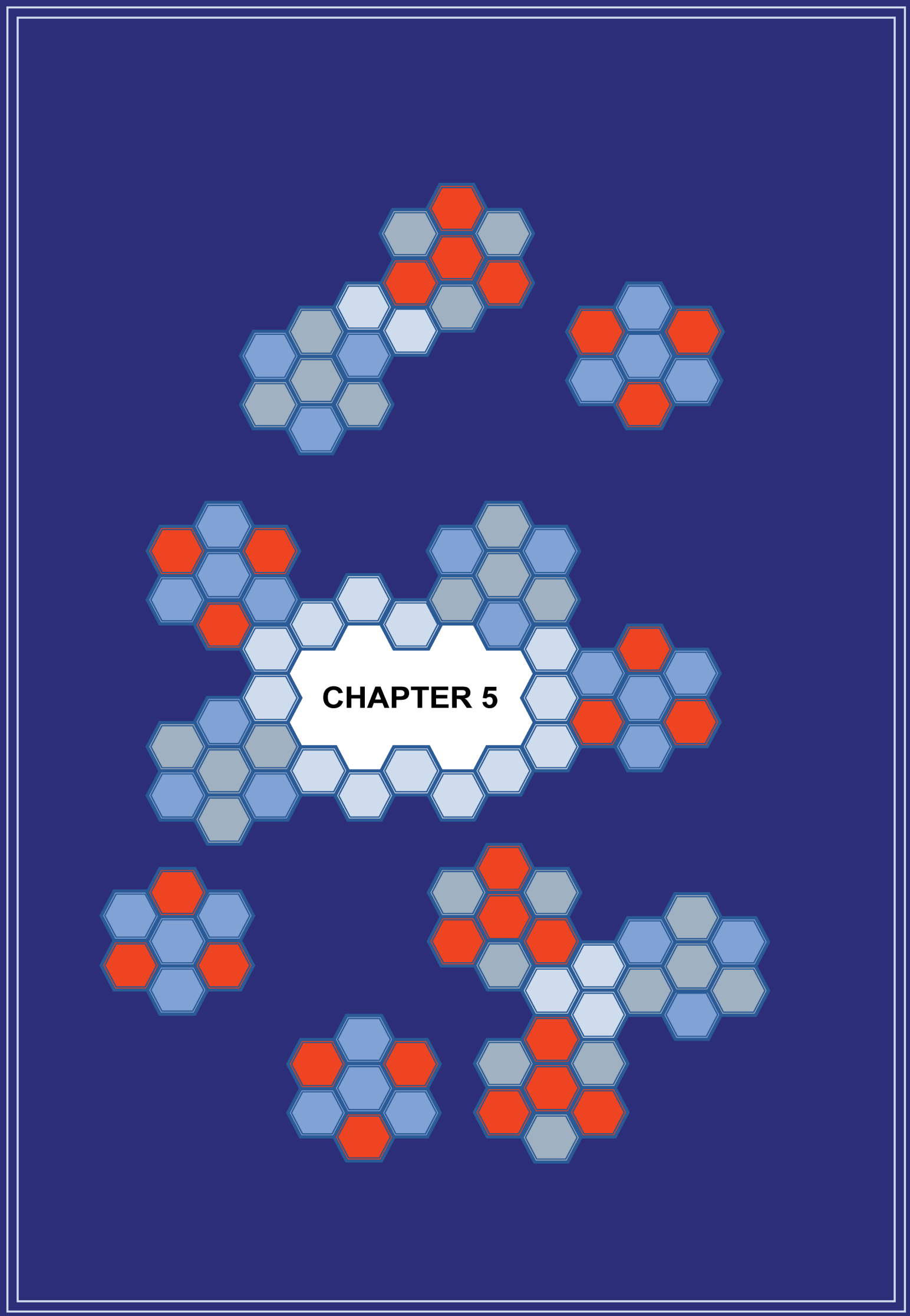




\section{Proteomic analysis of chicken bone marrow-derived dendritic cells in response to an inactivated IBV+NDV poultry vaccine}

Robin H.G.A. van den Biggelaar', Larissa van der Maas², Hugo D. Meiring², Jeroen L.A Pennings ${ }^{3}$, Willem van Eden', Victor P.M.G. Rutten ${ }^{1,4}$ and Christine A. Jansen'

' Division of Infectious Diseases and Immunology, Department of Biomolecular Health Sciences, Faculty of Veterinary Medicine, Utrecht University, Utrecht, the Netherlands

${ }^{2}$ Intravacc (Institute for Translational Vaccinology), the Netherlands

${ }^{3}$ Centre for Health Protection, National Institute for Public Health and the Environment (RIVM), Bilthoven, the Netherlands

${ }^{4}$ Department of Veterinary Tropical Diseases, Faculty of Veterinary

Science, University of Pretoria, Pretoria, South Africa

(Submitted) 


\section{Abstract}

Inactivated poultry vaccines are subject to routine potency testing for batch release, requiring large numbers of animals. The replacement of in vivo tests for cell-based alternatives can be facilitated by the identification of biomarkers for vaccine-induced immune responses. In this study, chicken bone marrow-derived dendritic cells were stimulated with an inactivated vaccine for infectious bronchitis virus and Newcastle disease virus, as well as inactivated infectious bronchitis virus only, and lipopolysaccharides as controls. Next, the cells were lysed and subjected to proteomic analysis. Stimulation with the vaccine resulted in 66 differentially expressed proteins associated with mRNA translation, immune responses, lipid metabolism and the proteasome. For the eight most significantly upregulated proteins, mRNA expression levels were assessed. Markers that showed increased expression at both mRNA and protein levels included PLIN2 and PSMBI. Stimulation with infectious bronchitis virus only resulted in 25 differentially expressed proteins, which were mostly proteins containing Src homology 2 domains. Stimulation with lipopolysaccharides resulted in II8 differentially expressed proteins associated with dendritic cell maturation and antimicrobial activity. This study provides leads to a better understanding of the activation of dendritic cells by an inactivated poultry vaccine, and identified PLIN2 and PSMBI as potential biomarkers for cell-based potency testing. 


\section{Introduction}

The safety and potency of poultry vaccines has traditionally been assessed through in vivo vaccination and challenge tests [I]. However, there is a global intent to replace in vivo vaccine tests for in vitro alternatives [2]. This has already led to the development of an enzyme-linked immunosorbent assay (ELISA) as an in vitro antigen quantification method to assess inactivated vaccines for Newcastle disease virus for potency [3]. However, other inactivated poultry vaccines still require in vivo tests to prove their potency and are thus in need for in vitro alternatives. The development of in vitro tests for inactivated poultry vaccines can be facilitated by the identification of biomarkers that may be inherent to the vaccine, demonstrating a consistent product profile of different vaccine batches [4-6], or be part of the vaccine-induced immune response that correlates with protection [7-10]. In particular, dendritic cells are ideal study targets because of their central role in the initiation of immune responsiveness [ $\mathrm{II}$ ]. One approach to discover biomarkers of immune responsiveness to inactivated poultry vaccines is the investigation of in vitro vaccine-stimulated immune cells by proteomic analysis using liquid chromatography-tandem mass spectrometry (LC-MS/MS).

Both infectious bronchitis virus (IBV) and Newcastle disease virus (NDV) cause respiratory tract infections that can disseminate to other tissues, leading to reduced egg production and mortality in chickens $[12,13]$. In this study, primary chicken bone marrow-derived dendritic cells (chBMDCs), which have been characterized in previous studies [14,15], were stimulated with a commercially available inactivated poultry vaccine against IBV and NDV in a mineral oil adjuvant in a water-in-oil formulation, and analyzed by LC-MS/MS to evaluate changes in the proteome In addition, chBDMCs were stimulated with inactivated IBV antigens only to discriminate between the effects of a single inactivated antigen and a vaccine. Stimulation with E. coli lipopolysaccharide (LPS) was included in this study as a strong stimulator of innate immune cells to validate the methodology used. Previous studies have shown that LPS stimulates maturation of chicken DCs, including increased surface expression of MHC class II molecules and costimulatory molecules [14-19], decreased endocytosis and phagocytosis [14,15], increased expression of proinflammatory cytokines and cytokines that promote the differentiation of naïve helper $T$ cells $[14,17,19]$, and an increased capacity to stimulate naïve $T$ cells $[14,15,18]$. The primary aim of this study was to search for biomarkers of vaccine-stimulated dendritic cells to use as targets for in vitro cellbased quality assessment of an inactivated IBV + NDV poultry vaccine. Secondly, this study may generate new hypotheses about the mechanisms by which an inactivated viral poultry vaccine activates chicken DCs, as well as new insights in the cellular processes involved in chBMDCs maturation after stimulation with LPS.

\section{Materials and Methods}

\section{Generation and stimulation of chBMDCs}

Bone marrow was isolated from eighteen-day-old embryonated NOVOgen Brown eggs (Verbeek Broederij, Zeewolde, the Netherlands) as described previously [15]. Experiments with embryonated chicken eggs are not considered animal experiments according to European legislation for the protection of animals used for scientific purposes (Directive $2010 / 63 / E U)$. Briefly, the bone marrow cells were obtained from the tibiae and femurs 
of 200 chicken embryos, which were pooled and resuspended with $2.5-5 \times 10^{7}$ cells $/ \mathrm{ml}$ in Roswell Park Memorial Institute (RPMI)-I640 cell culture medium supplemented with GlutaMAXTM_I, phenol red, HEPES (RPMI-1640; Gibco ${ }^{\mathrm{TM}}$, Life Technologies Limited, Paisley, UK), 50\% chicken serum (Gibco ${ }^{\mathrm{TM}}$ ) and 10\% dimethyl sulfoxide (Honeywell, Bucharest, Romania). The cells were viably frozen with $2.5-5 \times 10^{7}$ cells per cryotube and stored at $-140^{\circ} \mathrm{C}$ until further use.

Frozen bone marrow cells were thawed and cultured in Costar ${ }^{\circledR}$ 24-well plates (Corning®, Corning B.V. Life Sciences, Amsterdam, the Netherlands) in I ml/well RPMI-I 640 cell culture medium supplemented with $5 \%$ chicken serum and $50 \mathrm{U} / \mathrm{ml}$ of penicillin-streptomycin (all from $\mathrm{Gibco}^{\mathrm{TM}}$ ) in the presence of $2 \mu \mathrm{l} / \mathrm{ml}$ recombinant GM-CSF at $4 \mathrm{I}{ }^{\circ} \mathrm{C}, 5 \% \mathrm{CO}_{2}$ [I5]. Recombinant GM-CSF was produced using COS-7 cells transfected with $\mathrm{pCl}$-neo (Promega Corporation, Madison, WI, USA) expressing the relevant cytokine gene, a kind gift from P. Kaiser and L. Rothwell (Roslin Institute, Edinburgh, UK). GM-CSF was used at the concentration $(2 \mu \mathrm{l} / \mathrm{ml})$ that, after titration, resulted in the highest percentage of $\mathrm{MHC}-\mathrm{Il}^{+}$ $\mathrm{CD} 40^{+} \mathrm{CD} 80^{+}$cells, as determined using flow cytometry analysis [15]. In the morning of day 3, culture medium with non-adherent cells was removed, and I ml of fresh RPMI-I640 medium with GM-CSF was added. Approximately 32 hours later ( \pm 2 hours), on day 4, an additional I ml RPMI- 1640 medium with GM-CSF was added to the cultures. At day 7, chBMDCs were stimulated with $30 \mu \mathrm{l} / \mathrm{ml}$ IBV + NDV vaccine, $10 \mu \mathrm{l} / \mathrm{ml}$ IBV antigens (both were kind gifts from Boehringer Ingelheim, Ingelheim am Rhein, Germany), or 100 $\mathrm{ng} / \mathrm{ml}$ of LPS from E. coli OI27:B8 (Sigma-Aldrich, Saint Louis, MO, USA) for $24 \mathrm{~h}$ or left untreated. To create sample volumes with sufficient protein or RNA content, six wells of a 24-well plate were used for each condition. After $24 \mathrm{~h}$, the cell culture supernatant with floating cells was first collected. Subsequently, the cultures were washed with Dulbecco's phosphate-buffered saline without calcium and magnesium (PBS; Lonza, Basel, Switzerland) and the non-adherent cells were collected. The remaining adherent cells were incubated in PBS supplemented with 5 mM UltraPure EDTA (Invitrogen ${ }^{\mathrm{TM}}$, Life Technologies Europe $\mathrm{BV}$, Bleiswijk, the Netherlands) for $10 \mathrm{~min}$ at room temperature and harvested. All cellcontaining fluids per culture condition (cell culture medium, PBS and PBS 5 mM EDTA) obtained during the harvest procedure were pooled for subsequent experiments. The samples were spun at $400 \times \mathrm{g}$ for $5 \mathrm{~min}$ and the cells were resuspended in $100 \mathrm{mM}$ phosphate buffer (Sigma-Aldrich) and stored at $-80^{\circ} \mathrm{C}$ for subsequent LC-MS/MS analysis or lysed in RLT buffer (Qiagen GmbH, Hilden, Deutschland) and stored at $-20^{\circ} \mathrm{C}$ for RNA isolation. The preparation of chBMDC samples, for both the LC-MS/MS and gene expression analysis, was performed three times independently.

\section{Protein isolation, digestion and labelling}

Frozen samples were thawed, and the cells were lysed in $100 \mathrm{mM}$ phosphate buffer with $0.1 \%$ (w/v) RapiGest SF Surfactant (Waters Corporation, Milford, MA, USA) by vortexing and incubation at $80^{\circ} \mathrm{C}$ for $30 \mathrm{~min}$. Next, the protein content was determined by the Pierce BCA assay (ThermoFisher Scientific, Waltham, MA, USA) according to the manufacturer's instructions. The proteins were digested with $0.05 \mathrm{mg} / \mathrm{ml}$ Lys-C (Roche, Merck, Kenilworth, $\mathrm{NJ}$, USA) at an enzyme:substrate ratio of $\mathrm{I}: 400$ for $4 \mathrm{~h}$ at $37^{\circ} \mathrm{C}$ followed by digestion with $0.2 \mathrm{mg} / \mathrm{ml}$ trypsin (Promega) overnight at $37^{\circ} \mathrm{C}$ at an enzyme:substrate ratio of 1:100. Next, aliquots from the digests were labelled with native formaldehyde $\left(\mathrm{CH}_{2} \mathrm{O}\right.$ with $\mathrm{M}$ $=30.03 \mathrm{~g} / \mathrm{mol}$, hereinafter referred to as "light") (Sigma-Aldrich) together with sodium 
cyanoborohydride $\left(\mathrm{NaCNBH}_{3}\right)$ (Sigma-Aldrich), both at final concentrations of $30 \mathrm{mM}$, and incubation for $2 \mathrm{~h}$ at $37^{\circ} \mathrm{C}$. In addition, an internal standard was created by pooling digest aliquots of all stimulated samples (LPS, IBV antigen or IBV + NDV vaccine) and corresponding unstimulated samples, each of equal peptide mass as determined before digestion. This socalled "Common Reference" was labelled with deuterated formaldehyde $\left(\mathrm{CD}_{2} \mathrm{O}\right.$ with $\mathrm{M}=$ $32.04 \mathrm{~g} / \mathrm{mol}$, hereinafter referred to as "heavy") (Sigma-Aldrich), together with $\mathrm{NaCNBH}_{3}$, both at final concentrations of $30 \mathrm{mM}$, and incubation for $2 \mathrm{~h}$ at $37^{\circ} \mathrm{C}$.

\section{LC-MS/MS analysis and data processing}

Each "light"-labelled sample was mixed with an aliquot of the "heavy"-labelled Common Reference in a I:I ratio (based on the mass of the peptides). The resulting mixtures were desalted on a GX 27I Automated Solid Phase Extraction system (Gilson International B.V., The Hague, The Netherlands). Briefly, each mixture was loaded onto a I ml capacity CI8 cartridge (Waters) and the absorbed peptides were washed with $600 \mu \mathrm{l}$ of water containing 0.1 vol\% of formic acid and subsequently eluted with $600 \mu \mathrm{l}$ of 60 vol\% of acetonitrile in water, also containing 0.1 vol\% of formic acid. Each eluate was dried by centrifugation under reduced pressure (Eppendorf Vacuum Concentrator Plus, Eppendorf, Germany) and reconstituted in $100 \mu \mathrm{l}$ of water containing 5 vol\% of dimethyl sulfoxide and 0.1 vol\% of formic acid for nanoscale LC MS/MS analysis.

The nanoscale LC-MS system comprised an Agilent 1290 Infinity UPLC system (Agilent, Waldbronn, Germany) coupled to a Tribrid Orbitrap Fusion Lumos mass spectrometer (ThermoFisher Scientific). The system was setup essentially as described [20] containing an inhouse-made trapping column (20 mm length x $100 \mu \mathrm{m}$ I.D., Reprosil-Pur® CI8 AQ $5 \mu \mathrm{m}$ particle size, $120 \AA$ pore size, Dr. Maisch, Ammerbuch, Germany) and an analytical column (32.4 cm length $\times 50 \mu \mathrm{m}$ I.D., Reprosil-Pur ${ }^{\circledR}$ CI8-AQ $3 \mu \mathrm{m}$ particle size, $120 \AA$ pore size, dr. Maisch $\mathrm{GmbH}$ ) made in-house at Intravacc, the Netherlands. Solvent $A$ was 0.1 vol\% of formic acid in water and solvent B was 0.1 vol\% of formic acid in acetonitrile. The peptides were trapped on the trapping column for $10 \mathrm{~min}$ at a flow rate of $5 \mu \mathrm{l} / \mathrm{min}$ of $100 \%$ solvent A. The subsequent separation of the peptides was performed at a flow rate of $125 \mathrm{nl} / \mathrm{min}$ in a $95-\mathrm{min}$ non-linear gradient ( $8 \%$ solvent $B$ to $28 \%$ solvent $B$ in $0.25 \% / \mathrm{min}$, followed by an increase to $43 \%$ solvent B in $1.5 \% / \mathrm{min}$ and a step to $85 \%$ solvent B hold for $5 \mathrm{~min}$ ). The column effluent was electrosprayed into the MS with an inhouse-made gold/carboncoated fused silica spray tip (360 $\mu$ m O.D. x $25 \mu \mathrm{m}$ I.D., manually tapered to a $3.5-\mu \mathrm{m}$ tip I.D.), butt-connected to the analytical column. Full scan mass spectra were acquired with an Orbitrap readout at a $120,000 \mathrm{FWHM}$ resolution with an $\mathrm{m} / \mathrm{z}$ scan range of $300-1,500$ $\mathrm{Da}$, a maximum ion injection time of $50 \mathrm{~ms}$ and the Automatic Gain Control (AGC) set to 200,000 . Internal mass calibration with fluoranthene was enabled. CID fragmentation scans with an lon Trap readout were acquired for $z=I$ and $z=2-5$ positively charged ions with intensity thresholds of $1 \times 10^{6}$ or 5,000 counts, respectively. Dynamic exclusion was set to 2 for a $45-\mathrm{sec}$ time window. Default instrument settings were used for MS/MS acquisition.

Acquired data were processed with PEAKS® X (Bioinformatics Solutions Inc., Waterloo, Canada) against the Gallus gallus domesticus database (Taxonomy ID=903 I, www.uniprot. org) containing 34,930 entries for protein identification. Enzyme specificity was set to Trypsin (semispecific) with a maximum of 3 miscleavages. Mass error tolerances for parent ions and fragment ions were set to $20 \mathrm{ppm}$ and $0.6 \mathrm{Da}$, respectively. Fixed modifications 
were carbamidomethylating on Cys $(+57.0215 \mathrm{Da})$ and dimethylation on Lys $(+28.0313$ $\mathrm{Da}$ and $+32.0564 \mathrm{Da}$ for Light and Heavy, respectively). The variable modifications were deamidation on Asn and Gln (+0.9840 Da) and oxidation on Met (+ I5.9949 Da). Peak area intensities of the precursor ions of the identified peptides were used for relative protein quantification.

\section{Analysis of relative protein abundance}

Data processing and statistical analysis was done using $R$ statistical software, version 3.6.0. The relative quantification of the samples was based on the comparison of all samples against the Common References (Supplementary Table SI) acting as an internal standard between samples, in accordance with a previous publication [2I]. The resulting sample I Common Reference ratios were $\log _{2}$-transformed. Next, the values were normalized for variations between measurements by performing a median correction, in which all relative protein expression values were divided by median protein expression value of each run (Supplementary Table S2), in accordance with previous publications [22,23]. Proteins were included as quantitative differentially expressed proteins (DEPs) if they were detected in at least two out of three replicates for both stimulated and unstimulated samples, showed an average upregulation or downregulation of at least I.5-fold upon stimulation, and change was statistically significant $(p<0.05)$ according to a Student's T-test. Moreover, de novoexpressed proteins (i.e. uniquely expressed proteins detected in all samples of the stimulated group and in no samples of the unstimulated group, or the reverse situation) were also included as qualitative DEPs in the results. Although statistics could not be performed for these de novo-expressed proteins with expression levels were below the detection limit in one of the treatment groups, the qualitative differences were still considered to be of practical significance. Finally, some proteins appeared more than once in the results, while showing the same expression values. These redundant appearances were removed resulting in a list of unique proteins.

Volcano plots were made using Perseus software [24] to visualize the selection of DEPs. The selected DEPs were further analysed for protein-protein association networks using the STRING database (www.string-db.org) [25]. The database was searched for physical or functional protein-protein interactions with a minimum required interaction score of 0.700 (high confidence) among the DEPs. The resulting STRING network clusters were functionally classified using annotations with the lowest Benjamini-Hochberg false-discovery rates from integrated Reactome (www.reactome.org), KEGG (www.kegg.jp), GO (www.ebi. ac.uk/QuickGol), PFAM (www.pfam.xfam.org) and SMART (www.smart.embl-heidelberg. de) databases. Additionally, the STRING network was exported as tabular text output, and combined with protein expression data in Microsoft Excel. The resulting files were loaded in Cytoscape [26] version 3.7.2 for visualization.

The subcellular localization of DEPs was determined based on GO terms for cellular component using QuickGO (https://www.ebi.ac.uk/QuickGO/). The search was limited to a selected number of gene ontology terms for cellular components listed in Supplementary Table S3. The UniProtKB database was used as a reference for chicken proteins by choosing taxon identifier 903 I (Gallus gallus). The DEPs of stimulated chBMDCs were tested for enrichment for cellular components as compared to the reference using a one-tailed binominal test. Next, the Benjamini-Hochberg method was used to control false discovery 
rates (FDR) as a result of multiple comparisons [27]. An FDR $<0.05$ was considered significant.

To create KEGG pathway maps, the KEGG Mapper tool (http://www.genome.jp/kegg/ mapper.html) [28] was used to modify the maps gga03050 (Proteasome - Gallus gallus [chicken]), gga0 I 100 (Metabolic pathways - Gallus gallus [chicken]) and gga04I 42 (Lysosome - Gallus gallus [chicken]). Permission was granted by Kanehisa Laboratories to publish the KEGG pathway map images both in print and digital under the CC BY 4.0 open access license.

\section{Gene expression analysis}

RNA isolation was performed with the RNeasy Mini Kit (Qiagen) according to the manufacturer's instructions, including a DNase treatment step using the RNase-Free DNase Set (Qiagen). Next, cDNA was prepared using the reverse transcriptase from the iScript cDNA Synthesis Kit (Bio-Rad Laboratories B.V., Veenendaal, the Netherlands) according to the manufacturer's instructions. RT-qPCRs were performed for several genes of the most significantly upregulated DEPs with primers listed in Table I and SYBR Green Master Mix (both from Life Technologies). RT-qPCRs were performed with a CFX Connect and analyzed with the CFX Maestro software (both from Bio-Rad). All RT-qPCRs were evaluated for proper amplification efficiency (90-110\%) using serial dilutions of reference cDNA obtained from the chicken macrophage-like cell line HDII after $3 \mathrm{~h}$ stimulation with LPS. RT-qPCRs were performed in triplicate for every sample. Relative gene expression levels after stimulation of chBMDCs were normalized using the housekeeping gene GAPDH as the reference gene expressed as $\log _{2}$-transformed fold changes compared to unstimulated chBMDCs. Genes were considered significantly differentially expressed if at least two out of three stimulated replicates were upregulated or downregulated compared to unstimulated controls, the change was at least I.5-fold after stimulation, and the difference was statistically significant $(p<0.05)$ according to a Student's T-test.

\section{Results}

Differentially expressed proteins identified in chBMDCs stimulated with IBV + NDV vaccine did not show overlap with differentially expressed proteins after stimulation with IBV antigen only

Quantitative proteome analysis was performed for chBMDCs stimulated with a IBV + NDV vaccine, IBV antigen or LPS to identify the respective potential biomarkers. Initially, LC-MS/ MS led to the identification of 2,828 proteins (Supplementary Table SI), some of which were identified multiple times, because several UniProtKB accession codes were present for these individual protein sequences, and these were manually removed. The resulting list contained I 54 quantitative DEPs, which were found to be differentially expressed with statistical significance $(\geq 1.5$-fold with $p \leq 0.05)$ after stimulation with IBV + NDV vaccine, IBV antigen or LPS (Supplementary Tables S2 and S3). In addition, 45 proteins were differentially expressed at a qualitative level, i.e. these proteins were detected only in either unstimulated or any of the stimulated cultures of chBMDCs (Supplementary Tables S2 and S3). We found 33 upregulated and 34 downregulated DEPs after stimulation with the IBV + NDV vaccine (Figure I a). Less DEPs, I 4 upregulated and I I downregulated proteins, 
(a)

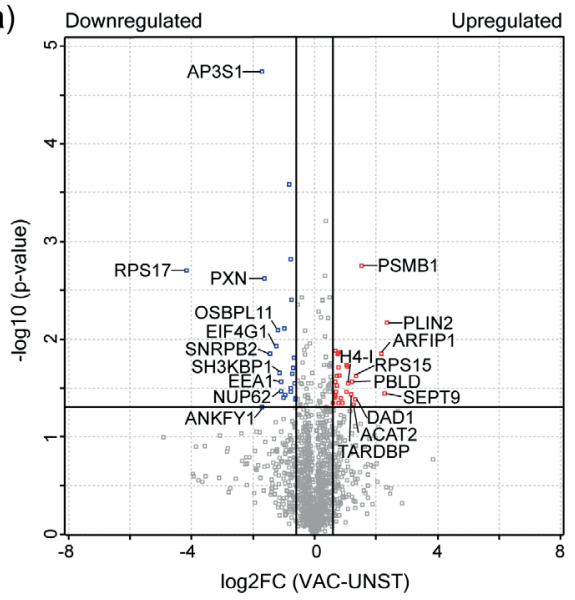

(c)

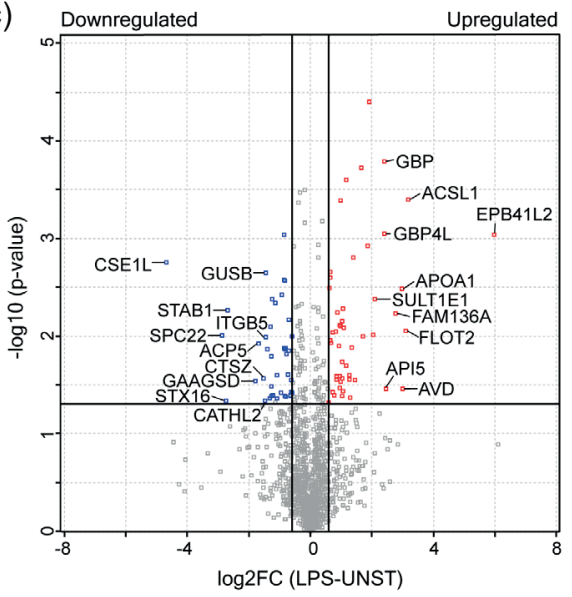

(b)

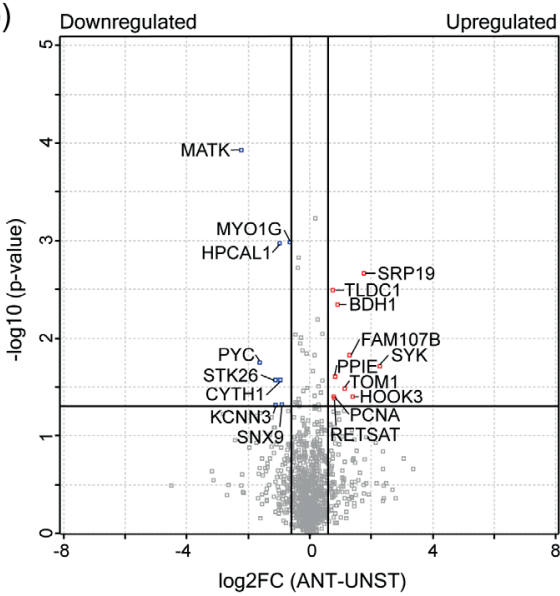

(d)

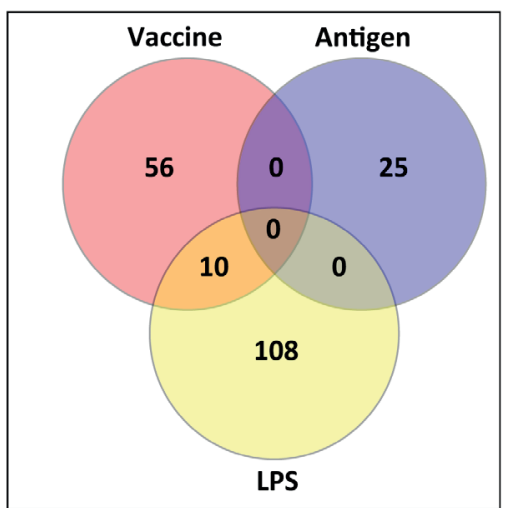

(k) Reference
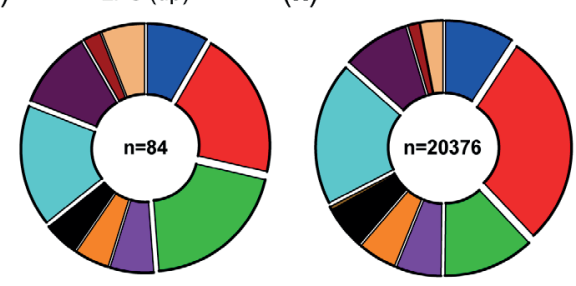

(f)

(h)

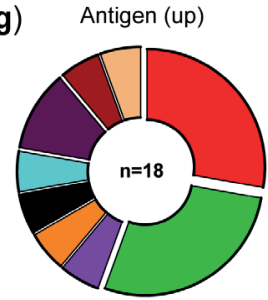

(i)

(j)

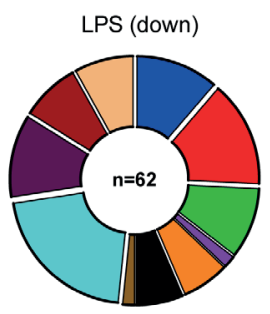

$\square$ Extracellular region

$\square$ Nucleus

$\square$ Cytosol

$\square$ Mitochondrium

口 Goldi apparatus

Endoplasmatic reticulum

$\square$ Peroxisome

$\square$ Plasma membrane

$\square$ Cytoskeleton

$\square$ Lysosome

$\square$ Endosome 
Figure I. Differentially expressed proteins identified by LC-MS/MS after stimulation of chBMDCs. Volcano plots show differentially expressed proteins of chBMDCs after stimulation with LPS (a), an IBV + NDV vaccine (b) and IBV antigens only (c). Proteins that are $\geq 1.5$-fold upregulated or downregulated and significantly different from the unstimulated controls are shown respectively in red and blue. The protein names are shown for up to 10 of the most upregulated and downregulated proteins for each condition. (d) A total of 199 proteins were found to be differentially expressed after the different stimulations. The overlap in differentially expressed proteins between stimulation conditions is given by a Venn diagram, which shows an overlap of 10 proteins between LPS- and vaccine-stimulated chBMDCs. The subcellular localization of differentially expressed proteins after stimulation with LPS (e and $\mathbf{f}), I B V+N D V$ vaccine $(\mathbf{g}$ and $\mathbf{h})$ and IBV antigen only (i and $\mathbf{j}$ ) was determined using gene ontology terms for cellular compartments (GOCC). A distinction was made between upregulated (e, $\mathbf{g}$ and $\mathbf{i})$ and downregulated $(\mathbf{f}, \mathbf{h}$ and $\mathbf{j})$ differentially expressed proteins. The GOCC terms of all proteins from the UniProtKB reference database are shown as well (k). Not all proteins could be annotated with gene ontology terms for cellular compartments and some proteins were annotated with multiple entries.

were found after stimulation with IBV antigen (Figure Ib). Stimulation with LPS induced a total of 66 upregulated and 52 downregulated DEPs (Figure Ic). The Venn diagram (Figure Id) shows overlaps of DEPs induced by the different stimuli. The overlap between DEPs from IBV + NDV vaccine- and LPS-stimulated chBMDCs comprised 10 proteins of which five showed a change into the same direction for both stimuli (Supplementary Table S4). No DEPs were shared between IBV + NDV vaccine and IBV antigen, or LPS and IBV antigen.

The DEPs after stimulation with IBV + NDV vaccine (Figures I e and $\mathbf{f}$ ), IBV antigen (Figures $\mathbf{I} \mathbf{g}$ and $\mathbf{h}$ ) or LPS (Figures $\mathbf{I} \mathbf{i}$ and $\mathbf{j}$ ) were annotated for subcellular localization using gene ontology terms for cellular compartments. The DEPs were distributed throughout all major cellular compartments. Overall, most DEPs were associated with the nucleus, plasma membrane and the cytosol, which is similar to the overall distribution of annotated proteins in the UniProtKB reference database (Figure I k). Compared to the reference, upregulated DEPs of vaccine-stimulated chBMDCs were relatively often associated with the extracellular region (FDR $=0.054)$ (Figure Ie), whereas downregulated DEPs were more associated with the cytosol $(F D R=0.059)$ (Figure If). Stimulation with IBV antigen resulted in a significant enrichment of upregulated DEPs associated with the cytosol (FDR $=0.046)$ and downregulated DEPs associated with the Golgi apparatus (FDR < 0.050) (Figures Ig and h). LPS stimulation of chBMDCs resulted in a significantly large proportion of upregulated DEPs associated with the cytosol (FDR $<0.001)$ and downregulated DEPs associated with the plasma membrane $(F D R=0.043)$, lysosome $(F D R=0.003)$ and endosome $(F D R=$ 0.037) (Figures I i and j).

\section{Differentially expressed proteins of vaccine-stimulated chBMDCs cluster in functional networks related to mRNA translation, immune responses, metabolism and the proteasome}

As a next step, an enrichment analysis of protein-protein interaction networks was performed using STRING. A STRING network was created using the DEPs found for the different stimuli. From the 199 DEPs that were included in the analysis we found 9I DEPs that formed 136 high confidence physical or functional protein-protein interactions (Figures 2, 5 and 6). These DEPs formed 16 clusters comprising 2 to 14 proteins (Supplementary Table S5). 
Chapter 5 | Proteomic analysis of chBMDCs stimulated with IBV + NDV vaccine

Table I. Primer sequences for RT-qPCR to assess gene expression of selected upregulated DEPs.

\begin{tabular}{|c|c|c|c|}
\hline Gene & Reference & Type & Sequences (5'-3') \\
\hline \multirow[t]{2}{*}{ PBLD } & \multirow[t]{2}{*}{ ENSGALT00000006268.6 } & Forward & CGGTTTGTCTGCTCGAAAATGAC \\
\hline & & Reverse & AACCATCGGAGTCCAAAGCG \\
\hline \multirow[t]{2}{*}{ ACAT2 } & \multirow[t]{2}{*}{ ENSGALT000000I9060.5 } & Forward & CCGGATCGTATCTTGGGCTCA \\
\hline & & Reverse & AGTCCAGCCGGCTTTCTCAATA \\
\hline \multirow[t]{2}{*}{ RPSI 5} & \multirow[t]{2}{*}{ ENSGALT00000070660.2 } & Forward & CCAAGATGGCAGAAGTGGAGCA \\
\hline & & Reverse & AGGACATATCGAGCAGCTGGT \\
\hline \multirow[t]{2}{*}{ DADI } & \multirow[t]{2}{*}{ ENSGALT00000048785.2 } & Forward & AGCTTCATCCTCGGCGTTTGT \\
\hline & & Reverse & CCCTCTCTGGTGAAATGCCTTGG \\
\hline \multirow[t]{2}{*}{ PSMBI } & \multirow[t]{2}{*}{ ENSGALT000000। 8217.5} & Forward & GCCATTTTGCCGTGAGACAGC \\
\hline & & Reverse & ACACAGTCCCGCCGTTGAAG \\
\hline \multirow[t]{2}{*}{ ARFIPI } & \multirow[t]{2}{*}{ ENSGALT00000065974.3 } & Forward & AGGTTCCTCAGCATCACCCA \\
\hline & & Reverse & ACCGGTCCACTCTTCTGCTG \\
\hline \multirow[t]{2}{*}{ SEPT9 } & \multirow[t]{2}{*}{ ENSGALT00000047767.2 } & Forward & GAAGGTCCAGAGGCCGTTCC \\
\hline & & Reverse & GCATCGATCCCCACGTAGCC \\
\hline \multirow[t]{2}{*}{ PLIN2 } & \multirow[t]{2}{*}{ ENSGALT00000083439.2 } & Forward & CCGCTCAACTGGCTGGTTCC \\
\hline & & Reverse & GGGCCATTTGTGCGTGAAAAGT \\
\hline \multirow[t]{2}{*}{ SRPI9 } & \multirow[t]{2}{*}{ ENSGALT00000000294.6 } & Forward & GGTAGGCCTGGTCGTTAGCA \\
\hline & & Reverse & AAATCTCTCCTTGTCGGCCGG \\
\hline \multirow[t]{2}{*}{ HOOK 3} & \multirow[t]{2}{*}{ ENSGALT00000024828.6 } & Forward & ACAACCATGAGATTCTAGGGCAAC \\
\hline & & Reverse & GCATTCTTCCAAGCTCCGCA \\
\hline \multirow[t]{2}{*}{ SYK } & \multirow[t]{2}{*}{ ENSGALT00000032807.5 } & Forward & CCCTCTGGCAGTTAGTTGAGCA \\
\hline & & Reverse & TCACTTTCTGAGCCATGCCGT \\
\hline \multirow[t]{2}{*}{ GBP4L } & \multirow[t]{2}{*}{ ENSGALT0000006956।.2 } & Forward & AGCGGCCCATCAGTGAGGAT \\
\hline & & Reverse & GAGCCGCGTCCCATTGTAGT \\
\hline \multirow[t]{2}{*}{ APOAI } & \multirow[t]{2}{*}{ ENSGALT000000 I I 524.6} & Forward & CTGTGCTCTTCCTGACGGG \\
\hline & & Reverse & GAGCCATGTCCTCACGCA \\
\hline \multirow[t]{2}{*}{ ACSLI } & \multirow[t]{2}{*}{ ENSGALT000000I 7294.4} & Forward & AGCCCAGGGAGAGTACATAGCA \\
\hline & & Reverse & AGGCCTGCAAGCTCTCTCCA \\
\hline \multirow[t]{2}{*}{ EPB4IL2 } & \multirow[t]{2}{*}{ XM_025I4893I.I } & Forward & CATCGGGCCGCTAAGAGGTT \\
\hline & & Reverse & TGTCGTGTCTGTGCTTGCGT \\
\hline \multirow[t]{2}{*}{ GAPDH } & ENSGALT00000023323.6 & Forward & GTGGTGCTAAGCGTGTTATC \\
\hline & & Reverse & GCATGGACAGTGGTCATAAG \\
\hline
\end{tabular}


The DEPs of IBV + NDV vaccine-stimulated chBMDCs were found overrepresented in clusters a (8//2), c (7//4), d (4/8) and h (3/6) (Figure 2). For cluster a, the top ranking functional enrichments were "mRNA translation" (Reactome: GGA-72766, FDR = I.6x 10-12; GO: $\left.0006412, F D R=2.3 \times 10^{-4}\right)$ representing all proteins except NME2, an enzyme required for synthesis of nucleoside triphosphates, and ETFI, which plays a role in the termination of translation. Cluster $\mathbf{a}$ is linked to cluster $\mathbf{b}$ through COPS4, a subunit of the COP9 signalosome complex. After stimulation with IBV + NDV vaccine, 3/7 proteins of cluster b were identified as DEPs. The highest ranking functional enrichments for cluster $\mathbf{b}$ were "DNA repair" (Reactome: GGA-73894, FDR = I.3×10-8), "transcription-coupled nucleotide excision repair [TC-NER]" (Reactome: GGA-678I827, FDR $=7.9 \times 10^{-7}$ ) and mRNA splicing (Reactome: GGA-72163, FDR $=6.5 \times 10^{-6}$ ), which together comprised all DEPs of this cluster. Cluster c was most functionally enriched for "immune system" (Reactome: GGAI68256; FDR $=4.5 \times 10^{-8}$ ), followed by the more specific description "innate immune system" (Reactome: GGA-I68249; FDR $=2.7 \times 10^{-7}$ ). Except for PXN, which is involved in focal adhesion, all DEPs found in cluster $\mathbf{c}$ after IBV + NDV vaccine stimulation were described to be involved in immune responses. Within cluster $\mathbf{d}$, two upregulated DEPs (OXCTI and ACAT2) are part of lipid metabolism and another two upregulated DEPs (SUCLG2 and IDH3G) are part of the citrate cycle (Figure 3). Cluster $\mathbf{f}$ was functionally enriched for "proteasome" (KEGG: gga03050; FDR $=6.4 \times 10^{-12}$ ) and comprised subunits of this protein complex that has a critical role in cellular proteolysis, including in the processing of antigens for presentation on the cell surface to the adaptive immune system. Proteins of cluster $\mathbf{f}$

(a)

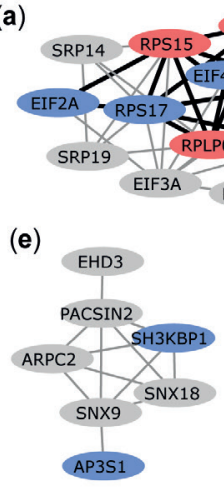

(i)

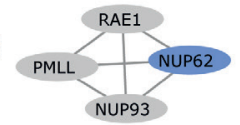

(j)

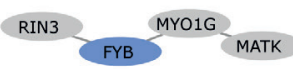

(f) (b)

(c)

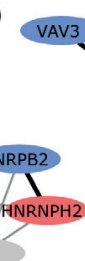

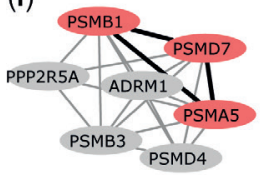

$(\mathbf{g})$
GALNS

(g)

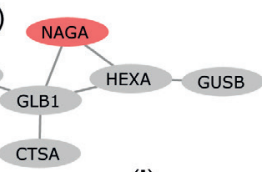

(h)

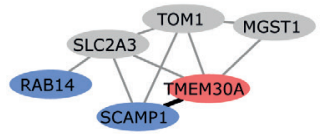

(I)

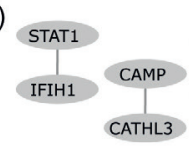

(d)

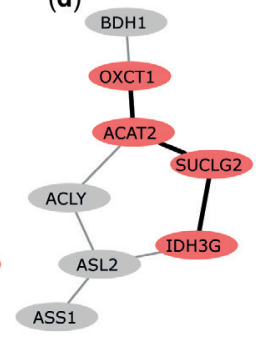

Figure 2. Cytoscape STRING network of differentially expressed proteins shows clusters of differentially expressed proteins after IBV + NDV vaccine stimulation. Upregulated differentially expressed proteins are shown in red and downregulated differentially expressed proteins are shown in blue. Proteins in grey were not significantly differentially expressed after IBV + NDV vaccine stimulation. Networks are only shown for proteins with a minimum required interaction score of 0.700 (high confidence). STRING interactions between proteins that are both differentially expressed by vaccine exposure are shown in black, otherwise in grey. The letters indicate different clusters with cluster I representing six clusters comprising two proteins each. 


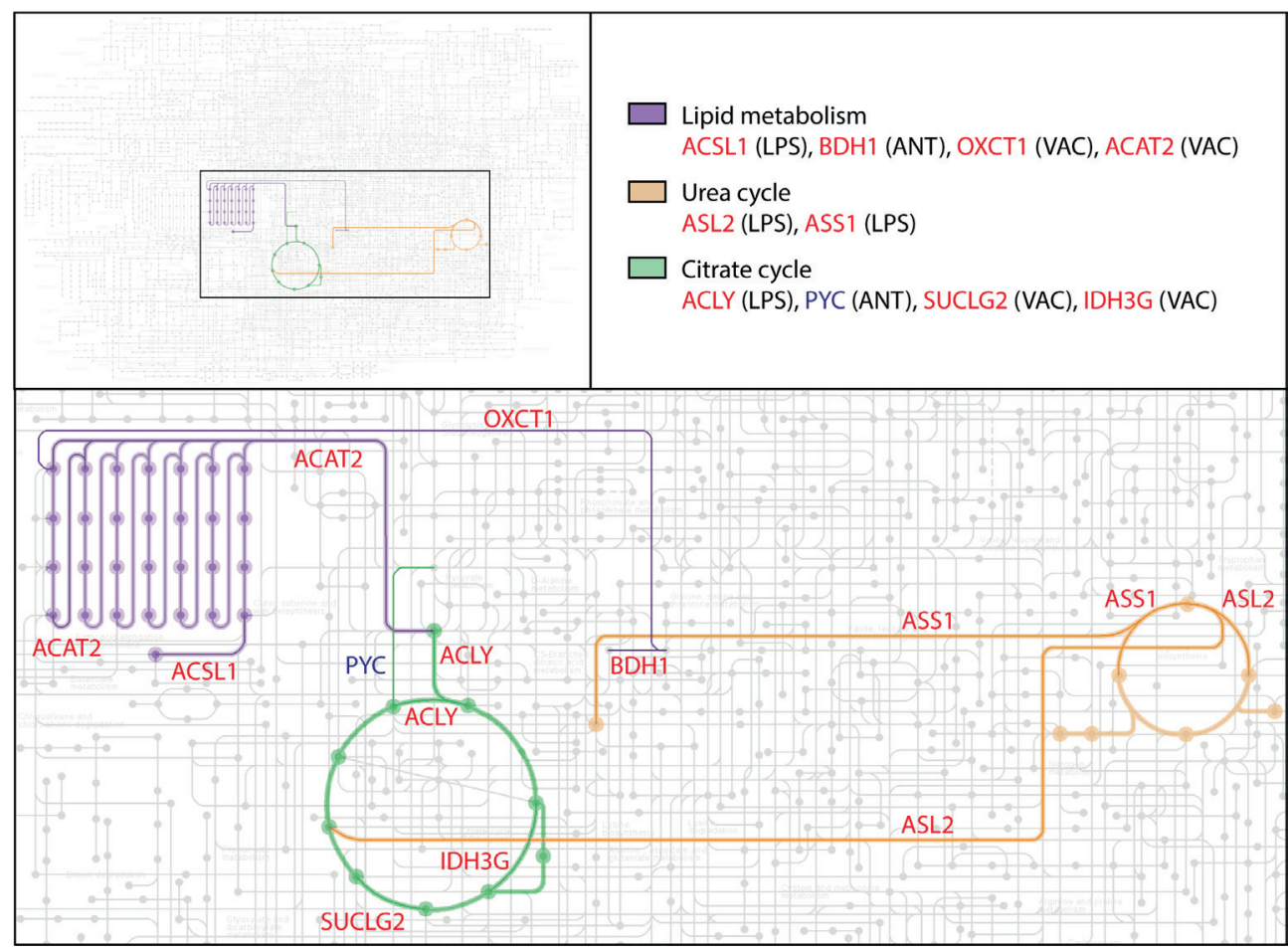

Figure 3. Interconnected metabolic pathways of chBMDCs change upon stimulation. The KEGG metabolic pathways map (gga0I 100) was modified to show differentially expressed proteins after stimulation with LPS, IBV antigen or IBV + NDV vaccine. Downregulated proteins are shown in blue and upregulated proteins are shown in red. The differentially expressed proteins could be categorized in members of lipid metabolism (purple), the urea cycle (orange) or citrate cycle (green).

were mapped to KEGG proteasome pathway (Figure 4), which demonstrated that two upregulated DEPs of chBMDCs stimulated with IBV + NDV vaccine, PSMA5 $(\alpha 5)$ and PSMBI ( $\beta 6)$, are part of the proteasomal $20 \mathrm{~S}$ core particle. The third DEP, PSMD7 (Rpn8), is part of the Rpn8-Rpn I I heterodimer with a function in removal of polyubiquitin tags before protein degradation in the proteasome [29]. A proteasomal subunit in close proximity to the Rpn8Rpn II heterodimer is PSMDI3 (Rpn9), which showed a trend towards upregulation (fold change $=4.2 ; p=0.07)$. Finally, the polyubiquitin-capturing protein PSMD4 $(\mathrm{Rpn} / 0)$ also showed a trend towards downregulation (fold change $=1.6 ; \mathrm{p}=0.07$ ).

Besides the proteins of cluster c, more proteins that were differentially expressed by chBMDCs after stimulation with IBV + NDV vaccine were found to be part of the Reactome "immune system" pathways (GGA-168256). This includes the proteasomal proteins of cluster $\mathbf{f}$, but also BLMH, FYB, RABI4, TCEBI, SCAMPI and MIF. Although the "immune system" annotation was not assigned to IFITM3, this protein has a known role in antiviral immune responses [30]. 


\section{The small number of differentially expressed proteins of antigen- stimulated chBMDCs showed limited clustering}

A limited number of DEPs was found for IBV antigen-stimulated chBMDCs. The only cluster with a majority of DEPs after stimulation with IBV antigen was cluster $\mathbf{j}$ (Figure $\mathbf{5}$ ). This cluster was annotated for Src homology 2 (SMART: SM00252; FDR $=1.5 \times 10^{-3}$ ) and 3 (SMART: SM00326; FDR $=3.2 \times 10^{-3}$ ) domains. The DEPs RIN3, MATK and SYK possess a Src homology 2 domain. Apart from this, IBV antigen seemed to have little impact on functional pathways of chBMDCs.

\section{Differentially expressed proteins of LPS-stimulated chBMDCs cluster in functional networks related to immune responses, metabolism, endocytosis, the proteasome and lysosomal enzymes}

The DEPs of LPS-stimulated chBMDCs were found overrepresented in clusters c $(8 / 14)$, d (4/8), e (4/7), f (4/7), g (6/6), h (3/6) and i (3/4) (Figure 6). Similar to stimulation with IBV + NDV vaccine, cluster c contained many DEPs after stimulation with LPS. Within this cluster, two DEPs (ELMOI and PDXK) were shared between IBV + NDV vaccine- and LPSstimulated chBMDCs. Furthermore, "regulation of actin cytoskeleton" (KEGG: gga048I0; $\mathrm{FDR}=3.3 \times 10^{-6}$ ) and "focal adhesion" (KEGG: gga045I0; FDR $\left.=3.3 \times 10^{-6}\right)$ were found as important functional enrichments. All proteins of cluster d were part of "metabolic pathways" (KEGG: gga0 I I00; FDR $=4.6 \times 10^{-7}$ ), more specifically they were part of the citrate cycle, urea cycle or fatty acid metabolism (Figure 3). Cluster e was functionally enriched for proteins containing "Src homology 3 domains" (PFAM: PFI4604; FDR = 2.2x10-6), "clathrin-mediated endocytosis" (Reactome: GGA-8856828; FDR = 1.6x 10-6) and "membrane trafficking"

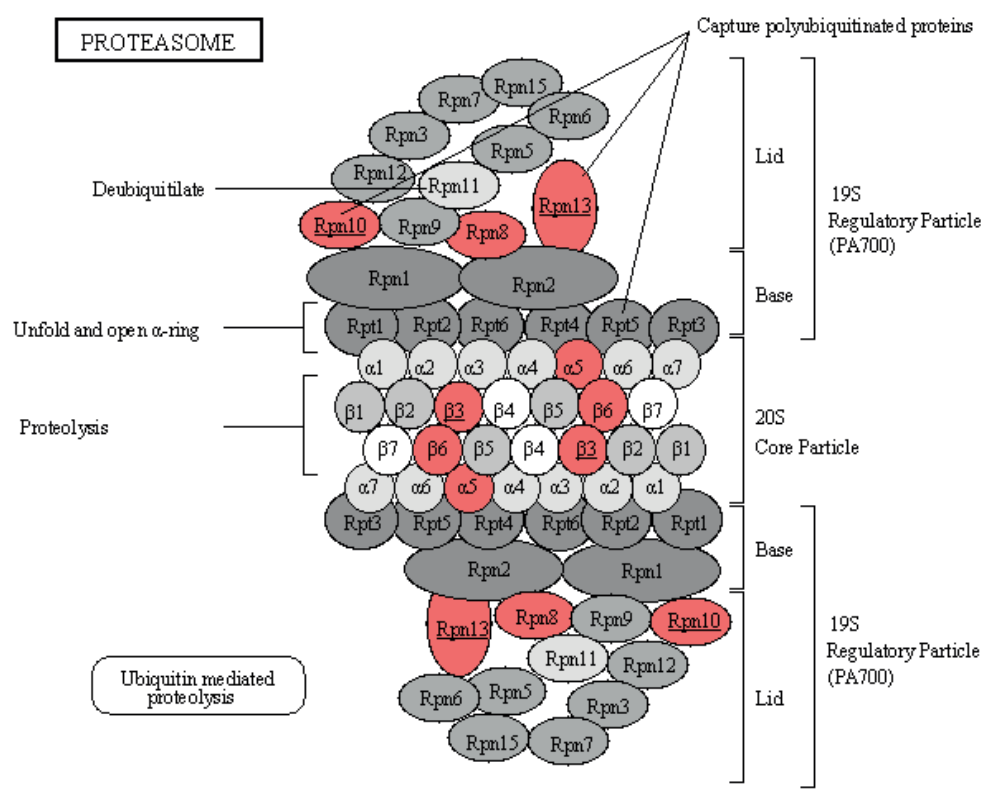

Figure 4. Differential expression of several proteasomal subunits in chBMDCs upon stimulation with LPS or IBV + NDV vaccine. The KEGG proteasome map (gga03050) shows upregulated proteins in red and others in grayscale. Underlined subunits PSMB3 ( $\beta 3$ ), PSMD4 (Rpn I0) and ADRMI (Rpn I3) were upregulated after LPS stimulation, whereas the non-underlined subunits PSMA5 $(\alpha 5)$, PSMBI $(\beta 6)$ and PSMD7 (Rpn8) were upregulated after stimulation with IBV + NDV vaccine. 
(a)

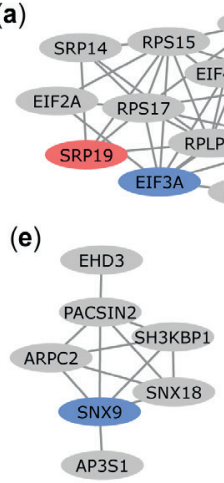

NME2

NME2

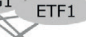

(b)

(c)

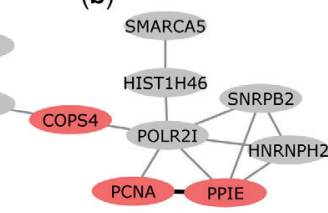

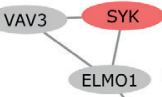

(d)

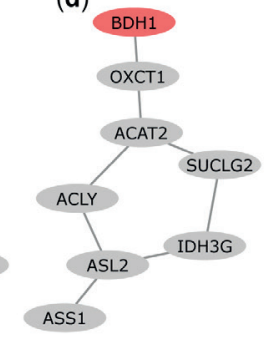

(h)

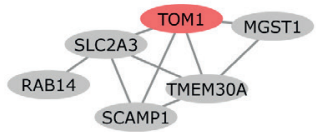

(i)

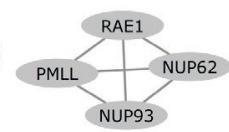

(j)

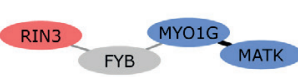

(k)

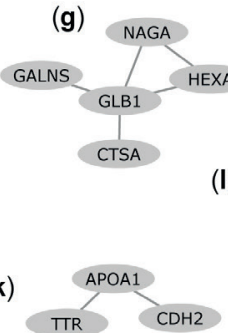

(I)

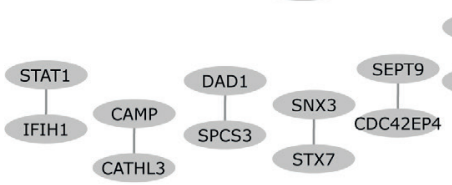

PRDX1

CAT

Figure 5. Cytoscape STRING network of differentially expressed proteins shows clusters of differentially expressed proteins after IBV antigen stimulation. Upregulated differentially expressed proteins are shown in red and downregulated differentially expressed proteins are shown in blue. Proteins in grey were not significantly differentially expressed after IBV antigen stimulation. Networks are only shown for proteins with a minimum required interaction score of 0.700 (high confidence). STRING interactions between proteins that are both differentially expressed by antigen exposure are shown in black, otherwise in grey. The letters indicate different clusters with cluster I representing six clusters comprising two proteins each.

(Reactome: GGA- I9999I; FDR $\left.=4.6 \times 10^{-6}\right)$. Similar to stimulation with IBV + NDV vaccine, cluster $\mathbf{f}$ contained DEPs after stimulation with LPS, including the non-ATPase regulatory proteasomal subunits PSMD4 (Rpnl0) and ADRMI (Rpnl3) with a function in capturing polyubiquitinated proteins for proteolysis (Figure 4). A third proteasomal subunit, PSMC3 (Rpt5), with a function in the capture of polyubiquitinated proteins showed a trend of being upregulated after LPS stimulation (fold change $=1.3 ; p=0.053$ ). The peptide transporter TAP2, which is responsible for the translocation of proteasome-processed peptides into the endoplasmatic reticulum for loading on MHC molecules, was found upregulated after LPS stimulation (Supplementary Table S2). Cluster g comprised lysosomal enzymes (KEGG: gga04I42; FDR $=8.4 \times 10^{-13}$ ) and was completely downregulated after LPS stimulation. In addition to the proteins of cluster $\mathbf{g}$, other lysosomal enzymes were downregulated upon LPS stimulation (Supplementary Figure SI), including GAAGSD, DNasell, ACP5, and SMPDI. Furthermore, the minor lysosomal membrane protein NPC was downregulated. Cluster $\mathbf{h}$ was functionally enriched for "organic substance transport" (GO: 007I 702; FDR $=1.7 \times 10^{-5}$ ) and "neutrophil degranulation" (Reactome: GGA-6798695; FDR $=4.8 \times 10^{-5}$ ). Cluster $\mathbf{i}$ comprised four proteins that were found to be related to small ubiquitin-related modifier (SUMO) E3 ligases (Reactome: GGA-3108232; FDR = I. $1 \times 10^{-8}$ ). 
(a)

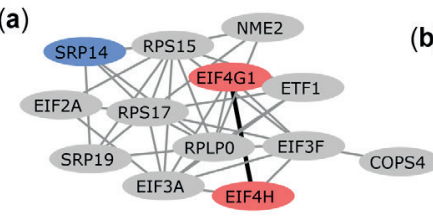

(e)

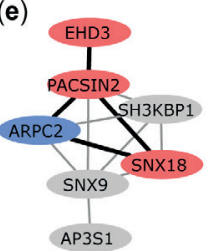

(i)

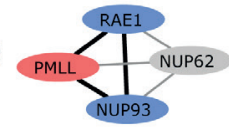

(j)

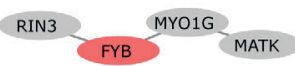

(b) (c)
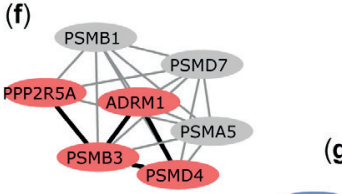

(g)

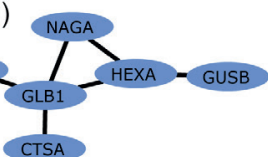

(h)

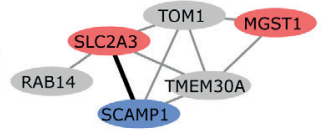

(I)
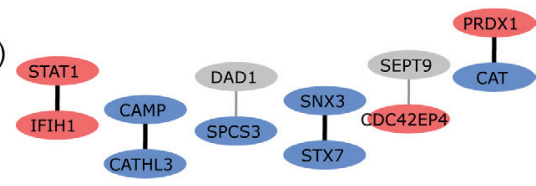

Figure 6. Cytoscape STRING network of differentially expressed proteins shows clusters of differentially expressed proteins after LPS stimulation. Upregulated differentially expressed proteins are shown in red and downregulated differentially expressed proteins are shown in blue. Proteins in grey were not significantly differentially expressed after LPS stimulation. Networks are only shown for proteins with a minimum required interaction score of 0.700 (high confidence). STRING interactions between proteins that are both differentially expressed by LPS exposure are shown in black, otherwise in grey. The letters indicate different clusters with cluster I representing six clusters comprising two proteins each.

\section{Gene expression analysis shows partial overlap with mass spectrometry data, resulting in candidate vaccine-associated biomarkers PSMBI and PLIN2}

Finally, we set out to investigate whether any of the DEPs could also be detected as differentially expressed genes (DEGs) by RT-qPCR, a method that could directly be used to evaluate biomarker expression in chickens immune cells. Similar to the proteomics data, chBMDCs were stimulated for $24 \mathrm{~h}$ with IBV + NDV vaccine, IBV antigen or LPS. The eight most significantly upregulated DEPs of IBV + NDV vaccine-stimulated chBMDCs were selected to evaluate gene expression levels (Figure 7a). Moreover, three DEPs were selected for IBV antigen-stimulated chBMDCs (Figure 7b) and four DEPs for LPSstimulated chBMDCs (Figure 7c). Analysis of gene expression showed that the three out of eight tested vaccine-related DEPs were also found upregulated on mRNA, including PSMBI $(p=0.042)$ and PLIN2 $(p=0.002)$, which were significantly upregulated, (Figure 7d) and SEPT9, which tended to be upregulated $(p=0.055)$. None of the selected DEPs of antigenstimulated chBMDCs were differentially expressed on mRNA level (Figure 7e). Two out of four DEPs of LPS-stimulated chBMDCs, GBP4L and ACSLI, were found as upregulated DEGs (Figure 7f). Analyzing mRNA expression levels of all DEP after $8 \mathrm{~h}$ rather than $24 \mathrm{~h}$ stimulation showed an upregulation of PLIN2 (I.96-fold) of vaccine-stimulated chBMDCs, an upregulation of GBP4L (8.5-fold) and ACSLI (9.8-fold) of LPS-stimulated chBMDCs, and a downregulation of EPB4IL2 (3.0-fold) of LPS-stimulated chBMDCs, while other genes were unaffected (data not shown). 

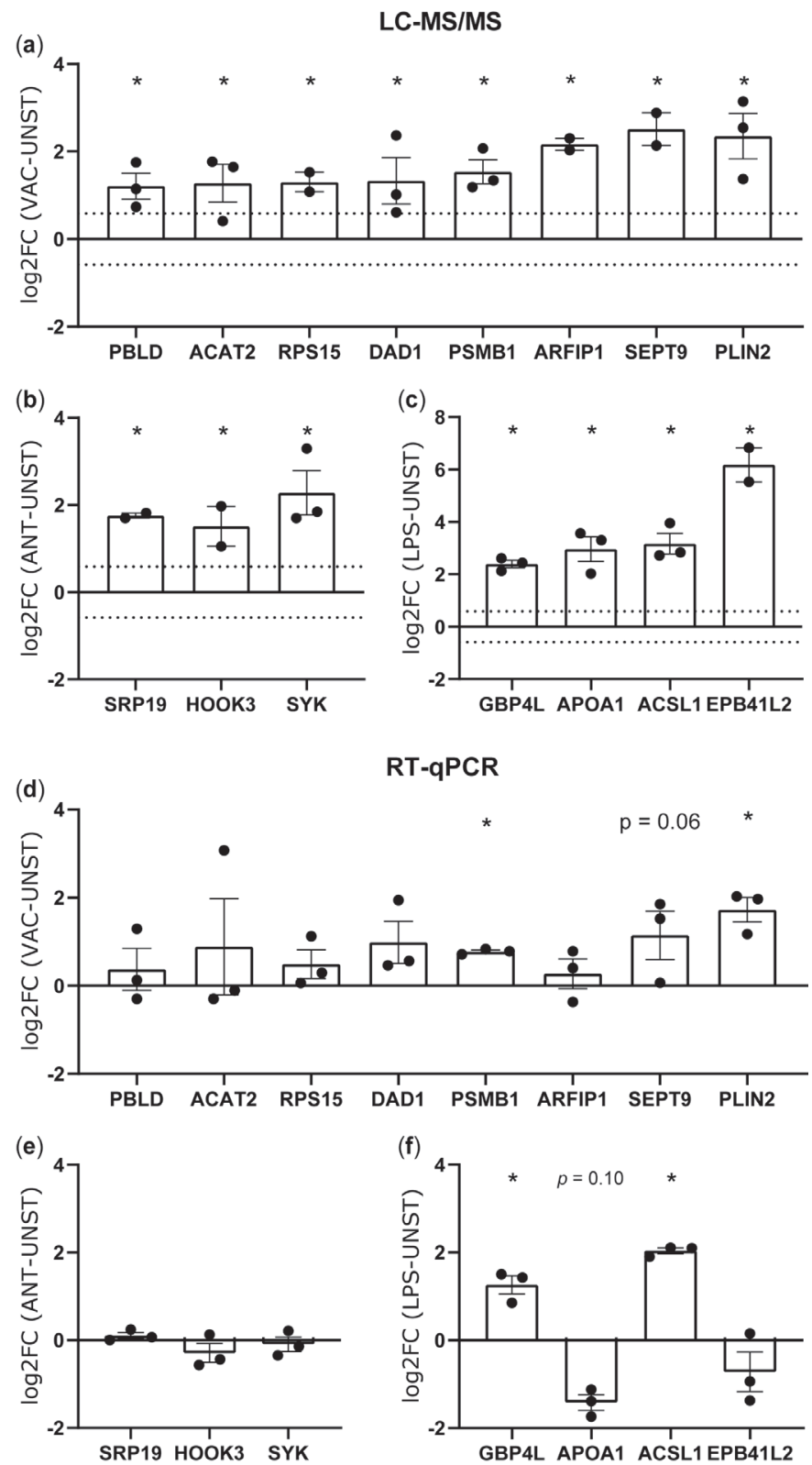

Figure 7. Comparison of protein and gene expression levels of differentially expressed proteins found to be most upregulated in mass spectrometry. Differentially expressed proteins of (a) IBV + NDV vaccine-, (b) IBV antigen- (c) and LPS-stimulated chBMDCs were selected for gene expression analysis using qPCR (resp. d-f). The expression of proteins and genes was analyzed for three independent replicates. The error bars show the SEM, and the dotted lines represent the cutoff-values of I.5-fold change that was used to select differentially expressed proteins in mass spectrometry. Statistically significant differences between stimulated and unstimulated chBMDCs are indicated by $*$ for $p<0.05$. 


\section{Discussion}

To our best knowledge, this study was the first attempt to discover biomarkers for inactivated poultry vaccines by investigating vaccine-induced immune responses with proteomics. To do so, we stimulated chBMDCs with an inactivated IBV + NDV poultry vaccine containing a mineral oil adjuvant or inactivated IBV antigen only and we analyzed the cell lysates by LC$M S / M S$, which resulted in a list of DEPs that were also evaluated for expression on mRNA level using RT-qPCR. Furthermore, chBMDCs were stimulated with E. coli LPS as a strong stimulator of innate immune cells, as demonstrated in previous studies [14-19,31,32], and was used to validate our methodology and to generate new insights in cellular processes involved in LPS driven maturation of chBMDCs.

Mass spectrometry analysis led to the identification of 118 DEPs in chBMDCs after stimulation with LPS, 66 DEPs after stimulation with IBV + NDV vaccine and 25 DEPs after stimulation with IBV antigen. For each stimulus, $20-25 \%$ of the DEPs were found to be uniquely expressed in either the unstimulated group or any of the stimulated groups, whereas the protein expression levels were absent or below the detection limit of the mass spectrometer in the other groups. Although statistics could not be performed for these proteins, the qualitative differences were still considered to be of practical significance. In the STRING analysis, DEPs based on qualitative differences made up $23 \%$ and were therefore not over- or underrepresented compared to DEPS based on quantitative differences. Moreover, DEPs based on qualitative differences were randomly distributed among the clusters of the STRING network (Supplementary Table S5). Therefore, inclusion of DEPs based on qualitative differences strengthened our STRING enrichment analysis without significantly changing the clusters observed.

Stimulation of chBMDCs with E. coli LPS resulted in most DEPs, which were associated with the (innate) immune system, regulation of the cytoskeleton, metabolic pathways, endocytosis, the lysosome, the proteasome, and SUMO E3 ligases. A previous study reported in vivo effects of LPS at the transcriptomic level [33], showing increased transcription of genes associated with inflammatory responses, regulation of the cytoskeleton and cell migration in the bursa of Fabricius, a lymphoid organ only found in birds. This corresponds with the DEPs of the current study, annotated for functions in the innate immune system and regulation of the cytoskeleton. Moreover, many DEPs of LPS-stimulated chBMDCs, including proteins with functions in the innate immune system, have previously been described to be significantly increased at the gene expression level in the chicken caecum and spleen upon bacterial infection with S. enteritidis [34,35]. Finally, the SUMO E3 ligase PMLL was upregulated by LPS stimulation, which is in agreement with a study about the mammalian homologue PML that showed its importance for immune cell activation by LPS [36].

The results of this study shed light on mechanistic aspects of LPS stimulation that result in chBMDC maturation [14-19,31,32]. First of all, increased expression of STATI was observed, in agreement with its requirement for DC maturation [37]. Secondly, we observed a strong downregulation of lysosomal hydrolases after LPS stimulation, in line with previous reports showing a decreased uptake of antigens by endocytosis or phagocytosis after LPS stimulation [14]. This effect is in accordance with studies in human and murine BMDCs showing reduced recruitment of lysosomal hydrolases to phagosomes, reduced phagosomal acidification and 
reduced phagolysosomal activity that together preserve exogenous antigens for (cross-) presentation by mature DCs [38-4I]. Thirdly, we have observed an upregulation of the non-ATPase regulatory subunits PSMD4 and ADRMI of the proteasome. The increased expression of regulatory proteasomal subunits after LPS stimulation has been reported to facilitate more efficient antigen processing in murine BMDCs [4I]. The downregulation of lysosomal activity, the upregulation of proteasomal activity and the upregulation of TAP2, which is required for the transport of proteasome-processed peptides to the endoplasmic reticulum for loading on MHC molecules, suggest that antigen-presentation by chBMDCs increased as a consequence of DC maturation after LPS stimulation, similar to murine BMDCs [39,4I].

Several DEPs of LPS-stimulated chBMDCs are involved in antimicrobial activity, even though these proteins did not cluster into one STRING network. Four of these proteins were upregulated metabolic enzymes involved in the production of inflammatory mediators, namely ASL2, ASSI, IRGI and ACSLI. The DEPs ASL2 and ASSI are involved in nitric oxide production [42], ACSLI, has a role in the synthesis of the inflammatory mediator prostaglandin E2 [43], and IRGI produces itaconate [44], all of which are inflammatory mediators with antimicrobial effects. IRGI [4I] and ACSL [45] were previously shown to be upregulated in murine BMDCs stimulated with LPS or bacteria. Furthermore, the upregulated DEPs AVD and GBP4L have direct antimicrobial effects [44]. GBP4L was also found as DEG by RT-qPCR and is involved in the activation of innate immune cells by LPS and bacteria [46]. In contrast, the antimicrobial cathelicidins CATHL2 and CATHL3, known to neutralize LPS $[47,48]$, were found downregulated in the chBMDCs. Taken together, our proteomics data showed that LPS stimulation induced changes associated with cellular processes including DC maturation and antimicrobial activity that have not been described before at the protein level for chicken immune cells. In addition to its function as a strong immunostimulatory control, thereby verifying our methodology, LPS stimulation led to increased insight in relevant changes in protein expression in chBMDCs.

For IBV antigen-stimulated chBMDCs, a limited number of DEPs was observed. Of these, SYK was the strongest upregulated DEP. SYK was previously shown to be upregulated in response to uric acid crystals in humans [49]. Uric acid crystals are also present in the IBV antigens, since these are dissolved in allantoic fluid from embryonated chicken eggs [50]. Previously, we have shown that allantoic fluid has immunostimulatory properties, potentially through the presence of uric acid crystals, leading to increased Fc receptor-mediated phagocytosis in macrophages [5I], in which SYK is likely to be involved [52].

The STRING analysis for vaccine-stimulated chBMDCs showed that the DEPs identified were largely related to immune response, the proteasome, metabolism, and mRNA translation. Since there was no overlap between chBMDCs stimulated with the inactivated IBV antigen and those that were stimulated with vaccine, the effects of the vaccine are likely to be induced by either the mineral oil adjuvant or inactivated NDV antigen as the other components of the bivalent vaccine. Due to its slow metabolization, mineral oil acts as a depot that releases antigen over a long period of time, during which strong humoral immune responses can develop [53-57]. Some upregulated DEPs of vaccine-stimulated chBMDCs involved in the storage (PLIN2 and TMEM23A) and metabolism (LIPA, OCXTI and ACATI) of lipids, or part of the citric acid cycle (SUCLG2 and IDH3G), may together contribute to 
the metabolism of internalized mineral oil adjuvant and hence to the release of antigen in course of time. Two more upregulated proteins involved in lipid storage, SYAPI and RABI8, were very close to significance $(p<0.06)$. In contrast, OSBPIIL and APOAI involved in lipid transport were found as downregulated DEPs. The storage of lipids in IBV + NDVstimulated chBMDCs is in agreement with microscopic observations (Supplementary Figure S2d). Lipid droplets have been described as important for the induction of crosspresentation by saponin-based adjuvants [58]. PLIN2 was also found as a DEG and might therefore be particularly useful as a biomarker of the mineral oil-adjuvanted vaccine since its expression can be evaluated with RT-qPCR in addition to mass spectrometry.

Studies in mice suggest that water-in-oil-adjuvanted vaccines induce the release of damageassociated molecular patterns (DAMPs) by stressed or damaged cells, which promotes the differentiation of follicular helper T cells [59] and the induction of humoral adaptive immune responses [60]. In our present study, we found significantly DEPs in chBMDCs stimulated with the IBV + NDV vaccine involved in the innate immune response. The DEPs VAV3 and ELMOI, both involved in the formation of phagocytic cups, were downregulated, which fits to the functional change of DCs, from uptake of antigens to presentation of antigens, during DC maturation. Moreover, the proteasomal subunits PSMA5, PSMBI and PSMD7 play a role in antigen processing and were found significantly upregulated. Of these, PSMBI was also found as an upregulated DEG. In addition, the upregulated DEP BLMH has been implicated in antigen-processing for presentation on $\mathrm{MHC}-\mathrm{I}$ molecules, downstream of the proteasome [6I]. Finally, the pro-inflammatory cytokine MIF, which acts as a DAMP [62], was found to be significantly upregulated in response to the vaccine.

The aim of this study was to identify protein targets to be used for in vitro quality assessment of inactivated poultry vaccines and to generate new insights in aspects of the mechanismsof-action by which these vaccines as well as LPS activate chicken DCs. Stimulation with LPS led to decrease expression of DEPs associated with phagolysosomal activity, thereby preserving exogenous antigens for (cross-)presentation, and to increased expression of DEPs associated with proteasomal antigen processing, which together suggests that antigen presentation increases during chBMDC maturation. Furthermore, LPS stimulation led to an increase in DEPs associated with antimicrobial activity. For IBV antigen- and IBV + NDV vaccine-stimulated chBMDCs, no overlap was observed between the DEPs, which suggests that the DEPs of IBV + NDV vaccine-stimulated chBMDCs were induced by the mineral oil adjuvant, NDV antigen or the combination of both. The DEPs of vaccine-stimulated chBMDCs were found largely associated with mRNA translation, immune responses, lipid metabolism and proteasomal degradation, which provides new insights about the effects that inactivated poultry vaccines have on chicken DCs. Furthermore, two significantly upregulated DEPs of vaccine-stimulated chBMDCs included PLIN2, involved in the storage of lipid droplets, and PSMBI, a proteasomal subunit, and were also found as upregulated DEGs using RTqPCR. Future studies should include different conforming as well as non-conforming vaccine batches to assess the ability of the identified DEPs and DEGs to discriminate between batches of good and poor quality. The identification of these vaccine-associated biomarkers may facilitate the transition from in vivo to cell-based quality assessment of inactivated poultry vaccines. 


\section{Acknowledgements}

The authors thank Bart L. van den Eshof for his help with creating a strategy to perform the data analysis and Alice E.J.A.M. Sijts for her critical reading of the manuscript. This project has received funding from the Innovative Medicines Initiative 2 Joint Undertaking under grant agreement No II 5924 (VAC2VAC). This Joint Undertaking receives support from the European Union's Horizon 2020 research and innovation programme and EFPIA. The contents of this article represent the authors' scientific conclusions and neither IMI nor the European Union, EFPIA, or any Associated Partners are responsible for any use that may be made of the information contained therein. 


\section{Supplementary Materials}

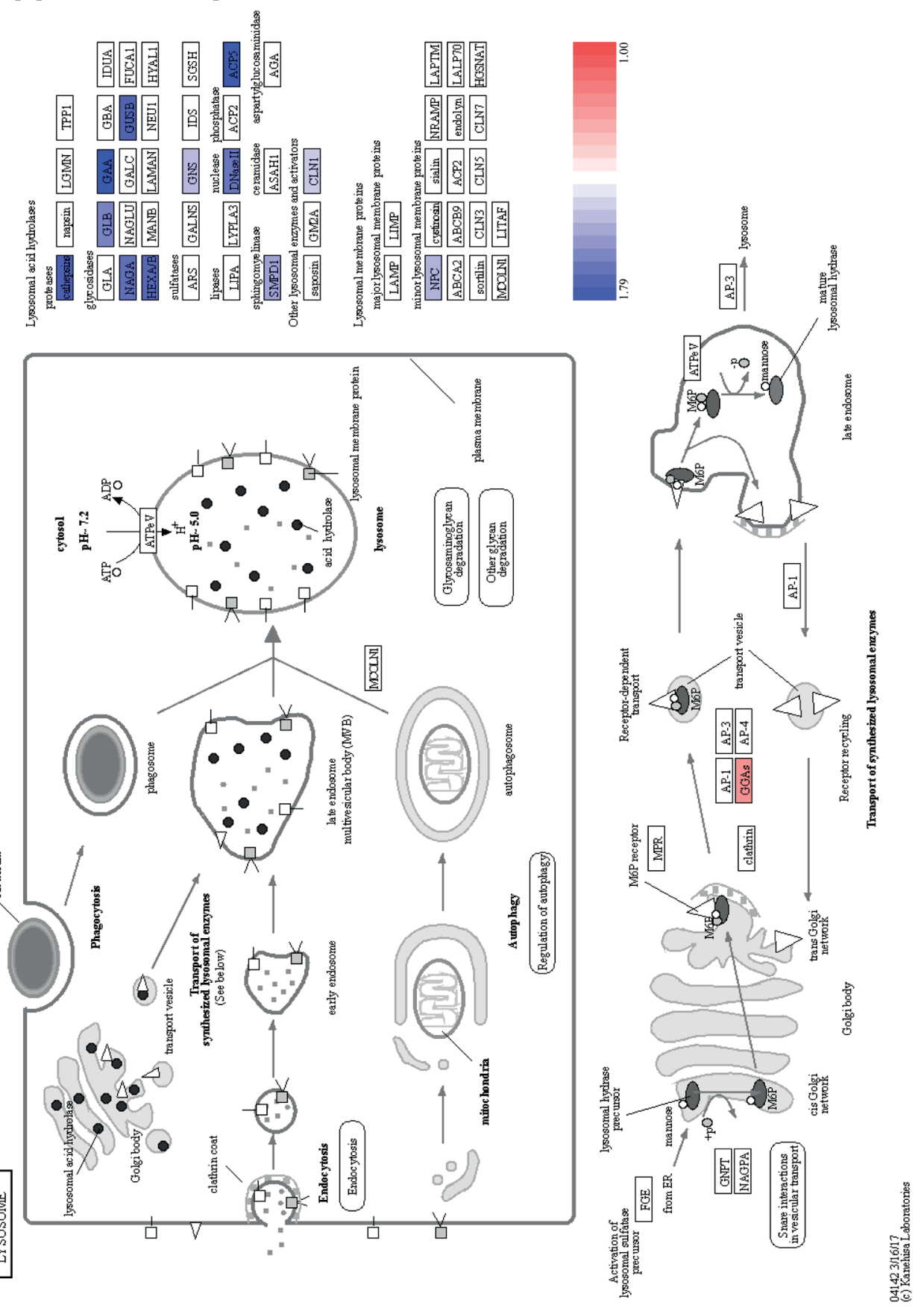

Supplementary Figure SI. Stimulation of chBMDCs with LPS led to the significant downregulation of lysosomal enzymes. The KEGG lysosome map (gga04I42) shows differentially expressed proteins of LPS-stimulated chBMDCs in the lysosome. Downregulated proteins are shown in blue and upregulated proteins are shown in red. 
(a)

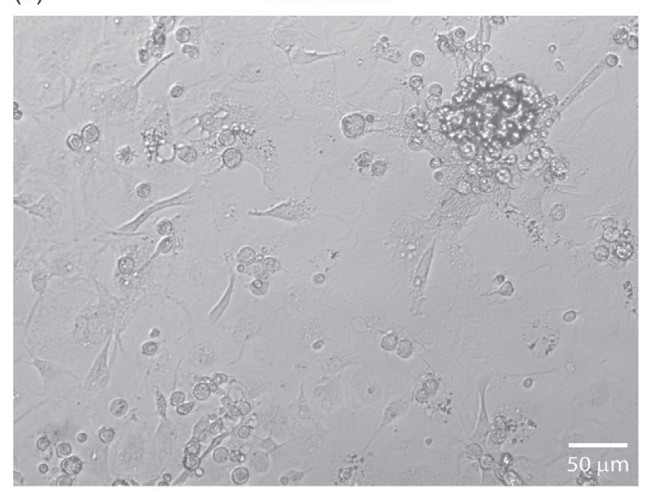

(c)

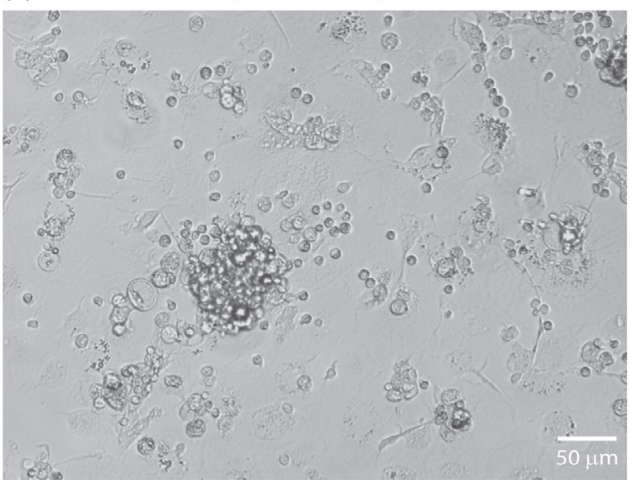

(b)

$100 \mathrm{ng} / \mathrm{ml}$ LPS

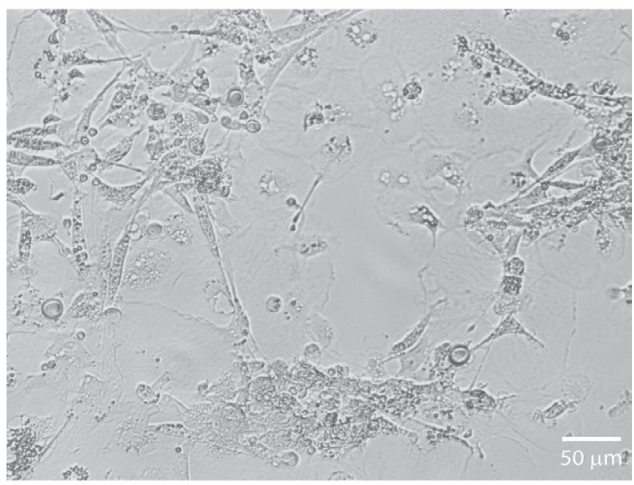

(d)

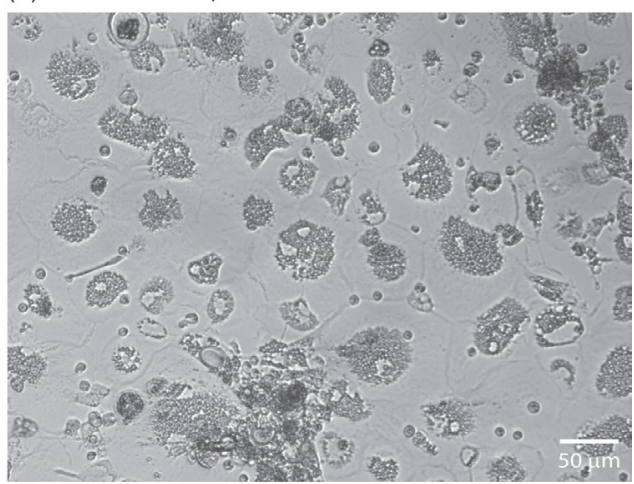

Supplementary Figure S2. The morphology of chBMDCs changes upon stimulation. The chBMDC cultures were left unstimulated (a) or were stimulated with $100 \mathrm{ng} / \mathrm{ml}$ LPS (b), $10 \mu \mathrm{l} / \mathrm{ml} \mathrm{IBV}$ antigen (c) or $30 \mu \mathrm{l} / \mathrm{ml} I B V+N D V$ vaccine (d). Subsequently, the cell morphology of the cultures was captured with brightfield light microscopy at 100x magnification.

Supplementary Tables SI-S5. These datafiles can be provided by the authors on request. 


\section{References}

I. EDQM. European Pharmacopoeia. 10.5th. Strasbourgh, France: European Department for the Quality of Medicines; 2020.

2. Cozigou G, Crozier J, Hendriksen C, Manou I, Ramirez-Hernandez T, Weissenhorn R. The European Partnership for Alternative Approaches to Animal Testing (EPAA): promoting alternative methods in Europe and beyond. J Am Assoc Lab Anim Sci (2015) 54(2):209-213.

3. Claassen I. Case study of development, validation, and acceptance of a non-animal method for assessing veterinary vaccine potency. Procedia Vaccinol (20II) 5:175-183. doi:10.1016/J. PROVAC.2011.10.017

4. Metz B, Hoonakker M, Uittenbogaard JP, Weyts M, Mommen GPM, Meiring HD, et al. Proteome analysis is a valuable tool to monitor antigen expression during upstream processing of wholecell pertussis vaccines. J Proteome Res (2017) 16(2):528-537. doi:10.1021/acs.jproteome.6b00668

5. Hoonakker ME, Verhagen LM, Pupo E, De Haan A, Metz B, Hendriksen CFM, et al. Vaccinemediated activation of human TLR4 is affected by modulation of culture conditions during wholecell pertussis vaccine preparation. PLoS One (2016) I I (8). doi:I0.137I/journal.pone.016I428

6. Hendriksen CF. Replacement, reduction and refinement alternatives to animal use in vaccine potency measurement. Expert Rev Vaccines (2009) 8(3):3 I3-322. doi: I0.1586/ I4760584.8.3.3 I3

7. Momose H, Mizukami T, Kuramitsu M, Takizawa K, Masumi A, Araki K, et al. Establishment of a new quality control and vaccine safety test for influenza vaccines and adjuvants using gene expression profiling. PLoS One (20I5) I0(4). doi:I0.137I/journal.pone.0124392

8. Sasaki E, Momose H, Hiradate Y, Ishii KJ, Mizukami T, Hamaguchi I. In vitro marker gene expression analyses in human peripheral blood mononuclear cells: A tool to assess safety of influenza vaccines in humans. J Immunotoxicol (2018) I5(I):53-62. doi:I0.1080/I54769IX.20I8.I447052

9. Querec TD, Akondy RS, Lee EK, Cao W, Nakaya HI, Teuwen D, et al. Systems biology approach predicts immunogenicity of the yellow fever vaccine in humans. Nat Immunol (2009) 10(I): I I6125. doi:10.1038/ni. 1688

10. Ahmed SS, Black S, Ulmer J. New developments and concepts related to biomarker application to vaccines. Microb Biotechnol (20I2) 5(2):233-240. doi:I0.I II I/j.I75I-79I5.20 I I.00277.x

II. Vandebriel R, Hoefnagel MMN. Dendritic cell-based in vitro assays for vaccine immunogenicity. Hum Vaccin Immunother (20I2) 8(9):I323-I325. doi:I0.4I6I/hv.2I350

12. Bande F, Arshad SS, Omar AR, Hair-Bejo M, Mahmuda A, Nair V. Global distributions and strain diversity of avian infectious bronchitis virus: A review. Anim Heal Res Rev (2017) 18(I):70-83. doi: $10.1017 / S \mid 466252317000044$

13. Bello MB, Yusoff K, Ideris A, Hair-Bejo M, Peeters BPH, Omar AR. Diagnostic and vaccination approaches for Newcastle disease virus in poultry: The current and emerging perspectives. Biomed Res Int (2018) 2018. doi:10.1 I55/2018/7278459

14. Wu Z, Rothwell L, Young JR, Kaufman J, Butter C, Kaiser P. Generation and characterization of chicken bone marrow-derived dendritic cells. Immunology (2010) I29(I):I33-145. doi:10.1 III/ j.1365-2567.2009.03129.x

15. van den Biggelaar RHGA, Arkesteijn GJA, Rutten VPMG, van Eden W, Jansen CA. In vitro chicken bone marrow-derived dendritic cells comprise subsets at different states of maturation. Front Immunol (2020) II. doi: I0.3389/fimmu.2020.00I4 I

16. de Geus ED, Jansen CA, Vervelde L. Uptake of particulate antigens in a nonmammalian lung: phenotypic and functional characterization of avian respiratory phagocytes using bacterial or viral antigens. J Immunol (20I2) I88(9):45I6-4526. doi:I0.4049/jimmunol. I 200092

17. Yasmin AR, Yeap SK, Tan SW, Hair-Bejo M, Fakurazi S, Kaiser P, et al. In vitro characterization of chicken bone marrow-derived dendritic cells following infection with very virulent infectious bursal disease virus. Avian Pathol (20I5) 44(6):452-462. doi: 10.1080/03079457.20I5.1084997

18. Liang J, Yin Y, Qin T, Yang Q. Chicken bone marrow-derived dendritic cells maturation in response to infectious bursal disease virus. Vet Immunol Immunopathol (20I5) I64:5I-55. doi:10.1016/j.vetimm.2014.12.012 
19. Van Goor A, Slawinska A, Schmidt CJ, Lamont SJ. Distinct functional responses to stressors of bone marrow derived dendritic cells from diverse inbred chicken lines. Dev Comp Immunol (2016) 63:96-II0. doi:10.1016/J.DCI.2016.05.016

20. Meiring HD, Heeft E van der, Hove GJ ten, Jong APJM de. Nanoscale LC-MS(n): technical design and applications to peptide and protein analysis. J Sep Sci (2002) 25(9):557-568. doi:10.1002/161593।4(2002060I)25:9<557::AID-JSSC557>3.0.CO;2-F

2I. Van De Waterbeemd B, Mommen GPM, Pennings JLA, Eppink MH, Wijffels RH, Van Der Pol LA, et al. Quantitative proteomics reveals distinct differences in the protein content of outer membrane vesicle vaccines. J Proteome Res (2013) I2(4): 1898-1908. doi: I0.1021/pr30 I208g

22. Kooijman S, Brummelman J, van Els CACM, Marino F, Heck AJR, van Riet E, et al. Vaccine antigens modulate the innate response of monocytes to $\mathrm{Al}(\mathrm{OH}) 3$. PLoS One (20I8) 13(5):e0197885. doi:I0.137|/journal.pone.0197885

23. Kooijman S, Brummelman J, van Els CACM, Marino F, Heck AJR, Mommen GPM, et al. Novel identified aluminum hydroxide-induced pathways prove monocyte activation and proinflammatory preparedness. J Proteomics (2018) I75:I44-I55. doi:I0.1016/j.jprot.2017.12.02I

24. Tyanova S, Temu T, Sinitcyn P, Carlson A, Hein MY, Geiger T, et al. The Perseus computational platform for comprehensive analysis of (prote)omics data. Nat Methods (20I6) 13(9):73I-740. doi:10.1038/nmeth.390I

25. Szklarczyk D, Gable AL, Lyon D, Junge A, Wyder S, Huerta-Cepas J, et al. STRING vI I: Proteinprotein association networks with increased coverage, supporting functional discovery in genome-wide experimental datasets. Nucleic Acids Res (2019) 47(DI):D607-D6I3. doi:I0.1093/ nar/gkyl|3|

26. Shannon P, Markiel A, Ozier O, Baliga NS, Wang JT, Ramage D, et al. Cytoscape: A software environment for integrated models of biomolecular interaction networks. Genome Res (2003) I3(I I):2498-2504. doi:10.1 I0I/gr.1239303

27. Hochberg Y, Benjamini Y. More powerful procedures for multiple significance testing. Stat Med (1990) 9(7):8II-8I8. doi:I0.1002/sim.47800907I0

28. Kanehisa M, Goto S, Sato Y, Furumichi M, Tanabe M. KEGG for integration and interpretation of large-scale molecular data sets. Nucleic Acids Res (20I2) 40(DI):DI09-DII4. doi:I0.1093/ nar/gkr988

29. Pathare GR, Nagy I, Śledź P, Anderson DJ, Zhou HJ, Pardon E, et al. Crystal structure of the proteasomal deubiquitylation module Rpn8-Rpn I I. Proc Natl Acad Sci U S A (20 I4) I I I (8):29842989. doi: I0.1073/pnas. I 400546III

30. Spence JS, He R, Hoffmann HH, Das T, Thinon E, Rice CM, et al. IFITM3 directly engages and shuttles incoming virus particles to lysosomes. Nat Chem Biol (2019) I5(3):259-268. doi:10.1038/ s4I589-018-02 I3-2

31. Wu Z, Hu T, Butter C, Kaiser P. Cloning and characterisation of the chicken orthologue of dendritic cell-lysosomal associated membrane protein (DC-LAMP). Dev Comp Immunol (2010) 34(2): 183-188. doi: I0.1016/J.DCl.2009.09.007

32. Wu Z, Hu T, Kaiser P. Chicken CCR6 and CCR7 are markers for immature and mature dendritic cells respectively. Dev Comp Immunol (20II) 35(5):563-567. doi:I0.10I6/J.DCI.20I0.12.0I5

33. Monson MS, Van Goor AG, Ashwell CM, Persia ME, Rothschild MF, Schmidt CJ, et al. Immunomodulatory effects of heat stress and lipopolysaccharide on the bursal transcriptome in two distinct chicken lines. BMC Genomics (2018) I9(I):643. doi: I0.1 I86/s I 2864-0 I8-5033-y

34. Matulova M, Varmuzova K, Sisak F, Havlickova H, Babak V, Stejskal K, et al. Chicken innate immune response to oral infection with Salmonella enterica serovar Enteritidis. Vet Res (2013) 44(I):37. doi: I0.1 I86/I297-97/ 6-44-37

35. Matulova M, Rajova J, Vlasatikova L, Volf J, Stepanova H, Havlickova H, et al. Characterization of chicken spleen transcriptome after infection with Salmonella enterica serovar Enteritidis. PLoS One (20I2) 7(I0). doi:I0.137I/journal.pone.0048I0I

36. Lunardi A, Gaboli M, Giorgio M, Rivi R, Bygrave A, Antoniou M, et al. A role for PML in innate immunity. Genes Cancer (20II) 2(I):I0-19. doi:I0.1I77/I94760I9II402682 
37. Jackson SH, Yu C-R, Mahdi RM, Ebong S, Egwuagu CE. Dendritic cell maturation requires STATI and is under feedback regulation by suppressors of cytokine signaling. J Immunol (2004) I72(4):2307-23I5. doi:I0.4049/jimmunol. 172.4.2307

38. Accapezzato D, Visco V, Francavilla V, Molette C, Donato T, Paroli M, et al. Chloroquine enhances human $\mathrm{CD}^{+} \mathrm{T}$ cell responses against soluble antigens in vivo. J Exp Med (2005) 202(6):8I7-828. doi:10.1084/jem.2005II06

39. Alloatti A, Kotsias F, Magalhaes JG, Amigorena S. Dendritic cell maturation and cross-presentation: timing matters! Immunol Rev (2016) 272(I):97-I08. doi:I0.1 I I I/imr. 12432

40. Lennon-Duménil AM, Bakker AH, Maehr R, Fiebiger E, Overkleeft HS, Rosemblatt M, et al. Analysis of protease activity in live antigen-presenting cells shows regulation of the phagosomal proteolytic contents during dendritic cell activation. J Exp Med (2002) 196(4):529-539. doi: 10.1084/jem.20020327

4I. Pauwels AM, Härtlova A, Peltier J, Driege Y, Baudelet G, Brodin P, et al. Spatiotemporal changes of the phagosomal proteome in dendritic cells in response to LPS stimulation. Mol Cell Proteomics (2019) 18(5):909-922. doi:10.1074/mcp.RAII9.001316

42. Rychlik I, Elsheimer-Matulova M, Kyrova K. Gene expression in the chicken caecum in response to infections with non-typhoid Salmonella. Vet Res (20I4) 45(I). doi: I0.I I86/s I3567-0 I4-0I I9-2

43. Kanter JE, Kramer F, Barnhart S, Averill MM, Vivekanandan-Giri A, Vickery T, et al. Diabetes promotes an inflammatory macrophage phenotype and atherosclerosis through acyl-CoA synthetase I. Proc Natl Acad Sci U S A (2012) I09(I2):E7 I5-E724. doi: I0. I073/pnas. I I I 600 I09

44. Price J V., Russo D, Ji DX, Chavez RA, Dipeso L, Lee AYF, et al. IRGI and inducible nitric oxide synthase act redundantly with other interferon-gamma-induced factors to restrict intracellular replication of legionella pneumophila. MBio (2019) 10(6). doi:I0.I I 28/mBio.02629- 19

45. Rubinow KB, Wall VZ, Nelson J, Mar D, Bomsztyk K, Askari B, et al. Acyl-CoA synthetase I Is induced by gram-negative bacteria and lipopolysaccharide and is required for phospholipid turnover in stimulated macrophages. J Biol Chem (2013) 288(I4):9957-9970. doi:10.1074/jbc. MII3.458372

46. Kim BH, Shenoy AR, Kumar P, Das R, Tiwari S, MacMicking JD. A family of IFN- $\gamma$-inducible 65kD GTPases protects against bacterial infection. Science (20II) 332(6030):717-72I. doi: I0. I I 26/ science. 1201711

47. Scheenstra MR, van den Belt M, Tjeerdsma-van Bokhoven JLM, Schneider VAF, Ordonez SR, van Dijk A, et al. Cathelicidins PMAP-36, LL-37 and CATH-2 are similar peptides with different modes of action. Sci Rep (2019) 9(I):I-12. doi: I0.1038/s4I598-019-4I246-6

48. Coorens M, Scheenstra MR, Veldhuizen EJA, Haagsman HP. Interspecies cathelicidin comparison reveals divergence in antimicrobial activity, TLR modulation, chemokine induction and regulation of phagocytosis. Sci Rep (2017) 7. doi:10.1038/srep40874

49. Pelka K, Latz E. Getting closer to the dirty little secret. Immunity (20II) 34(4):455-458. doi:10.1016/j.immuni.2011.04.003

50. Bolin G, Burggren WW. Metanephric kidney development in the chicken embryo: Glomerular numbers, characteristics and perfusion. Comp Biochem Physiol - A Mol Integr Physiol (2013) 166(2):343-350. doi:10.1016/j.cbpa.2013.07.01 I

5I. van den Biggelaar RHGA, van Eden W, Rutten VPMG, Jansen CA. Nitric oxide production and Fc receptor-mediated phagocytosis as functional readouts of macrophage activity upon stimulation with inactivated poultry vaccines in vitro. Vaccines (2020) 8(2):332. doi: 10.3390/vaccines8020332

52. Crowley MT, Costello PS, Fitzer-Attas CJ, Turner M, Meng F, Lowell C, et al. A critical role for Syk in signal transduction and phagocytosis mediated by Fc $\gamma$ receptors on macrophages. J Exp Med (1997) 186(7):1027-1039. doi:10.1084/jem.186.7.1027

53. Bollinger JN. Metabolic fate of mineral oil adjuvants using I4C-labeled tracers I: Mineral oil. J Pharm Sci (1970) 59(8): 1084-1088. doi:10.1002/jps.2600590804

54. Droual R, Bickford AA, Charlton BR, Kuney DR. Investigation of problems associated with intramuscular breast injection of oil-adjuvanted killed vaccines in chickens. Avian Dis (1990) 34(2):473-478. doi:10.2307//59/439 
55. Yamanaka M, Okabe T, Nakai M, Goto N. Local pathological reactions and immune response of chickens to ISA-70 and other adjuvants containing Newcastle disease virus antigen. Avian Dis (1993) 37(2):459-466. doi:10.2307/I591673

56. Herbert WJ. The mode of action of mineral-oil emulsion adjuvants on antibody production in mice. Immunology (1968) I4(3):30I-3I8.

57. Gough RE, Allan WH, Nedelciu D. Immune response to monovalent and bivalent Newcastle disease and infectious bronchitis inactivated vaccines. Avian Pathol (1977) 6(2):|3|-|42. doi: I0.1080/030794577084|822।

58. Den Brok MH, Büll C, Wassink M, De Graaf AM, Wagenaars JA, Minderman M, et al. Saponinbased adjuvants induce cross-presentation in dendritic cells by intracellular lipid body formation. Nat Commun (2016) 7(1):I-14. doi:10.1038/ncomms I3324

59. Riteau N, Radtke AJ, Shenderov K, Mittereder L, Oland SD, Hieny S, et al. Water-in-oil-only adjuvants selectively promote $\mathrm{T}$ follicular helper cell polarization through a type I IFN and IL-6dependent pathway. J Immunol (2016) 197(10):3884-3893. doi: 10.4049/jimmunol. 1600883

60. Gavin AL, Hoebe K, Duong B, Ota T, Martin C, Beutler B, et al. Adjutant-enhanced antibody responses in the absence of toll-like receptor signaling. Science (2006) 314(5807): 1936-1938. doi: I0.1 I26/science. II 35299

61. Stoltze L, Schirle M, Schwarz G, Schröter C, Thompson MW, Hersh LB, et al. Two new proteases in the MHC class I processing pathway. Nat Immunol (2000) I (5):4I3-4 I8. doi: I0.1038/80852

62. Kapurniotu A, Gokce O, Bernhagen J. The multitasking potential of alarmins and atypical chemokines. Front Med (2019) 6:3. doi:10.3389/fmed.2019.00003 


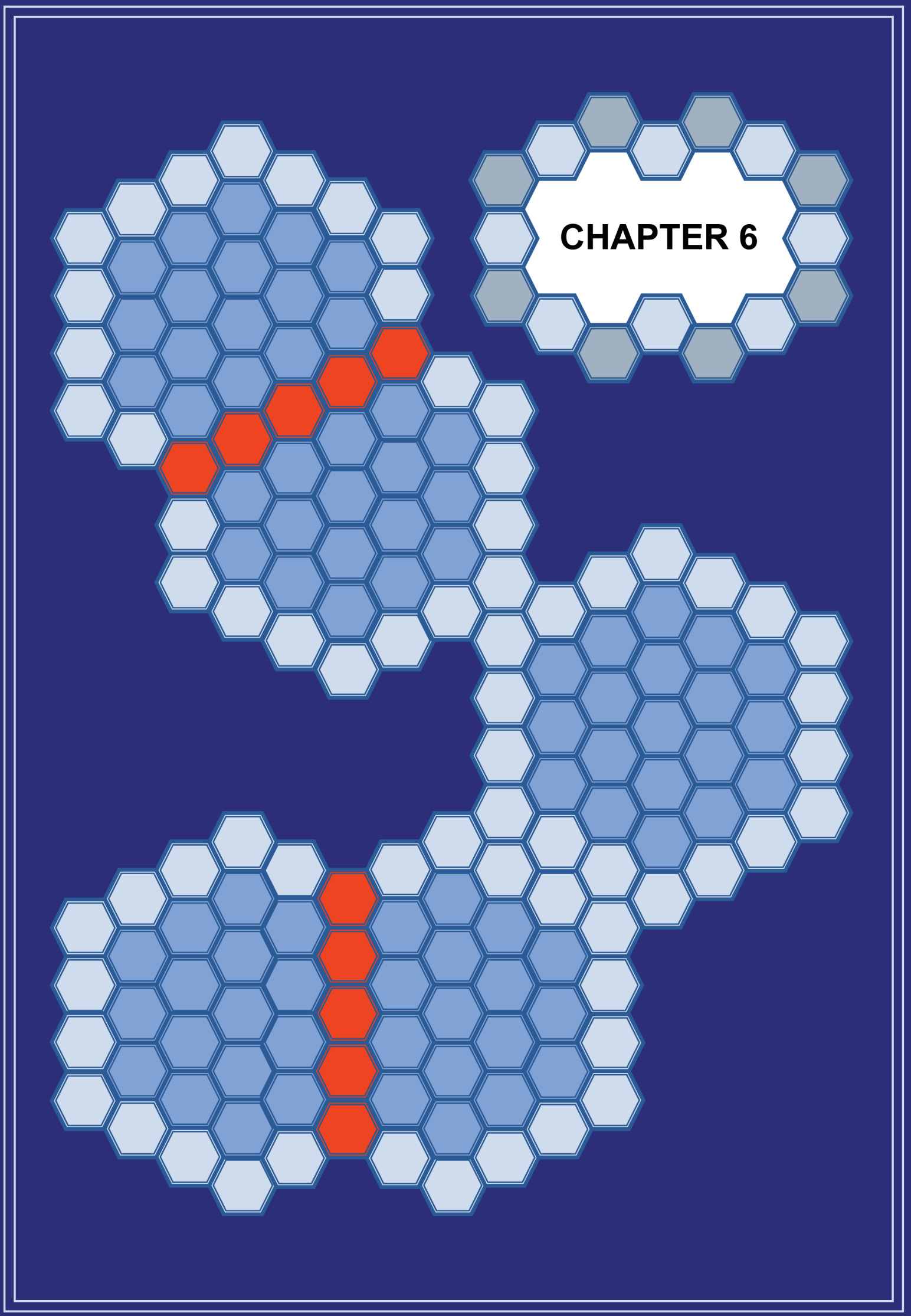




\section{Assessment of antigen-specific $T$ cell responses in chickens after vaccination with an inactivated vaccine for infectious bronchitis virus and Newcastle disease virus}

Robin H.G.A. van den Biggelaar', Daphne A. van Haarlem', Andreas Papanikolaou², Mieke G. Matthijs' ${ }^{2}$, Willem van Eden', Victor P.M.G. Rutten ${ }^{1,3}$ and Christine A. Jansen ${ }^{1,4}$

' Division of Infectious Diseases and Immunology, Department of Biomolecular Health Sciences, Faculty of Veterinary Medicine, Utrecht University, Utrecht, the Netherlands

${ }^{2}$ Division of Farm Animal Health, Department of Population Health Sciences, Faculty of Veterinary Medicine, Utrecht University, the Netherlands

${ }^{3}$ Department of Veterinary Tropical Diseases, Faculty of Veterinary Science, University of Pretoria, Pretoria, South Africa

${ }^{4}$ Cell Biology and Immunology Group, Department of Animal Sciences, Wageningen University and Research, Wageningen, the Netherlands 


\section{Abstract}

Early in life, chickens are vaccinated for infectious bronchitis virus (IBV) and Newcastle disease virus (NDV) using live attenuated vaccines. Before the laying period, layers as well as broiler breeders receive additional inactivated booster vaccines for IBV and NDV to enhance protective immunity against these diseases during the laying period. Batch quality control of inactivated poultry vaccines is often assessed in serological assays. The role of cell-mediated immunity (CMI) in the protection induced by these vaccines is less well understood. In this study, we investigated the suitability of a $\mathrm{T}$ cell proliferation assay in routine vaccine quality control of inactivated IBV/NDV vaccines. Blood was collected from laying hens before and three weeks after vaccination with an inactivated IBV/NDV vaccine. Peripheral blood mononuclear cells were isolated and stimulated ex vivo with inactivated IBV and NDV antigens, and a bivalent IBV and NDV vaccine, after which T cell proliferation was assessed. Proliferation of $\mathrm{T}$ cells was readily observed after polyclonal stimulation. Furthermore, proliferation of $T$ cells was observed upon stimulation with inactivated IBV antigens before but not after vaccination. Thus, although $T$ cell proliferation was readily observed upon polyclonal stimulation, IBV-specific $\mathrm{T}$ cell proliferative responses were limited and not affected by the additional booster vaccination. Finally, proliferation was not observed upon stimulation with inactivated NDV antigens or the bivalent vaccine, neither before nor after vaccination. Based on the present findings, we have concluded that the $T$ cell proliferation assay was not sensitive enough for routine quality control of the inactivated IBV/NDV vaccine. Whether the absence of $T$ cell proliferation in response to IBV or NDV after additional vaccination with an inactivated vaccine has a technical or biological explanation needs to be further elucidated by future studies. 


\section{Introduction}

Vaccines are important to maintain poultry health and welfare, and to prevent economic losses in the poultry industry due to infectious diseases. Chickens are vaccinated against a plethora of infectious diseases including infectious bronchitis virus (IBV) and Newcastle disease virus (NDV) before or soon after hatch using live attenuated vaccines, or alternatives like recombinant viral vector-based vaccines or DNA vaccines [I,2]. Later in life, laying hens and broiler breeders may receive additional inactivated booster vaccines for IBV and NDV to enhance protective immunity against these viruses during the laying period.

Quality of batches of inactivated poultry vaccines is evaluated routinely by assessing their performance in in vivo vaccination-challenge studies or in serological tests that evaluate the induction of antigen-specific antibodies, which is an important aspect of vaccine-mediated protection against IBV and NDV. Antibodies also protect the offspring of broiler breeders from infectious diseases during early life [3]. Whole inactivated vaccines against IBV and NDV are adjuvanted with mineral oil formulated as water-in-oil emulsions to further enhance the induction of protective antibodies [4,5]. The induction of IBV- [6] and NDV-specific T cells [7] has also been demonstrated to be important for protective immunity in infection studies.

Evaluation of antigen-specific $\mathrm{T}$ cell proliferation ex vivo could potentially be used to demonstrate $\mathrm{CMI}$ in routine vaccine quality control and complement serological assays. However, whether inactivated poultry vaccines have the capacity to stimulate antigenspecific $T$ cells remains unclear. Some studies were able to demonstrate the induction of CMI using $\mathrm{T}$ cell proliferation assays in chickens that received live attenuated vaccines against NDV [8,9] or a combination of live attenuated and inactivated vaccines against NDV $[8,10]$, whereas another study could not [II]. With regard to IBV vaccines, one study demonstrated $\mathrm{CMI}$ in chickens that received a single dose of inactivated vaccine by a small proliferative response of $T$ cells upon stimulation with IBV ex vivo [12], but the results have not been repeated since. As a consequence, knowledge about the extent to which inactivated poultry vaccines induce $\mathrm{CMI}$ is still limited.

In a previous study, a T cell proliferation assay was used to demonstrate CMI upon infection with Salmonella enteritidis in broiler chickens (Meijerink, submitted). Using the same methods, we investigated whether this $\mathrm{T}$ cell proliferation assay is suitable in routine vaccine quality control of inactivated vaccines against IBV and NDV.

\section{Materials and Methods}

\section{Animals and experimental design}

Ten Lohmann Brown Classic laying hens of 33 weeks old were housed at the animal facilities of the department of Farm Animal Health, Faculty of Veterinary Medicine, Utrecht University (Dutch Central Authority for Scientific Procedures on Animals registration number AVDI08002016642-I). The chickens were daily monitored by animal caretakers for signs of diseases, which were absent. All chickens in this study had been subjected to the standard vaccination regimen for laying hens before the onset of this study. In addition, the chickens received the inactivated booster vaccine Nobilis IB Multi (strains M4I and D274) 
+ ND by intramuscular injection (musculus pectoralis superficialis). The complete vaccination scheme is given in Table $\mathbf{I}$.

\section{Peripheral blood mononuclear cell isolation}

For nine out of ten chickens, $3 \mathrm{ml}$ heparinized blood was taken from the wing (vena ulnaris) and collected in $50 \mathrm{ml}$ Falcon tubes containing $200 \mu \mathrm{l}$ heparin (5000 IE/ml; LEO Pharma A/S, Copenhagen, Denmark) both before and 21 days after vaccination, and kept at room temperature. For one chicken, only approximately I ml blood was obtained. The heparinized blood, was diluted I:I in PBS (Lonza, Basel, Switzerland) and the peripheral blood mononuclear cells (PBMCs) were isolated by density gradient centrifugation using Ficoll-Paque PLUS (GE Healthcare, Chicago, IL, USA) according to standard procedures.

\section{Labeling of PBMCs with CellTrace Violet proliferation dye}

To measure proliferation by flow cytometry, PBMCs were labelled with CellTrace Violet (CTV; Invitrogen ${ }^{\mathrm{TM}}$, Life Technologies Europe BV, Bleiswijk, the Netherlands) by resuspending $5 \times 10^{6}$ cells $/ \mathrm{ml}$ in PBS with $5 \mu \mathrm{MCTV}$ and incubation for $20 \mathrm{~min}$ at RT, while the

Table I. Vaccination history of the laying hens. HVT = herpesvirus of turkeys; NDV = Newcastle disease virus; $F=$ fusion protein; IBDV = infectious bursal disease virus; $V P 2=$ minor capsid protein VP2; MDV = Marek's disease virus; IBV = infectious bronchitis virus; $S$. = Salmonella; TRT = turkey rhinotracheitis; $\mathrm{AEV}=$ avian encephalomyelitis virus; $\mathrm{EDSV}=$ egg-drop syndrome virus; $E$. = Escherichia; $P .=$ Pasteurella; ILTV = infectious laryngotracheitis virus.

\begin{tabular}{|l|l|l|}
\hline Age & Vaccine & Method \\
\hline \multirow{3}{*}{ Day I } & Live recombinant HVT + NDV F and IBDVVP2 (HVP360) & Injection \\
\cline { 2 - 3 } & MDV (Rispens) & Injection \\
\cline { 2 - 3 } & Live IBV (stain 4-9I) & Spray course \\
\cline { 2 - 3 } & Live IBV (stain Ma5) & Spray course \\
\cline { 2 - 3 } & Eimeria spores & Spray course \\
\hline Day 7 & Live S. enteritidis and S. typhimurium & Drinking water \\
\hline Day 37 & Live NDV & Spray \\
\hline Day 48 & Live IBV (stains HI20 and D274) & Spray \\
\hline Day 50 & Live S. enteritidis and S. typhimurium & Drinking water \\
\hline Day 56 & Live TRT & Spray \\
\hline Day 69 & Live IBV (stain QX) & Spray \\
\hline Day 70 & Live AEV & Drinking water \\
\hline Day 74 & Inactivated TRT, IBV (strains M4I, 249g), EDSV and NDV & Injection \\
\cline { 2 - 3 } & Inactivated E. coli & Injection \\
\cline { 2 - 3 } & Live ILTV & Eye drop \\
\cline { 2 - 3 } & Inactivated P. multicoca & Injection \\
\hline \multirow{3}{*}{ Day I05 } & Live IBV (stain 4-9I) & Spray course \\
\cline { 2 - 3 } & Live IBV (stain Ma5) & Spray course \\
\hline Day II5 & Live S. enteritidis and S. typhimurium & Drinking water \\
\hline Day 232 & Inactivated IBV (strain M41, D274) and NDV & Injection \\
\hline
\end{tabular}


cell suspension was mixed by gentle shaking every $5 \mathrm{~min}$. Next, unbound dye was quenched by addition of $5 \mathrm{ml}$ RPMI-I 640 cell culture medium supplemented with GlutaMAX ${ }^{\mathrm{TM}}-\mathrm{I}$, phenol red, HEPES, $10 \%$ fetal bovine serum and $50 \mathrm{U} / \mathrm{ml}$ of penicillin - streptomycin (all from Gibco ${ }^{\mathrm{TM}}$, Life Technologies Limited, Paisley, UK) for every $\mathrm{ml}$ of CTV staining solution and incubated for $5 \mathrm{~min}$ at room temperature. Cells were centrifuged for $5 \mathrm{~min}$ at 400x $\mathrm{g}$ and resuspended at $2.5 \times 10^{6}$ cells $/ \mathrm{ml}$ in X-VIVO I 5 cell culture medium (Lonza) with $50 \mathrm{U} / \mathrm{ml}$ penicillin and streptomycin, and $50 \mu M$ 2-mercaptoethanol (Sigma-Aldrich, Saint Louis, MO, USA). The PBMCs $\left(5 \times 10^{5}\right)$ were seeded in $200 \mu \mathrm{l} /$ well in 96 wells round-bottom cell culture plates (Costar ${ }^{\mathrm{TM}}$, Corning B.V. Life Sciences, Amsterdam, the Netherlands).

\section{Ex vivo stimulation}

PBMC were stimulated ex vivo with $2 \mu \mathrm{l} / \mathrm{ml}$ formaldehyde-inactivated IBV and NDV dissolved in allantoic fluid (MSD Animal Health, Boxmeer, the Netherlands), collected from infected embryonated chicken eggs. In addition, cells were stimulated with $2 \mu \mathrm{l} / \mathrm{ml}$ bivalent vaccine (MSD Animal Health), containing inactivated IBV M4I and NDV clone 30 antigens, and emulsified (water-in-oil) mineral oil as adjuvant. As a negative control, $2 \mu \mathrm{l} / \mathrm{ml}$ allantoic fluid without inactivated viruses (MSD Animal Health) was added to the PBMCs. As a positive control, PBMCs were stimulated with I $\mu \mathrm{g} / \mathrm{ml}$ mouse anti-chicken CD3, I $\mu \mathrm{g} / \mathrm{ml}$, mouse antichicken CD28 (both from SouthernBiotech, Birmingham, AL, USA) and 1:50 conditioned medium from the supernatant of a COS-7 cell culture transfected with a pcDNAI vector (Invitrogen ${ }^{\mathrm{TM}}$ ) encoding for recombinant chicken IL-2 (kind gift from prof. Pete Kaiser and dr. Lisa Rothwell) [13]. The plates containing the cells were wrapped in aluminum foil and incubated for 4 days at $41^{\circ} \mathrm{C}$ and $5 \% \mathrm{CO}_{2}$.

\section{Flow cytometric assessment of proliferation}

After incubation, cells were resuspended and transferred to a 96 wells V-bottom plate, centrifuged for $3 \mathrm{~min}$ at 400x g, and stained in $30 \mu \mathrm{l}$ PBS with ViaKrome 808 viability dye (Beckman Coulter Inc., Brea, CA, USA) for 20 min at $4^{\circ} \mathrm{C}$ in the dark. The cells were centrifuged for $3 \mathrm{~min}$ at $400 \mathrm{x} g$ and washed once in FACS buffer (PBS containing $0.5 \%$ BSA and $0.005 \% \mathrm{NaN} 3$, both from Sigma-Aldrich). The cells were stained in FACS buffer containing the monoclonal antibodies mouse anti-chicken CD3-PE (clone CT-3, $2 \mu \mathrm{g} / \mathrm{ml}$ ), mouse anti-chicken CD4-FITC (clone CT-4, I.25 $\mu \mathrm{g} / \mathrm{ml}$ ) and mouse anti-chicken CD8 $\alpha$ APC (clone CT-8, $0.25 \mu \mathrm{g} / \mathrm{ml}$ ) (all from SouthernBiotech) for $20 \mathrm{~min}$ at $4^{\circ} \mathrm{C}$ in the dark. Finally, the cells were washed twice in FACS buffer, centrifuged for 3 min at 400x $g$ in between, and resuspended in $100 \mu \mathrm{FACS}$ buffer. From each sample, $80 \mu \mathrm{l}$ (containing at least $1.2 \times 10^{5}$ and an average of $5.1 \times 10^{5}$ cells) was analyzed using a CytoFLEX LX flow cytometer using 375-, 488-, 56I-, 638-, and 808-nm lasers (Beckman Coulter Inc., Brea, CA, USA) and Flowjo Software v. 10.5 (Flowjo LCC, Ashland, OR, USA).

\section{Statistical analysis}

Statistical analysis was performed using GraphPad Prism 7 software (GraphPad Software, La Jolla, CA, USA). The data were tested for the assumptions of normally distributed data using D'Agostino \& Pearson test, which demonstrated that the results of several groups contained skewed data. Next, the results were tested for significant differences between groups using the Friedman test for nonparametric and matched data and Dunn's multiple comparisons test. Differences with $p$-values of $<0.05$ were considered significant. 
(a)

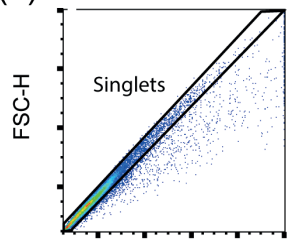

FSC-A

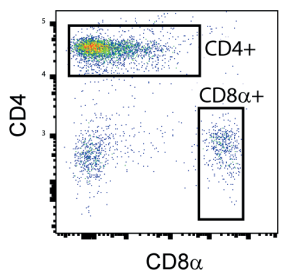

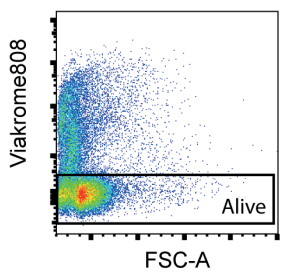
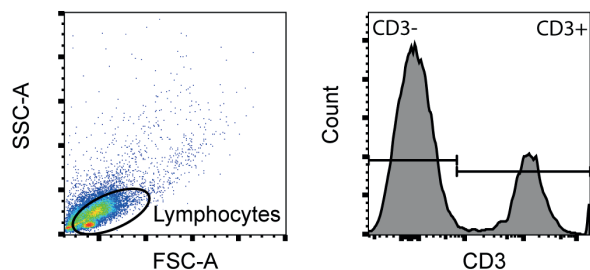

CD3
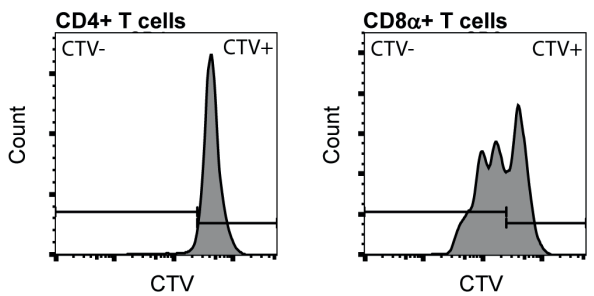

Before vaccination

(b)

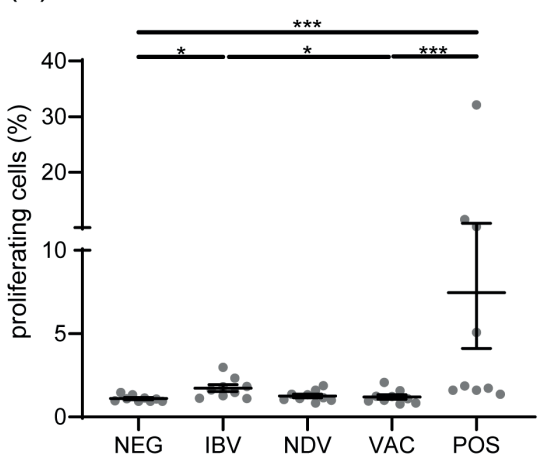

(c) $\mathrm{CD} \mathrm{a}^{+} \mathrm{T}$ cells

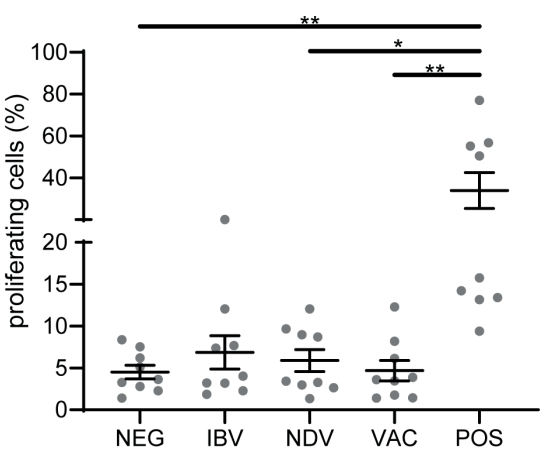

After vaccination

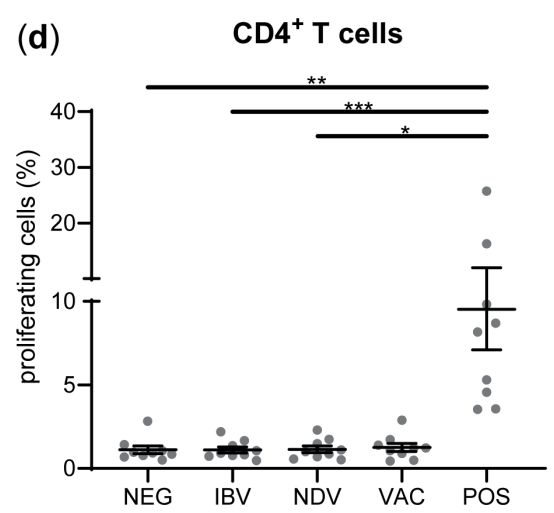

(e)

CD8 $\alpha^{+} T$ cells

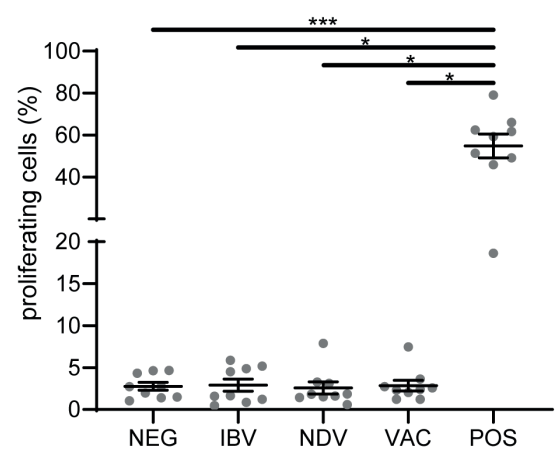


Figure I. T cell proliferation in response to inactivated IBV and NDV, before and after vaccination. PBMCs were isolated from blood of chickens, labelled and analyzed by flow cytometry after 4 days of stimulation. The gating strategy that was used includes gating for single cells (FSC-A/FSC-H), live cells (ViaKrome 808-), lymphocytes (FSC-A/SSC-A), T cells $\left(C D 3^{+}\right)$, and $C D 4^{+} \mathrm{T}$ cells or $\mathrm{CD} 8 \alpha^{+} \mathrm{T}$ cells (a). Finally, the percentages of $\mathrm{CD}^{+}$( $\mathbf{b}$ and $\mathbf{d}$ ) or $\mathrm{CD} 8 \alpha^{+} \mathrm{T}$ cells (c and e) that proliferated at least once were determined by gating the cells that showed reduced fluorescence of CTV. Proliferation was assessed just before (b and $\mathbf{c}$ ) and three weeks after booster vaccination ( $\mathbf{d}$ and $\mathbf{e})$. Stimulation was performed with allantoic fluid as a negative control (NEG), inactivated IBV antigens, inactivated NDV antigens, an inactivated bivalent IBV + NDV vaccine (VAC), and a combination of mouse anti-chicken CD3, mouse anti-chicken CD28 and recombinant chicken IL-2 as a positive control (POS). Every scatter plot shows the results of nine chickens. The error bars show the standard error of the mean. Statistical significance is shown by $*$ for $p<0.05$, ** for $p<0.01$ and $* * *$ for $p<0.001$.

\section{Results}

T cell proliferation after ex vivo stimulation was analyzed for 9 out of 10 chickens, since the amount of blood from I chicken was not sufficient to isolate the number of cells required to perform the assay. Proliferation of $\mathrm{CD}^{+}$and $\mathrm{CD}^{+} \mathrm{T}$ cells was analyzed by flow cytometry. The gating strategy used to determine the percentages of proliferating CD4 $4^{+} \mathrm{T}$ cells and CD8 $\alpha^{+} \mathrm{T}$ cells is shown in Figure Ia.

Before vaccination, ex vivo stimulation with allantoic fluid as negative control resulted in I. I\% (standard error of the mean: $\pm 0.1 \%$ ) proliferating $C D 4^{+} \mathrm{T}$ cells (Figure I b) and $4.5 \%$ $( \pm 0.1 \%)$ proliferating CD $8 \alpha^{+}$T cells (Figure Ic). T cell proliferation significantly increased after stimulation with the positive control, both for $\mathrm{CD}^{+} \mathrm{T}$ cells $(7.5 \% \pm 3.3 \%)$ and $\mathrm{CD} 8 \alpha^{+}$ $\mathrm{T}$ cells $(34 \% \pm 8.6 \%)$. The percentage of proliferating $\mathrm{CD}^{+} \mathrm{T}$ cells increased significantly to $1.7 \%( \pm 0.2 \%)$ after stimulation with IBV antigens, compared to the negative control. Proliferation of CD8 $\alpha^{+} \mathrm{T}$ cells only tended to increase upon stimulation with IBV antigens, which resulted in $6.9 \%( \pm 2.0 \%)$ proliferating cells. Upon stimulation with inactivated NDV antigen or the inactivated IBV + NDV vaccine, no significant changes in proliferation were observed.

Three weeks after the booster vaccination with the inactivated vaccine, stimulation with allantoic fluid as negative control resulted in $1.1 \%( \pm 0.2 \%)$ proliferating $\mathrm{CD}^{+} \mathrm{T}$ cells (Figure Id) and $2.8 \%( \pm 0.5 \%)$ proliferating $C D 8^{+} \mathrm{T}$ cells (Figure Ie). Again, stimulation with the positive control resulted in a significant increase in the proliferation of CD4 ${ }^{+}$ T cells $(9.5 \% \pm 2.4 \%)$ and $C D 8 \alpha^{+} T$ cells $(55 \% \pm 5.6 \%)$. In contrast to before vaccination, no significant changes were observed in the proliferation of $\mathrm{CD}^{+} \mathrm{T}$ cells and CD8 $\alpha^{+} \mathrm{T}$ cells upon stimulation with inactivated IBV antigens. Moreover, no significant changes were observed in the proliferation of T cells upon stimulation with inactivated NDV antigens or the inactivated IBV + NDV vaccine.

Taken together, although $\mathrm{T}$ cell proliferation was readily observed upon polyclonal stimulation and, though to a lower extent, after IBV-specific stimulation before vaccination, neither IBV-specific nor NDV-specific proliferation was increased 3 weeks by the booster vaccination. 


\section{Discussion}

The aim of the present study was to evaluate ex vivo proliferation of $\mathrm{T}$ cells isolated from the blood of laying hens vaccinated against IBV and NDV as an indication for the suitability of $T$ cell proliferation assays for routine vaccine quality control. Since the laying hens of the current study were previously subjected to a standard vaccination regimen, the conditions were similar to the field situation in which application of inactivated (booster) vaccines is preceded by that of live attenuated vaccines to prime the immune response early in life. The set-up of the proliferation assay used in this study was previously optimized and used to demonstrate the induction of Salmonella enteritidis-specific T cells after infection (Meijerink et al., in preparation).

We observed $\mathrm{T}$ cell proliferation upon ex vivo stimulation with inactivated IBV antigen in PBMCs from 33-weeks-old layers exposed to a standard vaccination regimen. Unexpectedly, this response was no longer observed three weeks after an additional booster vaccination with an inactivated IBV/NDV vaccine. A previous study using 3-week-old specific-pathogenfree (SPF) laying hens showed that vaccination with a single inactivated IBV vaccine resulted in a small increase in T cell proliferation up to 33 days [12]. Similar results were obtained after vaccination chickens with a live attenuated vaccine against IBV followed by an inactivated vaccine after three moths [12]. Thus, vaccination with an inactivated vaccine against IBV results in a limited induction of CMI. In contrast, infection with virulent IBV leads to a strong induction of CMI between 2 and 6 weeks after infection, as demonstrated by $\mathrm{T}$ cell proliferation upon stimulation with inactivated IBV ex vivo [I4]. Eighteen weeks before the chickens entered the current study they were vaccinated with a live attenuated vaccine for IBV. Likely, the IBV-specific T cell response that we observed ex vivo before vaccination at 33 weeks resulted from previous vaccination with live attenuated vaccines. Three weeks later, at 36 weeks, the frequency of IBV-specific T cells might have declined to undetectable levels. This rapid decline in IBV-specific T cells after vaccination or infection has been observed by others before [12].

Ex vivo stimulation of T cells with NDV or a bivalent vaccine against IBV and NDV did not result in increased $\mathrm{T}$ cell proliferation. Previous studies that evaluated $\mathrm{CMI}$ in chickens vaccinated with NDV showed different outcomes. Some indicated $\mathrm{CMI}$ in chickens that received live attenuated vaccines $[8,9]$ or in chickens that were vaccinated with a combination of live attenuated and inactivated vaccines $[8,10]$, however, without specifically looking at the effect of the inactivated vaccine. The induction of $\mathrm{T}$ cell proliferation was shown to be highly dependent on MHC haplotype as well as highly variable even within haplotypes [10]. Another study evaluated the effect of vaccination of naive chickens with an inactivated vaccine against NDV between I and 4 weeks after vaccination, but observed only limited interferon- $\gamma$ production after 4 weeks and no $\mathrm{T}$ cell proliferation ex vivo [II]. Although the authors suggested that more than a single vaccination might be required to detect CMI, we were not able to demonstrate $\mathrm{CMI}$ in chickens that were vaccinated multiple times against NDV.

This study shows that although $\mathrm{T}$ cell proliferation was readily observed upon polyclonal stimulation, it remains difficult to detect proliferation of low frequencies of IBV- or NDVspecific (memory) $\mathrm{T}$ cells upon ex vivo stimulation. Further optimization of the assay 
methods may be needed to enable measurement of low frequencies of antigen-specific $T$ cells by proliferation. During optimization of the $T$ cell proliferation assay, we did already assess whether antigen-presenting cells were present in the culture, which was confirmed by the presence of cells expressing major histocompatibility complex class II at both the start and the end of the culture (not shown). Secondly, like in a previous study we showed that X-VIVO I5 culture medium, the medium that was used in this study, is suitable to perform chicken $\mathrm{T}$ cell proliferation assays [8]. Thirdly, the 4-day stimulation period that was used in the $T$ cell proliferation assay of the current study was relatively short, though in line with a previous study showing CMI after vaccination against NDV [10]. Since a previous study has demonstrated that the proliferative capacity of $T$ cells declines with ageing [I2], the age of the chickens used in our study might explain our difficulty to detect CMI by $\mathrm{T}$ cell proliferation. Furthermore, doses of the antigens and the vaccine used for stimulation might have been insufficient to induce proliferation of antigen-specific $\mathrm{T}$ cells, but higher concentrations were found to be toxic for the cells in optimization experiments prior to this study. Purified or recombinant antigens could be used in future studies to reduce toxicity. Alternatively, an explanation can be that the inactivated poultry vaccine against IBV and NDV does not strongly induce CMI, but rather depends on the induction of antibodymediated responses. This needs to be addressed by future studies.

Based on the results of this study, the presented $\mathrm{T}$ cell proliferation, assay, even though functioning properly after polyclonal stimulation, was not sensitive enough for routine quality control of inactivated poultry vaccines for IBV and NDV. Future studies with slightly younger chickens or antigens of higher dose are warranted to potentially optimize the assay.

\section{Acknowledgements}

We thank the animal caretakers of the division Farm Animal Health, department Population Health Sciences, Faculty of Veterinary Medicine, Utrecht University for taking great care of the animals and F.C. Velkers of the same division for her advice and for providing the animals that were part of this study. Furthermore, we thank N. Meijerink of the division Infectious Diseases and Immunology, department Biomolecular Health Sciences, Faculty of Veterinary Medicine, Utrecht University for her help during PBMC isolation. 


\section{References}

I. Peebles ED. In ovo applications in poultry: A review. Poult Sci (2018) 97(7):2322-2338. doi:10.3382/ps/pey08I

2. Bande F, Arshad SS, Bejo MH, Moeini H, Omar AR. Progress and challenges toward the development of vaccines against avian infectious bronchitis. J Immunol Res (2015) 2015:424860. doi: $10.1155 / 2015 / 424860$

3. Darbyshire JH, Peters RW. Humoral antibody response and assessment of protection following primary vaccination of chicks with maternally derived antibody against avian infectious bronchitis virus. Res Vet Sci (1985) 38(I):I4-2I. doi:I0.1016/s0034-5288(I8)3I84I-I

4. Stone HD, Brugh M, Hopkins SR, Yoder HW, Beard CW. Preparation of inactivated oilemulsion vaccines with avian viral or Mycoplasma antigens. Avian Dis (1978) 22(4):666-674. doi: $10.2307 / 1589643$

5. Cessi D, Nardelli L. Vaccination against newcastle disease: Efficacy of an oil emulsion vaccine. Avian Pathol (1974) 3(4):247-253. doi: I0.1080/03079457409353838

6. Seo SH, Pei J, Briles WE, Dzielawa J, Collisson EW. Adoptive transfer of infectious bronchitis virus primed alphabeta $T$ cells bearing CD8 antigen protects chicks from acute infection. Virology (2000) 269(I):183-189. doi:I0.1006/viro.2000.02II

7. Marino OC, Hanson RP. Cellular and humoral response of in ovo-bursectomized chickens to experimental challenge with velogenic Newcastle disease virus. Avian Dis (1987) 3I(2):293-30I. doi: $10.2307 / 1590875$

8. Dalgaard TS, Norup LR, Rubbenstroth D, Wattrang E, Juul-Madsen HR. Flow cytometric assessment of antigen-specific proliferation in peripheral chicken $T$ cells by CFSE dilution. Vet Immunol Immunopathol (2010) I38(I-2):85-94. doi:I0.1016/j.vetimm.2010.07.010

9. Dalgaard TS, Norup LR, Pedersen AR, Handberg KJ, Jørgensen PH, Juul-Madsen HR. Flow cytometric assessment of chicken $\mathrm{T}$ cell-mediated immune responses after $\mathrm{Newcastle}$ disease virus vaccination and challenge. Vaccine (20I0) 28(28):4506-45I4. doi:I0.1016/j.vaccine.20 I0.04.044

10. Norup LR, Dalgaard TS, Pedersen AR, Juul-Madsen HR. Assessment of Newcastle disease-specific $T$ cell proliferation in different inbred MHC chicken lines. Scand J Immunol (20II) 74(I):23-30. doi: I0.1 I II/j.1365-3083.20II.02534.x

II. Lambrecht B, Gonze M, Meulemans G, Van Den Berg TP. Assessment of the cell-mediated immune response in chickens by detection of chicken interferon- $\gamma$ in response to mitogen and recall Newcastle disease viral antigen stimulation. Avian Pathol (2004) 33(3):343-350. doi: I0.1080/03079450420002203/8

12. Timms LM, Bracewell CD. Cell mediated and humoral immune response of chickens to inactivated oil-emulsion infectious bronchitis vaccine. Res Vet Sci (1983) 34(2):224-230. doi:10.1016/s00345288(18)32264-I

13. Sundick RS, Gill-Dixon C. A cloned chicken lymphokine homologous to both mammalian IL-2 and IL-I5. J Immunol (1997) I59(2).

14. Timms LM, Bracewell CD, Alexander DJ. Cell mediated and humoral immune response in chickens infected with avian infectious bronchitis. Br Vet J (1980) 136(4):349-356. doi:https://doi. org/I0.1016/S0007-1935(17)32237-6 


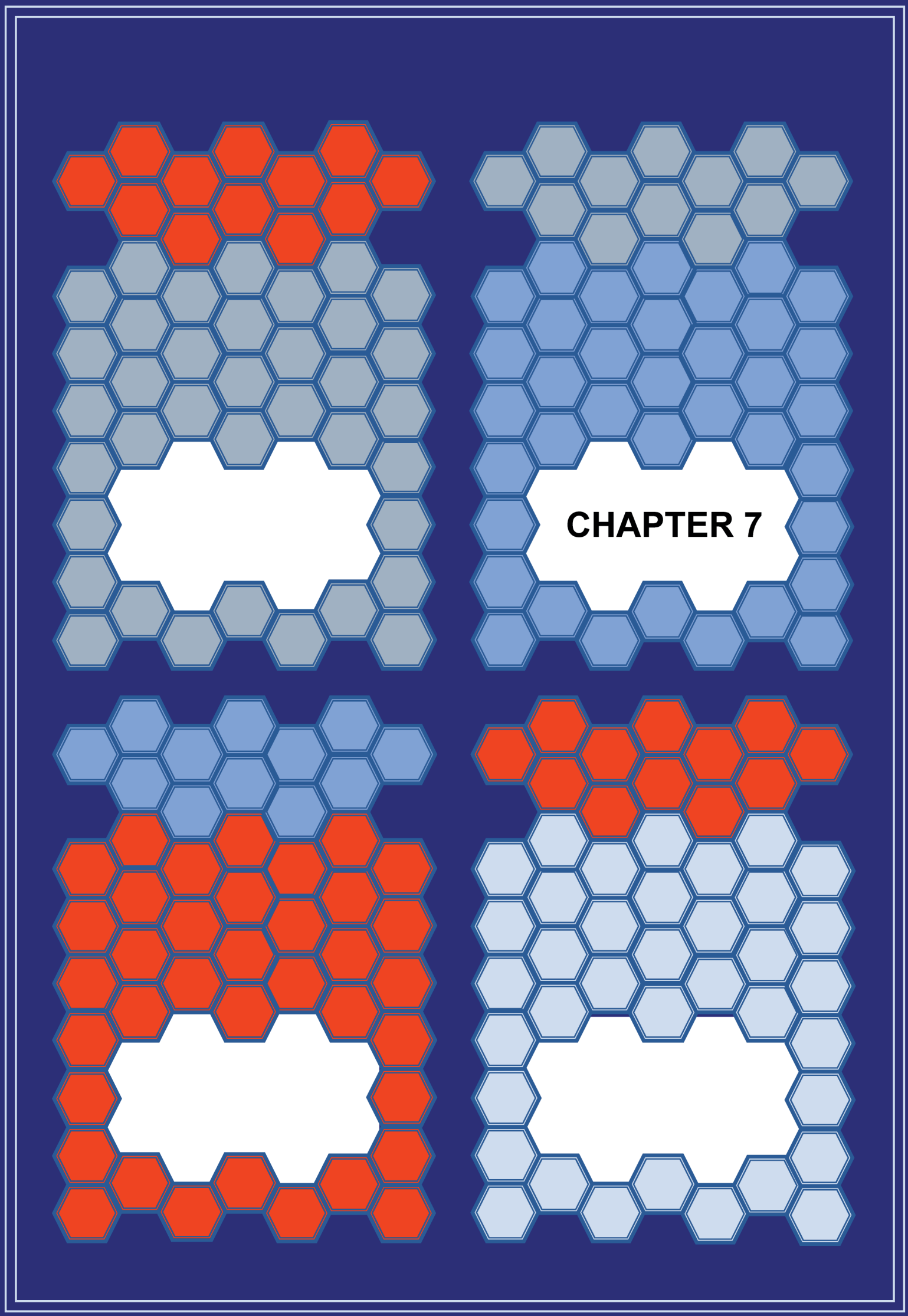




\section{Overcoming scientific barriers in the transition from in vivo to non-animal batch testing of human and veterinary vaccines}

Robin H.G.A. van den Biggelaar', Marcel H.N. Hoefnagel ${ }^{2}$, Rob J. Vandebriel ${ }^{3}$, Arjen Sloots ${ }^{4}$, Coenraad F.M. Hendriksen ${ }^{4}$, Willem van Eden', Victor P.M.G. Rutten $^{1,5}$ and Christine A. Jansen ${ }^{1,6}$

' Division of Infectious Diseases and Immunology, Department of Biomolecular Health Sciences, Faculty of Veterinary Medicine, Utrecht University, Utrecht, the Netherlands

${ }^{2}$ Quality Department, Medicines Evaluation Board, Utrecht, the Netherlands

${ }^{3}$ Centre for Health Protection, National Institute for Public Health and the Environment (RIVM), Bilthoven, the Netherlands

${ }^{4}$ Intravacc (Institute for Translational Vaccinology), Bilthoven, the

Netherlands

${ }^{5}$ Department of Veterinary Tropical Diseases, Faculty of Veterinary Science, University of Pretoria, Pretoria, South Africa

${ }^{6}$ Cell Biology and Immunology Group, Department of Animal Sciences, Wageningen University and Research, Wageningen, the Netherlands

\section{(Submitted)}


Chapter 7 | The transition to non-animal batch testing of human and veterinary vaccines

\section{Abstract}

Before their release on the market, vaccine batches are assessed for quality to evaluate whether they meet the product specifications. Batch tests of many human and veterinary vaccines, and in particular of inactivated and toxoid vaccines, still largely rely on in vivo methods. Improved vaccine production processes, ethical concerns, and suboptimal performance of some in vivo tests have led to a global intent to develop in vitro alternatives. Currently, some in vivo potency and safety tests have been replaced by in vitro assays. However, for many in vivo tests such alternatives are not yet available or poorly implemented. In this review, we focused on scientific constraints that need to be overcome for replacement of in vivo batch tests of vaccines, as well as potential solutions. Topics include the critical quality attributes of vaccines that require testing, the use of cell-based assays to mimic relevant aspects of in vivo vaccine-induced immune responses, how difficulties with testing adjuvanted vaccines in vitro can be overcome, the use of altered batches to validate new in vitro test methods, and how cooperation between different stakeholders is key to move the transition forward. 


\section{Background}

Vaccination is a cost-effective strategy to prevent infectious diseases among humans and livestock. Vaccines are biologicals that are used to immunize large groups of healthy individuals and they may be subject to inherent batch-to-batch variation, which is why newly produced vaccine batches require quality assessment before being released to the market. Vaccines have traditionally been generated using trial and error approaches involving in vivo experiments [I]. Subsequently produced batches of such established vaccines are often still tested using in vivo methods to confirm that the quality of the new vaccine batch meets the specifications as defined in the marketing authorization. These specifications concern purity (i.e., freedom from extraneous matter), potency (i.e., the capacity of a vaccine batch to exert its effect), safety (i.e., relative freedom of harmful effects) and efficacy (i.e., effect of vaccination on the target species/population under ideal circumstances) of the vaccine [2]. Over one million animals were used for batch potency and safety testing of medicinal products in the EU in 2017, which corresponds to approximately $12 \%$ of total animal use for scientific purposes in the EU [3]. Nowadays, there are several reasons to move away from the use of these in vivo potency and safety tests to assess the quality of vaccine batches. Firstly, significant improvements in and standardization of the vaccine production process, adherence to good manufacturing practice (GMP) guidelines and in-process controls have resulted in less batch-to-batch variation and a lower risk of producing unsafe or ineffective products [4]. Secondly, the use of large numbers of animals in experiments that may inflict pain and distress is not in line with the ethics of contemporary research and the 3Rs principles of Replacement, Reduction and Refinement [5-7]. Thirdly, the relevance of some in vivo tests is disputed because the test results show high variability and poor reproducibility [7-12]. Fourthly, the use of animal models is expensive, time consuming and risky for personnel when models involve exposure to viable pathogenic organisms [7].

The consistency approach was proposed as a strategy to enable the transition of in vivo to in vitro batch testing of vaccines [13,14]. This approach is based on the principle that quality is the consequence of consistent production of subsequent batches monitored by a GMP quality system [15]. The evaluation of a number of pre-defined vaccine parameters using in vitro physicochemical, immunochemical and cell-based test methods should demonstrate that final batches are of consistent quality, making the use of in vivo tests unnecessary.

Major steps have been taken to promote the use of in vitro alternatives for vaccine batch testing, including the creation of a legal and logistic framework. For instance, Directive 2010/63/EU on the protection of animals used for scientific purposes, which includes regulatory testing of vaccines, states "The use of animals for scientific or educational purposes should only be considered where a non-animal alternative is unavailable" and thus promotes the use of novel in vitro test methods [16]. In addition, a general chapter on the "Substitution of in vivo methods by in vitro methods for the quality control of vaccines" has been incorporated in the European Pharmacopoeia to provide guidance on the implementation of the consistency approach in vaccine quality testing (5.2.I4; [17]). Bodies like the Biological Standardisation Programme of the European Directorate for the Quality of Medicines \& Healthcare (EDQM), which facilitates multi-center validation studies [I8], and the European Centre for Validation of Alternative Methods (ECVAM), which acts as a reference laboratory of the European Union [16], provide the logistic framework needed to 
validate novel in vitro methods. Meanwhile, some in vivo tests of vaccines have been replaced by in vitro alternatives, whereas others are no longer used as will be discussed further below.

In September 20I5, industrial, regulatory and scientific experts gathered for a workshop in the Netherlands to identify drivers and barriers for the implementation of the consistency approach, of which the results were published [15]. Newer generation vaccines, including recombinant subunit vaccines (e.g. human papillomavirus and hepatitis B) or conjugate vaccines (e.g. Haemophilus influenzae type b, meningococcus and pneumococcus) are well defined and can be evaluated for quality using in vitro methods $[17,19,20]$, which is why the workshop focused on established live attenuated, inactivated and toxoid vaccines that were in part still tested for quality using in vivo methods. Identification and development of in vitro methods that provide alternatives to currently used in vivo batch tests of vaccines is hampered by several scientific and other constraints including the following points [15]:

- It is difficult to mimic vaccine-induced immune responses using in vitro test methods.

- Measuring vaccine properties of adjuvanted vaccines is complex.

- There is a need to create subpotent formulations for method validation due to a lack of appropriate non-compliant batches to test (and validate) in vitro methods.

- Research into in vitro test methods needs to be prioritized and financed based on number of test animals, level of severity, and performance of the in vivo test.

- Knowledge about the critical quality attributes of vaccines and critical process parameters of vaccine production is often limited.

In the present review we will discuss the scientific barriers that have been overcome since the workshop or still need to be dealt with to enable a complete transition from in vivo to in vitro vaccine quality testing.

\section{Current repertoire of in vitro vaccine quality tests}

Despite the constraints described above [13], several human and veterinary vaccines already underwent the transition from in vivo to in vitro batch testing (Figure I). These vaccines could provide a roadmap to guide the transition to in vitro for the vaccines that are currently still tested for potency and safety using in vivo methods.

For live attenuated vaccines, comprising bacteria or viruses, the use of in vivo batch tests is in general not demanded in the European Pharmacopoeia (Supplementary Table I) [I7]. Due to their replicative nature, live attenuated vaccines can be tested for potency by viral or bacterial titration. Some live attenuated viral vaccines (e.g. influenza virus and viral poultry vaccines) are propagated and titrated on embryonated chicken eggs when suitable cell lines are not available. These chicken embryos are not regarded as laboratory animals in view of the European act on the protection of animals used of scientific purposes (Directive 20 10/63/ EU) is not applicable to fetal stages of birds [16]. However, the embryos may experience pain [2I] and cell-based alternatives to propagate and titrate these viruses are therefore desirable whenever available. Moreover, virus propagation through cell lines allows faster upscaling during epidemics and is better standardized than propagation through eggs [22]. Finally, the transportation of chicken eggs may be prohibited by governments during avian influenza outbreaks. 


\section{(a)}

Potency tests

Newcastle disease
bovine and canine
leptospirosis
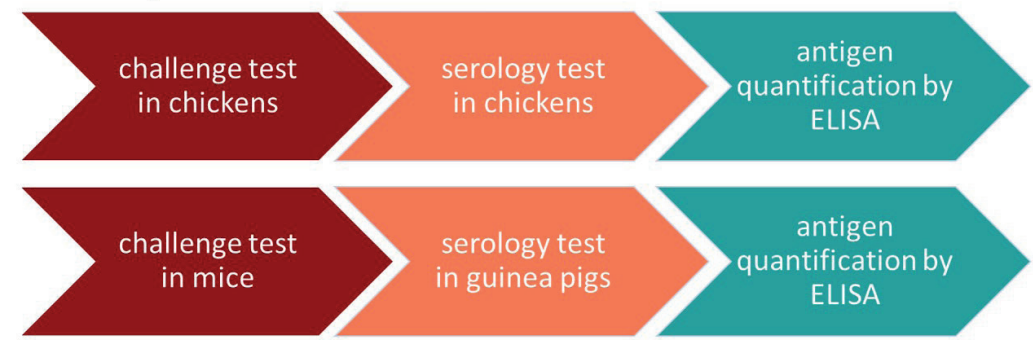

rabies
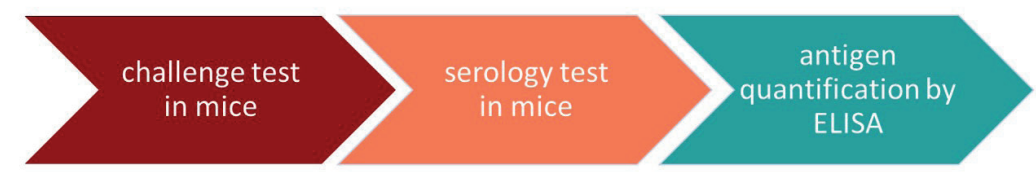

hepatitis $A / B$
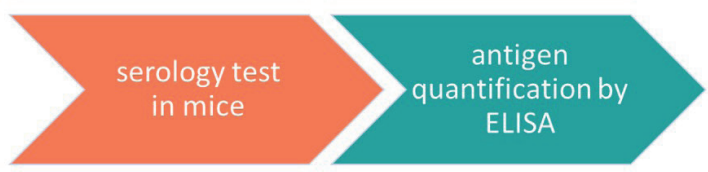

inactivated
poliomyelitis

\section{(b)}

pertussis

diphtheria

clostridials

pyrogenicity tests

\section{Safety tests}

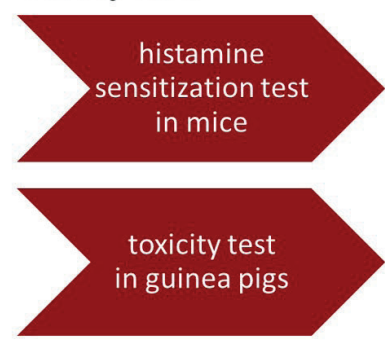

serology test

in rats, chickens

or guinea pigs

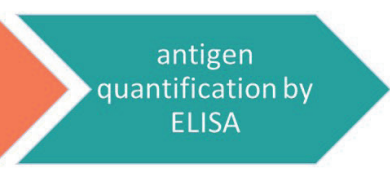

pyrogenicity tests
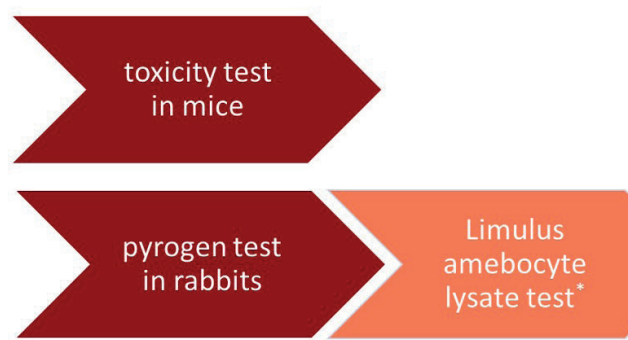
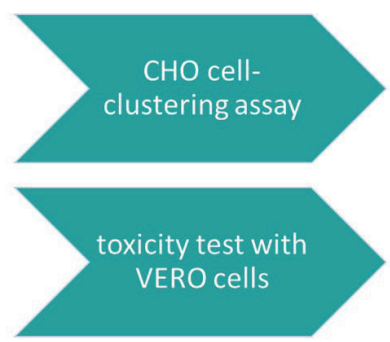

toxicity test with VERO cells
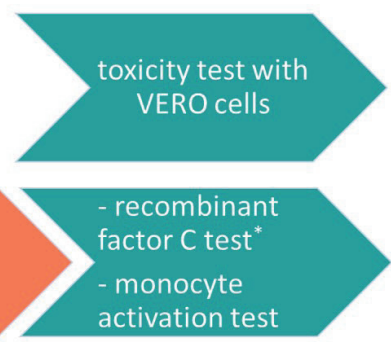

* These alternatives only test for endotoxin pyrogens.

Figure I. For several established inactivated and toxoid vaccines for human and veterinary use there has already been a transition from in vivo to in vitro batch testing in Europe [5]. Advancements have been made for both potency (a) as well as safety tests (b). Conventional in vivo tests are shown in red. Alternative in vivo tests with reduced and refined use (i.e., less animals and less discomfort) of animals are shown in orange. Alternative in vitro tests are shown in green. 
Unlike live attenuated vaccines, inactivated vaccines and toxoid vaccines mostly rely on vaccination-challenge or vaccination-serology potency tests, unless antigen quantification methods are available as in vitro alternatives (Supplementary Table I). These antigen quantification methods may be physiochemical or immunochemical assays that are specific for one or more dominant antigens of the vaccines. In particular, the development of enzymelinked immunosorbent assays (ELISAs) for antigen quantification has been successful for the replacement of in vivo potency tests of several vaccines in Europe, including those against Newcastle disease in poultry [23], foot-and-mouth disease in cattle [24], leptospirosis in cattle and dogs (monographs 0447 and 1939; [17]), rabies [25] in both animals and humans, and hepatitis A [26] and B [20] in humans (Figure Ia). Other ELISAs for antigen quantification have shown to be successful in determining the potency of some but not all inactivated vaccines and toxoid vaccines, but these are not yet approved by the regulatory authorities in Europe. These include tests for infectious bronchitis virus and infectious bursal disease virus vaccines for poultry [27], furunculosis vaccines for salmonids [28], tetanus vaccines for human [29] and veterinary [30] use, and diphtheria vaccines for human use $[31,32]$. In addition to the ELISA, another important antigen quantification method is the immunodiffusion test, which is used to determine the content of hemagglutinin antigens of inactivated or subunit influenza vaccines [33]. For the whole-cell and acellular pertussis vaccines, there have been efforts to develop in vitro ELISA [34], Luminex [35], cell-based $[36,37]$ and proteomic assays [38] as alternatives.

With respect to safety, alternative in vitro methods have also become available in Europe (Figure I b). Originally, vaccines were tested for pyrogenicity using the Rabbit Pyrogen Test (RPT), in which temperature changes in rabbits are evaluated after injection of a vaccine. To specifically quantify endotoxin pyrogens, comprising bacterial lipopolysaccharides (LPS), the Limulus Amebocyte Lysate (LAL) test is used. Although the LAL test is legally not regarded as an animal test, 100,000 horseshoe crabs die annually due to the bleeding [39], which impacts the survival of the species classified as threatened by the International Union for Conservation of Nature [40]. Nowadays, the blood clotting factor C ( $r F C)$ from the horseshoe crab is commercially available as a recombinant protein [39], but the rFC test has been implemented to a limited extent despite its proven efficacy [39] and its adoption in the European Pharmacopoeia as alternative reagent in endotoxin tests (general text 2.6.14, 2.6.32 and 5.I.10; [17]). The Monocyte Activation Test (MAT) was developed to detect both endotoxin and non-endotoxin pyrogens [4I-43]. In this test human whole blood or (cryopreserved) PBMCs are stimulated with the vaccine of interest after which the production of pro-inflammatory cytokines (TNF, IL-I $\beta$ and IL-6) is assessed in ELISAs. The European Pharmacopoeia states that whenever possible and after product-specific validation, the RPT test should be replaced by the MAT (general text 2.6.8; [17]). To justify the use of the LAL test or the rFC test as alternatives for the RPT test, a risk assessment using the MAT is recommended to rule out the presence of any non-endotoxin pyrogens in a vaccine (general text 5.I.10). These statements promote the use of the MAT as a safety test for pyrogenicity or bacterial endotoxins. The MAT test was recently optimized for pyrogenicity testing of the Encepur vaccine against tick-borne encephalitis [4I].

To test for residual toxicity of toxoid vaccines in vitro alternatives have been developed including the Chinese hamster ovary $(\mathrm{CHO})$ cell clustering assay as an alternative to the in vivo Histamine Sensitization Test (HIST) for acellular pertussis vaccines [44]. In addition, 
VERO cells toxicity assays to use instead of mouse toxicity tests for veterinary Clostridium septicum vaccines are currently being validated [45]. Finally, the binding and cleavage (BINACLE) assay evaluates residual tetanus toxicity of human tetanus vaccines [46], as an alternative for the currently used test in guinea pigs, and is currently being validated as part of the European Biological Standardisation Programme.

In summary, although the quality of many inactivated vaccines and toxoid vaccines is still tested with in vivo potency tests, an increasing number of in vitro alternatives are being developed to achieve their replacement. These alternatives include immunochemical assays to quantify antigen, but also tests for other critical properties of vaccines, as will be discussed in the next section. For safety tests, many in vitro alternatives are already available in Europe and implementation of these methods has now become important to move the transition to in vitro safety testing forward.

\section{Critical quality attributes of vaccines}

Critical quality attributes of vaccines are physical, chemical or microbiological properties of vaccines that should be within certain limits to ensure vaccine quality [47]. The in vitro methods that are currently in place for potency testing of vaccine batches are mainly used to evaluate antigen identity, quantity and integrity (Figure I a). However, antigen quantification methods often depend on the integrity of a single antigen [48], while at population level vaccination efficiency hardly ever depends on a single dominant epitope. Furthermore, the ability of immunochemical assays to measure the relevant epitopes of antigens inactivated with agents like formaldehyde and $\beta$-propiolactone should be validated. The inactivation may alter or hide specific epitopes of antigens and hence affect potency as measured in vitro [48-5I], even when the potency as measured in vivo remains the same [48,50,5I]. The use of a pool of monoclonal antibodies in ELISAs can result in more consistent and standardized quality testing of vaccines, without depending too much on single epitopes [48]. In addition to antigen identity, quantity and integrity characteristics, the potency of vaccines may depend on critical quality attributes like vaccine composition, susceptibility of the antigen to proteolytic degradation, the spatial organization (i.e., three-dimensional structure) of the antigen, or the presence of additional immunostimulatory molecules. A combination of assays addressing these different critical quality attributes may thus be required to sufficiently demonstrate batch-to-batch consistency for some vaccines.

The vaccine composition depends on production processes, and inconsistencies in these processes may affect the potency of the vaccine. For instance, for whole-cell pertussis vaccines it was shown that disturbances of the bacterial culture conditions may results in the downregulation of important virulence proteins [52]. Mass spectrometry-based proteome analysis was proposed as a method to evaluate the whole protein composition of vaccines to detect these disturbances [38]. Another applicability of mass spectrometry is the assessment of the stability of antigens and their susceptibility to enzymatic degradation as an important part of antigen processing by antigen-presenting cells [53]. Inactivation by formaldehyde [54] and heat exposure [55] were shown to affect the degradation kinetics of a model antigen and tetanus toxoid, respectively. The importance of the spatial organization of antigens has been demonstrated for influenza vaccines by showing that during a priming vaccination whole-inactivated influenza vaccines induced higher antibody titers, both in mice 
[56,57] and humans [58], and superior T cell responses in humans [59] as compared to the less reactogenic split vaccines with spatially disrupted antigens [60]. The antigens of the split vaccine lack the proper spatial organization to efficiently induce antibody production [6I]. Similar results have been found for detergent-treated inactivated vaccines against Newcastle disease virus for use in poultry [62]. Finally, the presence of immunostimulatory molecules, which may include exogenous adjuvants or endogenous pathogen-associated molecular patterns (PAMPs), is important for vaccine potency. The contribution of PAMPs to vaccine potency has been demonstrated for both viral (e.g. influenza [57]) and bacterial (e.g. pertussis [36,63]) vaccines. Similar to the antigens, PAMPs can be destroyed or become less accessible by inactivating agents like formaldehyde or $\beta$-propiolactone $[49,64]$. The presence of immunostimulatory molecules can be evaluated using cell-based assays, as discussed in more detail below. The described quality attributes may require additional testing when antigen identity, quantity and integrity characteristics are insufficient to predict the potency of a vaccine.

The previous section included in vitro alternatives to test vaccines for pyrogenicity and to test toxoid vaccines for residual toxicity. Furthermore, toxicity tests that were found to be unnecessary based on historical data have recently been deleted from the European Pharmacopoeia, including abnormal toxicity tests (to detect any unexpected hazards), some specific toxicity tests of human vaccines, and some residual toxicity tests of veterinary vaccines $[65,66]$. However, the BINACLE assay is not suitable for use with adjuvanted tetanus toxoids [46]. In contrast, the porcine actinobacillosis vaccine, porcine progressive atrophic rhinitis vaccine, and tetanus vaccine for veterinary use still require animal-based toxicity tests [17,65]. Moreover, safety tests of the Bacillus Calmette-Guérin vaccine, used to protect against tuberculosis, include the virulent mycobacteria test and the excessive dermal reactivity test, both in guinea pigs [17], to show absence of virulence and excessive reactogenicity, respectively. An alternative safety assay based on the proliferation of lymphocytes from sensitized guinea pigs has been proposed instead of the currently used excessive dermal reactivity test [67]. A vaccine for rabbit hemorrhagic disease still requires a residual live virus safety test in rabbits [17]. Some live attenuated viral vaccines (e.g. smallpox and poliomyelitis) require neurovirulence safety testing in monkeys or transgenic mice [17], although deep sequencing methods have been proposed as an alternative strategy to test these vaccines for genetic instability and to prevent the occurrence of neurovirulent viral mutants $[68,69]$. Recently, a model based on brain cells in a transwell system, named the BBB-Minibrain culture device, was developed as a next step in search for an alternative neurovirulence test [70]. Finally, batch release of pertussis vaccines still requires a test for residual dermonecrotic toxin in mice [17]. Recently, the use of liquid chromatography mass spectrometry to quantify dermonecrotic toxin has been proposed as an alternative in vitro method [7I].

To summarize this section, antigen identity, quantity and integrity can be considered the most important critical quality attributes of inactivated and toxoid vaccines. However, additional quality attributes including vaccine composition, the spatial organization of the antigen, or the presence of additional immunostimulatory molecules may be critical for specific vaccines. Importantly, it is increasingly recognized that one-to-one replacement of an in vivo test for an in vitro test will be difficult and that a combination of assays to demonstrate batch-to-batch consistency may be needed for some vaccines [II,72]. 


\section{Mimicking in vivo vaccine-induced immune responses using in vitro cell-based assays}

The use of immunochemical and physicochemical methods for potency testing are based on evidence showing that consistency of batches, with regard to one or more aspects like antigen identity, antigen quantity, antigen integrity, antigen structure, vaccine composition, and quantity of immunostimulatory molecules will lead to consistent outcomes in vivo. For vaccines that are well defined (e.g. recombinant and subunit vaccines) the use of these methods may indeed be sufficient to guarantee the potency and safety of a vaccine. However, for less defined inactivated and perhaps even toxoid vaccines, additional information on the immunostimulatory capacity of vaccines may be needed to generate sufficient evidence about vaccine quality when in vitro methods are used. Cell-based assays can assess aspects of vaccine-induced immune reactivity and may identify critical quality attributes of vaccine.

An immune response is initiated by innate immune cells (Figure 2; step I), among which professional antigen-presenting cells, including dendritic cells, macrophages and B cells process and present antigen, and in addition express co-stimulatory molecules and release cytokines to activate T cells (Figure 2; step 2). Several methods have been proposed to evaluate vaccine potency in cell-based assays with immune cells using either primary cells or immortalized cell lines [37,73-77]. Primary dendritic cell-based assays use monocytederived dendritic cells (moDCs) [37,75-77] or bone marrow-derived dendritic cells (BMDCs) [74]. Whereas primary cells more closely represent the physiological nature of immune cells, they are collected from animals or human donors, often have a limited lifespan, are available in limited numbers, and may show variable responses due to genetic diversity of the individual donors. Cell lines do not have these disadvantages and are thus being explored for use in a vaccine quality control setting (Table I), even though they may be less representative for the in vivo situation than primary cells. Most cell-based potency assays measure vaccine-induced activation of dendritic cell-, monocyte- and macrophagelike cells by expression of co-stimulatory molecules (e.g. CD40, CD80, CD83, CD86) or pro-inflammatory cytokines. Furthermore, dendritic cells have been used in in vitro antigen degradation assays, in which the susceptibility of antigens for proteolytic degradation is used as a biomarker for immunogenicity [53]. Reporter cell lines like PRR-expressing human embryonic kidney 293 (HEK)-Blue cells have been used to evaluate the immunostimulatory properties of clinical isolates of Bordetella pertussis $[78,79]$ and may also be useful to test vaccines for potency.

Assays with innate immune cells have also been used to evaluate safety aspects of vaccines. Vaccine pyrogenicity can be evaluated by the MAT using monocytic cell lines like the MM6 cell line [86]. Interestingly, the MM6 cell line has been explored for use in in vitro safety tests [8I], as well as potency tests [37], both using secretion of the pleiotropic cytokines IL-I $\beta$ and IL- 6 as readouts. The use of IL-I $\beta$ and IL- 6 as readouts for potency is in line with the role of these cytokines in T cell activation and differentiation [87,88], whereas their use as readouts for safety is in line with their ability to induce fever and other potential side effects of vaccination [8I]. Thus, potency and safety limits of pro-inflammatory cytokines need to be identified beforehand to discriminate between potent and potentially dangerous levels of vaccine-induced immune responses [8I]. 


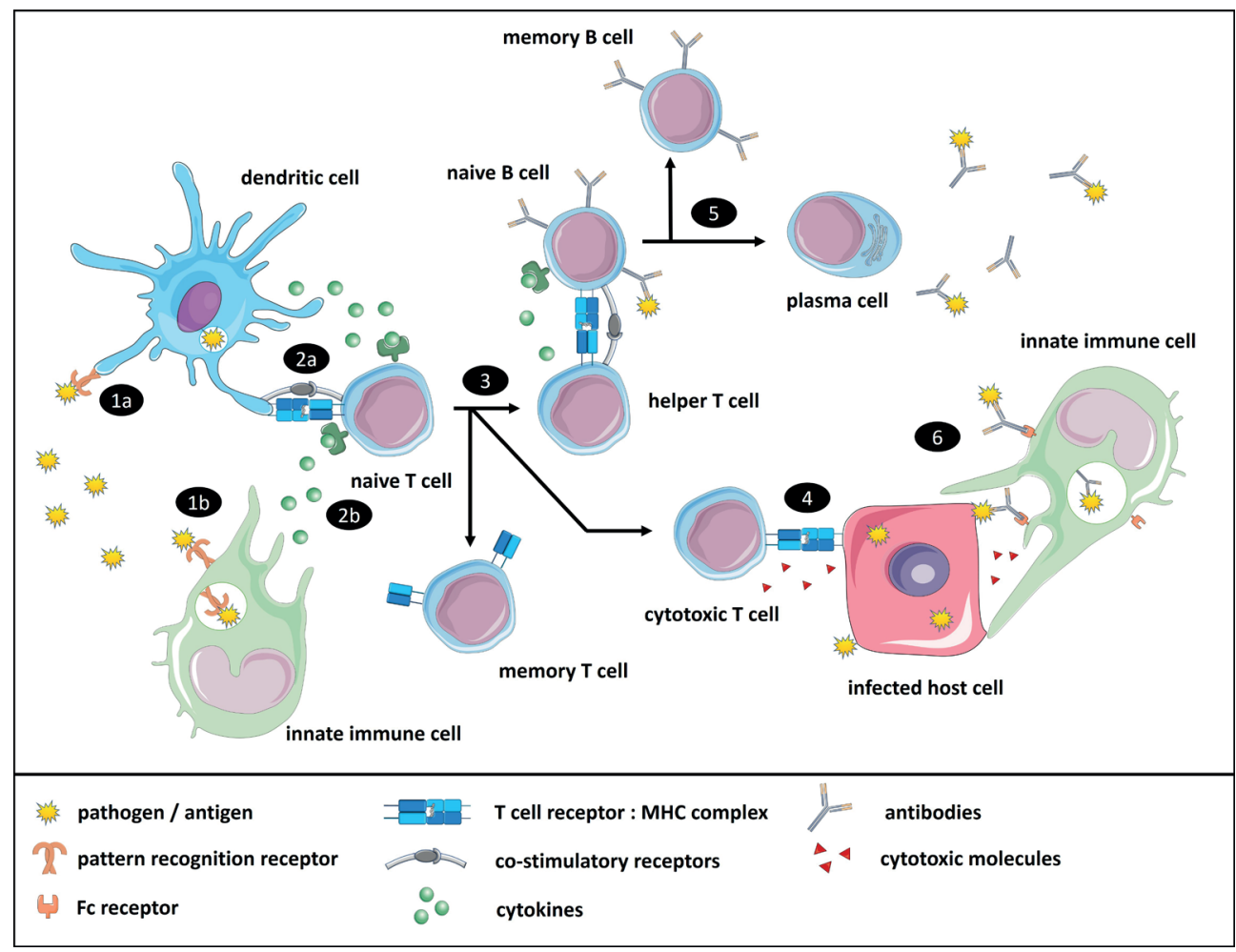

Figure 2. Simplified overview of a vaccine-induced immune response. (I) Innate immune cells recognize pathogen-associated molecular patterns (PAMPs), e.g. bacterial cell wall components or double-stranded RNA that are part of vaccine antigens, through pattern recognition receptors (PRRs). (2) Upon recognition, vaccine antigens are taken up by innate immune cells, and processed for antigen presentation on major histocompatibility complexes (MHC) class I and II to (naive) antigen-specific T cells. Innate immune cells orchestrate the adaptive immune response by releasing cytokines that affect the differentiation of $\mathrm{T}$ cells. (3) As a result, $\mathrm{CD} 4^{+} \mathrm{T}$ cells differentiate into various types of $\mathrm{CD} 4^{+}$ helper $T$ cells $\left(T_{H} 1, T_{H} 2, T_{H} 17, T_{R E G}\right.$ etc.) or memory $T$ cells, whereas $C D 8^{+} T$ cells differentiate into $\mathrm{CD}^{+}$cytotoxic $T$ cells, or memory $T$ cells. (4) Cytotoxic $T$ cells recognize antigen-derived peptides presented on MHC class I molecules of infected host cells that are subsequently killed. (5) Antigenspecific naive $B$ cells that bind antigens through their $B$ cell receptor will endocytose and process the antigens, and present these on MHC class II molecules to helper $\mathrm{T}$ cells. Upon recognition helper $\mathrm{T}$ cells will produce cytokines that facilitate B cell proliferation and differentiation into plasma cells or memory B cells. (6) Plasma cells produce antigen-specific antibodies that can neutralize or opsonize pathogens. Opsonized pathogens can be bound by Fc receptors on innate immune cells, resulting in the uptake and destruction of the pathogens. Furthermore, antibodies can bind to antigens exposed on the surface of infected host cells and stimulate antibody-dependent cellular cytotoxicity (ADCC) by innate immune cells. The icons used in this figure are adaptation from icons retrieved from the Servier Medical Art collection, which are licensed under a Creative Commons Attribution 3.0 Unported License (https://creativecommons.org/licenses/by/3.0/).

After the innate immune response is initiated by a vaccine, antigen-presenting cells will activate the adaptive immune system (Figure 2; step 3-5). An important aspect of the adaptive immune response for vaccination is the differentiation of $B$ and $T$ cells into effector and memory cells, of which the latter will quickly induce a secondary immune response upon 
Table I. Myeloid cell lines that have been described for use in potency testing of human and veterinary vaccines.

\begin{tabular}{|c|c|c|c|c|c|}
\hline Name & $\begin{array}{l}\text { Representative } \\
\text { cell type }\end{array}$ & Responds to & $\begin{array}{l}\text { Activation } \\
\text { markers }\end{array}$ & $\begin{array}{l}\text { Does not } \\
\text { respond to }\end{array}$ & Ref. \\
\hline \multirow[t]{3}{*}{ MUTZ-3 } & \multirow[t]{3}{*}{$\begin{array}{l}\text { human dendritic } \\
\text { cells }\end{array}$} & $\begin{array}{l}\text { N. meningitidis outer } \\
\text { membrane protein }\end{array}$ & $\begin{array}{l}\text { CD80, IL6, IL-8, } \\
\text { TNF }\end{array}$ & $\begin{array}{l}\text { LPS, H. influenzae } \\
\text { type B polyribosyl } \\
\text { ribitol phosphate }\end{array}$ & 76 \\
\hline & & & & $\begin{array}{l}\text { Whole-cell } B \text {. } \\
\text { pertussis vaccine }\end{array}$ & 37 \\
\hline & & & & $\begin{array}{l}\text { LPS, R848, whole } \\
\text { inactivated / }\end{array}$ & 75 \\
\hline \multirow[t]{4}{*}{ MM6 } & \multirow[t]{4}{*}{ human monocyte } & LPS, Pam3CSK & $\begin{array}{l}\text { CD80, CD86, } \\
\text { IL- I } \beta, \text { IL-6, IL- } \\
\text { I 2p40, IL- I0 }\end{array}$ & $\begin{array}{l}\text { subunit influenza } \\
\text { virus }\end{array}$ & 37 \\
\hline & & $\begin{array}{l}\text { whole-cell } B \text {. } \\
\text { pertussis vaccine }\end{array}$ & $\begin{array}{l}\text { IL- I } \beta, \text { IL-6, IL- } \\
12 p 40\end{array}$ & & $\begin{array}{l}37 \\
78\end{array}$ \\
\hline & & $\begin{array}{l}\text { N. meningitidis outer } \\
\text { membrane protein }\end{array}$ & $\begin{array}{l}\text { IL- I } \beta, \text { IL-6, IL- } \\
\text { I0, CXCLI0 }\end{array}$ & & 80 \\
\hline & & $\begin{array}{l}\text { LPS, FSL-I, } \\
\text { Pam3CSK4, flagellin, } \\
\text { R848 }\end{array}$ & $\begin{array}{l}\text { IL-I } \beta, \text { IL-6, IL-8, } \\
\text { PGE2,TNF }\end{array}$ & & 81 \\
\hline \multirow[t]{2}{*}{ THP-I } & \multirow[t]{2}{*}{ human monocyte } & PMA+alum & IL-I $\beta$, CD80 & Alum, MF59 & 82 \\
\hline & & MDP+MPLA+alum & IL-I $\beta$, IL-8,TNF & & 83 \\
\hline \multirow[t]{2}{*}{ HDII } & \multirow[t]{2}{*}{$\begin{array}{l}\text { chicken } \\
\text { macrophage }\end{array}$} & $\begin{array}{l}\text { Inactivated vaccines } \\
\text { for infectious } \\
\text { bronchitis virus, } \\
\text { Newcastle disease } \\
\text { virus }\end{array}$ & Phagocytosis & & 84 \\
\hline & & $\begin{array}{l}\text { Inactivated } \\
\text { vaccine for Av. } \\
\text { paragallinarum }\end{array}$ & $\begin{array}{l}\text { Nitric oxide, } \\
\text { IL-I } \beta, \text { CXCLil, } \\
\text { CXCLi2, IL- I0 }\end{array}$ & & 85 \\
\hline
\end{tabular}

future encounter with the same pathogen. Batches of whole cell pertussis vaccines [89] and rabies vaccines [90] of different potencies were found to differ in their ability to induce $T$ cell responses in splenocytes from vaccinated mice, suggesting that $T$ cell assays can be used to test for vaccine potency when these $T$ cell responses are known correlates-of-protection. The activation of naive $T$ cells or the re-activation of memory $T$ cells by vaccines has been mimicked in vitro using respectively autologous dendritic cell-T cell co-cultures or PBMCs to evaluate the potency of vaccines against yellow fever [91] and influenza [92] for use in humans, and for vaccines against blue tongue and rabies for use in cattle [93]. However, $T$ cells are highly heterogenous and $T$ cell assays have only been explored for use in preclinical development of vaccines. In addition, $T$ cell assays with primary cells require blood collection from vaccinated animals, similar to serological assays. The use of a combination of several epitope-specific T hybridoma cell lines might be more promising for potency testing of vaccine batches [94], but requires further development. 
The contribution of assays with innate immune cells to batch testing is product-specific and depends on the presence of PAMPs, adjuvants and other potentially immunostimulatory vaccine components. $T$ cell assays may be useful when $T$ cell responses are known correlates-of-protection. Due to their accessibility, infinitive lifespan, high numbers and low variability, (reporter) cell lines are particularly suitable for cell-based assays to test vaccines. Furthermore, cell-based assays can be useful to investigate whether there are any functional synergistic or antagonistic interactions between the adjuvant and other components in the vaccine, as will be discussed further below. Finally, the need for cell-based assays depends on whether the vaccine will be applied for priming or boosting the immune response. For example, booster vaccines may stimulate the reactivation of memory $T$ cells independently of dendritic cells through memory B cells [93] and are therefore less dependent on stimulating innate immune cells than primer vaccines.

The contribution of assays with innate immune cells to batch testing is product-specific and depends on the presence of PAMPs, adjuvants and other potentially immunostimulatory vaccine components. $T$ cell assays may be useful when $T$ cell responses are known correlates-of-protection. Due to their accessibility, infinite lifespan, high numbers and low variability, (reporter) cell lines are particularly suitable for cell-based assays to test vaccines. Furthermore, cell-based assays can be useful to investigate whether there are any functional synergistic or antagonistic interactions between the adjuvant and other components in the vaccine, as will be discussed further below. Finally, the need for cell-based assays depends on whether the vaccine will be applied for priming or boosting the immune response. For example, booster vaccines may stimulate the reactivation of memory $T$ cells independently of dendritic cells through memory B cells [95] and are therefore less dependent on stimulating innate immune cells than primer vaccines.

\section{Adjuvanted vaccines}

Many inactivated, toxoid and subunit vaccines require adjuvants to support activation of the immune system. For a long period of time, only aluminum salts and mineral oil emulsions were used as adjuvants. Aluminium salts are used as adjuvants for human vaccines such as diphtheria and tetanus vaccines [96] as well as veterinary vaccines such as the bluetongue vaccine for ruminants, some feline leukemia vaccines and the rabies vaccine for dogs, cats, ruminants and horses [97]. The first emulsion adjuvants were Freund's incomplete adjuvant, based on mineral oil emulsified in a water-in-oil formulation, and Freund's complete adjuvant, formulated with tubercle bacteria [98]. Mineral oil-based water-in-oil adjuvants are too reactogenic for use in humans $[99,100]$, but are widely used in poultry, cattle and fish. The first emulsion adjuvant to be approved in humans was MF59, which is an oil-in-water adjuvant based on squalene [IOI].

Before batch release, adjuvanted vaccines are tested for identity, concentration, physical properties like viscosity and stability of the adjuvant (monographs 0062 Vaccines for Veterinary Use and 0153 Vaccines for Human Use; [17]). However, emulsion and aluminum salt adjuvants may interfere with in vitro methods to evaluate the antigens of final products [102]. The following section describes how this problem can be circumvented by testing intermediary products before blending of antigens and adjuvants [103], by extracting the antigenic fraction from final products or by using in vitro methods that are not affected by adjuvants. 
Methods to extract antigens from final adjuvanted products have been developed for several vaccines. To enable the quantification of antigen by ELISAs, isopropyl myristate has been used to extract antigens from oil-adjuvanted poultry vaccines for Newcastle disease, infectious bronchitis and infectious bursal disease [27,104]. Similarly, antigens extracted from an oil-adjuvanted poultry vaccine against infectious coryza could be used to evaluate the presence of PAMPs by measuring their effects on nitric oxide production or cytokine expression in cell-based assays [85]. Isopropyl myristate could not be used to extract antigens from vaccines for food-and-mouth disease, which are formulated with Montanide ISA20I oil adjuvant, but an extraction with benzyl alcohol could be used instead for this vaccine [105], showing that different emulsion adjuvants require different extraction methods. For aluminum salt-adjuvanted vaccines, electrophoretic [106] or chemical competitive desorption methods [107] can be used to retrieve the antigens, although the latter methods may result in denaturation of antigens and affect their integrity. Such methods have been used by manufacturers for hepatitis $A$ vaccines in order to enable antigen quantification by ELISA, but for competitive reasons the methods were not disclosed in the resulting publications $[108,109]$.

Not all quantification methods require antigen extraction or desorption from adjuvanted vaccines. With respect to emulsion adjuvants, a multiplexed sandwich immunoassay for influenza vaccines against strains with pandemic potential was shown to be compatible with the squalene-based adjuvant MF59 [II0]. An in situ method based on the fluorescent nucleic acid-reactive dye SYBR Green II has been developed to determine the stability of an inactivated vaccine for foot-and-mouth disease and was found compatible with aluminum salt, water-in-oil and oil-in-water adjuvanted vaccines [ III]. However, to the best of our knowledge there are currently no in vitro antigen-specific methods to determine the quality of adjuvanted vaccines formulated as water-in-oil emulsions that do not require extraction of the antigen. Methods that non-specifically determine protein content are compatible with aluminum salt adjuvants, including fluorescence spectroscopy, based on intrinsic fluorescence of tyrosine and tryptophan amino acids [1 I2] or fluorescent protein-reactive chemicals [II3], and chemiluminescent nitrogen detection [1 I4]. These methods are not antigen-specific and therefore not suitable for vaccines that contain antigens in complex media with other proteins, e.g. allantoic fluid present in poultry vaccines $[84,1 / 5]$. Some antigen-specific immunoassays have shown compatibility with aluminum salt-adjuvanted vaccines, which include sandwich [1 I6] and competitive [1I7] ELISAs, Luminex [35], and immunofluorescent assays analyzed with a fluorometer [I I8, II9] or flow cytometer [120]. Cell-based toxicity assays can be made compatible for aluminum salt-adjuvanted vaccines by using semi-permeable transwell inserts to prevent direct contact between the cells and aluminum salts, as demonstrated by the $\mathrm{CHO}$ cell-clustering assay used to test for residual toxicity of pertussis vaccines [44].

In summary, adjuvanted vaccines create additional challenges for the development of in vitro potency and safety tests, but adsorption and extraction methods, and the development of adjuvant-compatible methods offer possibilities to overcome these. 


\section{Altered batches to validate in vitro test methods}

Non-compliant vaccine batches of substandard quality are necessary for the validation of new in vitro tests methods by confirming their capacity to discriminate between batches of different qualities [I2I]. However, modern well-controlled vaccine production processes that adhere to GMP guidelines hardly result in failed batches anymore $[11,15]$ and there may be reluctance to share the remaining non-compliant batches. This has led to a paradox in which improved quality of vaccines makes it more difficult to validate new in vitro test methods. Another problem is the inherent variability of in vivo release tests used as gold standards [7-12]. These in vivo tests may show variation to an extent that some batches fail the test, while they are in fact compliant, i.e. their capacity to induce protective immunity in the target species is sufficient. Such batches can be expected to pass appropriate in vitro tests. Likewise, batches may pass in vivo tests, while they are in fact non-compliant, i.e. their capacity to induce protective immunity in the target species is insufficient.

To solve these problems, vaccine batches could be altered intentionally, for example by exposure to stresses that may decrease the stability of a batch during the vaccine production process (e.g. decreased or increased $\mathrm{pH}$, osmolality, temperature) $[1 \mathrm{I}, 3 \mathrm{I}, 12 \mathrm{I}, 122]$, by creating vaccines with reduced antigen content [I I, 122] or a different composition [38], by deviating from the standard inactivation method $[5 \mathrm{I}, 122,123]$, or by changing the degree of adsorption to adjuvants. Importantly, altered batches should be representative for noncompliant batches that may realistically be produced as a result of disturbances in vaccine production processes. In the European Pharmacopoeia, heat treatment is given as an example to create a proper altered batch of the inactivated poliomyelitis vaccines, which is used for product-specific validation of the in vitro D-antigen assay, in order to replace the former in vivo potency test in chickens, guinea pigs or rats (Ph. Eur method 2.7.20, [17]). The altered batches need to be designed and produced already during the development phase of in vitro test methods to identify the sensitivity of these assays for detecting non-compliant batches.

\section{Cooperation between academia, industry and regulatory institutions is crucial for the transition from in vivo to in vitro batch testing of vaccines}

Two previously identified constraints for the transition to in vitro batch testing of vaccines were the lack of priority of research into in vitro test methods that are most needed, and the varying knowledge about vaccine production processes among scientists of different disciplines [15]. International, multi-stakeholder (academia, industry and regulators) collaborations were described as key to move the transition to in vitro vaccine testing forward. Over the last five years, this necessity was met by the VAC2VAC consortium (http://www.vac2vac.eu/), funded by the Innovative Medicines Initiative (IMI2) programme. In this consortium, vaccines and their intermediary products (e.g. antigens, adjuvants, excipients and additives), knowledge about production processes, and new technologies for vaccine quality control were shared between the different stakeholders. The aim of the consortium was to develop new tests and approaches that support the use of the consistency approach in batch testing of established vaccines. 
Assay development is only the first step of the transition from in vivo to in vitro batch testing, which has to be complemented by a validation process [124], first in small-scale feasibility studies to test the transferability of the assay to other labs, later in large-scale multi-center validation studies that are preferably conducted at an international or even global level to standardize novel methods [18]. The latter validation studies can be performed as part of the Biological Standardisation Programme in Europe, in which Official Medicines Control Laboratories (OMCLs), manufacturers and other stakeholders can participate. Assays that have been successfully validated and are accepted by regulatory authorities can become incorporated into pharmacopoeias [124]. After incorporation, the methods still need to be implemented by individual manufacturers and OMCLs, which have to conduct assay validation before the conventional animal-based test can be fully replaced and eventually eliminated from pharmacopoeias. Engagement between academia, industry and regulators at an early stage of the development of in vitro alternatives is essential for getting all stakeholders acquainted with new methods, to facilitate validation studies and to achieve wide implementation of the in vitro alternatives [15,18,72].

\section{Conclusions}

This review describes scientific barriers that hampered the transition from in vivo to in vitro batch testing of vaccines, especially for those that include inactivated pathogens, toxoids or pathogen subunits, and the opportunities to overcome these. An increasing number of in vitro potency and safety tests have been developed to replace in vivo tests. Currently implemented in vitro potency tests largely comprise ELISAs or other immunochemical methods that assess antigen identity, quantity and integrity characteristics, while other properties may also be critical for vaccine quality. There is an increasing consensus that one-to-one replacement by in vitro alternatives is not always possible and that a combination of in vitro alternatives may be needed. Cell-based assays can provide functional information about the interaction between a vaccine and the immune system, which is impossible to capture with ELISAs only. Adjuvanted vaccines are notably hard to test for quality using in vitro alternatives due to their incompatibility with some of these methods. However, the current scientific literature provides many possibilities to overcome these difficulties, including desorption and extraction methods of adjuvants and strategies to study the potential interactions between different vaccine antigens. The use of purposely altered batches enables the validation of new in vitro test methods when there is a lack of non-compliant batches originating from disturbances in the routine vaccine production process due to quality systems and GMP that are in place at the manufacturer. Finally, it is recognized that cooperation between scientists in academia, industry and regulatory institutes is key to move the transition to in vitro vaccine testing forward by sharing of products, knowledge and technologies.

\section{Acknowledgements}

The authors thank prof. dr. Anke Huckriede for providing her insights on the topics addressed in this review. 


\section{Funding}

RJV was supported by the Dutch Ministry of Agriculture, Nature and Food Quality, and the National Institute of Public Health \& the Environment. AS and CFMH were supported by the Dutch Ministry of Agriculture, Nature and Food Quality, and Intravacc.

\section{Supplementary Materials}

Supplementary Table SI can be provided by the authors on request.

\section{References}

I. De Gregorio E, Rappuoli R. From empiricism to rational design: A personal perspective of the evolution of vaccine development. Nat Rev Immunol (2014) I4(7):505-5I4. doi:10.1038/nri3694

2. Milstien J, Dellepiane N, Lambert S, Belgharbi L, Rolls C, Knezevic I, et al. Vaccine quality —can a single standard be defined? Vaccine (2002) 20(7): 1000- I003. doi: I 0.10I 6/S0264-4 I0X(0 I)00422-4

3. Commission to the European Parliament and the Council. 2019 Report on the Statistics on the Use of Animals for Scientific Purposes in the Member States of the European Union in 20152017.; 2020.

4. Hendriksen C, Spieser JM, Akkermans A, Balls M, Bruckner L, Cussler K, et al. Validation of alternative methods for the potency testing of vaccines. Altern Lab Anim (1998) 26(6):747-76I.

5. Milne C, Buchheit KH. EDQM's $3 R$ activities in the field of quality control of vaccines. ALTEX (20I2) I/I2.

6. Walker A, Srinivas GB. Opportunities and strategies to further reduce animal use for Leptospira vaccine potency testing. Biologicals (2013) 4I(5):332-337. doi: I0.1016/j.biologicals.2013.06.006

7. Schiffelers MJ, Blaauboer B, Bakker W, Hendriksen C. Replacing the NIH test for rabies vaccine potency testing: A synopsis of drivers and barriers. Biologicals (2014) 42(4):205-2 I7. doi: 10.10 I6/j. biologicals.20I4.04.00I

8. Servat A, kempff S, Labadie A, Schereffer J-L, Boué F, Cliquet F. In vivo potency tests of rabies inactivated vaccines for veterinary use. A 2-year retrospective analysis of data according to the criteria of the European Pharmacopoeia. Pharmeur Bio Sci Notes (2008) 20:655-664.

9. Goris N, Merkelbach-Peters P, Diev VI, Verloo D, Zakharov VM, Kraft HP, et al. European Pharmacopoeia foot-and-mouth disease vaccine potency testing in cattle: Between test variability and its consequences. Vaccine (2007) 25(I7):3373-3379. doi:10.1016/j.vaccine.2006.12.049

10. Reed NE, Varney WC, Goddard RD, Wyeth PJ. The maintenance of challenge strains used in the potency test for canine leptospira vaccines. Biologicals (2000) 28(I):25-28. doi:10.1006/ biol. 1999.0235

II. EDQM. General chapter 5.2.14 Substitution of in vivo methods by in vitro methods for the quality control of vaccines. In: European Pharmacopoeia. 10th ed. Strasbourgh, France: European Department for the Quality of Medicines; 2019.

12. Xing D, Das RG, O'Neill T, Corbel M, Dellepiane N, Milstien J. Laboratory testing of whole cell pertussis vaccine: a WHO proficiency study using the Kendrick test. Vaccine (200I) 20(3):34235I. doi: I0.1016/S0264-4I0X(0I)00372-3

13. Hendriksen C, Arciniega JL, Bruckner L, Chevalier M, Coppens E, Descamps J, et al. The consistency approach for the quality control of vaccines. Biologicals (2008) 36(I):73-77. doi: 10.1016/j.biologicals.2007.05.002

14. De Mattia F, Hendriksen C, Buchheit KH, Chapsal JM, Halder M, Lambrigts D, et al. T h e vaccines consistency approach project: an EPAA initiative. Pharmeur Bio Sci Notes (2015) 20I5:30-56.

I5. Bruysters MWP, Schiffelers MJ, Hoonakker M, Jungbaeck C, Ragan I, Rommel E, et al. Drivers and 
barriers in the consistency approach for vaccine batch release testing: Report of an international workshop. In: Biologicals. Vol 48. Academic Press; 20 I7:I-5. doi: I0. I0I6/j.biologicals.2017.06.006

16. The European Parliament and the Council of the European Union. DIRECTIVE 2010/63/EU on the protection of animals used for scientific purposes. Off J Eur Union (2010) 276:33-79.

17. EDQM. European Pharmacopoeia. 10.5th. Strasbourgh, France: European Department for the Quality of Medicines; 2020.

18. Halder M, Depraetere H, Delannois F, Akkermans A, Behr-Gross ME, Bruysters M, et al. Recommendations of the VAC2VAC workshop on the design of multi-centre validation studies. In: Biologicals. Vol 52. Academic Press; 2018:78-82. doi:10.1016/j.biologicals.2018.01.003

19. Shank-Retzlaff M, Wang F, Morley T, Anderson C, Hamm M, Brown M, et al. Correlation between mouse potency and in vitro relative potency for human papillomavirus Type 16 virus-like particles and Gardasil vaccine samples. Hum Vaccin (2005) I(5): I9I-197. doi:10.416I/hv. I.5.2126

20. Descamps J, Giffroy D, Remy E, Mortiaux F, Mareschal JC, Ponsar C, et al. A case study of development, validation, and acceptance of a non-animal method for assessing human vaccine potency. Procedia Vaccinol (20II) 5:184-191. doi:10.1016/j.provac.20II.10.018

21. Rosenbruch M. [The sensitivity of chicken embryos in incubated eggs]. ALTEX (1997) I4(3):I I III 3.

22. Settembre EC, Dormitzer PR, Rappuoli R. Bringing influenza vaccines into the 2 I st century. Hum Vaccin Immunother (20I4) I0(3):600-604. doi:10.4I6I/hv.27600

23. Maas RA, Komen M, Van Diepen M, Oei HL, Claassen IJTM. Correlation of haemagglutininneuraminidase and fusion protein content with protective antibody response after immunisation with inactivated Newcastle disease vaccines. Vaccine (2003) 2I(23):3137-3/42. doi:10.1016/ S0264-4I0X(03)00249-4

24. Pay TWF, Hingley PJ. Correlation of I40S antigen dose with the serum neutralizing antibody response and the level of protection induced in cattle by foot-and-mouth disease vaccines. Vaccine (1987) 5(I):60-64. doi:10.1016/0264-4I0X(87)900II-9

25. Poston R, Hill R, Allen C, Casey W, Gatewood D, Levis R, et al. Achieving scientific and regulatory success in implementing non-animal approaches to human and veterinary rabies vaccine testing: A NICEATM and IABS workshop report. In: Biologicals. Vol 60. Academic Press; 2019:8-14. doi:10.1016/j.biologicals.2019.06.005

26. Poirier B, Morgeaux S, Variot $P$, Fuchs F. In vitro potency assay for hepatitis A vaccines: development of a unique economical test. Biologicals (2000) 28(4):247-256. doi:I0.1006/biol.200I.0264

27. Maas PA, de Winter MPM, Venema S, Oei HL, Claassen IJTM. Antigen quantification as in vitro alternative for potency testing of inactivated viral poultry vaccines. Vet Q 2000. doi:10.1080/0 I 652176.2000 .9695063

28. Romstad AB, Reitan LJ, Midtlyng P, Gravningen K, Evensen $\varnothing$. Antibody responses correlate with antigen dose and in vivo protection for oil-adjuvanted, experimental furunculosis (Aeromonas salmonicida subsp. salmonicida) vaccines in Atlantic salmon (Salmo salar L.) and can be used for batch potency testing of vaccin. Vaccine (2013) 3I(5):79I-796. doi: I0.10 I6/j.vaccine.20 I 2.I I.069

29. Coombes L, Tierney R, Rigsby P, Sesardic D, Stickings P. In vitro antigen ELISA for quality control of tetanus vaccines. Biologicals (20I2) 40(6):466-472. doi: I0. I0 I6/j.biologicals.20 I 2.07.0I I

30. Riches-Duit R, Hassall L, Rigsby P, Stickings P. Evaluation of a capture antigen ELISA for the characterisation of tetanus vaccines for veterinary use. Biologicals (2019) 61:8-14. doi:10.1016/j. biologicals.2019.08.003

3I. Coombes L, Stickings P, Tierney R, Rigsby P, Sesardic D. Development and use of a novel in vitro assay for testing of diphtheria toxoid in combination vaccines. J Immunol Methods (2009) 350(I2): I 42-I49. doi:10.1016/j.jim.2009.09.002

32. Westdijk J, Metz B, Spruit N, Tilstra W, van der Gun J, Hendriksen C, et al. Antigenic fingerprinting of diphtheria toxoid adsorbed to aluminium phosphate. Biologicals (2017) 47:69-75. doi:10.1016/j. biologicals.2016.10.005

33. Minor PD. Assaying the potency of influenza vaccines. Vaccines (20I5) 3(I):90-104. doi:10.3390/ vaccines3010090 
34. Westdijk J, Van den ljssel J, Thalen M, Beuvery C, Jiskoot W. Quantification of cell-associated and free antigens in Bordetella pertussis suspensions by antigen binding ELISA. J Immunoassay (1997) I8(3):267-284. doi: 10.1080/0| 97|5297080058।9

35. Agnolon V, Bruno C, Galletti B, Mori E, Ugozzoli M, Pergola C, et al. Multiplex immunoassay for in vitro characterization of acellular pertussis antigens in combination vaccines. Vaccine (2016) 34(8): 1040-1046. doi:10.1016/j.vaccine.2016.01.012

36. Hoonakker ME, Verhagen LM, Pupo E, De Haan A, Metz B, Hendriksen CFM, et al. Vaccinemediated activation of human TLR4 is affected by modulation of culture conditions during wholecell pertussis vaccine preparation. PLoS One (2016) I I (8). doi:I0.137//journal.pone.016I428

37. Hoonakker ME, Verhagen LM, Hendriksen CFM, van Els CACM, Vandebriel RJ, Sloots A, et al. In vitro innate immune cell based models to assess whole cell Bordetella pertussis vaccine quality: $A$ proof of principle. Biologicals (2015) 43(2): 100-109. doi: 10.1016/j.biologicals.20I4.12.002

38. Metz B, Hoonakker M, Uittenbogaard JP, Weyts M, Mommen GPM, Meiring HD, et al.

Proteome analysis is a valuable tool to monitor antigen expression during upstream processing of whole-cell pertussis vaccines. J Proteome Res (2017) 16(2):528-537. doi:10.1021/acs. jproteome.6b00668

39. Maloney T, Phelan R, Simmons N. Saving the horseshoe crab: A synthetic alternative to horseshoe crab blood for endotoxin detection. 2018. doi:I0.137I/journal.pbio.2006607

40. Smith DR, Beekey MA, Brockmann HJ, King TL, Millard MJ, Zaldívar-Rae JA. Limulus polyphemus. The IUCN Red List of Threatened Species. doi:I0.2305/IUCN.UK.2016-I.RLTS. TII987A80I59830.en

4I. Etna MP, Giacomini E, Rizzo F, Severa M, Ricci D, Shaid S, et al. Optimization of the monocyteactivation-test for evaluating pyrogenicity of tick-borne encephalitis virus vaccine. ALTEX May 2020. doi: I0.14573/altex.2002252

42. Hasiwa N, Daneshian M, Bruegger P, Fennrich S, Hochadel A, Hoffmann S, et al. T4 Report evidence for the detection of non-endotoxin pyrogens by the whole blood monocyte activation test. ALTEX (2013) 30(2):169-208. doi:I0.14573/altex.2013.2.169

43. Studholme L, Sutherland J, Desai T, Hockley J, Care R, Nordgren IK, et al. Evaluation of the monocyte activation test for the safety testing of meningococcal $B$ vaccine Bexsero: A collaborative study. Vaccine (2019) 37(29):376I-3769. doi:10.1016/j.vaccine.2018.05.073

44. Isbrucker R, Daas A, Wagner L, Costanzo A. Transferability study of $\mathrm{CHO}$ cell clustering assays for monitoring of pertussis toxin activity in acellular pertussis vaccines. Pharmeur Bio Sci Notes (2016) 20I5:97-II4.

45. Daas A, Behr-Gross ME, Bruckner L, Redhead K. Collaborative study for the validation of cell line assays for in-process toxicity and antigenicity testing of Clostridium septicum vaccine antigens Part I. Pharmeur Bio Sci Notes (2020) 2020:53-124.

46. Behrensdorf-Nicol HA, Weisser K, Krämer B. "BINACLE" assay for in vitro detection of active tetanus neurotoxin in toxoids. ALTEX (20I5) 32(2): I37-I42. doi:I0.14573/altex.14I2I8I

47. Committee for Human Medicinal Products - European Medicines Agency. ICH guideline Q8 (R2) on pharmaceutical development. 2017:I-24. doi:10.1002/bit.22326

48. Clough NEC, Hauer PJ. Using polyclonal and monoclonal antibodies in regulatory testing of biological products. ILAR J (2005) 46(3):300-306. doi: I0.1093/ilar.46.3.300

49. Geeraedts F, ter Veer W, Wilschut J, Huckriede A. Effect of viral membrane fusion activity on antibody induction by influenza $\mathrm{H} 5 \mathrm{NI}$ whole inactivated virus vaccine. Vaccine (2012) 30(45):650I-6507. doi:10.1016/j.vaccine.2012.07.036

50. Jagt HJM, Bekkers MLE, van Bommel SAJT, van der Marel P, Schrier CC. The influence of the inactivating agent on the antigen content of inactivated Newcastle disease vaccines assessed by the in vitro potency test. Biologicals (2010) 38(I):I28-I34. doi:I0.10 I6/j.biologicals.2009.07.006

5I. Metz B, Tilstra W, van der Put R, Spruit N, van den IJssel J, Robert J, et al. Physicochemical and immunochemical assays for monitoring consistent production of tetanus toxoid. Biologicals (20I3) 4 I (4):23 I-237. doi: 10.10 I6/j.biologicals.20I3.05.00 I

52. van de Waterbeemd B, Streefland M, Pennings J, van der Pol L, Beuvery C, Tramper J, et al. Gene- 
expression-based quality scores indicate optimal harvest point in Bordetella pertussis cultivation for vaccine production. Biotechnol Bioeng (2009) 103(5):900-908. doi:10.1002/bit.22326

53. Egger $M$, Jürets $A$, Wallner $M$, Briza $P$, Ruzek $S$, Hainzl $S$, et al. Assessing protein immunogenicity with a dendritic cell line-derived endolysosomal degradome. PLoS One (20II) 6(2):el7278. doi:10.1371/journal.pone.0017278

54. Michiels TJM, Meiring HD, Jiskoot W, Kersten GFA, Metz B. Formaldehyde treatment of proteins enhances proteolytic degradation by the endo-lysosomal protease cathepsin S. Sci Rep (2020) I0(I): I I535. doi: I0.1038/s4I598-020-68248-z

55. Michiels TJM, Tilstra W, Hamzink MRJ, de Ridder JW, Danial M, Meiring HD, et al. Degradomicsbased analysis of tetanus toxoids as a quality control assay. Vaccines (2020) 8(4):7I 2. doi: I 0.3390/ vaccines80407/2

56. Hagenaars N, Mastrobattista E, Glansbeek H, Heldens J, van den Bosch H, Schijns V, et al. Headto-head comparison of four nonadjuvanted inactivated cell culture-derived influenza vaccines: Effect of composition, spatial organization and immunization route on the immunogenicity in a murine challenge model. Vaccine (2008) 26(5I):6555-6563. doi: 10.1016/j.vaccine.2008.09.057

57. Geeraedts F, Goutagny N, Hornung V, Severa M, de Haan A, Pool J, et al. Superior immunogenicity of inactivated whole virus $\mathrm{H} 5 \mathrm{NI}$ influenza vaccine is primarily controlled by toll-like receptor signalling. Subbarao K, ed. PLoS Pathog (2008) 4(8):el000 I38. doi:I0.137//journal.ppat. I000I 38

58. Bernstein DI, Zahradnik JM, DeAngelis CJ, Cherry JD. Clinical reactions and serologic responses after vaccination with whole-virus or split-virus influenza vaccines in children aged 6 to 36 months. Pediatrics (1982) 69(4).

59. Halbroth BR, Heil A, Distler E, Dass M, Wagner EM, Plachter B, et al. Superior in vitro stimulation of human CD8+ T-cells by whole virus versus split virus influenza vaccines. Stambas J, ed. PLoS One (2014) 9(7):el03392. doi:10.1371/journal.pone.0103392

60. Hampson AW. Vaccines for pandemic influenza. The history of our current vaccines, their limitations and the requirements to deal with a pandemic threat. Ann Acad Med Singapore (2008) 37(6):510-517.

61. Bachmann MF, Jennings GT. Vaccine delivery: A matter of size, geometry, kinetics and molecular patterns. Nat Rev Immunol (20I0) I0(I I):787-796. doi:I0.1038/nri2868

62. Reynolds DL, Maraqa AD. Protective immunity against Newcastle disease: The role of cellmediated immunity. Avian Dis (2000) 44(I): I45-I54. doi:10.2307/I5925 I8

63. Higgins SC, Jarnicki AG, Lavelle EC, Mills KHG. TLR4 mediates vaccine-induced protective cellular immunity to Bordetella pertussis : Role of IL- I7-producing T cells. J Immunol (2006) I77(I I):79807989. doi:10.4049/jimmunol.177.1 I.7980

64. Herrera-Rodriguez J, Signorazzi A, Holtrop M, de Vries-Idema J, Huckriede A. Inactivated or damaged? Comparing the effect of inactivation methods on influenza virions to optimize vaccine production. Vaccine (2019) 37(12):1630-1637. doi:10.1016/j.vaccine.2019.01.086

65. Viviani L, Halder M, Gruber M, Bruckner L, Cussler K, Sanyal G, et al. Global harmonization of vaccine testing requirements: Making elimination of the ATT and TABST a concrete global achievement. In: Biologicals. Vol 63. Academic Press; 2020:101-105. doi:10.1016/j. biologicals.2019.10.007

66. Garbe JHO, Ausborn S, Beggs C, Bopst M, Joos A, Kitashova AA, et al. Historical data analyses and scientific knowledge suggest complete removal of the abnormal toxicity test as a quality control test. J Pharm Sci (20 I4) I03(I I):3349-3355. doi:I0. 1002/jps.24I 25

67. Spohr C, Kaufmann E, Battenfeld S, Duchow K, Cussler K, Balks E, et al. A new lymphocyte proliferation assay for potency determination of bovine tuberculin PPDs. ALTEX (2015) 32(3):20I-210. doi:10.14573/altex.150210I

68. Neverov A, Chumakov K. Massively parallel sequencing for monitoring genetic consistency and quality control of live viral vaccines. Proc Natl Acad Sci (2010) 107(46):20063 LP - 20068. doi:10.1073/pnas.1012537107 
69. Charlton B, Hockley J, Laassri M, Wilton T, Crawt L, Preston M, et al. The use of nextgeneration sequencing for the quality control of live-attenuated polio vaccines. J Infect Dis (2020) 222(II):1920-1927. doi:10.1093/infdis/jiaa299

70. da Costa A, Prehaud C, Khou C, Pardigon N, Saulnier A, Nougarede N, et al. Innovative in cellulo method as an alternative to in vivo neurovirulence test for the characterization and quality control of human live Yellow Fever virus vaccines: A pilot study. Biologicals (2018) 53:19-29. doi:10.1016/j.biologicals.2018.03.004

7I. Szymkowicz L, Wilson DJ, James DA. Development of a targeted nanoLC-MS/MS method for quantitation of residual toxins from Bordetella pertussis. J Pharm Biomed Anal (2020) I88:I I 3395. doi:10.1016/j.jpba.2020.113395

72. Akkermans A, Chapsal JM, Coccia EM, Depraetere H, Dierick JF, Duangkhae P, et al. Animal testing for vaccines. Implementing replacement, reduction and refinement: challenges and priorities. Biologicals October 2020. doi:10.1016/j.biologicals.2020.07.010

73. Vandebriel R, Hoefnagel MMN. Dendritic cell-based in vitro assays for vaccine immunogenicity. Hum Vaccin Immunother (2012) 8(9):I323-1325. doi:I0.4I6I/hv.21350

74. Stoel M, Pool J, de Vries-Idema J, Zaaraoui-Boutahar F, Bijl M, Andeweg AC, et al. Innate responses induced by whole inactivated virus or subunit influenza vaccines in cultured dendritic cells correlate with immune responses in vivo. PLoS One (20I5) I0(5):e0I25228. doi:I0.137I/ journal.pone.0125228

75. Tapia-Calle G, Stoel M, de Vries-Idema J, Huckriede A. Distinctive responses in an in vitro human dendritic cell-based system upon stimulation with different influenza vaccine formulations. Vaccines (20I7) 5(3):2I. doi:10.3390/vaccines503002I

76. Hoefnagel MHN, Vermeulen JP, Scheper RJ, Vandebriel RJ. Response of MUTZ-3 dendritic cells to the different components of the Haemophilus influenzae type B conjugate vaccine: Towards an in vitro assay for vaccine immunogenicity. Vaccine (20II) 29(32):5II4-5I2I. doi:I0.10I6/j. vaccine.20II.05.050

77. Querec T, Bennouna S, Alkan S, Laouar Y, Gorden K, Flavell R, et al. Yellow fever vaccine YF-I 7D activates multiple dendritic cell subsets via TLR2, 7, 8, and 9 to stimulate polyvalent immunity. J Exp Med (2006) 203(2):4I3-424. doi:I0.1084/jem.2005I720

78. Brummelman J, Veerman RE, Hamstra HJ, Deuss AJM, Schuijt TJ, Sloots A, et al. Bordetella pertussis naturally occurring isolates with altered lipooligosaccharide structure fail to fully mature human dendritic cells. McCormick BA, ed. Infect Immun (20I5) 83(I):227-238. doi:I0.I I 28/IAI.02 I 97-I4

79. Hovingh ES, van Gent M, Hamstra H-J, Demkes M, Mooi FR, Pinelli E. Emerging Bordetella pertussis strains induce enhanced signaling of human pattern recognition receptors TLR2, NOD2 and secretion of IL-10 by dendritic cells. Hozbor DF, ed. PLoS One (2017) I2(I):e0170027. doi:10.1371/journal.pone.0170027

80. Zariri A, Beskers J, van de Waterbeemd B, Hamstra HJ, Bindels THE, van Riet E, et al. Meningococcal outer membrane vesicle composition-dependent activation of the innate immune response. Palmer GH, ed. Infect Immun (2016) 84(I0):3024 LP - 3033. doi:I 0.I I 28/IAI.00635-I6

8I. Zaitseva M, Romantseva T, Blinova K, Beren J, Sirota L, Drane D, et al. Use of human MonoMac6 cells for development of in vitro assay predictive of adjuvant safety in vivo. Vaccine (2012) 30(32):4859-4865. doi:10.1016/j.vaccine.2012.05.002

82. Vrieling H, Kooijman S, de Ridder JW, Thies-Weesie DME, Soema PC, Jiskoot W, et al. Activation of human monocytes by colloidal aluminum salts. J Pharm Sci (2020) I09(I):750-760. doi: I0.1016/j. xphs.2019.08.014

83. Tukhvatulin Al, Dzharullaeva AS, Tukhvatulina NM, Shcheblyakov D V, Shmarov MM, Dolzhikova I $V$, et al. Powerful complex immunoadjuvant based on synergistic effect of combined TLR4 and NOD2 activation significantly enhances magnitude of humoral and cellular adaptive immune responses. PLoS One (2016) I I (5):e0 I55650. doi:I0. I37 I/journal.pone.0I55650

84. van den Biggelaar RHGA, van Eden W, Rutten VPMG, Jansen CA. Nitric oxide production and Fc receptor-mediated phagocytosis as functional readouts of macrophage activity upon stimulation with inactivated poultry vaccines in vitro. Vaccines (2020) 8(2):332. doi: 10.3390/vaccines8020332 
85. van den Biggelaar RHGA, van Eden W, Rutten VPMG, Jansen CA. Macrophage activation assays to evaluate the immunostimulatory capacity of Avibacterium paragallinarum in a multivalent poultry vaccine. Vaccines (2020) 8(4):67I. doi:I0.3390/vaccines804067I

86. Hoffmann S, Peterbauer A, Schindler S, Fennrich S, Poole S, Mistry Y, et al. International validation of novel pyrogen tests based on human monocytoid cells. J Immunol Methods (2005) 298(I2):16I-173. doi:10.1016/j.jim.2005.01.010

87. Zhu J, Yamane H, Paul WE. Differentiation of effector CD4+ T cell populations. Annu Rev Immunol (20I0) 28:445-489. doi:I0.I I 46/annurev-immunol-030409-I0I 2 I 2

88. Bent R, Moll L, Grabbe S, Bros M. Interleukin-I beta-A friend or foe in malignancies. Int J Mol Sci (2018) 19(8):2155. doi:10.3390/ijms I 9082155

89. Hoonakker ME, Verhagen LM, van der Maas L, Metz B, Uittenbogaard JP, van de Waterbeemd $B$, et al. Adaptive immune response to whole cell pertussis vaccine reflects vaccine quality: $A$ possible complementation to the Pertussis Serological Potency test. Vaccine (2016) 34(37):44294436. doi:10.1016/j.vaccine.2016.07.011

90. Joffret ML, Zanetti C, Morgeaux S, Leclerc C, Sureau P, Perrin P. Appraisal of rabies vaccine potency by determination of in vitro, specific interleukin-2 production. Biologicals (1991) 19(2): I I3-123. doi:10.1016/1045-1056(91)90009-9

91. Moser JM, Sassano ER, Leistritz DC, Eatrides JM, Phogat S, Koff W, et al. Optimization of a dendritic cell-based assay for the in vitro priming of naïve human CD4+ T cells. J Immunol Methods (2010) 353(I-2):8-19. doi:10.1016/J.JIM.2009.1I.006

92. Tapia-Calle G, Born PA, Koutsoumpli G, Gonzalez-Rodriguez MI, Hinrichs WLJ, Huckriede ALW. A PBMC-based system to assess human $T$ cell responses to influenza vaccine candidates in vitro. Vaccines (2019) 7(4): I8I. doi: 10.3390/vaccines7040I8I

93. Kangethe RT, Pichler R, Chuma FNJ, Cattoli G, Wijewardana V. Bovine monocyte derived dendritic cell based assay for measuring vaccine immunogenicity in vitro. Vet Immunol Immunopathol (2018) 197:39-48. doi:10.1016/j.vetimm.2018.01.009

94. Yu SCT, Nag B. Application of murine T-T hybridoma cells to in vitro potency assay of human synthetic peptide vaccines. Vaccine (1996) I4(I4):I313-132I. doi:10.1016/S0264$410 \times(96) 00066-7$

95. Ise W, Inoue T, McLachlan JB, Kometani K, Kubo M, Okada T, et al. Memory B cells contribute to rapid Bcl6 expression by memory follicular helper T cells. Proc Natl Acad Sci U S A (2014) I I I(32): I 1792- I |797. doi: I0.1073/pnas. I 40467| I II

96. Marrack P, McKee AS, Munks MW. Towards an understanding of the adjuvant action of aluminium. Nat Rev Immunol (2009) 9(4):287-293. doi: I0.1038/nri25I0

97. Spickler AR, Roth JA. Adjuvants in veterinary vaccines: modes of action and adverse effects. J Vet Intern Med (2003) I7(3):273-28I. doi:10.1 I I I/j.1939-1676.2003.tb02448.x

98. Freund J, Casals J, Hosmer EP. Sensitization and antibody formation after injection of tubercle bacilli and paraffin oil. Proc Soc Exp Biol Med (1937) 37(3):509-513. doi:10.3181/00379727-379625

99. Di Pasquale A, Preiss S, Da Silva FT, Garçon N. Vaccine adjuvants: From 1920 to 2015 and beyond. Vaccines (20I5) 3(2):320-343. doi: 10.3390/vaccines3020320

100. Van Doorn E, Liu H, Huckriede A, Hak E. Safety and tolerability evaluation of the use of Montanide ISATM5I as vaccine adjuvant: A systematic review. Hum Vaccines Immunother (2016) I2(I):I59169. doi:10.1080/2I6455I5.20I5.107|455

10I. O'Hagan DT, Ott GS, Nest G Van, Rappuoli R, Giudice G Del. The history of MF59 ® adjuvant: a phoenix that arose from the ashes. Expert Rev Vaccines (2013) I2(I):13-30. doi:10.1586/ erv. 12.140

102. Misquith A, Fung HWM, Dowling QM, Guderian JA, Vedvick TS, Fox CB. In vitro evaluation of TLR4 agonist activity: formulation effects. Colloids Surf B Biointerfaces (2014) II3:3 I2-3 I9. doi:10.1016/j.colsurfb.2013.09.006 
103. Stokes W, Srinivas G, McFarland R, Kulpa-Eddy J, Casey W, Walker A, et al. Report on the international workshop on alternative methods for Leptospira vaccine potency testing: State of the science and the way forward. Biologicals (2013) 4I(5):279-294. doi:10.1016/j. biologicals.2013.06.013

104. Claassen I, Maas R, Oei H, Daas A, Milne C. Validation study to evaluate the reproducibility of a candidate in vitro potency assay of newcastle disease vaccines and to establish the suitability of a candidate biological reference preparation. Pharmeuropa bio (2004) 2004(I): I-I5.

105. Saravanan P, Iqbal Z, Selvaraj DPR, Aparna M, Umapathi V, Krishnaswamy N, et al. Comparison of chemical extraction methods for determination of $146 \mathrm{~S}$ content in foot-and-mouth disease oil-adjuvanted vaccine. J Appl Microbiol (2020) I 28(I):65-73. doi: I 0. I I I I/jam. I4465

106. Morgenroth A, Jakel V, Hanke-Robinson H, Müller T, Freuling C, Cussler K, et al. A novel electrophoretic immunoblot as antigen desorption and quantification method for alum-adjuvanted veterinary rabies vaccines. Vaccine (2020) 38(27):428I-4287. doi:I 0.10I6/j.vaccine.2020.04.057

107. Rinella J V., Workman RF, Hermodson MA, White JL, Hem SL. Elutability of proteins from aluminum-containing vaccine adjuvants by treatment with surfactants. J Colloid Interface Sci (1998) 197(I):48-56. doi:10.1006/jcis. 1997.5230

108. Stalder J, Costanzo A, Daas A, Rautmann G, Buchheit K-H. Establishment of a biological reference preparation for hepatitis A vaccine (inactivated, non-adsorbed). Pharmeur Bio Sci Notes (2010) 2010(I):I5-29.

109. Wood D, Sands D, Biol AH-PSI, 2000 U. Collaborative study for the establishment of three product specific European Pharmacopoeia biological reference preparations for inactivated adsorbed hepatitis A vaccines. Pharmeur Bio Sci Notes 2000.

I I0. Byrne-Nash RT, Miller DF, Bueter KM, Gillis JH, Kuck LR, Rowlen KL. VaxArray potency assay for rapid assessment of "pandemic" influenza vaccines. npj Vaccines (20I8) 3(I):43. doi:I0.1038/ s4I54I-0I8-0080-6

III. Song Y, Yang Y, Lin X, Li X, Zhang X, Ma G, et al. In-situ and sensitive stability study of emulsion and aluminum adjuvanted inactivated foot-and-mouth disease virus vaccine by differential scanning fluorimetry analysis. Vaccine (2020) 38(14):2904-29I2. doi:I0.1016/j.vaccine.2020.02.068

1 12. Nouchikian L, Roque C, Song JY, Rahman N, Ausar SF. An intrinsic fluorescence method for the determination of protein concentration in vaccines containing aluminum salt adjuvants. Vaccine (2018) 36(38):5738-5746. doi:10.1016/j.vaccine.2018.08.005

I 13. Zhu D, Saul A, Huang S, Martin LB, Miller LH, Rausch KM. Use of o-phthalaldehyde assay to determine protein contents of Alhydrogel-based vaccines. Vaccine (2009) 27(43):6054-6059. doi:10.1016/j.vaccine.2009.07.067

II4. Amari J V., Levesque P, Lian Z, Lowden T, DeAlwis U. Concentration determination of a recombinant vaccine antigen adsorbed onto an alum adjuvant by chemiluminescent nitrogen detection. Pharm Res (2005) 22(I):33-37. doi: I0.1007/s I 1095-004-9006-2

1 15. Da Silva M, Labas V, Nys Y, Réhault-Godbert S. Investigating proteins and proteases composing amniotic and allantoic fluids during chicken embryonic development. Poult Sci (2017) 96(8):2931294I. doi: I0.3382/ps/pex058

I16. Katz JB, Hanson SK, Patterson PA, Stoll IR. In vitro assessment of viral antigen content in inactivated aluminum hydroxide adjuvanted vaccines. J Virol Methods (1989) 25(1): 10I-108. doi:10.1016/0166-0934(89)90104-3

117. Necchi F, Carducci M, Pisoni I, Rossi O, Saul A, Rondini S. Development of FAcE (Formulated Alhydrogel competitive ELISA) method for direct quantification of OAg present in Shigella sonnei GMMA-based vaccine and its optimization using Design of Experiments approach. J Immunol Methods (2019) 471:11-17. doi:10.1016/j.jim.2019.04.012

I 18. Zhu D, Huang S, Gebregeorgis E, McClellan H, Dai W, Miller L, et al. Development of a direct alhydrogel formulation immunoassay (DAFIA). J Immunol Methods (2009) 344(I):73-78. doi:10.1016/j.jim.2009.03.005

1 19. Li M, Wang X, Cao L, Lin Z, Wei M, Fang M, et al. Quantitative and epitope-specific antigenicity analysis of the human papillomavirus 6 capsid protein in aqueous solution or when adsorbed on 
particulate adjuvants. Vaccine (2016) 34(37):4422-4428. doi:I0.1016/j.vaccine.2016.07.012

120. Ugozzoli M, Laera D, Nuti S, Skibinski DAG, Bufali S, Sammicheli C, et al. Flow cytometry: An alternative method for direct quantification of antigens adsorbed to aluminum hydroxide adjuvant. Anal Biochem (20II) 4I8(2):224-230. doi:10.1016/j.ab.20II.07.012

121. Gibert R, Alberti M, Poirier B, Jallet C, Tordo N, Morgeaux S. A relevant in vitro ELISA test in alternative to the in vivo $\mathrm{NIH}$ test for human rabies vaccine batch release. Vaccine (2013) 3 I (50):6022-6029. doi:I0.10I6/j.vaccine.2013.10.019

122. Chabaud-Riou M, Moreno N, Guinchard F, Nicolai MC, Niogret-Siohan E, Sève N, et al. G-protein based ELISA as a potency test for rabies vaccines. Biologicals (2017) 46:124-129. doi:10.1016/j. biologicals.2017.02.002

123. Toinon A, Moreno N, Chausse H, Mas E, Nicolai MC, Guinchard F, et al. Potency test to discriminate between differentially over-inactivated rabies vaccines: Agreement between the $\mathrm{NIH}$ assay and a G-protein based ELISA. Biologicals (2019) 60:49-54. doi:10.1016/j.biologicals.2019.05.004

124. Schiffelers MJWA, Blaauboer BJ, Bakker WE, Hendriksen CFM. Regulatory acceptance and use of serology for inactivated veterinary rabies vaccines. ALTEX (20I5) 32(3):2 I I-22I. doi: I0.14573/ altex. $|50| 26 \mid$ 


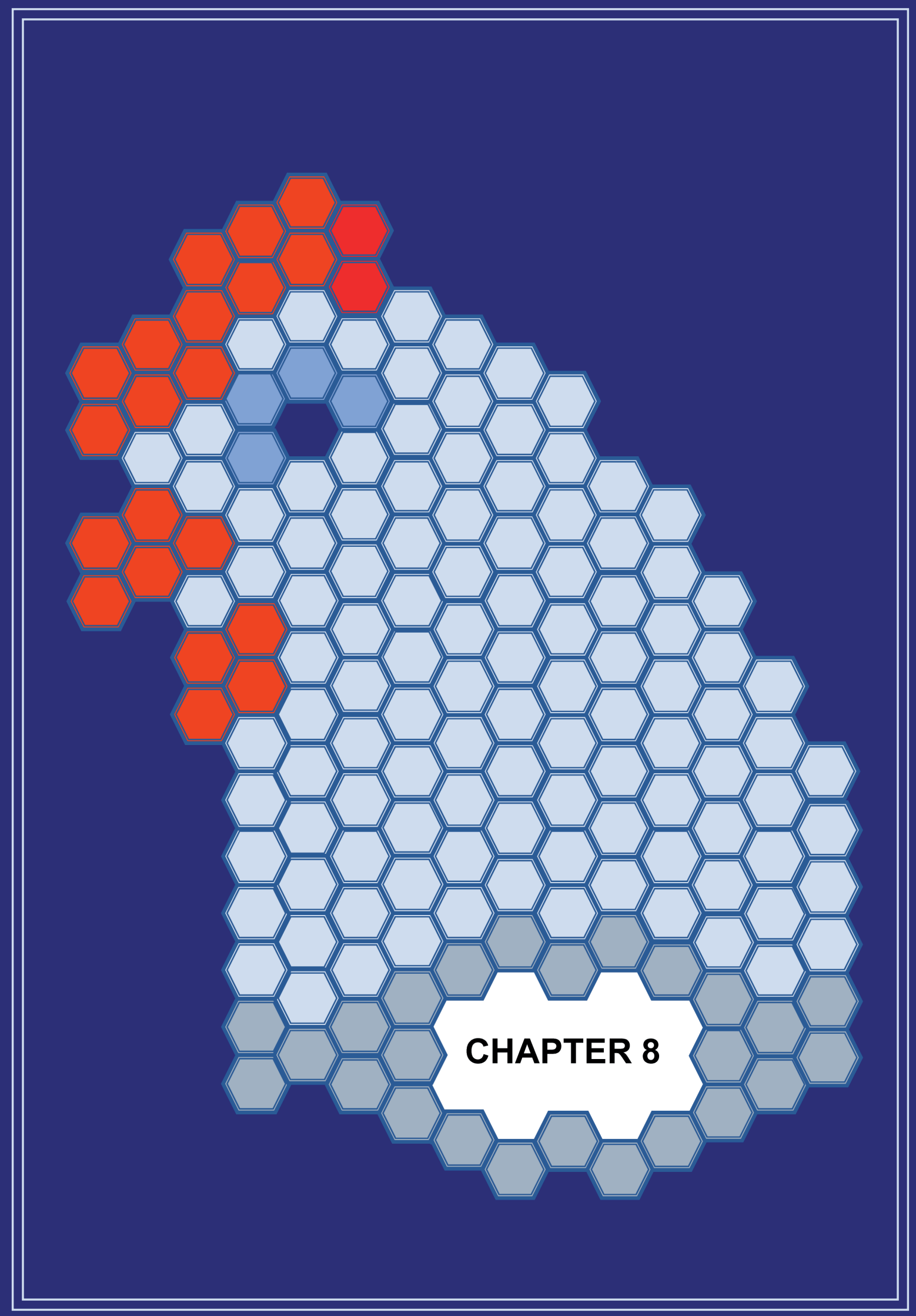


Summarizing discussion 


\section{Summarizing discussion}

More than a million animals are used annually in the European Union for batch release tests of medicinal products, predominantly for potency assessment [I]. A quarter of these tests are classified as causing severe pain and distress. With respect to inactivated poultry vaccines, most potency tests are still performed in vivo due to the lack of in vitro alternatives. It is important to move away from in vivo testing because of ethical concerns [2-5], variability, long duration and high costs of these tests [4,5], risks for personnel working with infected animals [4], and legal requirements to use in vitro methods instead of in vivo methods whenever possible [6]. The primary aim of this thesis was to set up in vitro cell-based assays to assess the immunostimulatory capacity of inactivated poultry vaccines and to determine their applicability for routine potency testing. For this purpose, we have set up novel cellbased assays using a macrophage cell line to assess the immunostimulatory capacity of inactivated poultry vaccines (Chapters 2 and 3). Secondly, we have used bone marrowderived dendritic cells to search for novel biomarkers of immune activation by the vaccines (Chapters 4 and 5). Thirdly, we explored the applicability of a T cell proliferation assay in quality assessment of inactivated poultry vaccines (Chapter 6). Finally, we reviewed the recent progress made for the transition from in vivo to in vitro quality testing of veterinary and human vaccines (Chapter 7).

\section{Cell-based assays to evaluate the capacity of inactivated poultry vaccines to stimulate innate immune cells}

Innate immune cells sense pathogens with pattern recognition receptors The extent to which innate immune cells sense and respond to immunostimulatory molecules of vaccines, including pathogen-associated molecular patterns (PAMPs) and adjuvants, depends on interactions with pattern-recognition receptors (PRRs). The chicken macrophage-like cell line HDII [7] provides a suitable model to investigate innate immune responses to vaccines due to its broad expression of PRRs including e.g. toll-like receptors (TLRs). Our experiments showed that HDII macrophages respond by increased phagocytosis (Chapter 2) and nitric oxide production (Chapter 3), two wellknown effector functions of macrophages, to a range of bacterial, fungal and viral PAMPs or synthetic analogs. This demonstrated that the cell line is capable of detecting a diversity of immunostimulatory molecules potentially present in inactivated poultry vaccines. The only exception was poly(l:C), a synthetic analog of double-stranded RNA and representative for the genetic material of some viruses, to which HDI I cells did not respond [8-I0] (Chapter 3). Other studies have demonstrated that transfection of poly $(\mathrm{l}: \mathrm{C})$ or double-stranded RNA to the cytosol may be required for recognition by HDII cells $[8, \mathrm{II}]$.

Immune responsiveness of the HDI I cell line to inactivated infectious bronchitis virus, Newcastle disease virus and egg-drop syndrome virus vaccines in vitro

The HDI I cell line was stimulated with inactivated vaccines against the single-stranded RNA viruses IBV and NDV, and the double-stranded DNA virus EDSV (Chapters 2 and 3). Recognition of the single stranded RNA viruses occurs through TLR7 [12], while recognition of the double-stranded DNA virus EDSV is expected to occur through TLR2I. 
Previous studies have observed increased nitric oxide production and cytokine expression by HDI I cells in response to virulent NDV recognized, which was sensed through TLR7 [13]. We have also observed functionality of both TLR7 and TLR2I in HDII cells after stimulation with the TLR agonists R848 (i.e. resiquimod) and oligodeoxyribonucleotides with CPG motifs, respectively (Chapters 2 and 3). Despite the presence of functional TLR7 and TLR2I, our results showed that a trivalent vaccine comprising whole-inactivated IBV, NDV and EDSV did not induce nitric oxide production or enhanced cytokine expression (Chapter 3). Thus, there is a clear difference in the response of HDII cells to virulent NDV [13] and the inactivated NDV vaccines used in this thesis. In contrast, the trivalent and other inactivated vaccines comprising IBV and NDV stimulated an increase in Fc receptormediated phagocytosis, which was caused by allantoic fluid present in the vaccine rather than inactivated viruses (Chapter 2).

It is possible that preservation of the virions present in the vaccine and recognition of the viral nucleic acids were affected by inactivation methods [14,15]. Alternatively, the inactivated viruses might not have ended up in the endosomal compartments where TLR7 and TLR2I are present, though vaccine uptake was confirmed microscopically by accumulation of droplets of mineral oil adjuvant (Chapter 2). Finally, although it has been shown that chicken immune cells respond to ligands known to activate mammalian TLR7 (e.g. R848) [12], activation of chicken TLR7 itself by either these ligands or single-stranded RNA through e.g. reporter gene assays has not yet been demonstrated $[16,17]$. Taken together, although the cell-based assays with the HDII cell line showed effects of TLR ligands, we did not observe immune responsiveness associated with inactivated virions in vaccines for IBV, NDV and EDSV. The presented cell-based assays with the HDII cell line may therefore be of limited use in potency testing of inactivated poultry vaccines of viral origin. Inactivated vaccines for IBV and NDV are often used in a prime-boost vaccination scheme and are preceded by live attenuated vaccines. Therefore, the working mechanism of these vaccines may rely predominantly on reactivation of memory $B$ and $T$ cells rather than activation of innate immune cells.

\section{Immune responsiveness of the HDI I cell line to inactivated vaccine against Avibacterium paragallinarum in vitro}

Studies on Avibacterium (Av.) paragallinarum bacteria and their interaction with immune cells are limited, despite the fact that the resulting disease, infectious coryza, leads to high mortality and drops in egg production [18]. The HDII cell line was used to study the immunostimulatory capacity of an octavalent vaccine comprising five different $A v$. paragallinarum strains, as well as the three viruses IBV, NDV and EDSV (Chapter 3). The cell line showed increased nitric oxide production and gene expression of the cytokines IL- $\beta$, CXCLil, CXCLi2, TNF, IL-I0 and IL-I2p40 after stimulation with inactivated $A v$. paragallinarum bacteria. The vaccine-induced responses were significantly reduced when the inactivated bacteria were pre-incubated with the LPS-binding antibiotic polymyxin B [19], indicating that activation occurred at least partially through the LPS-recognizing PRR TLR4. In addition to TLR4, the inactivated Av. paragallinarum vaccine may activate HDII cells through stimulation of other TLRs such as TLRI/2 heterodimers or TLR2I. Specific inhibitors for chicken TLR2I do not yet exist. Using the inhibitor CU-CPT22 of mammalian TLRI/2, we have tried to determine the contribution of chicken TLRI/2 to the activation of 
HDI I cells by Av. paragallinarum. Unfortunately, CU-CPT22 did not inhibit the functionality of chicken TLRI/2, even after stimulation with the chicken TLRI/2 agonist Pam3CSK4 (not shown).

Taken together, our study has demonstrated that immunostimulatory molecules, at least LPS, are present in inactivated Av. paragallinarum vaccines and that their presence can be measured in cell-based assays with HDII cells for potency testing. Future studies are needed to fully reveal whether immunostimulatory molecules other than LPS are present in inactivated $A v$. paragallinarum vaccines and need to be considered in potency testing.

\section{Allantoic fluid}

In order to produce vaccines, IBV, NDV and EDSV are propagated in the allantoic cavity of embryonated chicken eggs and obtained by collecting allantoic fluid. We observed that exposure of HDI I cells to allantoic fluid led to increased expression of chicken high-affinity IgY receptor CHIR-ABI and enhanced Fc receptor-mediated phagocytosis (Chapter 2). Similarly, increased expression of CHIR-ABI on natural killer cells in response to infectious bursal disease virus (IBDV) after propagation on embryonated chicken eggs was reported [20].

Further evidence for the immunostimulatory capacity of allantoic fluid was found when we stimulated chicken bone marrow-derived dendritic cells (chBMDCs) with an IBV antigen preparation resulting in upregulation of spleen tyrosine kinase (SYK) (Chapter 5). This protein is involved in both Fc receptor-mediated phagocytosis and inflammasome activation in response to monosodium urate crystals [2I-23]. These crystals are damageassociated molecular patterns (DAMPs) that are the result of precipitation of uric acid at high concentrations as present in allantoic fluid [24,25]. Activation of inflammasomes by monosodium urate crystals is important for the conversion of pro-interleukin (IL)-I $\beta$, proIL-I 8 and pro-IL-33 into active pro-inflammatory cytokines $[25,26]$.

An immunostimulatory function of allantoic fluid has not been studied before. Based on the presence of monosodium urate crystals, allantoic fluid is able to induce Fc receptormediated phagocytosis through upregulation of CHIR-ABI and is likely to have a role in IL-I $\beta$ secretion through activation of inflammasomes. The contribution of allantoic fluid present in inactivated poultry vaccines to immune activation will be interesting for future studies.

\section{Nitric oxide production by the HDI I cell line to evaluate the potency of inactivated poultry vaccines}

Previous studies have demonstrated that HDI I cells show enhanced nitric oxide production and cytokine expression in the presence of replicating viruses [13,27,28], which we did not observe for poultry vaccines containing inactivated viruses (Chapter $\mathbf{3}$ ). In contrast to the inactivated viruses, inactivated Av. paragallinarum bacteria were found to activate the HDII cell line resulting in nitric oxide production and enhanced cytokine expression. So, the capacity to stimulate innate immune cells in vitro was apparently lost for viral vaccines after inactivation, but remained intact for Av. paragallinarum. 
Inactivated vaccines for Av. paragallinarum are currently tested for potency using in vivo vaccination-challenge studies, which inflict pain and distress to large groups of animals. The use of an alternative serological assay has been explored, but a correlation between vaccine potency and antibody titers was not found [29]. The nitric oxide production assay was fast and easy to perform, and seems a promising in vitro alternative. Using this assay, the potency of inactivated Av. paragallinarum bacteria can expressed as the half-maximum effective concentration $\left(\mathrm{EC}_{50}\right)$, allowing easy comparison between batches. The toxicity of some vaccine constituents (e.g. inactivation agent and preservatives) was overcome by extracting the bacterial contents from the adjuvanted vaccine using isopropyl myristate and methods similar to those used for antigen quantification of inactivated NDV vaccines [30].

Previously it was proposed to use the nitric oxide production assay for potency testing of whole-cell Bordetella pertussis vaccines [3I]. The results of a nitric oxide production assay correlated with the results of the intracerebral mouse protection test that is currently used for these vaccines [32]. Furthermore, nitric oxide production was shown to be biologically relevant by protecting mice from severe infection during Bordetella pertussis infection in vivo $[33,34]$.

Taken together, the nitric oxide production assay with HDII cells may contribute to in vitro potency testing of inactivated vaccines against Av. paragallinarum. Future studies need to address whether there is a correlation between the results of this in vitro assay and the currently used in vivo vaccination-challenge test. Future studies may also address whether the nitric oxide production assay can be useful for potency testing of other inactivated bacterial poultry vaccines.

\section{Alternatives for the use of the HDI I cell line in cell-based assessment of inactivated poultry vaccines}

Chicken macrophage-like cell line MQ-NCSU

In addition to the HDII cell line, the chicken macrophage-like cell line MQ-NCSU [35] may be a promising alternative for use in cell-based potency assays. The cells show similar functionality compared to HDII cells including Fc receptor-mediated phagocytosis of opsonized antigens [35], and nitric oxide production and cytokine expression in response to bacteria [36,37]. The MQ-NCSU and HDII cell lines differ in background (normal vs. specific-pathogen free; both are derived from White Leghorn hens), tissue of origin (spleen vs. bone marrow) and viruses used for immortalization (myelocytomatosis virus vs. Marek's disease virus) [7,35]. We have conducted a pilot experiment in which nitric oxide production and phagocytosis were compared between MQ-NCSU and HDII cells in response to LPS and IBV antigen. We observed lower nitric oxide production by MQNCSU cells in response to LPS as compared to the HDI I cells, while nitric oxide was not produced in response to IBV antigen by any of the cell lines (not shown). Furthermore, we observed that phagocytosis was similarly enhanced in both cell lines in response to LPS or IBV antigen (not shown). Due to our observation that nitric oxide production was higher for the HDI I cell line we decided to focus on the HDI I cell line for use in cell-based assays to assess the immunostimulatory capacity of poultry vaccines. 
HEK reporter cells

Although the chicken macrophage-like cell line HDI I is able to show the immunostimulatory properties of inactivated poultry vaccines, the contribution of specific PRRs cannot be determined. It is not known whether inhibitors of mammalian PRRs can be used to block chicken PRRs, although we were able to use the LPS-binding antibiotic polymyxin to discriminate between LPS/TLR4-mediated and other responses. As described in Chapter 7, an alternative approach to determine the immunostimulatory properties of inactivated poultry vaccines would be to use reporter cells transfected with expression vectors encoding for chicken PRRs. Human embryonic kidney (HEK)-Blue reporter cell lines stably co-express human or murine TLRs and a nuclear factor (NF)- $\kappa \mathrm{B}$-inducible reporter gene to demonstrate TLR activation. Similar HEK reporter cell lines have successfully been created to demonstrate the functionality of chicken TLRs including TLRI/2 [38], TLR3[16] TLR4 [39], TLR5 [40], TLRI5 [4I] and TLR2I [42]. For chicken TLR7 HEK reporter cell lines were created, but led to conflicting results on TLR7 functionality in chickens $[16,17]$. The use of reporter cells expressing chicken TLRs in vaccine potency testing might be interesting to explore in future studies.

\section{The chicken bone marrow-derived dendritic cells as an in vitro tool in vaccine biomarker discovery}

\section{The potential of chicken bone marrow-derived dendritic cells in cell- based potency testing}

Immortalization of primary cells deemed suitable to create stable cell lines may result in high numbers of cells, but partial loss of functionality may be a consequence. It has been proposed to use dendritic cells (DCs) in cell-based assays for vaccine immunogenicity testing due to their important role in the initiation of the adaptive immune response [43]. For this purpose, we have characterized chicken bone marrow-derived DCs (chBMDCs) generated by culturing bone marrow cells of I8-days-old chicken embryos for one week in the presence of granulocyte/macrophage colony stimulating factor (GM-CSF) (Chapter 4). A chBMDC culture was described before $[44,45]$ and its heterogeneity has been recognized [44]. Nevertheless, the extent of this heterogeneity, including the potential presence of cell types other than DCs that may respond differently to stimuli $[46,47]$, had not been addressed before for the chBMDC culture.

We observed that multiple cell types were present in the chBMDC culture including two populations with DC-like characteristics, namely MHC-II low and MHC-Il high chBMDCs, which were different in morphology, phenotype and function. The MHC-I high chBMDCs were found to be more mature than their MHC-IIlow counterparts, both phenotypically and functionally. Furthermore, we observed that the mature phenotype of MHC-II high chBMDCs was reversible and that reseeding the mature cells after sorting led to a transition towards the relatively immature phenotype of the MHC-IIlow cells. Stimulation with LPS did not result in the full maturation of chBMDCs and instead led to an intermediate phenotype in which CD40 expression was increased, whereas CD80 and MHC-Il expression levels were slightly decreased. Due to the presence of different cell types including DCs at different states of maturation, we believe that the chBMDCs culture is less suitable for cell-based potency testing of vaccines than stable cell lines. 


\section{Proteomic analysis of vaccine-stimulated dendritic cells to gain better understanding about vaccine-induced immune responses}

Despite its limited potential in a cell-based assay for vaccine potency testing, the chBMDCs culture has moved research on chicken DCs forward and resulted in new reagents [48,49], as well as novel insights in DC responses to stimulation with immunostimulatory adjuvants [50-55], (pathogenic) microbes [56-60] and vaccines [5I, 56,6I-63]. Furthermore, the chBMDC culture has been used in studies using transcriptomic and proteomic analyses to investigate chicken DCs respond to adjuvants [55] and pathogens [60], respectively. In our study, we used chBMDCs to identify novel biomarkers of in vitro responses to inactivated poultry vaccines.

Proteomic analysis was conducted to investigate the responses of chBMDCs after stimulation with Escherichia coli LPS, inactivated IBV or an inactivated bivalent vaccine against IBV and NDV (Chapter 5). Similar to our studies with the HDII cells line, we found a stronger response to LPS than to inactivated IBV or the vaccine. After stimulation with LPS we observed a decreased expression of differentially expressed proteins (DEPs) associated with phagolysosomal activity, which may lead to better preservation of exogenous antigens for (cross-)presentation [64-67]. Furthermore, LPS stimulation led to an increase in DEPs associated with proteasomal antigen processing and antimicrobial activity. Two significantly upregulated DEPs of LPS-stimulated chBMDCs were also found as upregulated differentially expressed genes (DEGs) using RT-qPCR. These included GBP4L, involved in the activation of innate immune cells by LPS and bacteria [68], and ACSL, which has a role in the synthesis of the inflammatory mediator prostaglandin E2 [69]. It would be interesting to further explore GBP4L and ACSL as candidate biomarkers of bacterial poultry vaccines in future studies.

Since overlap between inactivated IBV antigen- and vaccine-stimulated chBMDCs was not observed, we hypothesize that the DEPs of vaccine-stimulated chBMDCs may be induced by the mineral oil adjuvant rather than by the antigen. We observed an increase of DEPs associated with lipid metabolism and storage. A previous study proposed that part of the activity of emulsion adjuvants may depend on its ability to change lipid metabolism in target cells, thereby affecting endolysosomal acidification and antigen presentation [70]. Two significantly upregulated DEPs of vaccine-stimulated chBMDCs included PLIN2, involved in the storage of lipid droplets, and PSMBI, a proteasomal subunit, which were also found as upregulated DEGs using RT-qPCR. Future studies are needed to determine whether these proteins can be used as vaccine-associated biomarkers in cell-based quality assessment of inactivated poultry vaccines. Whereas the chBMDC culture may be difficult to use in routine vaccine quality control due to its heterogeneity, our results demonstrate that the culture could be used to identify novel markers of the immunostimulatory capacity of poultry vaccines.

\section{T cell proliferation assay to assess cell-mediated immunity}

The previous part has described the activation of innate immune cells by inactivated poultry vaccines. For protective immunity to be induced by vaccines, antigen-presenting cells of the innate immune system need to activate antigen-specific $B$ and $T$ cells of the adaptive immune system. The activation of $B$ cells and subsequent antibody production is assessed 
in serological assays for potency assessment of vaccines. In contrast, assays to assess the activation of $T$ cells are not yet used for potency assessment of vaccines. In general, T cell assays cannot be used as animal-free alternative for vaccine potency testing, due to the need for blood collection from vaccinated animals to perform the assay, similar to currently used serological assays. However, animal-free $\mathrm{T}$ cell assays with $\mathrm{T}$ cell hybridoma cell lines might become promising in vitro alternatives in the future [7I]. As a first step, we investigated whether induction of cell-mediated immunity (CMI) in response to an inactivated poultry vaccine against IBV and NDV could be measured using an ex vivo $T$ cell proliferation assay (Chapter 6).

Peripheral blood mononuclear cells were collected from 33-weeks-old laying hens before and three weeks after vaccination with an inactivated poultry vaccine for IBV and NDV. These chickens were already exposed to a standard vaccination regiment including live attenuated vaccines for IBV and NDV before the onset of the study. In this way, the study reflected the field situation in which inactivated poultry vaccines are often used as booster vaccines and preceded by live attenuated vaccines. Upon ex vivo restimulation with inactivated IBV antigens, we observed proliferation of IBV-specific T cells when isolated before, but not after vaccination. In contrast, we did not observe $T$ cell proliferation after ex vivo restimulation with inactivated NDV antigens or an inactivated vaccine for both IBV and NDV, both before and after vaccination. In this study, we deed not observe an increase in antigen-specific $T$ cells after in vivo vaccination with the inactivated poultry vaccine for IBV and NDV. In contrast, some previous studies observed induction of $\mathrm{CMI}$ in chickens vaccinated with inactivated vaccines for IBV [72] and NDV [73,74], but to a lower level than found in chickens exposed to live attenuated vaccines [73,75] or virulent pathogens [76]. The $T$ cell proliferation assay in its current form was not sensitive enough for routine quality control of inactivated poultry vaccines for IBV and NDV. Future studies are required to improve the assay and to investigate whether $T$ cell responses correlate with protection after immunization with the inactivated vaccine for IBV and NDV.

\section{Advancements in in vitro potency testing of inactivated poultry vaccines}

The scientific constraints and potential solutions for replacement of in vivo batch tests of vaccines in general were discussed in a review (Chapter 7), many of which are applicable to inactivated poultry vaccines. Similar to many other vaccines, there is limited knowledge about the critical quality attributes of inactivated poultry vaccines. Vaccine properties other than antigen quantity or the capacity to induce humoral immunity have often been neglected. There is an increasing consensus that one-to-one replacement of in vivo tests may not always be achieved and a combination of in vitro alternatives to test for different vaccine properties may be needed. Other properties like the vaccine composition, susceptibility of the antigen to proteolytic degradation, the spatial organization of the antigen, and the presence of immunostimulatory molecules might require testing as well. Examples of the latter in inactivated poultry vaccines are allantoic fluid (Chapter 2) and Av. paragallinarum LPS (Chapter 3), which were not described before. In addition, there is still limited understanding about the mode-of-action of the mineral oil adjuvant used in many inactivated poultry vaccines. The function of this adjuvant has mostly been described as a depot that 
releases antigen over a long period of time [77,78]. Results reported in Chapter $\mathbf{5}$ suggest that the adjuvant of the inactivated poultry vaccines that were part of this study also interacts with immune cells and induces changes in cellular metabolism, proteasomal degradation and immune responses.

Another difficulty for the transition from in vivo to in vitro potency tested of poultry vaccines Chapter $\mathbf{7}$ is the incompatibility of adjuvants or other vaccine constituents with some in vitro potency tests (Chapter 7). In Chapter 3, we observed that purification of inactivated $A v$. paragallinarum bacteria from an adjuvanted poultry vaccine reduced the toxicity for the HDII cell line and led to increased nitric oxide production. The methods used to extract antigens from the adjuvanted vaccine, based on isopropyl myristate, were similar to those used for an enzyme-linked immunosorbent assay for NDV [79] and may be more widely applicable when adjuvants negatively affects the performance of in vitro assays. Of note, antigen extraction is undesirable when one also wants to determine the effects of adjuvants in cell-based assays.

\section{Concluding remarks}

The primary aim of this thesis was to set up in vitro cell-based assays to assess the immunostimulatory capacity of inactivated poultry vaccines and to determine their applicability for routine potency testing. Results of our investigations were clearly in favor of the use of cell-based potency assays for the inactivated Av. paragallinarum vaccine. Nitric oxide production and enhanced cytokine expression due to the presence of LPS was found. Hence, potency testing of this vaccine will most likely benefit by including a cell-based test to assess the immunostimulatory capacity of the vaccine. The applicability of cell-based assays for inactivated poultry vaccines of viral origin was less clear. A comparison between live attenuated and inactivated viral vaccines in future studies may indicate whether the inactivation process destroys the capacity of the viruses to stimulate innate immune cells. In the research presented in Chapter 2, we identified the immunostimulatory properties of allantoic fluid, a major constituent of inactivated poultry vaccines of viral origin, which led to enhanced Fc receptor-mediated phagocytosis through increased expression of CHIR$A B I$. In addition, we found (Chapter 5) that chBMDCs exposed to an inactivated poultry vaccines of viral origin showed many changes in the expression of proteins that are potentially interesting candidate biomarkers to use in a cell-based potency assays, including proteins like PLIN2 and PSMBI. The use of stable cell lines rather than chBMDCs is preferable to assess these biomarkers in future studies, since stable cell lines can be expanded into a large number of cells. Moreover, future studies are needed to further validate the nitric oxide production assay as a potency test for inactivated $A v$. paragallinarum vaccines, which may facilitate the replacement of currently used vaccination-challenge studies. Finally, the contribution of allantoic fluid to vaccine potency may be addressed in future studies. Eventually, multiple in vitro assays comprising physicochemical, immunochemical and cellbased assays may be required to substitute the different in vivo vaccine batch tests that are currently used for inactivated poultry vaccines. 


\section{References}

I. European Commission. Report on the Statistics on the Use of Animals for Scientific Purposes in the Member States of the European Union in 2015-2017.; 2019.

2. Milne C, Buchheit KH. EDQM's $3 R$ activities in the field of quality control of vaccines. ALTEX (20I2) I/I2.

3. Walker A, Srinivas GB. Opportunities and strategies to further reduce animal use for Leptospira vaccine potency testing. Biologicals (20I3) 4 I (5):332-337. doi:I0.10I6/j.biologicals.20I3.06.006

4. Schiffelers MJ, Blaauboer B, Bakker W, Hendriksen C. Replacing the NIH test for rabies vaccine potency testing: A synopsis of drivers and barriers. Biologicals (2014) 42(4):205-2 I7. doi: 10. I0 I6/j. biologicals.20I4.04.00 I

5. Uhlrich S, Coppens E, Moysan F, Nelson S, Nougarede N. 3Rs in quality control of human vaccines: Opportunities and barriers. In: Alternatives to animal testing. Springer Singapore; 2019:76-82. doi:10.1007/978-981-13-2447-5_10

6. The European Parliament and the Council of the European Union. DIRECTIVE 2010/63/EU on the protection of animals used for scientific purposes. Off J Eur Union (2010) 276:33-79.

7. Beug H, von Kirchbach A, Döderlein G, Conscience J-F, Graf T. Chicken hematopoietic cells transformed by seven strains of defective avian leukemia viruses display three distinct phenotypes of differentiation. Cell (1979) I8(2):375-390. doi:10.1016/0092-8674(79)90057-6

8. Liniger M, Summerfield A, Zimmer G, McCullough KC, Ruggli N. Chicken cells sense influenza A virus infection through MDA5 and CARDIF signaling involving LGP2. J Virol (20I2) 86(2):705717. doi:I0.1 I28/JVI.00742-II

9. Crippen TL. The selective inhibition of nitric oxide production in the avian macrophage cell line HDI I. Vet Immunol Immunopathol (2006) I09(I-2):I27-I37. doi:I0.10I6/j.vetimm.2005.08.024

10. Peroval MY, Boyd AC, Young JR, Smith AL, Muller M. A critical role for MAPK signalling pathways in the transcriptional regulation of toll like receptors. Gay N, ed. PLoS One (20I3) 8(2):e5 I 243. doi: 10.137I/journal.pone.005I243

II. Kato H, Takeuchi O, Sato S, Yoneyama M, Yamamoto M, Matsui K, et al. Differential roles of MDA5 and RIG-I helicases in the recognition of RNA viruses. Nature (2006) 44I (7089): I0 I- 105. doi: 10.1038/nature04734

12. Philbin VJ, lqbal M, Boyd Y, Goodchild MJ, Beal RK, Bumstead N, et al. Identification and characterization of a functional, alternatively spliced Toll-like receptor 7 (TLR7) and genomic disruption of TLR8 in chickens. Immunology (2005) II4(4):507-52I. doi:10.1III/j.13652567.2005.02125.x

13. Zhang P, Ding Z, Liu X, Chen Y, Li J, Tao Z, et al. Enhanced replication of virulent Newcastle disease virus in chicken macrophages is due to polarized activation of cells by inhibition of TLR7. Front Immunol (2018) 9(APR). doi:I0.3389/fimmu.20I8.00366

14. Herrera-Rodriguez J, Signorazzi A, Holtrop M, de Vries-Idema J, Huckriede A. Inactivated or damaged? Comparing the effect of inactivation methods on influenza virions to optimize vaccine production. Vaccine (2019) 37(12): I630-1637. doi:I0.1016/j.vaccine.2019.01.086

15. Hagenaars N, Mastrobattista E, Glansbeek H, Heldens J, van den Bosch H, Schijns V, et al. Headto-head comparison of four nonadjuvanted inactivated cell culture-derived influenza vaccines: Effect of composition, spatial organization and immunization route on the immunogenicity in a murine challenge model. Vaccine (2008) 26(5I):6555-6563. doi:10.1016/j.vaccine.2008.09.057

16. Schwarz H, Schneider K, Ohnemus A, Lavric M, Kothlow S, Bauer S, et al. Chicken toll-like receptor 3 recognizes its cognate ligand when ectopically expressed in human cells. J Interf Cytokine Res (2007) 27(2):97-I02. doi:10.1089/jir.2006.0098

17. Brownlie R, Zhu J, Allan B, Mutwiri GK, Babiuk LA, Potter A, et al. Chicken TLR2I acts as a functional homologue to mammalian TLR9 in the recognition of CPG oligodeoxynucleotides. Mol Immunol (2009) 46(I5):3163-3170. doi:10.1016/j.molimm.2009.06.002

18. Blackall PJ, Soriano E V. Infectious coryza and related bacterial infections. In: Saif YM, Fadly AM, Glisson JR, McDougald LR, Nolan LK, Swayne DE, eds. Diseases of Poultry. 12th ed. Blackwell 
Publishing; 2008:789-803.

19. Duff GW, Atkins E. The inhibitory effect of polymyxin B on endotoxin-induced endogenous pyrogen production. J Immunol Methods ( I 982) 52(3):333-340. doi: I0.1016/0022- I 759(82)900059

20. Jahromi MZ, Bello MB, Abdolmaleki M, Yeap SK, Hair-Bejo M, Omar AR. Differential activation of intraepithelial lymphocyte-natural killer cells in chickens infected with very virulent and vaccine strains of infectious bursal disease virus. Dev Comp Immunol (2018) 87:1 I6-I23. doi:10.1016/j. dci.2018.06.004

21. Duffy EB, Periasamy S, Hunt D, Drake JR, Harton JA. Fc $\gamma$ R mediates TLR2- and Syk-dependent NLRP3 inflammasome activation by inactivated Francisella tularensis LVS immune complexes. J Leukoc Biol (2016) I00(6): I335-I347. doi:I0.I I89/jlb.2A I I 5-555RR

22. Fang R, Du H, Lei G, Liu Y, Feng S, Ye C, et al. NLRP3 inflammasome plays an important role in caspase-I activation and IL-I $\beta$ secretion in macrophages infected with Pasteurella multocida. Vet Microbiol (2019) 231:207-213. doi:10.1016/j.vetmic.2019.03.019

23. Chung Y-H, Kim HY, Yoon BR, Kang YJ, Lee W-W. Suppression of Syk activation by resveratrol inhibits MSU crystal-induced inflammation in human monocytes. J Mol Med (2019) 97(3):369-383. doi:10.1007/s00109-018-01736-y

24. Bolin G, Burggren WW. Metanephric kidney development in the chicken embryo: Glomerular numbers, characteristics and perfusion. Comp Biochem Physiol - A Mol Integr Physiol (2013) I66(2):343-350. doi:I0.1016/j.cbpa.2013.07.0I I

25. Marrack P, McKee AS, Munks MW. Towards an understanding of the adjuvant action of aluminium. Nat Rev Immunol (2009) 9(4):287-293. doi: I0.1038/nri25I0

26. Martinon F, Pétrilli V, Mayor A, Tardivel A, Tschopp J. Gout-associated uric acid crystals activate the NALP3 inflammasome. Nature (2006) 440(708I):237-24I. doi:I0.1038/nature045I6

27. Lee C-C, Wu CC, Lin TL. Role of chicken melanoma differentiation-associated gene 5 in induction and activation of innate and adaptive immune responses to infectious bursal disease virus in cultured macrophages. Arch Virol (20I5) I60(I2):302I-3035. doi:I0.1007/s00705-0I5-26I2-y

28. Qi X, Liu C, Li R, Zhang H, Xu X, Wang J. Modulation of the innate immune-related genes expression in H9N2 avian influenza virus-infected chicken macrophage-like cells (HDII) in response to Escherichia coli LPS stimulation. Res Vet Sci (2017) III:36-42. doi:10.1016/j. rvsc.2016.11.008

29. García A, Romo F, Ortiz AM, Blackall PJ. The vaccination-challenge trial: the gold standard test to evaluate the protective efficacy of infectious coryza vaccines. Avian Pathol (2008) 37(2): I83-186. doi: 10.1080/0307945080192994 I

30. Maas PA, de Winter MPM, Venema S, Oei HL, Claassen IJTM. Antigen quantification as in vitro alternative for potency testing of inactivated viral poultry vaccines. Vet $Q 2000$. doi: $10.1080 / 01$ 652176.2000 .9695063

3I. Xing D, Markey K, Gaines Das R, Feavers I. Whole-cell pertussis vaccine potency assays: the Kendrick test and alternative assays. Expert Rev Vaccines (20 I4) I3(I0): I I 75-I I 82. doi: I0.1586 /I4760584.20I4.939636

32. Canthaboo C, Xing D, Corbel M. Development of a nitric oxide induction assay as a potential replacement for the intracerebral mouse protection test for potency assay of pertussis whole cell vaccines. Dev Biol Stand (1999) 101:95-103.

33. Elahi S, Van Kessel J, Kiros TG, Strom S, Hayakawa Y, Hyodo M, et al. c-di-GMP Enhances protective innate immunity in a murine model of pertussis. PLoS One (20/4) 9(I0):el09778. https://doi.org//0.137I/journal.pone.0109778.

34. Canthaboo C, Xing D, Wei XQ, Corbel MJ. Investigation of role of nitric oxide in protection from Bordetella pertussis respiratory challenge. Infect Immun (2002) 70(2):679-684. doi:I0.I I28/ IAI.70.2.679-684.2002

35. Qureshi MA, Miller L, Lillehoj HS, Ficken MD. Establishment and characterization of a chicken mononuclear cell line. Vet Immunol Immunopathol (1990) 26(3):237-250. doi:10.1016/01652427(90)90094-9 
36. Taha-Abdelaziz K, Astill J, Kulkarni RR, Read LR, Najarian A, Farber JM, et al. In vitro assessment of immunomodulatory and anti-Campylobacter activities of probiotic lactobacilli. Sci Rep (2019) 9(I): I7903. doi: 10.1038/s4I598-019-54494-3

37. Shojadoost B, Kulkarni RR, Brisbin JT, Quinteiro-Filho W, Alkie TN, Sharif S. Interactions between lactobacilli and chicken macrophages induce antiviral responses against avian influenza virus. Res Vet Sci (2019) I25:44I -450. doi:I0.I0I6/j.rvsc.20I7.I0.007

38. Higuchi M, Matsuo A, Shingai M, Shida K, Ishii A, Funami K, et al. Combinational recognition of bacterial lipoproteins and peptidoglycan by chicken Toll-like receptor 2 subfamily. Dev Comp Immunol (2008) 32(2):I47-I55. doi:I0.10 I6/j.dci.2007.05.003

39. Keestra AM, van Putten JPM. Unique Properties of the Chicken TLR4/MD-2 Complex: Selective Lipopolysaccharide Activation of the MyD88-Dependent Pathway. J Immunol (2008) I8I (6):43544362. doi: I0.4049/jimmunol. I8I.6.4354

40. Keestra AM, de Zoete MR, van Aubel RAMH, van Putten JPM. Functional characterization of chicken TLR5 reveals species-specific recognition of flagellin. Mol Immunol (2008) 45(5): 12981307. doi:10.1016/j.molimm.2007.09.013

4I. Boyd AC, Peroval MY, Hammond JA, Prickett MD, Young JR, Smith AL. TLRI5 Is Unique to Avian and Reptilian Lineages and Recognizes a Yeast-Derived Agonist. J Immunol (2012) I89(I0):49304938. doi: 10.4049/jimmunol. I I0I790

42. Keestra AM, de Zoete MR, Bouwman LI, van Putten JPM. Chicken TLR2I Is an Innate CPG DNA Receptor Distinct from Mammalian TLR9. J Immunol (20I0) 185(I):460-467. doi: 10.4049/ jimmunol.0901921

43. Vandebriel R, Hoefnagel MMN. Dendritic cell-based in vitro assays for vaccine immunogenicity. Hum Vaccin Immunother (20I2) 8(9): I323-1325. doi: I0.416I/hv.21350

44. Wu Z, Kaiser P. Antigen presenting cells in a non-mammalian model system, the chicken. Immunobiology (20II) 216(II):II77-I I83. doi:I0.1016/j.imbio.20II.05.0I2

45. de Geus ED, Tefsen B, van Haarlem DA, van Eden W, van Die I, Vervelde L. Glycans from avian influenza virus are recognized by chicken dendritic cells and are targets for the humoral immune response in chicken. Mol Immunol (2013) 56(4):452-462. doi:I0.1016/j.molimm.2013.06.007

46. Inaba K, Inaba M, Romani N, Aya H, Deguchi M, Ikehara S, et al. Generation of large numbers of dendritic cells from mouse bone marrow cultures supplemented with granulocyte/macrophage colony-stimulating factor. J Exp Med (1992) I76(6): I693-I702. doi:I0.1084/JEM.176.6.1693

47. Helft J, Böttcher J, Chakravarty P, Zelenay S, Huotari J, Schraml BU, et al. GM-CSF Mouse Bone Marrow Cultures Comprise a Heterogeneous Population of CDI Ic+MHCII+ Macrophages and Dendritic Cells. Immunity (20I5) 42(6): I I97-I2II. doi:I0.1016/j.immuni.20I5.05.0I8

48. Wu Z, Hu T, Butter C, Kaiser P. Cloning and characterisation of the chicken orthologue of dendritic cell-lysosomal associated membrane protein (DC-LAMP). Dev Comp Immunol (2010) 34(2): I83-I88. doi:I0.1016/J.DCl.2009.09.007

49. Wu Z, Hu T, Kaiser P. Chicken CCR6 and CCR7 are markers for immature and mature dendritic cells respectively. Dev Comp Immunol (20II) 35(5):563-567. doi:I0.10I6/J.DCI.20I0.12.0I5

50. Liang J, Fu J, Kang H, Lin J, Yu Q, Yang Q. The stimulatory effect of TLRs ligands on maturation of chicken bone marrow-derived dendritic cells. Vet Immunol Immunopathol (20I3) I55(3):205210. doi:I0.1016/J.VETIMM.2013.06.0I4

5I. Lin J, Yin YY, Qin T, Zhu LQ, Yu QH, Yang Q. Enhanced immune response of BMDCs pulsed with H9N2 AIV and CpG. Vaccine (2014) 32(50):6783-6790. doi:I0.1016/j.vaccine.20I4.10.0I3

52. Fu J, Liang J, Kang H, Lin J, Yu Q, Yang Q. The stimulatory effect of different CpG oligonucleotides on the maturation of chicken bone marrow-derived dendritic cells. Poult Sci (2014) 93(I):63-69. doi: I0.3382/ps.2013-0343।

53. Lin J, Kang H, Liang J, Fu J, Yu Q, Yang Q. CpG oligonucleotides and Astragalus polysaccharides are effective adjuvants in cultures of avian bone-marrow-derived dendritic cells. Br Poult Sci (2015) 56(I):30-38. doi:10.1080/0007I668.20I4.98II46

54. Larsen FT, Guldbrandtsen B, Christensen D, Pitcovski J, Kjærup RB, Dalgaard TS. Pustulan activates chicken bone marrow-derived dendritic cells in vitro and promotes ex vivo CD4+ $T$ cell recall 
response to infectious bronchitis virus. Vaccines (2020) 8(2):226. doi: 10.3390/vaccines8020226

55. Lin SY, Yao BY, Hu CMJ, Chen HW. Induction of robust immune responses by CPG-ODNloaded hollow polymeric nanoparticles for antiviral and vaccine applications in chickens. Int J Nanomedicine (2020) I5:3303-33|8. doi:I0.2147/IJN.S24I 492

56. Liang J, Yin Y, Qin T, Yang Q. Chicken bone marrow-derived dendritic cells maturation in response to infectious bursal disease virus. Vet Immunol Immunopathol (2015) 164:5I-55. doi:10.1016/j.vetimm.2014.12.012

57. Yasmin AR, Yeap SK, Tan SW, Hair-Bejo M, Fakurazi S, Kaiser P, et al. In vitro characterization of chicken bone marrow-derived dendritic cells following infection with very virulent infectious bursal disease virus. Avian Pathol (20I5) 44(6):452-462. doi:I0.1080/03079457.20I5.1084997

58. Huang $X$, Ma S, Wang L, Zhou H, Jiang Y, Cui W, et al. Lactobacillus johnsonii-activated chicken bone marrow-derived dendritic cells exhibit maturation and increased expression of cytokines and chemokines in vitro. Cytokine (2020) 136:155269. doi:10.1016/j.cyto.2020.155269

59. Xiang B, Zhu W, Li Y, Gao P, Liang J, Liu D, et al. Immune responses of mature chicken bonemarrow-derived dendritic cells infected with Newcastle disease virus strains with differing pathogenicity. Arch Virol (2018) 163(6): I407-I4I7. doi:I0.1007/s00705-0I8-3745-6

60. Yasmin AR, Omar AR, Farhanah MI, Hiscox AJ, Yeap SK. Quantitative proteomics analysis revealed compromised chicken dendritic cells function at early stage of very virulent infectious bursal disease virus infection. Avian Dis (2019) 63(2):275. doi:I0.1637/II936-0724I8-Reg.I

61. Kamble NM, Jawale CV, Lee JH. Activation of chicken bone marrow-derived dendritic cells induced by a Salmonella enteritidis ghost vaccine candidate. Poult Sci (2016) 95(10):2274-2280. doi: $10.3382 / p s / p e w 158$

62. Kamble NM, Jawale CV, Lee JH. Interaction of a live attenuated Salmonella gallinarum vaccine candidate with chicken bone marrow-derived dendritic cells. Avian Pathol (2016) 45(2):235-243. doi:10.1080/03079457.2016.1144919

63. Mou C, Zhu L, Yang J, Xu W, Cheng $X$, Yang $\mathrm{Q}$. Immune responses induced by recombinant Bacillus subtilis expressing the hemagglutinin protein of H5NI in chickens. Sci Rep (20I6) 6(I): III. doi:10.1038/srep38403

64. Accapezzato D, Visco V, Francavilla V, Molette C, Donato T, Paroli M, et al. Chloroquine enhances human CD8+ T cell responses against soluble antigens in vivo. J Exp Med (2005) 202(6):8I7-828. doi:10.1084/jem.2005II06

65. Alloatti A, Kotsias F, Magalhaes JG, Amigorena S. Dendritic cell maturation and cross-presentation: timing matters! Immunol Rev (20I6) 272(I):97-108. doi:I0.1 I I I/imr.I2432

66. Lennon-Duménil AM, Bakker AH, Maehr R, Fiebiger E, Overkleeft HS, Rosemblatt M, et al. Analysis of protease activity in live antigen-presenting cells shows regulation of the phagosomal proteolytic contents during dendritic cell activation. J Exp Med (2002) 196(4):529-539. doi:10.1084/jem.20020327

67. Pauwels AM, Härtlova A, Peltier J, Driege Y, Baudelet G, Brodin P, et al. Spatiotemporal changes of the phagosomal proteome in dendritic cells in response to LPS stimulation. Mol Cell Proteomics (2019) I8(5):909-922. doi:10.1074/mcp.RAII9.001316

68. Kim BH, Shenoy AR, Kumar P, Das R, Tiwari S, MacMicking JD. A family of IFN- $\gamma$-inducible 65kD GTPases protects against bacterial infection. Science (20II) 332(6030):7 I7-72I. doi: I 0. I I 26/ science. 1201711

69. Kanter JE, Kramer F, Barnhart S, Averill MM, Vivekanandan-Giri A, Vickery T, et al. Diabetes promotes an inflammatory macrophage phenotype and atherosclerosis through acyl-CoA synthetase I. Proc Natl Acad Sci U S A (2012) 109(12):E7 I 5-E724. doi: I0. I073/pnas. I I I 1600 I09

70. Kalvodova L. Squalene-based oil-in-water emulsion adjuvants perturb metabolism of neutral lipids and enhance lipid droplet formation. Biochem Biophys Res Commun (2010) 393(3):350-355. doi:10.1016/j.bbrc.2009.12.062

7I. Yu SCT, Nag B. Application of murine T-T hybridoma cells to in vitro potency assay of human synthetic peptide vaccines. Vaccine (1996) I4(14):I3|3-I32I. doi:10.1016/S0264$410 \times(96) 00066-7$ 
72. Timms LM, Bracewell CD. Cell mediated and humoral immune response of chickens to inactivated oil-emulsion infectious bronchitis vaccine. Res Vet Sci (1983) 34(2):224-230. doi:10.1016/s00345288(I8)32264-I

73. Dalgaard TS, Norup LR, Rubbenstroth D, Wattrang E, Juul-Madsen HR. Flow cytometric assessment of antigen-specific proliferation in peripheral chicken $T$ cells by CFSE dilution. Vet Immunol Immunopathol (2010) I38(I-2):85-94. doi:I0.1016/j.vetimm.2010.07.010

74. Norup LR, Dalgaard TS, Pedersen AR, Juul-Madsen HR. Assessment of Newcastle disease-specific T cell proliferation in different inbred MHC chicken lines. Scand J Immunol (20II) 74(I):23-30. doi:I0.1 III/j.1365-3083.20II.02534.x

75. Dalgaard TS, Norup LR, Pedersen AR, Handberg KJ, Jørgensen PH, Juul-Madsen HR. Flow cytometric assessment of chicken T cell-mediated immune responses after Newcastle disease virus vaccination and challenge. Vaccine (20I0) 28(28):4506-45I4. doi:I0.10I6/j.vaccine.20I0.04.044

76. Timms LM, Bracewell CD, Alexander DJ. Cell mediated and humoral immune response in chickens infected with avian infectious bronchitis. Br Vet J (1980) 136(4):349-356. doi: 10.1016/ S0007-1935(17)32237-6

77. Stils HF. Adjuvants and Antibody Production: Dispelling the myths associated with Freund's complete and other Adjuvants. ILAR J (2005) 46(3):280-293. doi:I0.1093/ilar.46.3.280

78. Herbert WJ. The mode of action of mineral-oil emulsion adjuvants on antibody production in mice. Immunology (1968) I4(3):30I-3I8.

79. Claassen I, Maas R, Oei H, Daas A, Milne C. Validation study to evaluate the reproducibility of a candidate in vitro potency assay of newcastle disease vaccines and to establish the suitability of a candidate biological reference preparation. Pharmeuropa bio (2004) 2004(I): I-I5. 



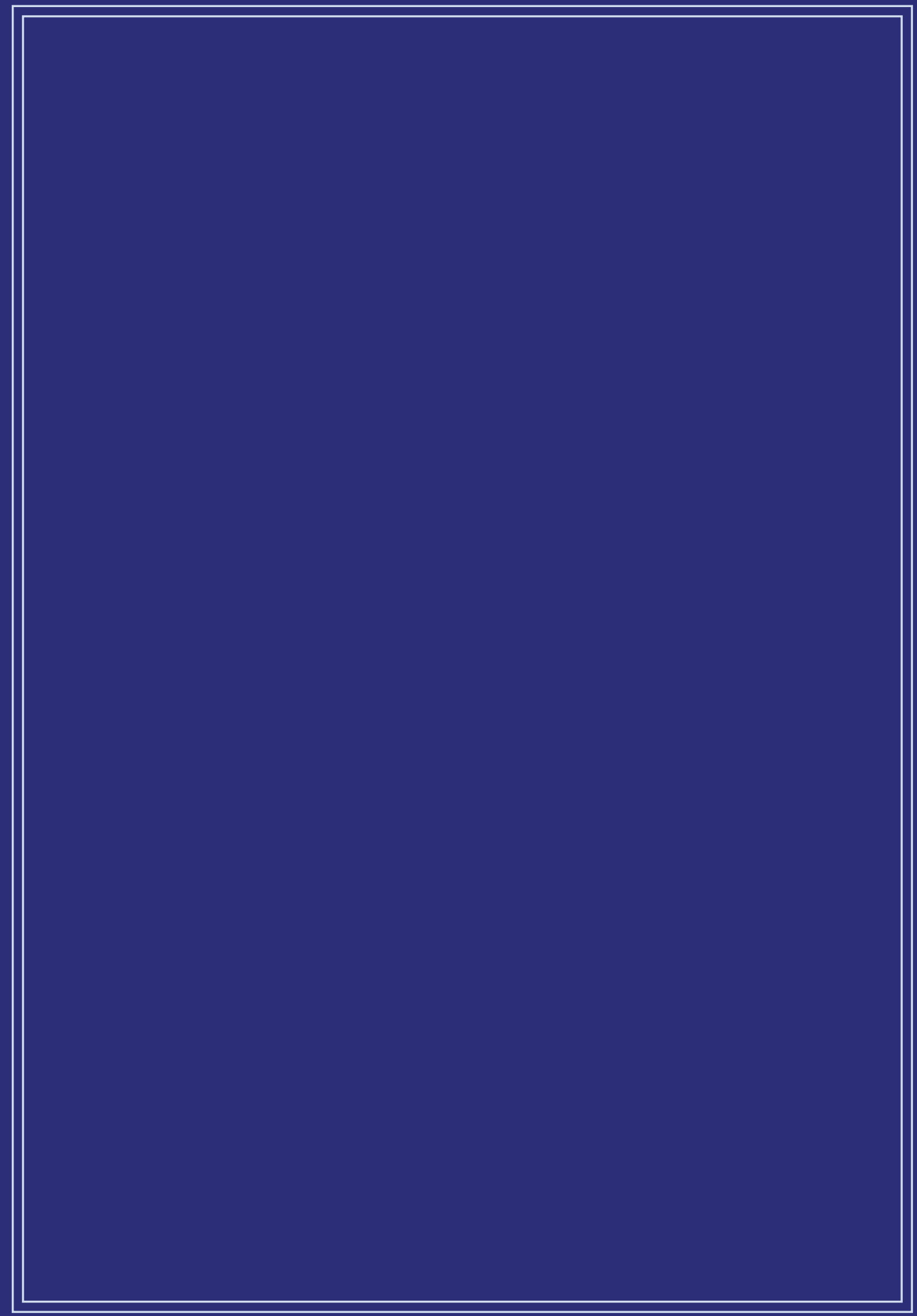


Appendix 


\section{Nederlandse samenvatting}

\section{Vaccinatie in pluimvee}

Kippen worden al vroeg in hun leven gevaccineerd met levende maar sterk verzwakte virussen en bacteriën. Het afweersysteem herkent de ziekteverwekkers en ontwikkelt een afweerreactie waarbij ook geheugencellen gevormd worden. Deze geheugencellen zorgen ervoor dat het afweersysteem sneller kan reageren op een toekomstige infectie met dezelfde ziekteverwekkers. Het aantal geheugencellen dat een ziekteverwekker herkent kan in de loop van de tijd afnemen. Vandaar dat leghennen en broedkippen, die relatief lang leven, op latere leeftijd opnieuw gevaccineerd worden. Ditmaal worden echter vaker vaccins gebruikt bestaande uit geïnactiveerde (gedode) ziekteverwekkers. Dit leidt tot hernieuwde en vaak sterkere reactiviteit en dus nog betere bescherming.

\section{De werking van het afweersysteem tijdens infectie of vaccinatie}

Het afweersysteem is grofweg in te delen in een aangeboren en een aangeleerd afweersysteem. Het aangeboren afweersysteem bestaat uit lichaamsbarrières (bijvoorbeeld slijmvliezen), (witte bloed)cellen en moleculen die moeten voorkomen dat ziekteverwekkers het lichaam binnendringen en zich daarin vermeerderen. De onderdelen van het aangeboren afweersysteem zijn bij de geboorte al aanwezig en zorgen voor onmiddellijke bescherming tegen ziekteverwekkers. Wanneer vaccins worden toegediend zullen de cellen van het aangeboren afweersysteem als eerste reageren en ziekteverwekkers onschadelijk te maken. Een belangrijk onderdeel van het aangeboren afweersysteem zijn antigeen-presenterende cellen: witte bloedcellen die ziekteverwekkers opnemen en afbreken tot kleine fragmenten, genaamd antigenen. Deze antigenen worden vervolgens gepresenteerd aan de cellen van het aangeleerde afweersysteem samen met instructies voor het opwekken van een afweerreactie die de ziekteverwekker efficiënt kan bestrijden.

Het aangeleerde afweersysteem omvat twee typen witte bloedcellen, B- en T-cellen, die de antigenen van ziekteverwekkers specifiek herkennen en zich vervolgens gaan vermenigvuldigen. De B-cellen produceren antistoffen die aan ziekteverwekkers in lichaamsvloeistoffen kunnen binden om ze te neutraliseren. De T-cellen zorgen voor het opruimen van geïnfecteerde lichaamseigen cellen. Nadat de ziekteverwekkers zijn opgeruimd daalt in het lichaam het aantal B- en T-cellen dat de ziekteverwekker specifiek herkent. Een deel van de antigeen-specifieke B- en T-cellen die tijdens de vermenigvuldiging ontstaan, bestaande uit de eerder genoemde geheugencellen, blijft echter aanwezig als voorbereiding op een eventuele nieuwe infectie. Ook vaccins zorgen voor het ontstaan van beschermende $B$ - en T-geheugencellen door op een veilige manier een infectie na bootsen.

\section{Proefdiergebruik bij het testen van vaccins}

Dierexperimenten zijn cruciaal voor het bepalen van de veiligheid en werkzaamheid tijdens de ontwikkelingsfase van vaccins. Ook na goedkeuring van vaccins blijven dierexperimenten vaak onderdeel van de kwaliteitscontrole die toegepast wordt op iedere nieuwe partij van geproduceerde vaccins. Proefdiergebruik voor kwaliteitscontrole van vaccins heeft een relatief groot aandeel in het proefdiergebruik in zowel Nederland als andere landen. Op allerlei gebieden zijn er ontwikkelingen gaande om het aantal proefdieren in experimenten te verminderen, ter vermindering van ernstig ongerief tijdens noodzakelijke dierexperimenten en om zoveel mogelijk dierexperimenten te vervangen door proefdiervrije alternatieven. 
Echter, voor de kwaliteitscontrole van kippenvaccins worden nog steeds veel proefdieren gebruikt. Er zijn met name nog geen proefdiervrije alternatieven voor het bepalen van de werkzaamheid van geïnactiveerde pluimveevaccins.

\section{Het doel van dit proefschrift}

$\mathrm{Er}$ is behoefte aan alternatieve methoden voor kwaliteitscontroles van geïnactiveerde pluimveevaccins gezien het nog steeds hoge proefdiergebruik bij deze toepassing. $\mathrm{Er}$ zijn verschillende alternatieven mogelijk, waaronder het bepalen van de hoeveelheid ziekteverwekker in een vaccin of tests met celculturen in plaats van proefdieren. Dit proefschrift beschrijft studies naar tests met gekweekte afweercellen die kunnen bijdragen aan het bepalen van de werkzaamheid van geïnactiveerde pluimveevaccins.

\section{Ontwikkeling van kwaliteitstests gebaseerd op cellijnen}

In de studies van hoofdstukken $\mathbf{2}$ en $\mathbf{3}$ worden tests beschreven die gebaseerd zijn op celculturen met een onsterfelijke cellijn van macrofagen, genaamd de HDII cellijn. Macrofagen zijn een type antigeen-presenterende cellen die onderdeel uitmaken van het aangeboren immuunsysteem. Wij hebben verschillende afweerfuncties van macrofagen gemeten. Eén van deze functies is de productie van stikstofmonoxide dat kan bijdragen aan het doden van ziekteverwekkers. Daarnaast is de opname van deeltjes door HDII cellen gemeten. Dit proces wordt fagocytose genoemd en is belangrijk voor het onschadelijk maken en doden van ziekteverwekkers. Ten slotte kunnen de HDII cellen na interactie met de ziekteverwekkers zogenoemde cytokinen produceren. Dit zijn moleculen die andere (afweer)cellen in het lichaam activeren, waaronder de cellen van het aangeleerde afweersysteem.

De studies beschreven in hoofdstukken $\mathbf{2}$ en $\mathbf{3}$ geven weer hoe HDII cellen reageren na stimulatie met verschillende geïnactiveerde (gedode) kippenvaccins bestaande uit gedode virussen en bacteriën. Deze vaccins beschermen kippen tegen de ziekteverwekkers van infectieuze bronchitis, de ziekte van Newcastle, 'egg drop syndrome' en infectieuze coryza, of combinaties van deze ziekten. In hoofdstuk $\mathbf{2}$ wordt beschreven dat één van de ingrediënten van kippenvaccins de opname van ziekteverwekkers (fagocytose) door HDI I cellen stimuleert. In hoofdstuk $\mathbf{3}$ wordt beschreven dat kippenvaccins met geïnactiveerde bacteriën de productie van stikstofmonoxide en cytokinen stimuleren. De mate waarin de HDII cellen geactiveerd worden en deze functies laten zien is indicatief voor de werkzaamheid van de verschillende vaccins. De beschreven methoden op basis van de HDI I cellijn zijn in staat om kippenvaccins op werkzaamheid te testen en kunnen daarmee bijdragen aan het vervangen van de huidige dierproeven.

\section{Biologische markers die activatie van het afweersysteem aantonen na stimulatie met vaccins}

Naast de doelgerichte aanpak die hierboven beschreven is hebben we ook gezocht naar nieuwe biologische markers die indicatief kunnen zijn voor de werkzaamheid van geïnactiveerde kippenvaccins. Naast macrofagen, waaronder de HDII cellijn, zijn ook dendritische cellen belangrijke antigeen-presterende cellen. Er zijn geen cellijnen die dendritische cellen van kippen representeren, maar in hoofdstuk $\mathbf{4}$ beschrijven wij een methode om dendritische cellen te kweken vanuit beenmerg afkomstig van kippenembryo's. De studie beschreven in hoofdstuk 5 geeft weer welke veranderingen dendritische cellen ondergaan ten gevolge 
van stimulatie met geïnactiveerde kippenvaccins. Op basis van deze studie hebben we verschillende veranderingen in de aanmaak en aanwezigheid van eiwitten geïdentificeerd die kunnen dienen als biologische markers voor de werkzaamheid van de vaccins.

\section{De rol van $\mathrm{T}$ cellen in de afweerreactie na vaccinatie met geïnactiveerde kippenvaccins}

Er zijn verschillende soorten dierproeven die gebruikt kunnen worden als kwaliteitstest voor de werkzaamheid van vaccins. Eén methode is het vaccineren en vervolgens infecteren van dieren, waarna gekeken wordt hoeveel dieren klachtenvrij blijven. Een veelgebruikte diervriendelijkere methode is het bepalen van de hoeveelheid antistoffen die het dier aanmaakt na vaccinatie. Zoals beschreven is het doel van vaccinatie ook om te zorgen dat het afweersysteem T-geheugencellen aanmaakt. Het is nog vrijwel onbekend of T-geheugencellen ook aangemaakt worden na vaccinatie met geïnactiveerde kippenvaccins en dit hebben we daarom onderzocht in de studie beschreven in hoofdstuk 6. Kippen werden gevaccineerd met een geïnactiveerd kippenvaccin voor bescherming tegen het infectieuze bronchitis virus (IBV) en een virus dat de ziekte van Newcastle veroorzaakt (NDV). Vervolgens zijn voor en na vaccinatie bloedmonsters genomen en zijn de witte bloedcellen opnieuw bloot gesteld aan de geïnactiveerde virussen om te bepalen of antigeen-specifieke T-geheugencellen aanwezig waren. Vooraf aan de vaccinatie vonden we celdeling van $\mathrm{T}$-cellen na herhaalde blootstelling met IBV, wat duidde op de aanwezigheid van $\mathrm{T}$-geheugencellen. $\mathrm{Na}$ de vaccinatie werd de celdeling van deze T-geheugencellen echter niet langer geobserveerd. Er kon dus niet worden vastgesteld dat de vaccinatie heeft geleid tot de aanmaak van antigeen-specifieke T-geheugencellen. De aanwezigheid van IBV-specifieke T-geheugencellen die we konden meten vooraf aan de vaccinatie was waarschijnlijk al eerder opgewekt door vaccinatie met een levend verzwakt vaccin tegen dit virus.

\section{Recente ontwikkelingen in de overgang naar proefdiervrij testen van vaccins}

De studies beschreven in dit proefschrift waren voornamelijk gericht op het ontwikkelen van proefdiervrije alternatieven voor het bepalen van de werkzaamheid van geïnactiveerde kippenvaccins. Hoofdstuk 7 beschrijft recente ontwikkelingen in het proefdiervrij testen van vaccins voor zowel dieren als mensen. Proefdiergebruik is voornamelijk omvangrijk bij het testen van geïnactiveerde vaccins en vaccins op basis van onschadelijk gemaakte gifstoffen van bacteriën (toxoïden). Voor sommige van deze vaccins zijn wel proefdiervrije alternatieven ontwikkeld om de werkzaamheid te bepalen. De meeste proefdiervrije tests zijn gebaseerd op het bepalen van de hoeveelheid geïnactiveerde ziekteverwekker in het vaccin. Het is echter duidelijk geworden dat dit niet altijd voldoende is om de werkzaamheid van vaccins te garanderen. Andere belangrijke eigenschappen zijn de samenstelling van het vaccin, structuurbehoud van de geïnactiveerde ziekteverwekker en de capaciteit om antigeen-presenterende cellen te activeren. Het is duidelijk dat meestal meer dan één proefdiervrije test nodig zal zijn om de huidige dierproeven te vervangen.

\section{Conclusies en blik op de toekomst}

Het doel van de studies beschreven in dit proefschrift was te onderzoeken of tests op basis van celculturen gebruikt kunnen worden om de werkzaamheid van geïnactiveerde kippenvaccins aan te tonen. In de studies van hoofdstukken $\mathbf{2}$ en $\mathbf{3}$ wordt aangetoond dat dergelijke tests inderdaad kunnen bijdragen aan het bepalen van de werkzaamheid. Daarnaast kunnen 
op basis van de biologische markers gevonden in de studie van hoofdstuk $\mathbf{5}$ nieuwe tests gebaseerd op celculturen ontwikkeld worden. Óf deze proefdiervrije tests daadwerkelijk dierexperimenten kunnen vervangen zal moeten blijken uit toekomstige studies waarin de proefdiervrije tests en dierexperimenten met elkaar vergeleken worden. Naar verwachting zal een combinatie van meerdere proefdiervrije tests nodig zijn om de huidige dierproeven voor geïnactiveerde kippenvaccins te kunnen vervangen. De tests die in dit proefschrift beschreven zijn kunnen hier een belangrijke bijdrage aan leveren. 


\section{Dankwoord /Acknowledgements}

Promoveren doe je niet alleen en ik wil hier dan ook graag de mensen bedanken die hebben bijgedragen aan de totstandkoming van dit proefschrift.

Christine, allereerst wil ik jou bedanken voor je uitgebreide begeleiding tijdens de afgelopen jaren. Ondanks dat we natuurlijk gebonden waren aan de afspraken van het VAC2VAC project, heb je mij de vrijheid gegeven om zelf ideeën voor onderzoeksprojecten aan te dragen en deze uit te voeren. Het was fijn dat je altijd erg toegankelijk en betrokken was, zowel voor werk-gerelateerde als persoonlijke dingen.

Victor, bedankt voor je betrokkenheid en kritische blik op alle hoofdstukken van dit proefschrift. Alhoewel de feedback soms overweldigend was heeft dit wel geleid tot een beter proefschrift en heb ik er veel van geleerd. Willem, bedankt voor je blijvende betrokkenheid bij mijn promotietraject, ook na je pensioen.

Everybody working at the Immunology division, thank you for sharing ideas, for all the nice conversations, and especially for all the fun at congresses, dissertation parties, holiday celebrations, lab days, and many more events.

First of all, I want to thank my former group members of the avian immunology group led by Christine, including Adil, Miriam, Daphne and Nathalie. Adil and Miriam, I am happy that you joined both the research group and office room W442 during the final year of my $\mathrm{PhD}$. Unfortunately, we were less able to spend time together due to the corona crisis, but it was a pleasure working with you. Daphne, we hebben heel wat tijd samen in het 'witte' lab doorgebracht. Ik had vooraf aan mijn PhD niet gedacht dat 'prakken' gezellig kan zijn. Bedankt dat ik altijd bij jou terecht kon voor hulp en gezelligheid. Nathalie, we hebben heel wat gekletst, gelachen en gefeest. We zijn naar Sardinië, Seattle en Oxford geweest, waar we genoten hebben van de voordelen van PhD'ers. Maar we hebben ook gezucht, gesteund en gevloekt. Dan was het fijn dat ik vaak een bondgenoot in je vond. In de afgelopen vier (bijna vijf) jaar ben je niet alleen een fijne collega, maar ook een goede vriendin geworden. Bedankt voor alles! Adil and Nathalie, thank you that you want to be my paranymphs.

The department changed a lot during my PhD project. I want to thank Anouk, Marit, Peter, New, Susan, Michael and Stefanie who made me feel welcome during the first years of my PhD. In het bijzonder wil ik mijn eerste kamergenootjes Peter, Manon en Charlotte bedanken. Bedankt dat ik altijd bij jullie terecht kon voor vragen of advies, maar vooral voor alle gezelligheid. Bart, Lena, Arie-Jan, Tabitha, Naomi, Emanuele, Lobna, and Daniëlle, I am glad that you came during the final two years of my PhD and created such a great work atmosphere. Bart, ik wil jou ook bedanken voor jouw hulp en advies bij de data-analyse van mijn proteomics experiment. Daniëlle, jij zorgt gegarandeerd voor gezelligheid onder alle omstandigheden, inclusief een pandemie door het coronavirus. Bedankt dat je hebt gezorgd voor een goede sfeer, ook nu dit alleen nog maar online mogelijk was. Andreja, the longer we worked together, the more I appreciated your sense of humor. Thank you for sharing ideas and thoughts about our projects, and for the nice company at many meetings, conferences and the coffee table. Qingkang, it was a pleasure working with you and I am looking forward to your defense in the near future. Coen, 
ondanks dat je bij het RIVM en het CBG werkte was je een gezellige extra collega die er vaak bij was tijdens VAC2VAC meetings, congressen en natuurlijk de cursus in Sardinië.

Op de afdeling Immunologie had ik de luxe dat een grote groep collega's er altijd voor zorgde dat alles op rolletjes liep. Peter, Aad, Irene, Daphne, Karin en Tara, bedankt dat jullie er altijd waren om het onderzoek op de afdeling mogelijk te maken door het wegwijs maken van medewerkers, te zorgen voor goede apparatuur en voorraden, en voor directe hulp bij het onderzoek. Ger, bedankt voor het beheren van de flow cytometers, je inzet voor de aanschaf van de CytoFLEX en voor je hulp bij de FACS experimenten. Alice, bedankt dat je onderdeel wilde uitmaken van mijn $\mathrm{PhD}$ begeleidingscommissie en voor je hulp bij het schrijven van verschillende manuscripten. Femke, bedankt voor je betrokkenheid en toegankelijkheid, ook als hoofd van de afdeling.

Richard en Esther, bedankt dat jullie altijd tijd hadden om mij te helpen met de fluorescentiemicroscopie en voor het meedenken over oplossingen voor vraagstukken in mijn onderzoek.

Ik wil graag de pluimveedierenartsen Mieke, Francisca en Marius, Andreas en de dierverzorgers, Maurits, Marc en Freek, bedanken voor het faciliteren van vaccinatie en bloedafname, en voor de verzorging van de dieren. Daarnaast wil ik Mieke bedanken voor haar deelname in mijn $\mathrm{PhD}$ begeleidingscommissie.

Hugo, Larissa en Jeroen, bedankt dat jullie mij hebben geholpen bij het proteomics experiment. Niet alleen hebben jullie het proteomics experiment mogelijk gemaakt, maar ik heb ook veel van jullie geleerd over massa spectometrie en de bijhorende data-analyse.

Arjen, Coenraad, Marcel, Rob en Anke, bedankt voor de leuke en interessante gesprekken van afgelopen tijd, en tijdens eerdere VAC2VAC meetings, die hebben geleid tot het review over proefdiervrije alternatieven voor het testen van vaccines.

Büsra, Laurens, Szymon and Sabine, thank you for your help in various side projects. I have really enjoyed supervising you during your internships.

Ik wil de leden van mijn beoordelingscommissie bedanken voor hun geïnvesteerde tijd om mijn proefschrift te lezen en te beoordelen.

All the people from the Veterinary PhD Council and the Infection and Immunity Programme, especially the organizers of many events, thank you for all the nice interactions we have had.

Mijn ouders, bedankt dat jullie mij al 27 jaar lang gestimuleerd hebben om alle kansen te benutten. Mijn middelbare school, de universiteit, mijn PhD, en nu mijn baan als postdoc, ik geniet er (bijna) iedere dag van en ik ben dankbaar voor alles waar jullie me mee geholpen hebben. En ook als het even wat minder gaat kan ik altijd bij jullie terecht. Kortom, bedankt!

Ook mijn zus Lisanne, mijn opa, de rest van mijn familie, mijn schoonfamilie en mijn vrienden wil ik bedanken. Bedankt voor jullie betrokkenheid en interesse. "Hoe gaat het 
met de kippen?" was een standaard vraag, met een knipoog, die ik vaak gehoord heb. Zie hier het resultaat!

Ten slotte, lieve Svenja, bedankt dat je er altijd voor mij bent. Als AIOS en AIO besteedden wij wel eens wat avonden of weekenden aan werk, maar gelukkig doen we samen ook veel leuke dingen om te ontspannen. We de afgelopen jaren een fijn leven opgebouwd met mooi huis in onze favoriete stad en waarin we inmiddels elkaars verloofden zijn. Dit jaar gaan we gezellig samen naar het Leiden voor nieuwe avonturen. Je stimuleert me, je steunt me, je bent een luisterend oor en je maakt me vooral heel erg blij. 


\section{Curriculum Vitae}

Robin Henricus Godefridus Antonius van den Biggelaar was born on July 5th, 1993 in Geldrop, the Netherlands. After graduating from the WereDi Gymnasium in Valkenswaard in 20II, he started his studies in Biomedical Sciences at Utrecht University. During this bachelor's program, he participated in an extra-curricular honors program. Furthermore, he conducted a short internship under supervision of dr. Helmi Pett and Prof. dr. Teun Bousema at the Department of Medical Microbiology at the Radboud University Medical Centre. Here, he also wrote his bachelor thesis about the use of primaquine against Plasmodium falciparum, the causative agent of malaria, in endemic countries where glucose 6-phosphate dehydrogenase (G6PD) deficiency is common. He obtained his bachelor's degree in 2014, after which he continued his studies with the master program Infection and Immunology at Utrecht University. Between 20I4-20I5, he conducted a 9-month internship at the Virology division of the Faculty of Veterinary Medicine at Utrecht University under supervision of dr. Mark Bakkers and dr. Raoul de Groot. Here, he participated in elucidating the importance of coronavirus hemagglutinin esterase in the dynamic interaction between the viral particle and host sialic acids during infection. Between 20I5-20I6, he did another 6-month internship at the Centre for Infectious Medicine of the Karolinska Institute, in Stockholm, Sweden under supervision of dr. Joana Dias, dr. Edwin Leeansyah and Prof. dr. Johan Sandberg. The research focused on elucidating the phenotypical and functional differences between CD4- CD8 ${ }^{+}$and CD4- CD8- mucosa-associated invariant T cells. Robin obtained his master's degree in 2016.

In 2016, Robin started his PhD project the Immunology division of the Faculty of Veterinary Medicine at Utrecht University under supervision of Prof. dr. Willem van Eden, Prof. dr. Victor Rutten and dr. Christine Jansen. His research concerned the development of in vitro alternatives to the quality tests that are currently conducted in vivo for batch release of inactivated poultry vaccines, which resulted in this thesis. During his $\mathrm{PhD}$ project, Robin was an active member of the Veterinary $\mathrm{PhD}$ council, organizing lectures and workshops for fellow PhD students. In 2021, Robin continued his scientific career as a postdoctoral researcher at Leiden University and the Leiden University Medical Centre under supervision of Prof. dr. Annemarie Meijer, Prof. dr. Tom Ottenhoff and dr. Marielle Haks to work on host-directed therapies for the treatment of Mycobacterium tuberculosis. 


\section{List of publications}

Dias J, van den Biggelaar RHGA, Boulouis C, Gorin J-B, Lal KG, Gibbs A, et al. The CD4-CD8- MAIT cell subpopulation is a functionally distinct subset developmentally related to the main CD8+ MAIT cell pool. Proc Natl Acad Sci U S A (20 I8) II5(49). doi:I0.I073/ pnas. $1812273 \mid 15$

van den Biggelaar RHGA, van Eden W, Rutten VPMG, Jansen CA. Nitric oxide production and $\mathrm{Fc}$ receptor-mediated phagocytosis as functional readouts of macrophage activity upon stimulation with inactivated poultry vaccines in vitro. Vaccines (2020) 8(2):332. doi: I0.3390/ vaccines 8020332

van den Biggelaar RHGA, van Eden W, Rutten VPMG, Jansen CA. Macrophage activation assays to evaluate the immunostimulatory capacity of Avibacterium paragallinarum in a multivalent poultry vaccine. Vaccines (2020) 8(4):67I. doi: I0.3390/vaccines804067I

van den Biggelaar RHGA, Arkesteijn GJA, Rutten VPMG, van Eden W, Jansen CA. In vitro chicken bone marrow-derived dendritic cells comprise subsets at different states of maturation. Front Immunol (2020) I I. doi: I0.3389/fimmu.2020.00 I4 I

Verwoolde MB, van den Biggelaar RHGA, de Vries Reilingh G, Arts JAJ, van Baal J, Lammers $A$, et al. Innate immune training and metabolic reprogramming in primary monocytes of broiler and laying hens. Dev Comp Immunol (2020) I I4: 1038 I I. doi: I 0. I0 I6/j. dci.2020.1038II

Verwoolde MB, van den Biggelaar RHGA, van Baal J, Jansen CA, Lammers A. Training of primary chicken monocytes results in enhanced pro-inflammatory responses. Vet Sci (2020) 7(3): I I5. doi: I0.3390/VETSCI7030I I5

Peng L, van den Biggelaar RHGA, Jansen CA, Haagsman HP, Veldhuizen EJA. A method to differentiate chicken monocytes into macrophages with proinflammatory properties. Immunobiology August 2020:I52004. doi:I0.1016/j.imbio.2020.152004

van den Biggelaar RHGA, van der Maas L, Meiring HG, Pennings JLA, van Eden W, Rutten VPMG, Jansen CA. Proteomic analysis of chicken bone marrow-derived dendritic cells in response to an inactivated IBV+NDV poultry vaccine. Submitted

van den Biggelaar RHGA, Hoefnagel MHN, Vandebriel RJ, Sloots A, Hendriksen CFM, van Eden W, Rutten VPMG, Jansen CA. Overcoming scientific barriers in the transition to from in vivo to non-animal batch testing of human and veterinary vaccines. Submitted

Meijerink N, van den Biggelaar RHGA, van Haarlem DA, Stegeman JA, Rutten VPMG, Jansen A. Contribution of innate and adaptive immune cells to the elimination of Salmonella enterica serotype Enteritidis infection in young broiler chickens. Submitted 